University of Tennessee Health Science Center UTHSC Digital Commons

\title{
Novel Paclitaxel Nanoparticles for Enhanced Therapeutic Effects in Breast Cancer
}

Pallabita Chowdhury

University of Tennessee Health Science Center

Follow this and additional works at: https://dc.uthsc.edu/dissertations

Part of the Pharmacy and Pharmaceutical Sciences Commons

\section{Recommended Citation}

Chowdhury, Pallabita (https://orcid.org/0000-0003-0561-3204), "Novel Paclitaxel Nanoparticles for Enhanced Therapeutic Effects in Breast Cancer" (2020). Theses and Dissertations (ETD). Paper 515. http://dx.doi.org/10.21007/etd.cghs.2020.0500. 


\title{
Novel Paclitaxel Nanoparticles for Enhanced Therapeutic Effects in Breast Cancer
}

\author{
Abstract \\ Drug delivery to triple negative breast cancer (TNBC) remains a formidable challenge. Given the lack of \\ expressions of estrogen (ER), progesterone (PR) and human epidermal growth factor receptor (HER2), \\ there is no specific prognostic marker for targeting this aggressive cancer. In spite of rapid advancement \\ in breast cancer with agents such as Trastuzumab in HER2 positive, chemotherapy with cytotoxic agents \\ remains the mainstay treatment for TNBC. Although these cytotoxic agents are potent in managing the \\ tumor progression, yet they pose adverse effects on healthy tissues. Paclitaxel (PTX) has been used as a \\ gold standard chemotherapeutic agent for breast, ovarian, pancreatic and non-small cell lung carcinoma. \\ Till date, PTX has been used as the first line of treatment for metastatic breast cancer. However, being \\ biopharmaceutical classification system (BCS) class IV agent PTX suffers low solubility, poor \\ permeability and remains a major challenge for efficient delivery specific to the tumor sites. Various \\ formulations of PTX have been developed and approved in some countries, however due to serious \\ adverse effects, suffer from poor patient outcomes.In this regard, we choose PTX as our model drug to \\ generate, a safe and efficacious nanoparticle formulation by nanoprecipitation technique. For this, we \\ screened commonly used pharmaceutical excipients and polymers and found the best candidate with \\ built-in chemosensitization motifs (tannic acid: TA) and polyvinyl/pyrrolidone (PVP), given their innate \\ potential to prevent drug efflux and capability to form the structured nanoparticle core due to extensive \\ hydrogen bonding. The optimized formulation of TA and PVP nanoparticles had an average diameter of \\ $102.22 \pm 14.05 \mathrm{~nm}$ and $140.53 \pm 7.08 \mathrm{~nm}$, respectively. A drug encapsulation efficiency of $\sim 96 \%$ was \\ evaluated by LC-MS/MS. Improved in vitro and in vivo efficacies were achieved with respect to plain drug, \\ attributed to the inhibitory P-gp effects of TA. To expand the scope of the developed formulation for \\ enhanced specificity to TNBC tumors, we screened endogenous cell membranes (Human neutrophil, \\ Mesenchymal s

\section{Document Type} \\ Dissertation

\section{Degree Name} \\ Doctor of Philosophy (PhD) \\ Program \\ Pharmaceutical Sciences \\ Research Advisor \\ Murali Mohan Yallapu,Ph.D.

\section{Keywords} \\ Biomimetic nanoparticles, Nanoparticles, Targeted drug delivery, Triple negative breast cancer

\section{Subject Categories} \\ Medicine and Health Sciences | Pharmacy and Pharmaceutical Sciences
}


UNIVERSITY OF TENNESSEE HEALTH SCIENCE CENTER

Doctor OF PhILOSOPHY DisSERTATION

\section{Novel Paclitaxel Nanoparticles for Enhanced Therapeutic Effects in Breast Cancer}

Author:

Pallabita Chowdhury
Advisor:

Murali Mohan Yallapu, PhD

A Dissertation Presented for The Graduate Studies Council of

The University of Tennessee Health Science Center

in Partial Fulfillment of the Requirements for the

Doctor of Philosophy degree from

The University of Tennessee

in

Pharmaceutical Sciences: Pharmaceutics

College of Graduate Health Sciences

May 2020 
Chapter 3 (c) 2018 Elsevier Inc.

All other material (C) 2020 by Pallabita Chowdhury. All rights reserved. 


\section{DEDICATION}

Dedicated to my loving family and friends like family in Memphis. 


\section{ACKNOWLEDGEMENTS}

I would like to express my heartfelt sincere gratitude to all the people who has been a constant source of support and inspiration throughout my journey at University of Tennessee Health Science Center (UTHSC). I may not be able to list all the names here, but I sincerely appreciate for all the unforgettable help, support, advice, and encouragement that I have received at UTHSC.

Firstly, I would like to express my sincerest gratitude to my advisor, Dr. Murali M. Yallapu, for his constant support, encouragement, and guidance throughout my graduate studies. I am truly thankful to him for the immense opportunities, motivation, and support that he provided. I am grateful for his constant support and guidance in planning, executing experiments, plan of research study, writing manuscripts, and preparing the dissertation. He has provided constant encouragement and support to help me build a successful career and I am very grateful to have the honor to work with him. I express my deepest gratitude to all the graduate committee members Dr.Subhash Chauhan, Dr. Santosh Kumar, Dr. Bernd Meibohm, Dr.Wei Li, and Dr. Ramesh Narayanan for their constant time, support, helpful suggestions, and insightful comments. I am grateful for the kindness, love and academic support they all offered during my graduate studies. I sincerely thank our collaborator, Dr. Subhash Chauhan, for his insightful expertise in cancer research. I would like to thank Dr. Bernd Meibohm for graciously allowing to use the lab facilities and seeking advice from his graduate student. I thank our collaborator, Dr. Santosh Kumar, for his help and support especially during the latter phase of my graduate career.

I would like to thank my lab and collaborator lab members: Dr. Prashanth Bhushetty, Elham Hatami, Saini Setua, Nirnoy Dan, Advait Shetty, Kamalika Samanta, and Mehdi Chaib for their help and support at various stages of the work. I would also like to thank Dr. T.J. Hollingsworth from Imaging Facility at UTHSC. Also, I am grateful to all the faculty members of the Department of Pharmaceutical Sciences especially Dr. Frank Park for graciously allowing us to use his lab facilities. I would like to thank our staff members Shelley Cannioto, Kendra Golden, Tambra Moten, Erica Jones, Lisa Schaffer, and ex-staff Mary Newell for their love and support. My heartfelt gratitude to UTHSC, College of Pharmacy, and College of Graduate Health Sciences for all the facilities, equipment, and financial support. I would like to thank all the funding agencies and UTHSC for Alma and Hall Reagan Endowment Fellowship

I would like to thank my friends like family in Memphis who has made this journey a memorable one. Their valuable source of friendship and advice over the past years has made me kept going in this roller coaster ride of graduate studies. Last but not the least, I am very much grateful to Santosh Wagh for being the backbone of my constant support. My mom, dad, sister and beloved nephew, this is the culmination of all of their love and effort, and without their love, I would not have been here. Finally, I thank God for giving me this opportunity for being where I am today. 


\begin{abstract}
Drug delivery to triple-negative breast cancer (TNBC) remains a formidable challenge. Given the lack of expressions of estrogen (ER), progesterone (PR) and human epidermal growth factor receptor (HER2), there is no specific prognostic marker for targeting this aggressive cancer. In spite of rapid advancement in breast cancer with agents such as Trastuzumab in HER2 positive, chemotherapy with cytotoxic agents remains the mainstay treatment for TNBC. Although these cytotoxic agents are potent in managing the tumor progression, yet they pose adverse effects on healthy tissues. Paclitaxel (PTX) has been used as a gold standard chemotherapeutic agent for breast, ovarian, pancreatic and non-small cell lung carcinoma. Till date, PTX has been used as the first line of treatment for metastatic breast cancer. However, being a biopharmaceutical classification system (BCS) class IV agent PTX suffers low solubility, poor permeability and remains a major challenge for efficient delivery specific to the tumor sites. Various formulations of PTX have been developed and approved in some countries, however, due to serious adverse effects, suffer from poor patient outcomes.

In this regard, we choose PTX as our model drug to generate, a safe and efficacious nanoparticle formulation by nanoprecipitation technique. For this, we screened commonly used pharmaceutical excipients and polymers and found the best candidate with built-in chemosensitization motifs (tannic acid: TA) and polyvinyl/pyrrolidone (PVP), given their innate potential to prevent drug efflux and capability to form the structured nanoparticle core due to extensive hydrogen bonding. The optimized formulation of TA and PVP nanoparticles had an average diameter of $102.22 \pm 14.05 \mathrm{~nm}$ and $140.53 \pm 7.08 \mathrm{~nm}$, respectively. A drug encapsulation efficiency of $\sim 96 \%$ was evaluated by LC-MS/MS. Improved in vitro and in vivo efficacies were achieved with respect to plain drug, attributed to the inhibitory P-gp effects of TA. To expand the scope of the developed formulation for enhanced specificity to TNBC tumors, we screened endogenous cell membranes (Human neutrophil, Mesenchymal stem cells, RAW 24 6.7). Here we present a final optimized biomimetic nanoformulation of PTX coated with activated human neutrophil membrane (PVT-NEU NPs). These nanoparticles have expression of proteins on cell membrane [Lymphocyte function-associated antigen1 (LFA-1), macrophage-1 antigen (Mac-1), platelet endothelial cell adhesion molecule-1 (PECAM-1), P-selectin glycoprotein ligand-1 (PSGL-1)] that have high affinity for receptors that are overexpressed on the surface of TNBC tumors [Intercellular adhesion molecule (ICAM-1/2), vascular cell adhesion protein-1 (VCAM-1), junctional adhesion molecules (JAMs), E-selectin, P-selectin]. Due to these specific receptor-ligand interactions, PVT-NEU NPs show improved in vitro and in vivo efficacies and reduced non-specific binding and off-target toxicities, in comparison to uncoated polymeric nanoparticles. These results suggest, potential of biomimetic nanoparticles as a promising drug delivery option for metastatic breast cancer.
\end{abstract}




\section{TABLE OF CONTENTS}

CHAPTER 1. INTRODUCTION ......................................................................................1

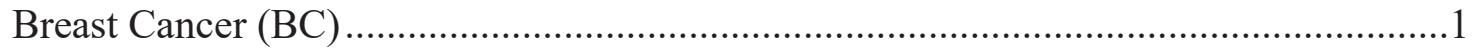

BC Anatomy Causes and Risks ................................................................... 1

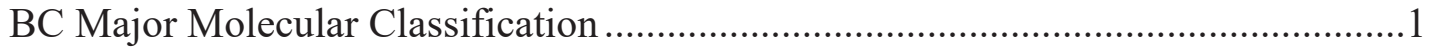

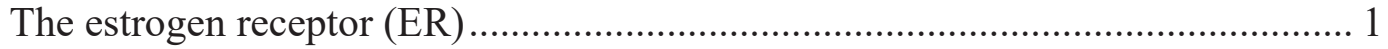

Human epidermal growth factor receptor 2 (HER2) ........................................ 4

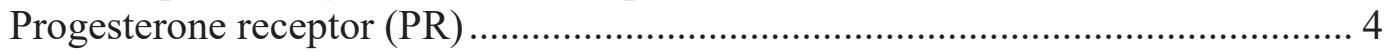

Triple-negative breast cancer (TNBC) ....................................................... 5

Therapeutic Strategies for TNBC, Evaluated by Clinical Trials ................................5

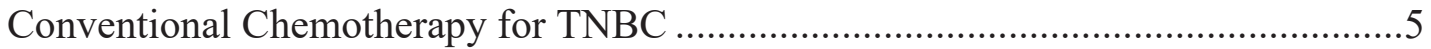

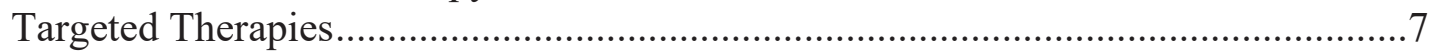

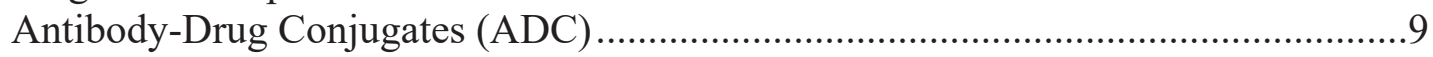

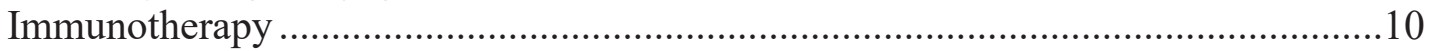

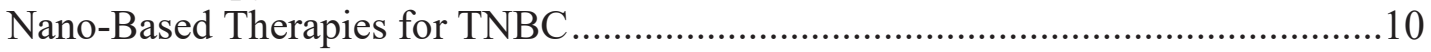

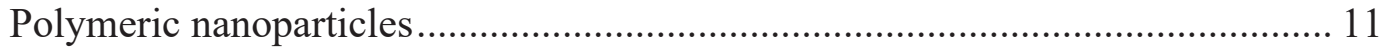

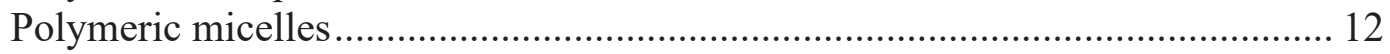

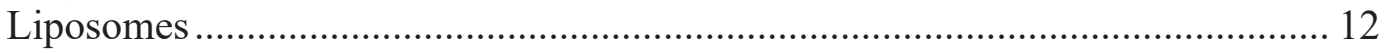

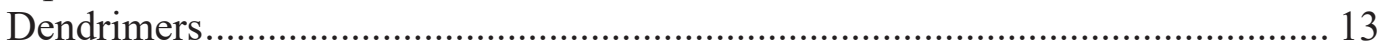

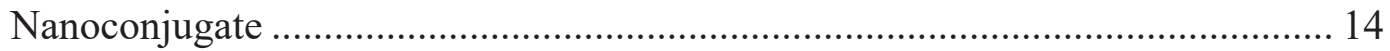

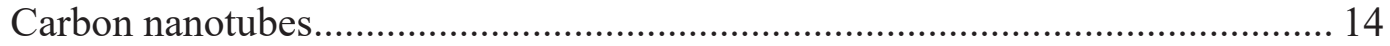

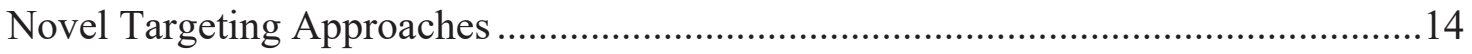

Hematopoietic Stem Cells as Carrier for Targeted Delivery ..................................15

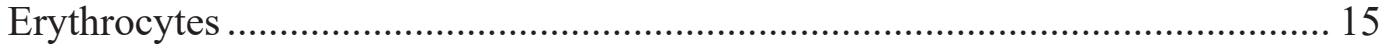

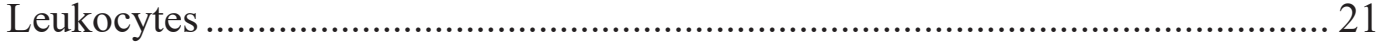

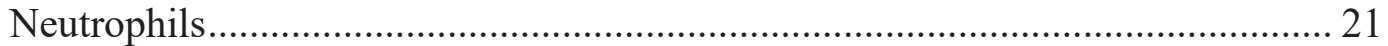

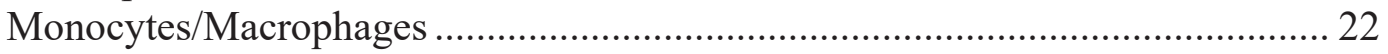

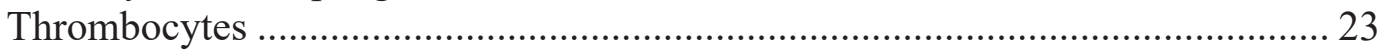

Cell-Membrane Coated Nanoparticles for Targeted Delivery ................................24

Erythrocyte membrane-coated nanoparticles................................................... 26

Neutrophil membrane-formed nanoparticles .................................................... 27

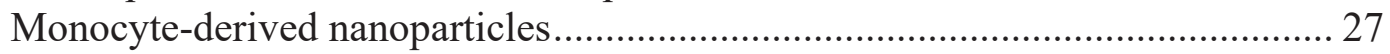

Platelet membrane-coated nanoparticles................................................... 28

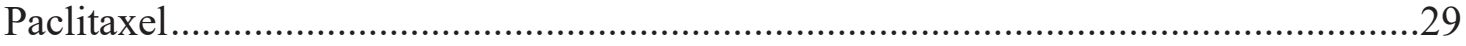

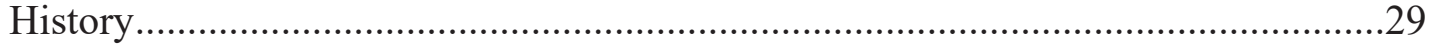

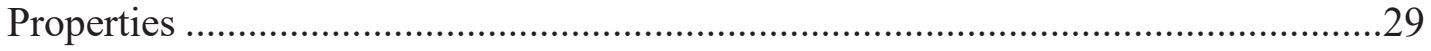

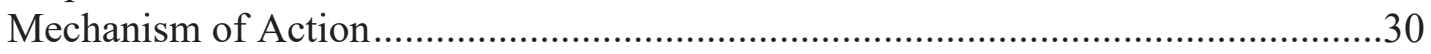

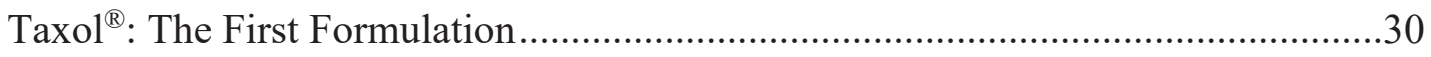

Nano-Based PTX Formulations for TNBC in Preclinical and Clinical Phase...........30

Albumin-based nanoparticles: Abraxane or nab-PTX …................................. 31

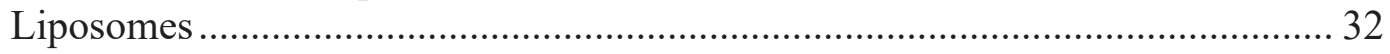

Polymeric micelles .............................................................................. 33

Polymeric-lipidic nanoparticle: PICN or PTX injection concentrate for

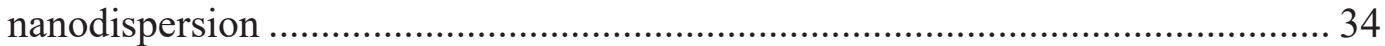




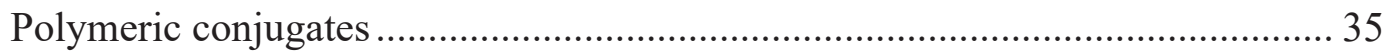

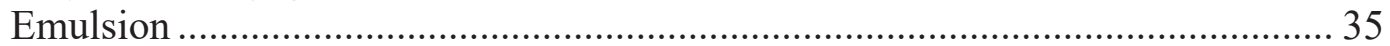

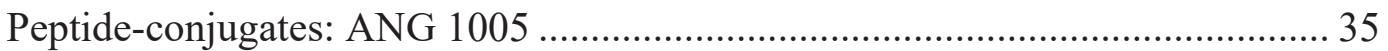

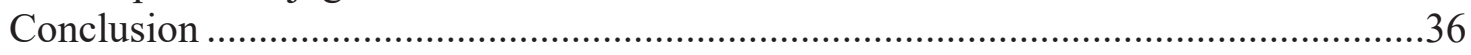

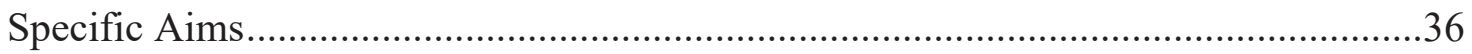

Aim 1: To Improve Paclitaxel-Mediated Delivery, by Developing and

Characterizing Polymer-Based Self-Assembled Paclitaxel Nanoparticles for BC ....37

Aim 2: To Develop and Characterize Nanoparticle-Based Paclitaxel Formulation to Enhance Therapeutic Benefits Using P-gp Efflux Inhibitor, such as Tannic

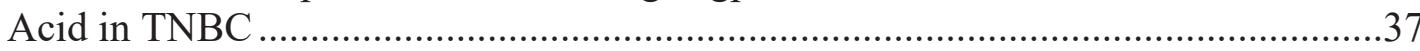

Aim 3: To Enhance Targetability of Paclitaxel in TNBC Tumors by Developing and Characterizing Biomimetic Nanoparticles Using Endogenous Cell

Membrane, such as Human Neutrophils

\section{CHAPTER 2. DEVELOPMENT OF POLYVINYL/PYRROLIDONE-PTX} SELF-ASSEMBLIES FOR BC.......................................................................................38

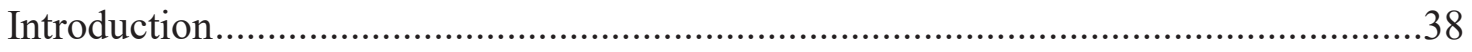

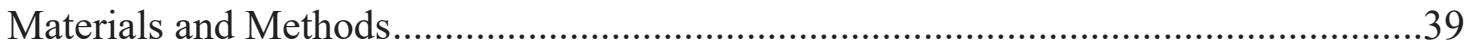

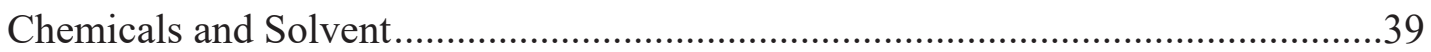

Cell Culture, Growth Conditions and Treatment ......................................................39

Preparation of Polymer-PTX-SAs (Poly/PTX-SAs) .................................................42

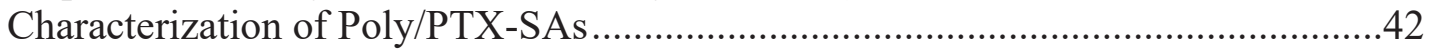

Particle size and zeta potential ........................................................................ 42

Transmission electron microscopy ………………................................................ 43

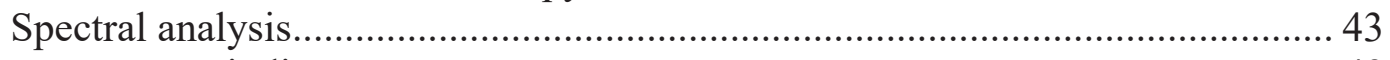

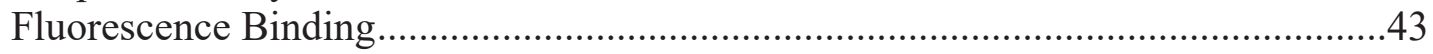

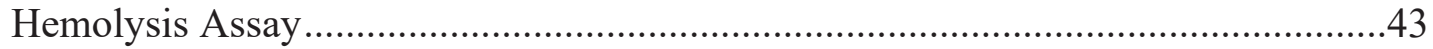

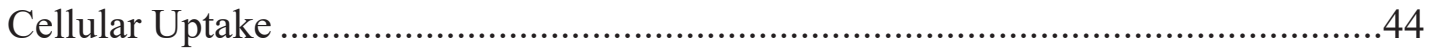

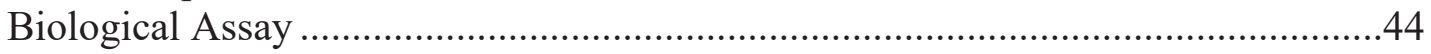

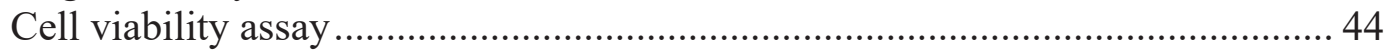

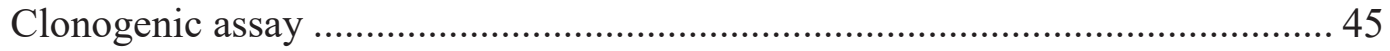

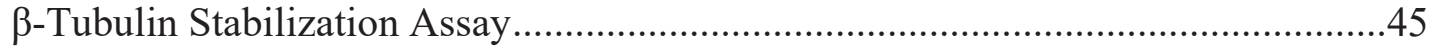

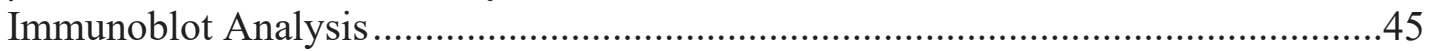

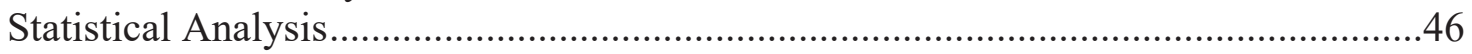

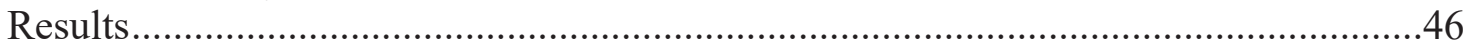

Development of a Suitable Poly/PTX-SA Formulation for BC ..................................46

Characterization of PVP/PTX SA Formation ......................................................49

In Vitro Anticancer Potential of PVP/PTX-SAs ....................................................52

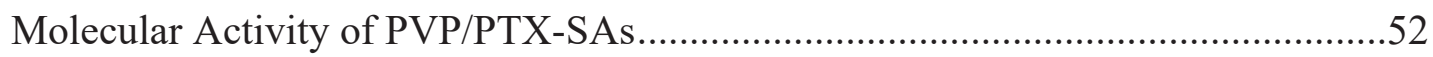

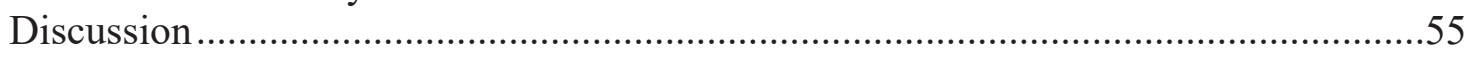

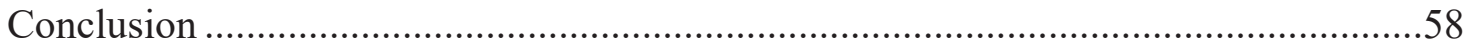

\section{CHAPTER 3. TANNIC ACID-INSPIRED PTX NANOPARTICLES FOR} ENHANCED ANTICANCER EFFECTS IN BC CELLS ...........................................60

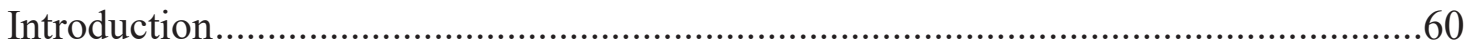

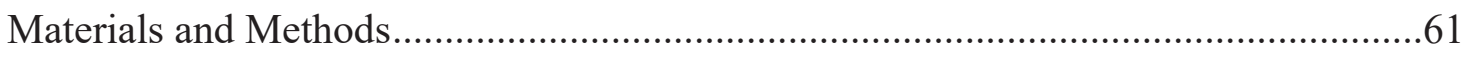




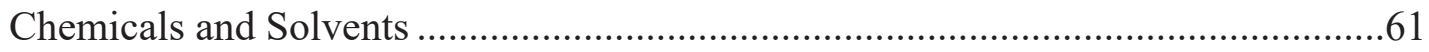

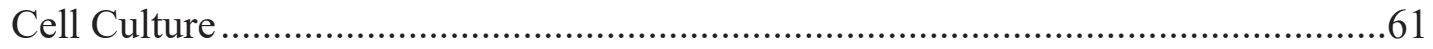

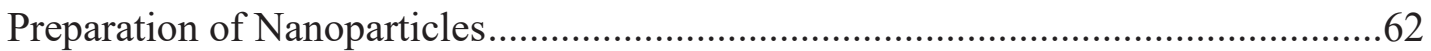

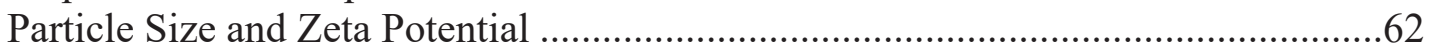

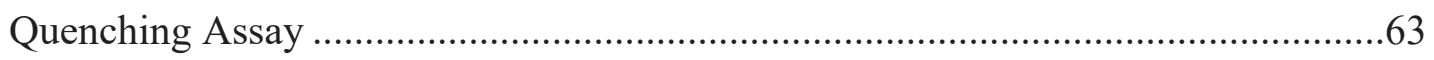

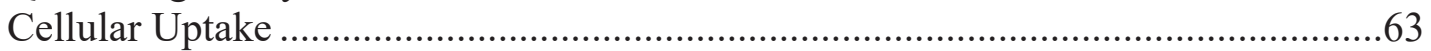

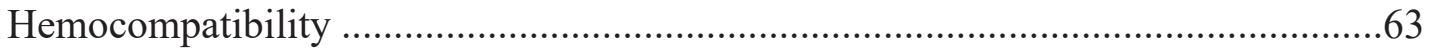

Physiochemical Characterization of TAP NP Formulation .........................................64

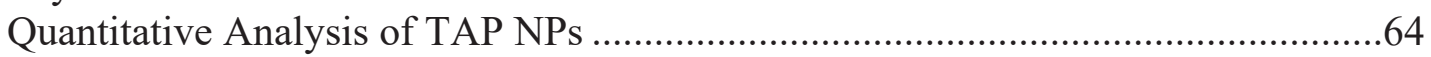

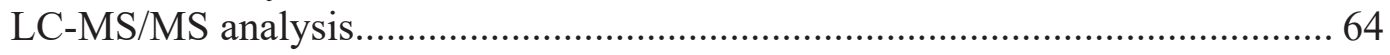

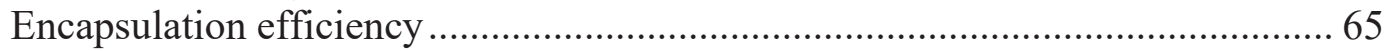

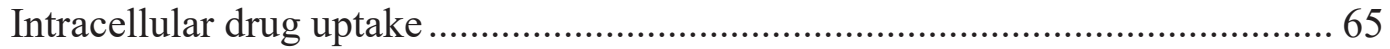

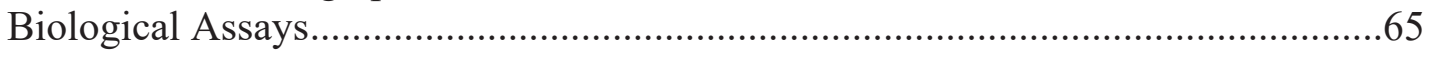

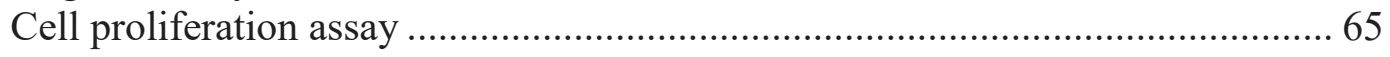

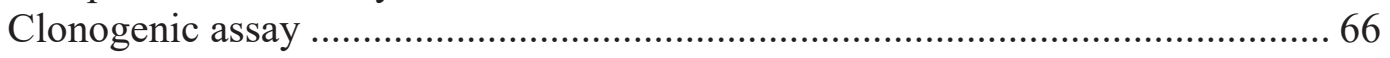

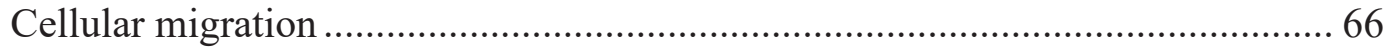

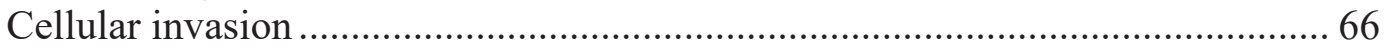

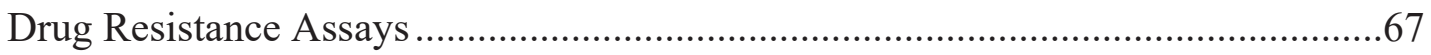

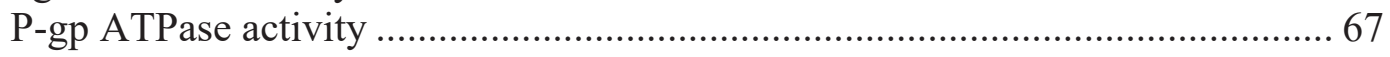

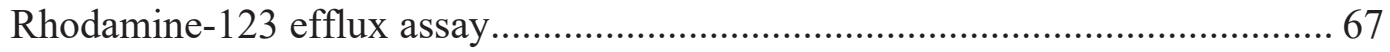

-tubulin Stabilization..................................................................................67

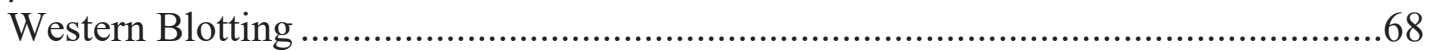

RNA Extraction and Microarray Studies ................................................................68

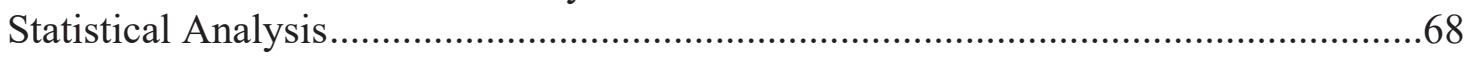

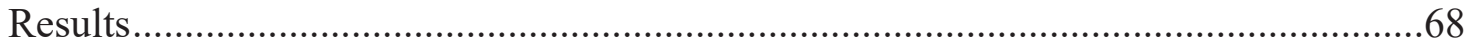

Screening of Pharmaceutical Excipients for PTX Delivery........................................68

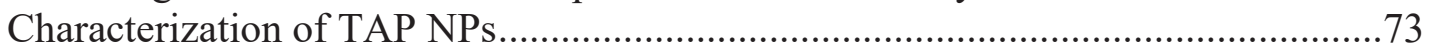

Encapsulation Efficiency and Intracellular Drug Uptake of TAP NPs.......................76

TAP NPs Inhibits Proliferation, Clonogenic Formation, Migration and Invasion

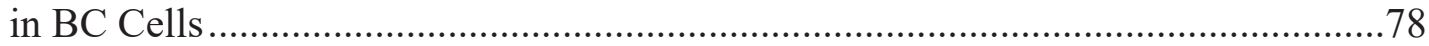

TAP NPs Lower P-gp Expression in BC Cells .......................................................78

Effect of TAP NPs on Microtubule Stabilization, Oncogenic Protein Expression,

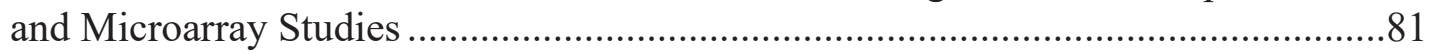

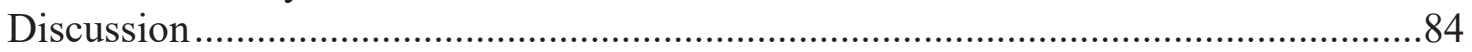

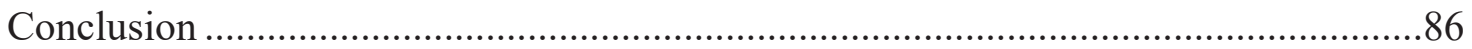

\section{CHAPTER 4. BIO-MEMBRANE INSPIRED NANOPARTICLES FOR} ENHANCED THERAPEUTIC EFFICACY IN TNBC ...........................................89

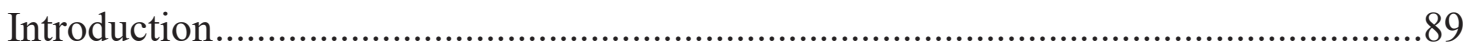

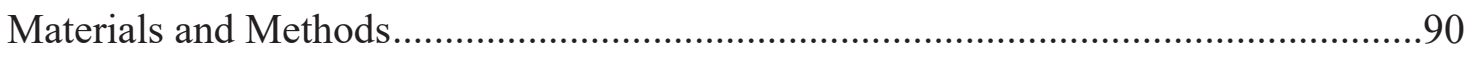

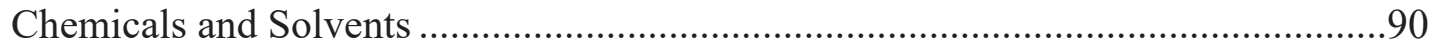

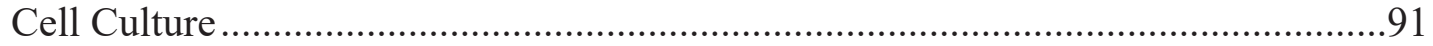

Activation and Isolation of Cell Membrane.............................................................91

Preparation of Polyvinylpyrrolidone-Tannic Acid (PVT) and Membrane-Coated (PVT-MEM) Nanoparticles (NPs) .........................................................................91 
Characterization of PVT and PVT-MEM NPs (PVT-NEU, PVT-NK, PVT-MSC,

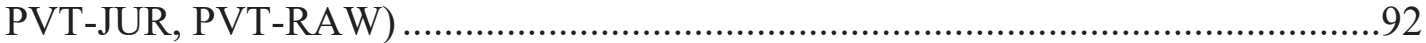

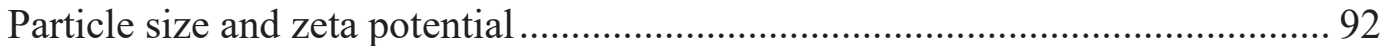

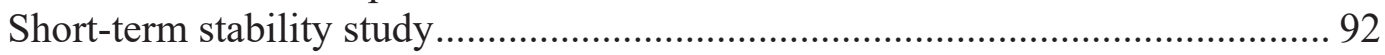

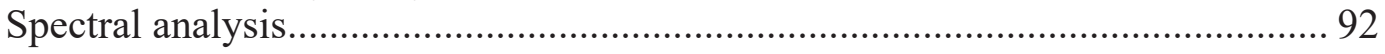

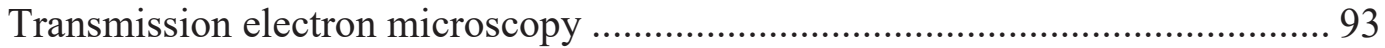

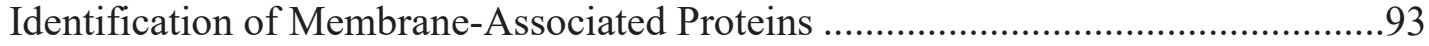

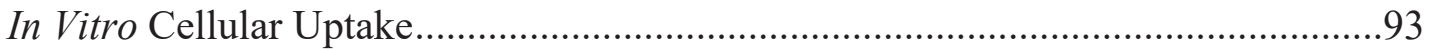

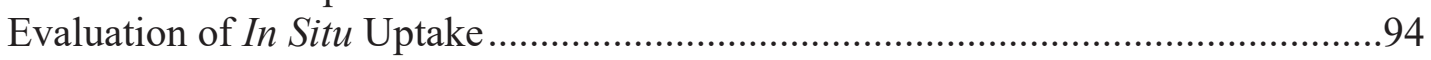

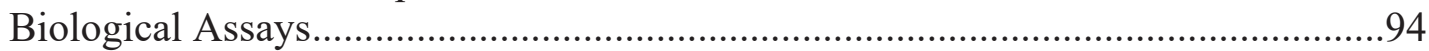

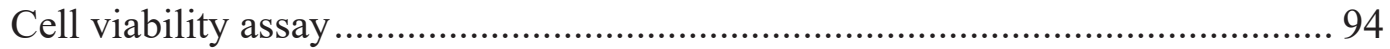

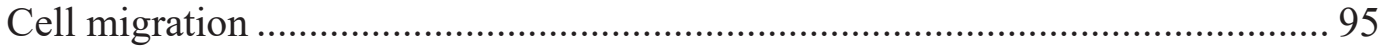

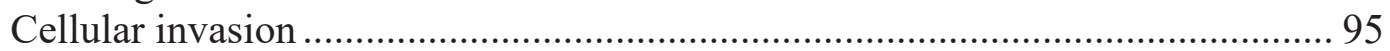

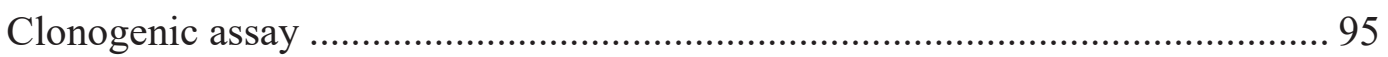

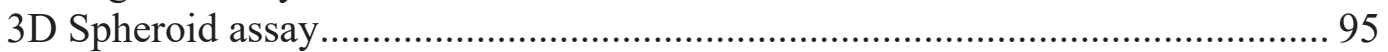

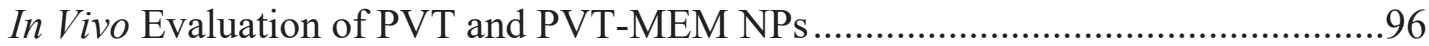

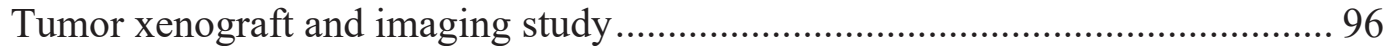

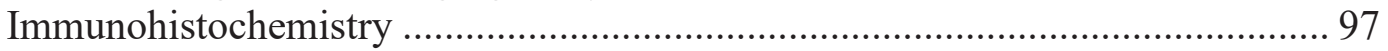

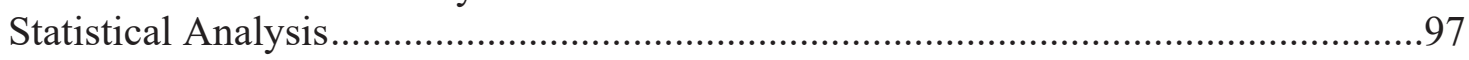

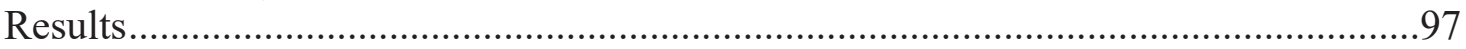

Preparation and Characterization of PVT and PVT-MEM NPs (PVT-NEU, PVT-

NK, PVT-MSC, PVT-JUR and PVT-RAW) .....................................................97

Intracellular and In Situ Uptake and In Vitro Proliferation, Migration, Invasion

and Colony Forming Assay in BC Cells ............................................................... 98

In Vivo Imaging, Biodistribution, Tumor Retention and Efficacy Studies ..............107

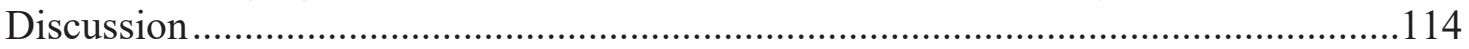

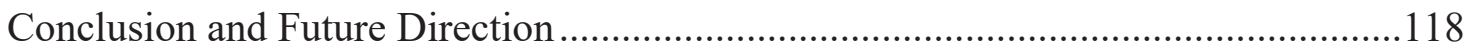

CHAPTER 5. CONCLUSIONS AND FUTURE PERSPECTIVES .........................119

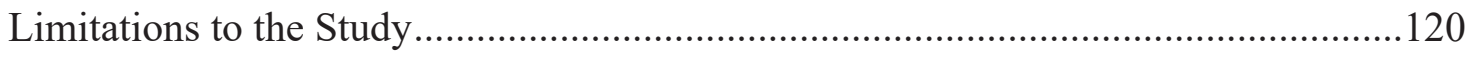

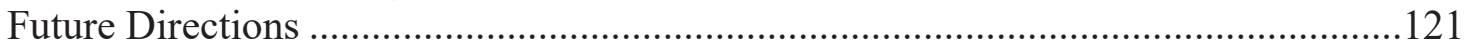

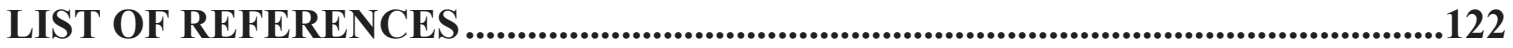

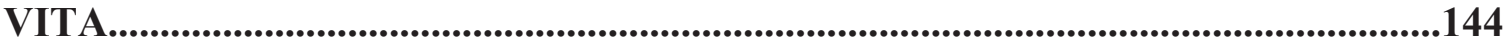




\section{LIST OF TABLES}

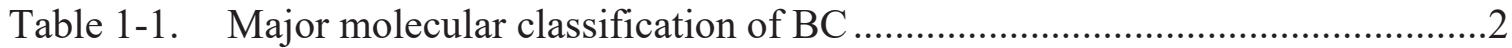

Table 1-2. PTX-based cell membrane coated nanoparticles for therapeutic efficiency/ targeting cancer ..................................................................... 17

Table 2-1. Polymers employed for self-assembling PTX to produce polymer/PTX self-assembly nanoparticles....................................................................40

Table 3-1. Preliminary evaluation of pharmaceutical excipient-based paclitaxel nanoparticles for developing $\mathrm{BC}$ therapeutics........................................70 


\section{LIST OF FIGURES}

Figure 1-1. Schematic illustration of various treatment strategies for TNBC .................6

Figure 1-2. Schematic illustration of the preparation of cell membrane coated nanoparticles. .16

Figure 1-3. Strategies to coat the surface of nanoparticles with cell membrane coating .25

Figure 2-1. Particle size and zeta potential of Poly/PTX-SAs....................................47

Figure 2-2. Evaluation of hemocompatibility behavior of Poly/PTX-SAs ....................48

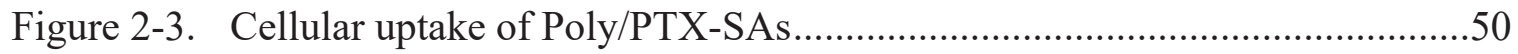

Figure 2-4. Self-assembly formation of PVP/PTX-SAs ...........................................51

Figure 2-5. PVP/PTX-SAs significantly inhibit proliferation of BC cells ....................53

Figure 2-6. PVP/PTX-SAs repress the clonogenic potential of BC cells.......................54

Figure 2-7. Preferential cell death induced by PVP/PTX-SAs in MDA-MB-231 cells ..56

Figure 2-8. Schematic and hypothetical structural representation of PVP/PTX-SAs .....59

Figure 3-1. Characterization during the screening process ...................................... 71

Figure 3-2. Formation and optimization of TAP NPs …...................................... 72

Figure 3-3. In vitro screening during screening process..........................................74

Figure 3-4. Physio-chemical characterization of TAP NPs ........................................ 75

Figure 3-5. Intracellular uptake of TAP NPs in BC MDA-MB-231 cells ......................77

Figure 3-6. Anticancer efficacy of TAP NPs in BC cells ...........................................79

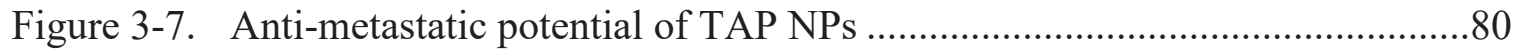

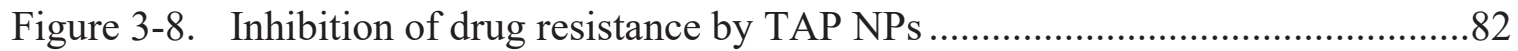

Figure 3-9. Molecular mechanism of TAP NPs in inducing apoptosis in BC cells ........83

Figure 3-10. Schematic representation of TAP NPs inhibiting P-gp efflux .....................88

Figure 4-1. Characterization of PVT and PVT-MEM NPs (PVT-NEU, PVT-NK, PVT-MSC, PVT-JUR and PVT-RAW) 
Figure 4-2. Evaluate the cloaking of cell extracted membrane on to the nanoparticles and the inclusion of PTX in the resultant PVT and PVT-MEM NPs

Figure 4-3. Intracellular uptake PVT and PVT-MEM NPs.............................................103

Figure 4-4. In situ dye uptake by PVT and PVT- MEM NPs........................................104

Figure 4-5. Cell proliferation assay by PVT and PVT-MEM NPs in TNBC cells........104

Figure 4-6. Anticancer efficacy of PVT and PVT-MEM NPs in TNBC cells ..............105

Figure 4-7. Spheroid forming ability of TNBC versus breast epithelial cells ................106

Figure 4-8. In vivo tumor immunofluorescence retention of PVT and PVT-MEM NPs loaded with Indocyanine green ...........................................................108

Figure 4-9. Ex vivo immunofluorescence of excised tumors and organs ......................109

Figure 4-10. Tumor growth inhibition profile of PVT and PVT-MEM NPs (PVTNEU, PVT-MSC, PVT-RAW NPs)

Figure 4-11. Evaluation of potential toxicity of PVT and PVT-MEM NPs PVT-NEU, PVT-MSC, PVT-RAW NPs)

Figure 4-12. Immunohistochemistry analysis and histological evaluation of tumors treated with PVT and PVT-MEM NPs PVT-NEU NPs.

Figure 4-13. Schematic representation of the preparation of cell membranecamouflaged nanoparticles 


\section{LIST OF ABBREVIATIONS}

$\begin{array}{ll}\text { ADC } & \text { Antibody-drug conjugates } \\ \text { ADCC } & \text { Antibody-dependent cellular cytotoxicity } \\ \text { AODNs } & \text { Antisense oligodeoxynucleotides } \\ \text { AR } & \text { Androgen receptor } \\ \text { BBB } & \text { Blood brain barrier } \\ \text { BC } & \text { Breast Cancer } \\ \text { BCS } & \text { Biopharmaceutical classification system } \\ \text { BDS } & \text { Biometric delivery system } \\ \text { BRCA1, BRCA2 } & \text { BC type 1 and 2 susceptibility protein } \\ \text { C6 } & \text { Coumarin 6 } \\ \text { CDK } & \text { Cyclin-Dependent Kinase } \\ \text { CEL } & \text { Cremophor EL } \\ \text { CNS } & \text { Central nervous system } \\ \text { CTLA-4 } & \text { Cytotoxic T-lymphocyte antigen 4 } \\ \text { DMEM } & \text { Dulbecco's Modified Eagle Medium } \\ \text { DOX } & \text { Doxorubicin } \\ \text { DPPC } & \text { Dipalmitoylphosphatidylcholine } \\ \text { DSPE-PEG-MAL } & \text { 1,2-Distearoyl-sn-glycero-3-phosphoethanolamine-N- } \\ & \text { [maleimide(polyethylene glycol)-3400] } \\ \text { DTX } & \text { Docetaxel } \\ \text { EE } & \text { Encapsulation or entrapment efficiency } \\ \text { EGFR } & \text { Epidermal growth factor receptor } \\ \text { ELP } & \text { Elastin-like polypeptides } \\ \text { EMT } & \text { Epithelial-mesenchymal transition } \\ \text { EPR } & \text { Enhanced permeability and retention } \\ \text { ER } & \text { Estrogen receptor } \\ \text { EZH2 } & \text { Enhancer of zeste homolog 2 } \\ \text { FDA } & \text { US Food and Drug Administration } \\ \text { FTIR } & \text { Fourier Transform Infrared } \\ \text { GNRs } & \text { Gold nanorods } \\ \text { GPNMB } & \text { Glembatumumab vedotin } \\ \text { HEPES } & \text { 4-(2-Hydroxyethyl) piperazine-1-ethanesulfonic acid, N-(2 } \\ & \text { Hydroxyethyl) piperazine-N'-(2-ethanesulfonic acid) } \\ \text { HER2 } & \text { Human epidermal growth factor receptor 2 } \\ \text { IBBI } & \text { Interstate Blood Bank, Inc. } \\ \text { IC } 50 & \text { In Vitro 50\% cell growth inhibitory concentration } \\ \text { ICG } & \text { Indocyanine dye } \\ \text { LC-MS/MS } & \text { Liquid chromatography-tandem mass spectrometry } \\ \text { LIV-1 } & \text { Oestrogen-regulated gene } \\ \text { LRP-1 } & \text { Lipoprotein receptor-related protein } \\ \text { MAPK } & \text { Mitogen-activated protein kinases/ extracellular signal regulated } \\ \text { MDR } & \text { Multance } \\ & \end{array}$




\begin{tabular}{|c|c|}
\hline MMAE & Monomethyl auristatin E \\
\hline MRI & Magnetic resonance imaging \\
\hline MTX & Methotrexate \\
\hline NDA & New drug application \\
\hline NSCLC & Non-small cell lung cancer \\
\hline PARP-1 & Poly (ADP-ribose) polymerase \\
\hline PCI & Protease inhibitor cocktail \\
\hline PCL & Poly (caprolactone)-ester endcap polymer \\
\hline PCL-PEG-PCL & Polyethyleneglycol-poly(epsilon-caprolactone) \\
\hline pCR & Pathological complete response \\
\hline PD-1 & Programmed death-1 \\
\hline PDB & $\begin{array}{l}\text { Block-poly[(1,4-butanediol)-diacrylate- } \beta-\mathrm{N}, \mathrm{N} \\
\text { diisopropylethylenediamine] }\end{array}$ \\
\hline PDI & Size distribution/polydispersity \\
\hline PD-L1 & Programmed death ligand 1 \\
\hline PDT & Photodynamic therapy \\
\hline PEG & Polyethylene glycol \\
\hline PEG-PGlu & Poly (ethylene glycol)-poly (glutamic acid) \\
\hline PE-NPs & Coumarin 6 was loaded in pharmaceutical excipient nanoparticles \\
\hline PE-PTX NPs & Pharmaceutical excipient-PTX nanoparticles \\
\hline PI3K/AKT & Phosphatidylinositol 3- kinase \\
\hline PLGA & Poly (lactic-co-glycolic acid) polymer \\
\hline Poly/PTX-SAs & Polymer-PTX-self-assemblies \\
\hline $\mathrm{PR}$ & Progesterone receptor \\
\hline PTX & Paclitaxel \\
\hline $\mathrm{RBCs}$ & Red blood cells \\
\hline RES & Reticuloendothelial system \\
\hline RGD & Arg-Gly-Asp \\
\hline RH123 & Rhodamine-123 \\
\hline RLU & Relative light units \\
\hline $\mathrm{RP}$ & Retinoblastoma protein \\
\hline SDS & Sodium dodecyl sulfate \\
\hline SEM & Standard error of mean \\
\hline TAMs & Tumor-associated macrophages \\
\hline TANs & Tumor-associated neutrophils \\
\hline TAP NPs/ TAP & oarticles $\quad$ TA-bound PTX nanoformulation \\
\hline TCIPA & Tumor cell-induced platelet aggregation \\
\hline TEM & Transmission electron microscope \\
\hline TGA & Thermogravimetric analysis \\
\hline TNBC & Triple negative breast cancer \\
\hline TPGS & D-tocopheryl polyethylene glycol succinate \\
\hline TRAIL & Tumor-specific apoptosis inducing ligand cytokine \\
\hline Trop2 & Trophoblast antigen 2 \\
\hline VEGF & Vascular endothelial growth factor \\
\hline WBCs & White blood cells \\
\hline
\end{tabular}




\section{CHAPTER 1. INTRODUCTION}

\section{Breast Cancer (BC)}

Breast cancer $(\mathrm{BC})$ remains the most commonly diagnosed cancer among women and is the second leading cause of death after lung cancer in the United States [1]. In 2020, it is

estimated that 276,480 new cases of invasive BC will be diagnosed among women, among which 42,170 women are expected to die.

\section{BC Anatomy Causes and Risks}

The anatomy of the human breast is made up of 15-20 lobes, that further branches into smaller lobules and interlobular ducts, that are responsible for transporting the milk from the lobules to the nipples, in nursing women. These ducts and lobules are composed of an inner luminal layer and outer myoepithelial or basal cells. BC begins when the malignant epithelial cells accumulate and grow out, depending on the region they grow out it is classified into noninvasive and invasive. Non-invasive (carcinoma in-situ) is when the carcinoma is confined in the lobules and duct. Invasive carcinomas occur when the carcinoma diffuses to the surrounding connective tissues and metastasize to distant sites. These carcinomas are divided into ductal and lobular carcinoma, depending on where the tumor is formed. Ductal carcinomas comprise twothird of the total $\mathrm{BC}$ and arise from the epithelial cells of the ducts, whereas the lobular carcinoma arises from the lobules and is about one-third of the total BC cases [2].

Some inevitable risk factors include inherited mutations such as BC type 1 and 2 susceptibility protein (BRCA1), BRCA2, atypical hyperplasia, high breast tissue density, older age and/or family history. Race and ethnicity also play a role in the development of BC over a person's lifetime. Other risk factors include weight gain, obesity, menopausal hormone therapy cigarette smoking, alcohol, consumption and physical inactivity [3].

\section{BC Major Molecular Classification}

$\mathrm{BC}$ is a heterogeneous disease and can be classified based on the expression of different biomarkers, exhibiting diversity in biological characteristics and clinical behavior. The expression of four biomarkers, estrogen receptor (ER), progesterone receptor (PR), and human epidermal growth factor receptor 2 (HER2) and Ki-67 classify BC into four main categories, as shown in the Table 1-1 [4].

\section{The estrogen receptor (ER)}

Estrogen is a steroid hormone that is responsible for the growth and bone metabolism, development of mammary glands, as well as maintenance of the central nervous system (CNS) and cardiovascular system. These effects are mediated through a nucleus-initiated 
Table 1-1. Major molecular classification of BC

\begin{tabular}{|c|c|c|c|c|}
\hline Subtype & $\begin{array}{l}\text { Biomarker } \\
\text { expression }\end{array}$ & $\begin{array}{c}\text { Histological } \\
\text { properties \& } \\
\text { prognosis }\end{array}$ & $\begin{array}{l}\text { Available } \\
\text { treatment }\end{array}$ & Percentage \\
\hline Luminal A & $\begin{array}{l}\text { ER+, PR+/-, } \\
\text { HER2- and Ki67 } \\
\text { low }\end{array}$ & $\begin{array}{l}\text { Classic, tubular, } \\
\text { lobular and } \\
\text { cribriform } \\
\text { carcinoma. } \\
\text { Low grade } \\
\text { invasive ductal } \\
\text { carcinoma. } \\
\text { Good prognosis. }\end{array}$ & $\begin{array}{l}\text { Best response to } \\
\text { hormonal } \\
\text { therapy, } \\
\text { Variable } \\
\text { response to } \\
\text { chemotherapy }\end{array}$ & $30-70 \%$ \\
\hline Luminal B & $\begin{array}{l}\text { ER+, PR+/-, } \\
\text { HER2+/- and } \\
\text { Ki67 high }\end{array}$ & $\begin{array}{l}\text { Micropapillary } \\
\text { carcinoma. } \\
\text { Invasive ductal } \\
\text { carcinoma. } \\
\text { More aggressive } \\
\text { than Luminal A. } \\
\text { Prognosis is not } \\
\text { as well } \\
\text { established as } \\
\text { Luminal A. }\end{array}$ & $\begin{array}{l}\text { Hormonal } \\
\text { therapy in } \\
\text { combination } \\
\text { with anti-HER2 } \\
\text { therapy } \\
\text { (Trastuzumab), } \\
\text { depending on the } \\
\text { expression of } \\
\text { HER2, Variable } \\
\text { response to } \\
\text { chemotherapy. }\end{array}$ & $10-20 \%$ \\
\hline HER2 positive & $\begin{array}{l}\text { ER-, PR- and } \\
\text { HER2+ }\end{array}$ & $\begin{array}{l}\text { High grade } \\
\text { invasive ductal } \\
\text { carcinoma. } \\
\text { Poor prognosis. }\end{array}$ & $\begin{array}{l}\text { Responds well } \\
\text { with anti-HER } 2 \\
\text { therapy, and } \\
\text { chemotherapy }\end{array}$ & $15-20 \%$ \\
\hline $\begin{array}{l}\text { Triple-negative } \\
\text { breast cancer } \\
\text { (TNBC) }\end{array}$ & $\begin{array}{l}\text { ER-, PR- and } \\
\text { HER2- }\end{array}$ & $\begin{array}{l}\text { High-grade } \\
\text { invasive, } \\
\text { Medullary, and } \\
\text { metaplastic } \\
\text { carcinoma. } \\
\text { Worst prognosis }\end{array}$ & $\begin{array}{l}\text { Chemotherapy is } \\
\text { the only } \\
\text { treatment. } \\
\text { Olaparib and } \\
\text { Atezolizumab } \\
\text { have recently } \\
\text { been approved } \\
\text { by the FDA for } \\
\text { TNBC patients } \\
\text { with BRCA1/2 } \\
\text { mutation and } \\
\text { PD-L1 positive, } \\
\text { respectively. }\end{array}$ & $5-15 \%$ \\
\hline
\end{tabular}

Sources: Onitilo, A. A., Engel, J. M., Greenlee, R. T., \& Mukesh, B. N. (2009). Breast cancer subtypes based on ER/PR and Her2 expression: comparison of clinicopathologic features and survival. Clinical medicine \& research, 7(1-2), 4-13 [5], \& Weigelt, B., Peterse, J. L., \& Van't 


\section{Table 1-1. (Continued)}

Veer, L. J. (2005). Breast cancer metastasis: markers and models. Nature reviews cancer, 5(8), 591-602. 
estrogen receptor, which belongs to the nuclear hormone receptor superfamily. Around 70-75\% of the total BC cases are ER-dependent. They can either be Luminal A or Luminal B, varying on the expression of proliferation associated genes. Overall, both the subtypes of ER+ BC; Luminal $\mathrm{A}$ and $\mathrm{B}$ have a good prognosis with excellent long-term survival. Treatment options available for ER-dependent pathway in the form of:

- Selective ER agonist Tamoxifen, that inhibits BC cells by competitive antagonism with estrogen at the binding site. In normal breast, it behaves as an antagonist and inhibits the growth of cancer cells, although it functions as an agonist in the bone and the endometrium [6].

- Selective ER inhibitor Fulvestrant, due to the absence of any estrogenic activity, functions far more effectively than Tamoxifen and is able to prolong the effect of the treatment and also reduce the chances of developing drug resistance, which remains a major hindrance in tamoxifen therapy [7].

- Aromatase inhibitors Letrozole and Anastrazole, suppress the activity of aromatase enzyme, preventing the conversion of androgen to estrogen especially in menopausal women. Aromatase inhibitors in $\mathrm{BC}[8]$.

\section{Human epidermal growth factor receptor 2 (HER2)}

HER2 belongs to the human epidermal growth factor receptor (HER) family that is responsible for cell growth, survival, cell differentiation, and proliferation. It exerts oncogenic behavior due to the overexpression of the HER2 gene [9]. HER2 directed therapy although exerted increased risk of disease-free survival and poorer overall survival. However, it responds well to anti-HER2 therapy such as a monoclonal antibody, Trastuzumab, and dual EGFR and HER2 receptor tyrosine kinase inhibitors such as Lapatinib [10]. Trastuzumab in combination with chemotherapy improves cancer recurrence rates and overall survival by $50 \%$ and $33 \%$ respectively in contrast to chemotherapy alone [11].

\section{Progesterone receptor (PR)}

PR plays a vital role in mammary epithelial cell proliferation and differentiation [12]. The effect of PR on cancer cells is controversial. It has been reported that the effects of PR on cancer cells causes binding of nuclear PR and translocating of the PR complex to the nucleus, thus causing activation of the target genes [13]. At the same time, reports have been available showing that inclusion of progesterone during hormone therapy for $\sim 5.6$ years results in increased risks in $\mathrm{BC}$ with intervention. However, even after cessation of therapy chances of risk remains high several years after stopping the regimen. Researchers have shown may be the level of progesterone in the stem cell niche could initially mitigate the chances of early cancer lesions during the early post-intervention period. Although the risk of $\mathrm{BC}$ is an elevated postintervention period as cancer has probably progressed to an advanced stage prior to treatment cessation and would no longer be dependent on progesterone treatment [14]. Although further studies are being explored to pinpoint the role and mechanistic impact of progesterone for 
progression of $\mathrm{BC}$, that could shine light on the therapeutic intervention for advanced stage triple-negative breast cancer

\section{Triple-negative breast cancer (TNBC)}

TNBC represents the most aggressive and heterogeneous subtype of BC. It is characterized by a lack of expression of ER, PR, and HER2 overexpression. Typically, TNBC demonstrates an increased mitotic activity, increased expression of proliferation markers such as Ki67, high nuclear atypia, high nuclear-cytoplasmic ratio, scant stromal content, central necrosis, multiple apoptotic cells, invasive and stromal lymphocytic infiltration [15, 16]. Additionally, tumor growth is rapid, of a higher grade, greater chances of lymph node involvement and metastasizes mostly to the viscera, especially the lungs and the brain [17]. The distinct cellular phenotype, aggressive nature, metastatic potential and lack of receptor or targeted therapy, makes chemotherapy the only treatment modality for TNBC. However, TNBC with the worst prognosis and high recurrence rate within the first 5 years of therapy [18] and shorter overall survival of only 9-13 months [19], in contrast to other subtypes of BC, makes therapeutic intervention for TNBC an unmet need.

\section{Therapeutic Strategies for TNBC, Evaluated by Clinical Trials}

As described above, TNBC due to heterogeneity and molecular variability there was no specific protocol for this type of disease. Although the relentless efforts of researchers have led to an improvement in the therapy of TNBC, and newer targets are discovered by studying the genomic variability. Therapies that have shown promising outcomes in TNBC, either alone or in combination with neoadjuvant therapy that has led them to be evaluated for clinical trial are summarized below. Figure 1-1 shows the schematic representation of the various treatment strategies.

\section{Conventional Chemotherapy for TNBC}

Chemotherapy with cytotoxic drugs remains the mainstay treatment for TNBC. These are usually agents such as anthracycline, platinum and/or taxanes either in the neoadjuvant or adjuvant setting. Anthracyclines such as doxorubicin and epirubicin have shown enhanced improvement in the response rate of survival by 22 months [20]. These agents have proven to be beneficial and have shown enhanced sensitivity both when used as a single agent [21, 22] as well as in neoadjuvant setting [23]. Phase III clinical trial of anthracyclines as an adjuvant agent versus CMF (cyclophosphamide, methotrexate and 5-fluororacil) by Di et. al. demonstrates that a $23 \%$ decrease in the recurrence rate [24]. A 17\% pathological complete response (pcR) in a Phase II clinical trial was observed in a neoadjuvant setting with anthracyclines agents in combination with others such as cyclophosphamide, 5-fluororacil and epirubicin [25]. Gluz et al. demonstrate in a Phase III clinical trial that 5-year disease-free survival was achieved in $71 \%$ of the TNBC patients treated thrice every week in contrast to only $26 \%$ improvement with treated twice weekly when anthracyclines are used in combination [26]. Taken together, although a higher response rate $[27,28]$ is observed in TNBC patients, higher recurrence rates and low 


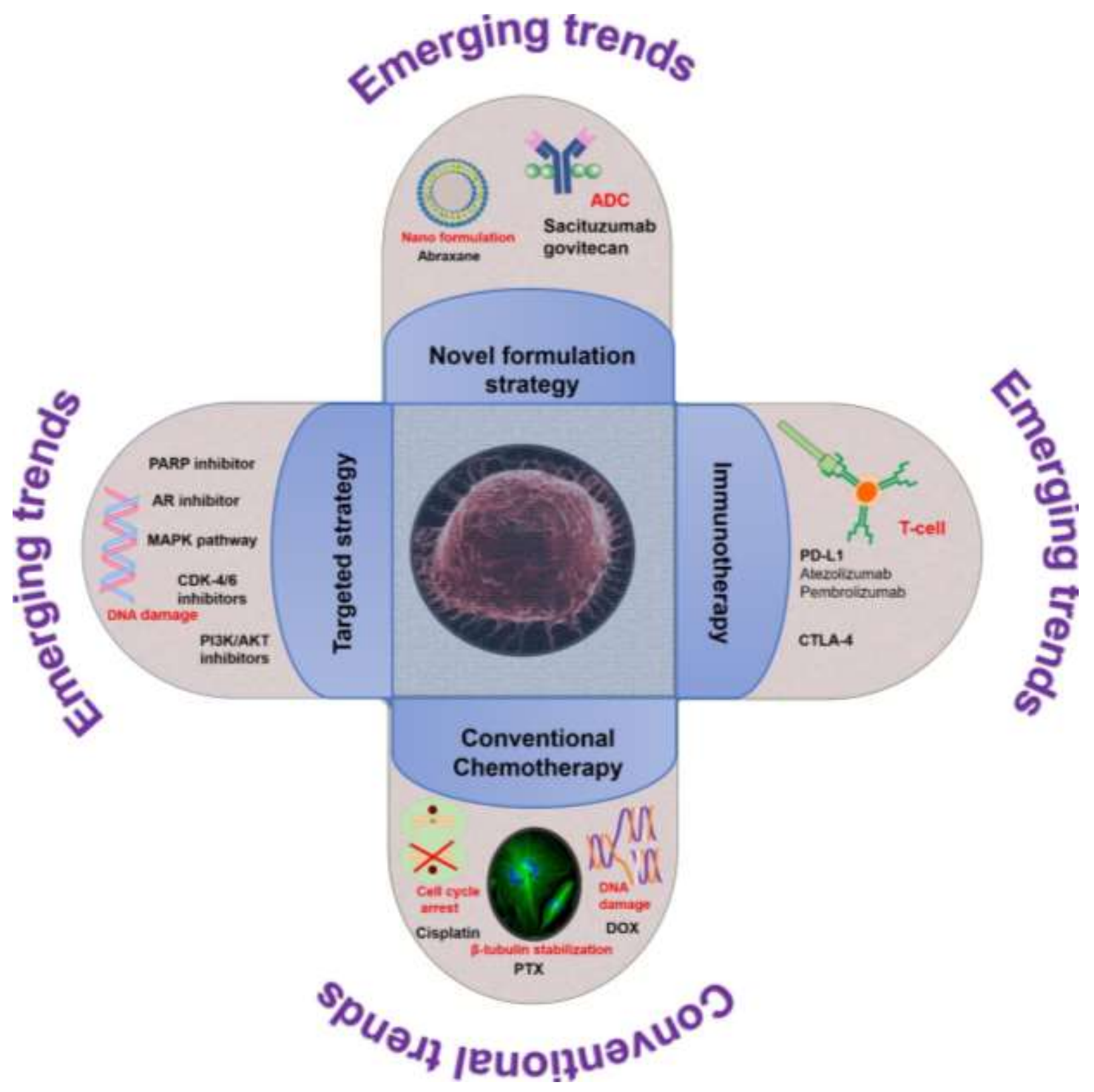

Figure 1-1. Schematic illustration of various treatment strategies for TNBC

Although chemotherapy remains the backbone for the treatment for most of TNBC patients with cytotoxic agents, yet emerging trends are evolving. These mostly include immunotherapy (checkpoint inhibitors), targeted therapy (inhibitors of various pathways) and novel formulation strategies (neoformation, ADC). 
overall survival [29], has limited the usage of response rate as a determinant for therapeutic superiority. Other associated side effects include acute toxicity such as irreversible cardiotoxicity myelotoxicity, alopecia, nausea, and vomiting [29-31] with these agents.

Platinum agents have proven to be beneficial for the treatment of TNBC patients with a BRCA mutation when used in combination as well as single agents [32]. A Phase II clinical trial (NCT00148694) at Dana Farber Cancer Institute suggested that 21\% of patients received pcR with single-agent cisplatin and 15\% response when excluding the BRCA1 mutations suggesting the importance of platinum agents as adjuvant or neoadjuvant therapy in BRCA mutants [21]. BRCA1 mutant has shown increased sensitivity to platinum agents because of the ability to repair DNA, however, it may become resistant and may be required to be provided in homologous combination [22]. A Phase II clinical trial suggests carboplatin and PTX with or without trastuzumab showed a 67\% increase in pcR [33]. In another Phase II clinical trial cisplatin combination with epirubicin and fluorouracil in addition to PTX versus without the PTX treatment improved the response rate by $40 \%$ [34]. A higher response rate of 34\% was observed in women treated with platinum and docetaxel [35]. Thus, a combination of platinum agents has shown to have higher efficacy than when used alone. Although therapy with platinum agents are of special interest when treating a TNBC patient with a BRCA defect.

Taxanes either as a single agent or in combination with anthracyclines have shown to be beneficial in TNBC subtype in contrast to other subtypes of BC, attaining pcR of up to 40\% [36]. Cancer and Leukemia Group B (CALGB) conducted a trial on 9344 subjects, demonstrate that there was particularly improvement in disease-free survival in TNBC patients treated PTX versus in all other subtypes of BC [37]. BC International Research Group (BCIRG) led another 001 trial and particularly yielded an advantage in the group that was cotreated with PTX over cyclophosphamide, suggesting that there is significant inhibitory effect of PTX especially on the TNBC cohort [38]. Further, a non-platinum-based neoadjuvant therapy with cyclophosphamidevincristine-doxorubicin-prednisolone shows a $15 \%$ increase in the pcR in comparison to all the other regimen, suggesting TNBC apart BRCA mutation subset, have greater with taxanes in comparison to other regimens [39].

\section{Targeted Therapies}

The molecular heterogeneity of TNBC makes it difficult for physicians and clinicians to have a standard care guided approach for the treatment. Although, advancement in the molecular classification and genome sequencing, the development of targeted therapy is improving. From a variety of gene expression analysis, six main subtypes of TNBC have been proposed, viz. basallike 1, basal-like 2, immunomodulatory, mesenchymal, mesenchymal stem-like, and luminal androgen receptor (AR) [40]. Numerous clinical trials are ongoing to investigate a variety of targeted therapy for TNBC, such as PARP inhibitors, phosphatidylinositol 3- kinase (PI3K/AKT) inhibitors, mitogen-activated protein kinases/ extracellular signal-regulated kinases (MAPK) and cyclin-Dependent Kinase (CDK) 4/6 inhibitors.

Poly(ADP-ribose) polymerase (PARP-1) inhibitor leads to an increase in the doublestrand DNA causing an increase in apoptosis [41]. PARP-1 inhibitor has been particularly 
beneficial in BRCA1 and BRCA2 mutant cells because of their ability to sensitize cells [42, 43]. Although BRCA mutations account for only $20 \%$ in TNBC population, yet several PARP inhibitors have gained clinical approval such as olaparib, veliparib, niraparib, rucaparib and talazoparib. In a phase III clinical trial in TNBC patients with BRCA mutation demonstrate that oral PARP inhibitor olaparib increases the progression-free survival to 7 months in contrast to 4.2 months in the placebo group. US Food and Drug (FDA) recently in January 2018 approved the first PARP inhibitor: olaparib for the treatment of advanced-stage BRCA mutant TNBC [44]. talazoparib was used as monotherapy in the neoadjuvant setting in a phase II clinical trial: NCT02282345, for patients with BRCA mutations. Among the twenty patients enrolled, seventeen were TNBC positive. After 6 months of treatment followed by surgery and adjuvant chemotherapy, 53\% of patients achieved pCR [45]. Currently, a larger single-arm Phase II study is ongoing (NCT03499353) by Pfizer. Also, EMBRACA study conducted on HER2 negative advanced BC improved the overall survival to 24.3 months, in contrast to only 6.3 months in patients receiving only chemotherapy. This lead to the approval of the second PARP inhibitor, Talazoparib for germline BRCA mutation in October 2018 [46].

The PI3K/AKT regulates cell growth and glucose metabolism. There are complexities associated with the activated PI3K/AKT pathway, although activated PI3K/AKT pathway is high in TNBC [46]. Ipatasertib, an oral AKT inhibitor is used in a LOTUS (NCT02162719), a phase II clinical trial in TNBC patients, in combination with PTX demonstrated improved progressionfree survival from 9 months to 4.9 months, with PTX [47], with also improvement in overall survival of 23.1 months versus 18.4 months with PTX [48]. Recently, an ongoing clinical trial phase II with combination of PTX and ipatasertib is being conducted as neoadjuvant therapy in stage I-III TNBC (NCT02301988) patients. Inhibition of epidermal growth factor receptor (EGFR), a transmembrane tyrosine kinase receptor by PI3K/AKT pathway has proven to be useful used in the treatment of TNBC. However, cetuximab a humanized monoclonal antibody used in combination with other taxanes failed to show any improvement in the TNBC subtype [49]. In a similar study, of phase II trial by the Translational BC Research Consortium demonstrates that cetuximab used alone or in combination with platinum agents such as cisplatin and carboplatin, failed to show any impressive result by the anti-EGFR antibody [50]. This is probably because only $50 \%$ of the TNBC cases are EGFR positive [51].

AR accounts for 13-37\% among TNBC patients [52], with more prevalence among older people [53]. However, the role of AR as a prognostic marker in TNBC is not clear, as there are contradictory reports about the characteristics of AR in TNBC. Some suggest it is associated with low nuclear grade, and low proliferative rate and is unrelated to be a prognostic marker [54], whereas some suggest they have a higher nuclear grade and high lymph node metastasis and higher mortality rate [55]. Although, there are several clinical trials with AR and have shown promising results. Enzalutamide, is a novel targeted AR inhibitor that competitively binds to the AR and prevents translocation to the nucleus [56]. NCT02750358 is a Phase II ongoing clinical trial for determining the compliance rate of enzalutamide as adjuvant therapy for a 1-year dose [57]. In another Phase II, clinical trial demonstrates clinical benefits are achieved and enzalutamide may be a therapeutic option for patients who have received chemotherapy. To further evaluate the efficiency of enzalutamide with PTX a phase IIB is currently ongoing. Bicalutamide also an AR inhibitor, has shown to have promising results in a phase III trial for metastatic TNBC patients [58]. 
The role of activation MAPK was initially investigated in vitro, which causes loss or downregulation of various genetic and epigenetic functions that are associated with chemoresistance. MEK inhibitors are known to cause efficient suppression of chemoresistance either alone or in combination with other chemotherapeutic drugs. Significant inhibitory effects were seen with combination treatment of cobimetinib with PTX [59]. Another study demonstrates, MEK inhibitor selumetinib given in combination with either buparlisib or the platelet-derived growth factor receptor inhibitor pazopanib showed effective inhibition of brain metastases in TNBC patients [60]. An ongoing clinical trial shows MEK inhibitor trametinib in combination with gemcitabine showed complete response in the patient with metastatic TNBC [61]. However, there have been serious toxicities prohibiting phase II clinical study of MEK inhibitor with mTOR or PI3K inhibitors [62, 63].

CDK is responsible for hyperphosphorylation of retinoblastoma protein (RP) and causes G1-S phase transition in the cell cycle [64]. CDK inhibitors thus were explored as a potential target for TNBC. Palbociclib is a selective inhibitor of CDK was explored in clinical trials for TNBC with positive RP expression and demonstrated a partial response in $50 \%$ of the patients, improving disease-free survival for equivalent or more than 6 months [65]. There are several other clinical trials conducted either alone or in combination with other chemotherapy.

\section{Antibody-Drug Conjugates (ADC)}

ADCs are targeted therapy where the toxic anticancer drug targets the cancer cells through the specific binding of an antibody that has specificity for receptors on the surface of cancer cell. There have been lots of ongoing research using ADCs for TNBC with some of them discussed below.

Trophoblast antigen 2 (Trop2) [66] is another target via ADC for TNBC. IMMU-132, also known as sacituzumab govitecan is an ADC that targets Trop2 and have been used in combination with three PARP inhibitor; olaparib, rucaparib and talazoparib and have shown improved overall response [67]. Another compelling ADC, glembatumumab vedotin (GPNMB) that combines the monomethyl auristatin E (MMAE) (a potent microtubule-disrupting agent) shows improvement in disease-free progression in Phase I/II clinical trial [68]. However, finding suggests that a $100 \mathrm{mg}$ dose caused some toxicity of grade 3 and 4, may limit the usage of these agents [69]. Ladiratuzumab vedotin is another ADC explored for targeting TNBC and is composed of a humanized IgG1, MMAE and a monoclonal antibody for targeting oestrogenregulated gene (LIV-1). LIV-1 is overexpressed in 90\% TNBC tumors but is not significantly expressed in normal tissues. In a phase I study with ladiratuzumab vedotin a $25 \%$ overall response rate was achieved. Although there are toxicities issues such as alopecia, neutropenia and peripheral neuropathy. Ongoing studies are still conducted to evaluate the efficiency of ladiratuzumab vedotin as monotherapy or in combination with chemotherapy for TNBC [70]. 


\section{Immunotherapy}

TNBC is known to have the highest mutations [71] among all other types of BC. The role of tumor-infiltrating lymphocytes plays a major role in disease progression in TNBC. Thus, activation of these cytotoxic $\mathrm{T}$ cells would be initiating an antitumor immune response. $\mathrm{T}$ cells show the expression of immune checkpoint inhibitors: cytotoxic T-lymphocyte antigen 4 (CTLA-4) and programmed death-1 (PD-1). PD-1 inhibitors are the most extensively studied checkpoint and is a cell surface protein expressed on the infiltrating tumor lymphocytes that induce inhibition of T cell by binding to two ligands: PD-L1 and PD-L2 [72]. Researchers are actively involved in evaluating the role of these checkpoint inhibitors and have shown some encouraging results for the treatment of TNBC

Among these PD-1 inhibitor, pembrolizumab was evaluated as a first-line therapy among PD-L1 positive TNBC patients. In which $87 \%$ of the patients had received either neoadjuvant or adjuvant chemotherapy, with overall survival outcome being 16.1 months [73]. In another phase II trial, I-SPY 2 trial (NCT01042379), 69 patients were HER- and 29 TNBC patients, received either neoadjuvant PTX and/or pembrolizumab, followed by dose-dependent doxorubicin and cyclophosphamide. The raw pathological complete response was improved to $71 \%$ with pembrolizumab compared to $19 \%$ in the control arm, whereas the estimated pcR was $62 \%$ with pembrolizumab versus $22 \%$ in the control arm [73]. The beneficial results achieved through ISPY 2 trial led to the phase III trial, KEYNOTE-552 (NCT03036488) and KEYNOTE-355 (NCT02819518) which are currently ongoing. Another PD-L1 inhibitor that has achieved impressing outcomes among TNBC patients is, atezolizumab. Impassion130 trial, NCT02425891 which was a phase III trial, among metastatic or advanced stage TNBC patients receiving Abraxane with/without atezolizumab plus placebo. 1.4 fold improvement was achieved among patients receiving atezolizumab, with progression-free survival of 2.5 months, versus 1.7 months who did not receive atezolizumab, overall survival was also improved to 9.5 months, with no significant improvement observed among patients without atezolizumab [74]. Based on these results, the FDA accelerated the approval of atezolizumab with Abraxane for the treatment of metastatic TNBC patients who are PD-L1 positive. Further ongoing phase III trial NCT03125902 is being evaluated with patients receiving either atezolizumab and PTX versus PTX and placebo for first-line therapy in TNBC.

Due to the heterogeneity and the inherent nature of TNBC to develop multi drug resistance (MDR) to conventional chemotherapeutics, several ongoing clinical trials with combination therapies or targeted therapies or immunotherapies or ADC, are being evaluated to identify predictable biomarkers that will improve treatment outcomes among TNBC patients. Although, the paradigm is shifting and lots of attention is being given to the development of novel and nano-based therapies for TNBC.

\section{Nano-Based Therapies for TNBC}

The lack of these cellular receptors makes the delivery of therapeutics for TNBC patients a crucial need. Nanotechnology-based delivery systems have been beneficial to provide significant intratumoral delivery both by active and passive targeting. Passive targeting occurs by 
a phenomenon known as enhanced permeability and retention (EPR) effect. This phenomenon occurs because of the tumor vasculature that is hyper vascularized with enhanced vascular permeability causing fluid retention and lack of lymphatic drainage. This results in extravasation within tumor tissues and increased accumulation of therapeutics at the intertumoral site, whereas they get cleared into the lymphatic system of healthy cells [75]. However, achieving therapeutic concentrations inside the tumor site is a challenge with passive targeting and as a result of which compromising with the biotherapeutic window of the drug. Although, active targeting relies on conjugating the surface of the nanoparticle to biocompatible targeting moieties such as aptamers, antibodies, peptides, that has specificity to the antigens or receptors at tumor site [76, 77]. This approach provides a myriad of advantages over conventional therapeutic approaches, such as:

- $\quad$ Target specific delivery

- Minimizes systemic or non-specific toxicities

- Prolonged biodistribution with intravenous administration

- Increase of therapeutic window, resulting in transport of maximal payload

- Less immunogenic reaction

- Controlled drug release, improving half-life and systemic circulation

- Increase in drug solubility, chemical stability and efficacy of poorly water-soluble chemotherapeutic agents.

- Combined therapy has been able to deliver multiple therapeutic agents by synergistic effects, and useful for multimodal functional, such as imaging and/or theranostic agent [78-80].

Some of the commonly employed nanoparticles that have been explored further are polymeric nanoparticles, micelles, dendrimers, liposomes, nanoconjugates. These systems are further modified for delivering not only drugs but also oligonucleotides, DNA or proteins [81].

\section{Polymeric nanoparticles}

Polymeric nanoparticles are nanosized range materials that are synthesized using either natural or synthetic biodegradable and biocompatible polymers. Depending on the characteristics of the polymer of choice, both hydrophilic and hydrophobic drugs can be encapsulated into these nanoparticles that can be released by surface or bulk erosion, swelling or diffusion. Studies have shown that polymeric nanoparticles made of polyethyleneglycol-poly(epsilon-caprolactone) (PCL-PEG-PCL) encapsulating docetaxel successfully improved the in vivo survival rate of mice in contrast to marketed docetaxel formulation due to the prolonged circulation of the nanoparticles for EPR effect. Similarly, poly(lactic-co-glycolic acid) polymer (PLGA)polyethyleneglycol (PEG) nanoparticles were utilized to deliver cisplatin in TNBC xenograft 
mouse model with significant tumor inhibition and prolongation of half-life, although systemic toxicities were observed in normal organs such as kidney and liver [82].

These loopholes of passive targeting have led into the utilization of nanoparticles by decorating the surface with active moieties allowing longer circulation, causing higher concentration accumulating in tumor tissues compared to normal tissues. Passarella et al. utilized a biology-based screening technique called Phage Display, to identify the glucose-regulated protein (GRP78), a radiation-induced cell surface receptor that is known to have higher pathologic grade and known to cause metastasis, lower chemotherapeutic response, patient survival and cancer prognosis in TNBC [83]. By conjugating a novel peptide (Gly-Ile-ArgLeu-Arg-Gly) to polymeric nanoparticles made of polyester and encapsulating anticancer agent PTX, the authors display targetability at irradiated xenograft TNBC mice model and significant delay in tumor growth in contrast to untargeted nanoparticles [84]. In another approach, a protein polymer called elastin-like polypeptides (ELP) was used to form nanoparticles that assemble into $<100 \mathrm{~nm}$ and was surface decorated with FKBP, which is a cognate receptor for potent yet poorly soluble rapamycin. These nanoparticles prolonged circulation time and increased the halflife by 26 folds, with enhanced anti-tumor efficacy and lower cytotoxicity than free drug in TNBC xenograft mouse model [85]. Enhancer of zeste homolog 2 (EZH2) was found to be overexpressed in TNBC and is found to be responsible for poorer overall patient survival. Delivery of siRNA via chitosan nanoparticles directed to target the EZH2 in the orthotopic MDA-MB-231 TNBC mouse model has shown to cause a significant reduction in tumor growth [86].

\section{Polymeric micelles}

Polymeric micelles are formed using hydrophilic and hydrophobic polymers, that forms Van der Waals bonds to form a hydrophobic core which usually encapsulates the drug either by physical entrapment or chemical bonds. The outer shell on the other hand being hydrophilic, aids prolong circulation and prevents rapid clearance by the reticuloendothelial system (RES) [87]. A block copolymer of poly (ethylene glycol)-poly (glutamic acid) (PEG-PGlu) conjugated to the active metabolite of topoisomerase I inhibitor irinotecan (SN-38) forms polymeric micelles around $20 \mathrm{~nm}$. Significant tumor inhibition in an orthotopic mouse model has made this a promising candidate and is in Phase II clinical trial for TNBC patients [88]. Active targeting by polymeric micelles was designed by Kutty et. al. using D-tocopheryl polyethylene glycol succinate (TPGS) polymer, encapsulating docetaxel conjugated to cetuximab, that specifically targets EGFR on the TNBC tumors by 205.6 and 223.8 folds in contrast to the marketed formulation of docetaxel, Taxotere ${ }^{\circledR}$ [89].

\section{Liposomes}

Liposomes are composed utilizing the lipid bilayer characteristics, which allows the formation of spherical vesicles as large as $400 \mathrm{~nm}$, enclosing an aqueous core. The lipid bilayer being amphiphilic allows the interaction of the hydrophilic surface of the lipid with the central aqueous core-forming lipid spheres, whereas the hydrophobic region can be modified as per drug delivery needs. The ability of drug loading both in the aqueous core or lipid bilayer and the possibility of active and passive targeting makes liposomes an attractive alternative for 
researchers. Researchers have utilized the lipid-based nanocapsules to entrap lipophilic drug ferrocenyl tamoxifen derivative FcOHTAM and demonstrated improved antitumor effects in TNBC xenograft model that is resistant to tamoxifen treatment [90]. Lipid-based nanoparticles were constructed from chemically modified cholesterol-terminated poly (acrylic acid) and crosslinked with diamine linkers for active targeting. The authors demonstrate a high drug loading capability of doxorubicin via this approach exhibiting superior tumor growth inhibition and no systemic toxicity in comparison to free drug in orthotopic TNBC murine model [91]. A similar finding by Ahn et. al. show liposome vesicles encapsulate arsenic trioxide, which due to doselimiting toxicity and rapid clearance was not well explored despite having potential antitumor activity. The stabilized $100 \mathrm{~nm}$ size ranged liposomal vesicles resulted in enhanced antitumor efficacy than parent drug in orthotopic murine model, probably due to the prolonged retention of the drug in the lipid bilayers, improving the pharmacokinetic parameters and efficacy profile [92]. Pegylated liposomal formulation of doxorubicin and gemcitabine showed remarkable responses in TNBC patients, however adverse effects such as metastasis to the skin have limited its use [93]. In an attempt of active targeting, integrin $\alpha 3$ that is overexpressed in TNBC models and is known to cause tumor angiogenesis, proliferation, therapeutic resistance, and poor prognosis, is attached to the surface of PEG-PCL based liposomes. The authors attach a cyclic octapeptide LXY (Cys-Asp-Gly-Phe(3,5-DiF)-Gly-Hyp-Asn-Cys) and achieve enhanced accumulation of co-administered drugs (doxorubicin and rapamycin) at the tumor site, resulting in improved antitumor efficacy in in vivo TNBC model [94]. Active targeting of eEF-2K which has shown to cause tumorigenesis and is associated with a poor prognosis in TNBC, by neutral liposomal siRNA formulation leads to significant inhibition in tumor growth in orthotopic TNBC mouse model [95]. EndoTAG-1 is a PTX-loaded liposome formulation for targeting the activated tumor endothelial cells has entered Phase III clinical trial after showing promising results in contrast to plain drug in TNBC patients [96].

\section{Dendrimers}

Dendrimers are repeating branched monomers arising radially from the central core, formed by the polymerization reaction. These dendrimers are usually $10-100 \mathrm{~nm}$ in size with an amphiphilic nature having a hydrophobic core and a hydrophilic outer periphery [97]. Due to the polyvalent nature of dendrimers like micelles, gene delivery, active targeting can also be possible by conjugating ligands or imaging compounds. Wang et. al. utilizes a fourth-generation poly(amidoamine) dendrimers conjugated to antisense oligodeoxynucleotides (AODNs) for targeting the vascular endothelial growth factor (VEGF) expressed in TNBC cells. The authors report increase accumulation of dendrimers in TNBC-xenograft mouse model with and a significant reduction in expression of VEGF, in comparison to unconjugated nanoparticles due to prolong circulation [98]. Gene delivery using dendrimers was further displayed by Finlay et al. where a third-generation poly(amidoamine) dendrimer was used to deliver YTZ3-15 that can knockdown the TWIST1 transcription factor and is associated with aggressive behavior, metastasis and cellular migration through epithelial-mesenchymal transition (EMT) in TNBC cells [99]. 


\section{Nanoconjugate}

Nanoconjugates are nanoplatforms that are covalent complexes that can bind to multifunctional groups, providing an opportunity to a conjugate peptide(s) of interest such that cell- or tissue-specific targeting can be achieved [100]. Mittapalli et.al. construct an ultra-small hyaluronic acid-PTX nanoconjugates of 2-3 nm that can target CD44 receptor located on the surface of metastatic cancer. CD44 receptor-mediated endocytosis results in 10 folds increase in cellular uptake and significant improvement in overall survival of animals in a TNBC mouse model of brain metastasized in contrast to the plain drug [101]. The multifunctionality of nanoconjugates was further explored and demonstrated when poly ( $\beta$-L-malic acid) (PLMA) nanoplatforms was formed by conjugation of a monoclonal antibody (2C5), and for active targeting: anti-mouse transferrin receptor (TfR) antibody and oligonucleotides (MASONs). This approach allows active targeting of EGFR-positive TNBC cells, resulting in significant tumor inhibition via the EGFR pathway thus controlling tumor progression [102]. A similar nanoconjugate was constructed utilizing PLMA nanoplatforms and produces significant tumor inhibition with no systemic toxicity in TNBC xenograft mice. This strategy was employed for active targeting of three oligonucleotides, leading to a significant arrest of EGFR and laminin411 which are known to cause tumor growth and angiogenesis [103, 104]. A folate-drug nanoconjugate of folic acid and tubulysin B hydrazide (EC1456) entered clinical trial for many types of aggressive cancer including TNBC subtype [105].

\section{Carbon nanotubes}

Carbon tubes are cylindrical structures made from benzene rings and are insoluble in any solvent and thus can be modified chemically to become water soluble or can be functionalized with multiple groups. This unique, nanoneedle shaped, monolithic structure allows passive diffusion to the lipid layer, can be multi-functionalized and can be considered an important drug carrier for cancer therapeutics [106, 107]. Carbon nanotubes single-walled (around 1-2 nm, one layer) or multi-walled (around 5-100 nm, multiple layers), depending upon the number of cylindrical layers. Chemical modification of single-walled carbon nanotubes via an ester bond,

yielded a branched polyethylene glycol chain to which PTX was conjugated to the water-soluble carbon nanotubes. This conjugate exhibited 10 times higher tumor uptake than the conventional Taxol $^{\circledR}$ and little toxicity in murine $4 \mathrm{~T} 1$ breast TNBC model. The improved circulation is probably because of the PEGylation that resulted in more suppression in tumor growth due to the enhanced permeation and retention [108]. Another interesting application of these carbon nanotubes is based on photo thermal-induced ablation, where nanotubes promote cell membrane permeabilization and necrosis, eliminating both tumor mass and BC stem cells, suggesting this could be an effective treatment option for tumor resistant and preventing recurrence [109].

\section{Novel Targeting Approaches}

Novel techniques such as active targeting by attaching ligands (such as small molecules, peptides, aptamers, and antibodies) on nanoparticles to specifically recognize and bind to receptors on target cancer cells, has revolutionized the world of cancer treatment. Such techniques include conjugating the nano-carrier systems to biomolecules including ligands and 
antibodies [110, 111]. Biological cells such as lymphocytes, macrophages, exosomes, erythrocytes, platelets are used as whole-cell carriers to carry the drug-loaded nanoparticles and execute better tumor targeting and potentially destroying the cancer cells (Figure 1-2).

Such strategies would also efficiently minimize off-targeting problems, maximize the therapeutic window and would ensure maximum survival rate and improve the quality of the patient's life. The novel mechanism shows enhanced specificity for cancer cells with minimal side-effects, increasing the therapeutic efficacy up to 100 -fold against drug-resistant cancer stem cells [111]. The reappearance of cancer after a post-treatment and disease-free period is the result of inappropriate drug targeting and low cancer selectivity $[112,113]$. Thus, the application of nano-carriers for active targeting in cancer drug delivery will exponentially alleviate the nonspecific accumulation offering enhancement of therapeutic efficiency [110, 114]. The distinguished physical and chemical properties of nanoparticles make them the appropriate drug delivery carriers. These include their rigidity, hydrophobicity, size, and charge, that facilitates their penetration into biological barriers and effectively deliver the drug at tumor sites [115-117]. These approaches have allowed alteration in the pharmacokinetics and biodistribution of the drug [116]. It is now possible to use chemotherapeutic drugs to specifically target tumor cells by conjugating them with nanoparticles. The previous issues of poor solubility and low bioavailability of the conventional anticancer drugs have been overcome by nano-formulating them [118]. They can not only conjugate the required targeting therapeutic agents but can also deliver it without compromising its activity [119]. This makes nanotechnology one of the greatest man-made wonders of recent times. It has changed the face of diagnosis and treatment of fatal diseases.

\section{Hematopoietic Stem Cells as Carrier for Targeted Delivery}

The remarkable characteristics of hematopoietic stem cells give rise to other blood cells. It makes them an appropriate fit for an efficient drug delivery system in nanomedicine. These blood cells can be broadly divided into three main categories: Erythrocytes (RBCs), Leukocytes (WBCs), and Thrombocytes (platelets). These hemocytes play an important role in the defense mechanism of the body against a wide range of pathogens and foreign particles. Drug delivery systems should achieve maximum therapeutic efficacy and minimal side effects to be considered as potential treatment modalities. Thus, the biological drug delivery system (using blood cells as drug carriers) ensures the biocompatibility and biodegradability of the nanoparticle-loaded cell carriers. The biochemical characteristics favor the non-immunogenicity of blood cells guaranteeing successful drug delivery at target sites [120]. Thus blood cells serve as the natural biological carriers that can bypass the immune surveillance and efficiently deliver the drugs to target cells [121]. Some of the hematopoietic stem cells based nanocarriers that have shown clinical potential in various cancer model has been summarized in Table 1-2.

\section{Erythrocytes}

Erythrocytes or red blood cells (RBCs) are the ideal biological carriers for nanomedicine that can operate without inducing immunological response and provide numerous advantages such as long circulation time, abundant surface ligands, and flexible morphology. RBCs are the 


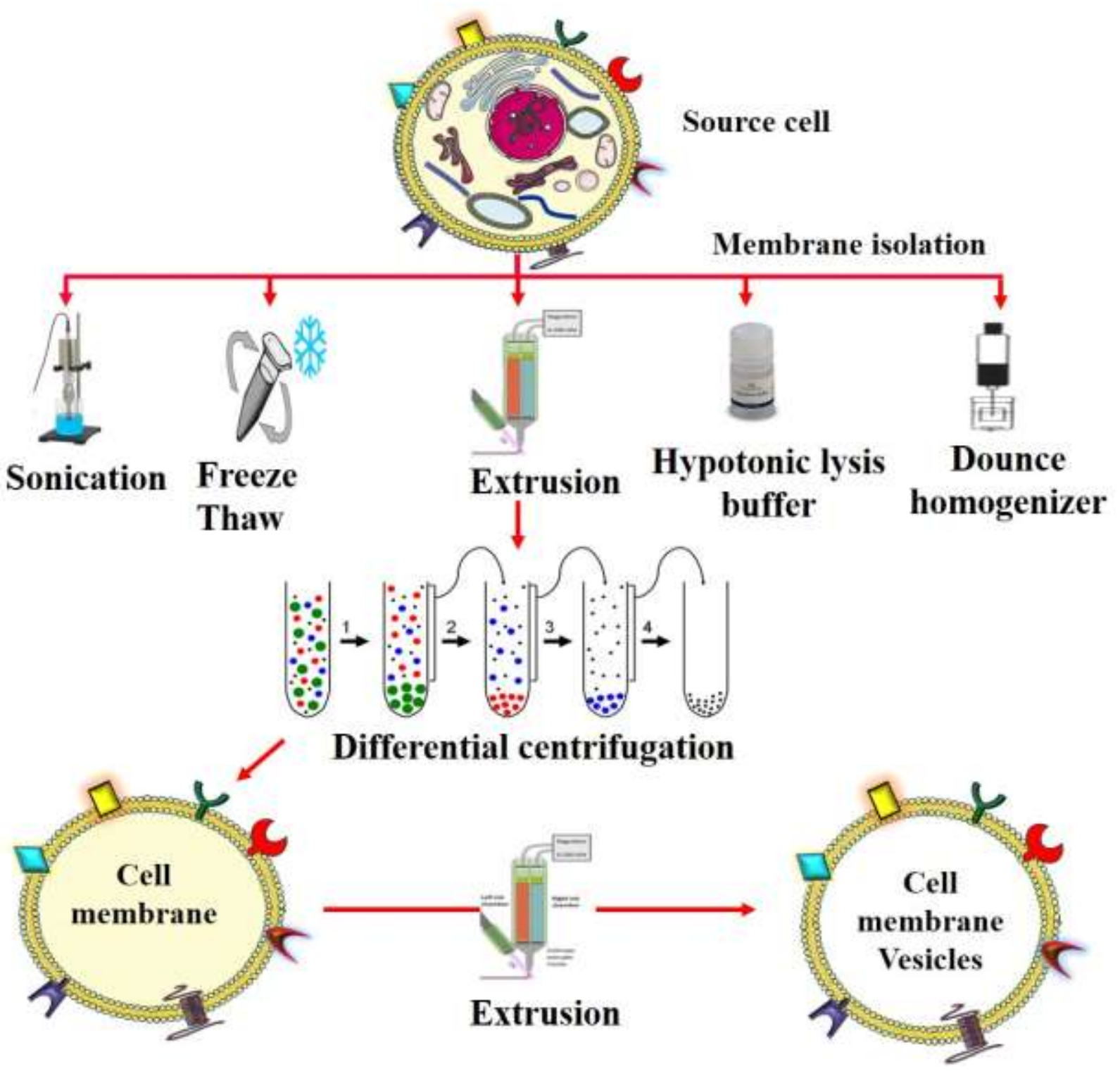

Figure 1-2. Schematic illustration of the preparation of cell membrane coated nanoparticles

An array of various techniques that could be employed to isolate membranes isolated from the source cells by different methods: sonication, freeze-thaw method, extrusion by differential centrifugation, hypotonic lysis buffer and/or Dounce homogenization to finally generate the empty cell membrane vesicles. 
Table 1-2. PTX-based cell membrane coated nanoparticles for therapeutic efficiency/ targeting cancer

\begin{tabular}{|c|c|c|c|c|}
\hline $\begin{array}{l}\text { Cell } \\
\text { type }\end{array}$ & $\begin{array}{l}\text { Nanoparticle } \\
\text { core }\end{array}$ & $\begin{array}{l}\text { Intended } \\
\text { use }\end{array}$ & $\begin{array}{c}\text { Targeted cancer type and benefits } \\
\text { achieved }\end{array}$ & Ref. \\
\hline $\begin{array}{l}\text { Lymphocytes } \\
\text { membrane }\end{array}$ & $\begin{array}{l}\text { Membrane coated on } \\
\text { PLGA nanoparticles, } \\
\text { low-dose irradiation. }\end{array}$ & $\begin{array}{l}\text { Subcutaneous } \\
\text { model using: } \\
\text { MKN45 cells. }\end{array}$ & $\begin{array}{l}\text { Nanoparticles caused } 56.68 \% \text { tumor } \\
\text { inhibition, liver accumulation in } 96 \text { hours } \\
\text { while LDI caused more tumor } \\
\text { accumulation with } \sim 89 \% \text { tumor inhibition. }\end{array}$ & [122] \\
\hline Monocytes & $\begin{array}{l}\text { pH-responsive } \\
\text { amphiphilic } \\
\text { copolymer, } \\
\text { polyethylene glycol- } \\
\text { block-poly[(1,4- } \\
\text { butanediol)- } \\
\text { diacrylate- } \beta-\mathrm{N}, \mathrm{N}- \\
\text { diisopropylethyl } \\
\text {-enediamine] (PDB) } \\
\text { and phagocyting this } \\
\text { in Ly6C mi monocyte } \\
\text { isolated from } \\
\text { peripheral blood. }\end{array}$ & $\begin{array}{l}\text { Metastatic } \\
4 \mathrm{~T} 1 \mathrm{BC} \text {. }\end{array}$ & $\begin{array}{l}2 \text { folds increase in tumor accumulation } \\
\text { was observed with the monocyte loaded } \\
\text { nanoparticles, with relatively less } \\
\text { nonspecific uptake in lung and liver, } \\
\text { compared to blank nanoparticles (no } \\
\text { monocytes). Also, highest AUC was } \\
\text { observed } 7.20 \text {-folds higher than PTX, with } \\
\text { highest tumor suppression of } 96.8 \% \text { over } \\
\text { only } 50.4 \% \text { inhibition was attained with } \\
\text { nanoparticles (without monocytes). Also, } \\
\text { lung metastasis decreased by } 99.2 \% \text { with } \\
\text { these nanoparticles, over } 50 \% \text { decrease } \\
\text { with non-monocytes nanoparticles. }\end{array}$ & [123] \\
\hline $\begin{array}{l}\text { Macrophage } \\
\text { membrane }\end{array}$ & $\begin{array}{l}\mathrm{pH} \text { sensitive polymer } \\
\text { cationic 2- } \\
\text { aminoethyldiisopropy } \\
1 \text { with IGF1R } \\
\text { targeting peptide, } \\
\text { after PEGylation and } \\
\text { macrophage } \\
\text { membrane. }\end{array}$ & $\begin{array}{l}\text { orthotopic } \\
\text { BC: MDA- } \\
\text { MB-231 cells. }\end{array}$ & $\begin{array}{l}\text { Significantly tumor accumulation and } \\
\text { towards the center of the tumor was } \\
\text { achieved, relative to group without the } \\
\text { non-pH sensitive polymer and without } \\
\text { macrophage coating, due to membrane } \\
\text { coated tumor homing effect and pH- } \\
\text { sensitive drug release by the polymer. The } \\
\text { peptide also enhanced the fluorescence } \\
\text { intensity due to IGF1R mediated uptake } \\
\text { pathway. }\end{array}$ & [124] \\
\hline $\begin{array}{l}\text { Platelet } \\
\text { membrane }\end{array}$ & $\begin{array}{l}\text { A synthetic peptide } \\
\text { with dendritic } \\
\text { disulfide conjugate of } \\
\text { PTX coupled with } \\
\text { PEG via click } \\
\text { reaction, to yield a } \\
\text { redox-responsive } \\
\text { micelle that could } \\
\text { capture internal } \\
\text { activated platelets }\end{array}$ & $\begin{array}{l}\text { Xenograft } \\
\text { TNBC } \\
\text { tumors: } \\
\text { MDA-MB- } \\
231 \text { cells. }\end{array}$ & $\begin{array}{l}\text { The micelles were recruited to the surface } \\
\text { of the activated platelets, due to } \\
\text { overexpression of P-selectin on the } \\
\text { platelets and adhere to it. } \\
\text { Relative to PTX these targeted micelle } \\
\text { exhibits enhanced targeting to the primary } \\
\text { TNBC tumors as well as lung and liver } \\
\text { metastasis. This is due to the interaction } \\
\text { between activated platelets and ICAM } \\
\text { overexpressed at the metastatic sites, by } \\
\text { tumor cell receptor-targeting strategies. }\end{array}$ & [125] \\
\hline $\begin{array}{l}\text { Platelet } \\
\text { membrane }\end{array}$ & $\begin{array}{l}\text { Nanogel with TNF- } \\
\alpha, \text { RGD peptide } \\
\text { (Nanoparticle1). } \\
\text { Dextran nanoparticles } \\
\text { with coated with } \\
\text { platelet membrane } \\
\text { with PTX } \\
\text { (Nanoparticle2) }\end{array}$ & $\begin{array}{l}\text { TNBC tumor } \\
\text { using MDA- } \\
\text { MB-231 cells. }\end{array}$ & $\begin{array}{l}\text { Nanoparticle } 1 \text { induces tumor vascular } \\
\text { inflammation and RGD peptide caused } \\
\text { significant accumulation in the tumor by } 5 \\
\text { folds, relative to nanoparticles without } \\
\text { RGD. Whereas nanoparticle } 2 \text { cause greater } \\
\text { tumor accumulation by } 5 \text { folds relative to } \\
\text { the nanoparticles without coating. }\end{array}$ & [126] \\
\hline
\end{tabular}


Table 1-2. (Continued)

\begin{tabular}{|c|c|c|c|c|}
\hline $\begin{array}{l}\text { Cell } \\
\text { type }\end{array}$ & $\begin{array}{c}\text { Nanoparticle } \\
\text { core }\end{array}$ & $\begin{array}{l}\text { Intended } \\
\text { use }\end{array}$ & $\begin{array}{c}\text { Targeted cancer type and benefits } \\
\text { achieved }\end{array}$ & Ref. \\
\hline $\begin{array}{l}\text { RBC } \\
\text { membrane }\end{array}$ & $\begin{array}{l}\text { Thermo-responsive } \\
\text { hybrid nanoparticle } \\
\text { composed of } \\
\text { poly(caprolactone)- } \\
\text { ester endcap polymer } \\
\text { (PCL), } \\
\text { dipalmitoylphosphati } \\
\text { dylcholine (DPPC) } \\
\text { poloxamer } 188 \text { and } \\
\text { membrane coating. }\end{array}$ & $\begin{array}{l}4 \mathrm{~T} 1 \\
\text { orthotropic } \\
\text { tumor } \\
\text { mimicking } \\
\text { metastasis BC. }\end{array}$ & $\begin{array}{l}\text { A } 12.3,2.6 \text { - and 3-folds increase of } \\
\text { fluorescent dye (DiR) at the tumors, liver } \\
\text { and lung metastasized sites, respectively in } \\
\text { comparison to free DiR. } 69.2 \% \text { and } 12.6 \% \\
\text { tumor inhibition were achieved by the } \\
\text { nanoparticles, PTX respectively, in } \\
\text { comparison to control. Also, } 98.6 \% \text { lung } \\
\text { metastasis was achieved. }\end{array}$ & [127] \\
\hline $\begin{array}{l}\text { RBC } \\
\text { membrane }\end{array}$ & $\begin{array}{l}\text { PCL, poloxamer } 188 \text {, } \\
\text { co-administrated with } \\
\text { the tumor penetrating } \\
\text { peptide, iRGD and } \\
\text { membrane coating. }\end{array}$ & $\begin{array}{l}\text { Metastatic } \\
\text { 4T1 breast } \\
\text { tumor model. }\end{array}$ & $\begin{array}{l}\text { The half time of the cell membrane } \\
\text { nanoparticles was } 32.8 \text { hours ( } 5.8 \text { and } 16.9 \\
\text { folds higher than that of polymeric } \\
\text { nanoparticles and Taxol, respectively). } \\
\text { These nanoparticles in combination with } \\
\text { iRGD yield } 2.89,3.02 \text { folds higher tumor } \\
\text { fluorescence uptake, } 90 \% \text { tumor growth } \\
\text { inhibition and } 94.8 \% \text { lung metastasis were } \\
\text { achieved. All comparisons were with } \\
\text { uncoated nanoparticles with iRGD and cell } \\
\text { membrane nanoparticles (without iRGD). }\end{array}$ & [128] \\
\hline $\begin{array}{l}\mathrm{RBC} \\
\text { membrane }\end{array}$ & $\begin{array}{l}\text { Ferric oxide }\left(\mathrm{Fe}_{3} \mathrm{O}_{4}\right) \\
\text { and } \mathrm{O}-\text { carboxymethyl } \\
\text { chitosan }(\mathrm{CMC}) \\
\text { nanoparticles co- } \\
\text { encapsulated PTX } \\
\text { and doxorubicin with } \\
\text { Arg-Gly-Asp (RGD) } \\
\text { and membrane } \\
\text { coating. }\end{array}$ & $\begin{array}{l}\text { Subcutaneous } \\
\text { xenograft } \\
\text { model of lung } \\
\text { carcinoma. }\end{array}$ & $\begin{array}{l}\text { Synergistic effect of RBC membrane } \\
\text { magnetic nanoparticles and RGD ligand, } \\
\text { on the application of magnetic field, } \\
\text { increased fluorescent uptake at the excised } \\
\text { tumors by } \sim 17 \text { times and significant tumor } \\
\text { reduction in contrast to naked } \\
\text { nanoparticles (without membrane coating } \\
\text { and RGD). }\end{array}$ & [129] \\
\hline $\begin{array}{l}\mathrm{RBC} \\
\text { membrane }\end{array}$ & $\begin{array}{l}\text { DSPE-PEG-MAL } \\
\text { coupled with tumor- } \\
\text { penetrating bispecific } \\
\text { recombinant protein } \\
\text { (anti-EGFR-iRGD) } \\
\text { with RBC membrane } \\
\text { coating. }\end{array}$ & $\begin{array}{l}\text { Subcutaneous } \\
\text { tumors of } \\
\text { gastric cancer } \\
\text { cells: MKN45. }\end{array}$ & $\begin{array}{l}\text { RBC membrane coating prolonged } \\
\text { nanoparticle circulation in the tumors from } \\
2-48 \text { hours. The synergistic effect of anti- } \\
\text { EGFR-iRGD along with membrane coated } \\
\text { nanoparticles caused tumor inhibition by } \\
61 \% \text { in contrast to only } 21 \% \text { inhibition } \\
\text { with the PTX, relative to the control. }\end{array}$ & {$[130]$} \\
\hline $\begin{array}{l}\mathrm{RBC} \\
\text { membrane }\end{array}$ & $\begin{array}{l}\text { pH sensitive poly (L- } \\
\gamma- \\
\text { glutamylcarbocistein) } \\
\text {-nanoparticles } \\
\text { wrapped with RBC } \\
\text { membrane. }\end{array}$ & $\begin{array}{l}\text { Subcutaneous } \\
\text { tumors of lung } \\
\text { cancer cells } \\
\text { NCI-H } 460 .\end{array}$ & $\begin{array}{l}\text { AUC for } 72 \text { hours was } 2.44 \text { folds higher } \\
\text { with RBC coating nanoparticles, relative to } \\
\text { non-coated nanoparticles ( } 2082 \text { and } 850.2 \\
\text { respectively). Similarly, stronger tumor } \\
\text { inhibition was observed. }\end{array}$ & [131] \\
\hline
\end{tabular}


Table 1-2. (Continued)

\begin{tabular}{|c|c|c|c|c|}
\hline $\begin{array}{l}\text { Cell } \\
\text { type }\end{array}$ & $\begin{array}{l}\text { Nanoparticle } \\
\text { core }\end{array}$ & $\begin{array}{l}\text { Intended } \\
\text { use }\end{array}$ & $\begin{array}{c}\text { Targeted cancer type and benefits } \\
\text { achieved }\end{array}$ & Ref. \\
\hline $\begin{array}{l}\mathrm{RBC} \\
\text { membrane }\end{array}$ & $\begin{array}{l}\text { RBC membrane } \\
\text { coated nanoparticles. }\end{array}$ & $\begin{array}{l}\text { Mammalian } \\
\text { intestinal } \\
\text { mucosal cells } \\
\text { (MDCK- } \\
\text { MDR1) }\end{array}$ & $\begin{array}{l}\text { Permeability was enhanced by } 3.5-\& 16.2 \\
\text { folds than free PTX in MDCK-MDR } 1 \text { cell } \\
\text { monolayers and intestinal mucosa, } \\
\text { respectively. The presence of the RBC } \\
\text { membrane prolonged the circulation time } \\
\text { by increasing the mean residence time of } \\
\text { the nanoparticles by } 1.81 \text { folds, AUC by } \\
14.2 \text { folds and } C_{\max } \text { by } 6 \text { folds, relative to } \\
\text { free PTX. }\end{array}$ & [132] \\
\hline Neutrophil & $\begin{array}{l}\text { CXCL1 chemokine } \\
\text { laden thermosensitive } \\
\text { hydrogel of PLGA- } \\
\text { PEG-PLGA } \\
\text { nanoparticles, } \\
\text { encapsulated by the } \\
\text { endogenous } \\
\text { neutrophils. }\end{array}$ & $\begin{array}{l}\text { B16F10 } \\
\text { murine } \\
\text { melanoma } \\
\text { cells. }\end{array}$ & $\begin{array}{l}\text { Neutrophils sequester the nanoparticles } \\
\text { and in } 8 \text { hours shows } 82.2 \% \text { uptake } \\
\text { implying viability of neutrophils were not } \\
\text { affected by the PTX loaded nanoparticles. } \\
\text { Fluorescent dye (DiD)increased } \\
\text { significantly at the tumor site with CXCL1 } \\
\text { from 1-8hours, unlike without the CXCL1 } \\
\text { group, suggesting the presence of the } \\
\text { chemokine was primarily causing } \\
\text { recruitment of the neutrophil loaded } \\
\text { nanoparticles. Synergistic effect of } \\
\text { CXCL1 and neutrophil loaded } \\
\text { nanoparticles caused the most tumor } \\
\text { inhibition of } 67.28 \%, 2.13 \text { folds higher } \\
\text { without the CXCL1 group ( } 46.95 \%) \text {. }\end{array}$ & [133] \\
\hline Neutrophil & $\begin{array}{l}\text { Cationic liposomes } \\
\text { made of } 1,5- \\
\text { dioctadecyl-N- } \\
\text { histidyl-L-glutamate } \\
\text { (HG2C18), } \\
\text { internalized in mouse } \\
\text { bone marrow derived } \\
\text { neutrophils. }\end{array}$ & $\begin{array}{l}\text { G422 } \\
\text { glioblastoma } \\
\text { cells. }\end{array}$ & $\begin{array}{l}\text { Highest fluorescent intensity of DiR dye } \\
\text { was observed with neutrophil } \\
\text { nanoparticles in the tumor region of the } \\
\text { brain collected from surgically treated } \\
\text { glioma tumors. The nanoparticles migrated } \\
\text { to the infiltrating glioma cells GFP-G422 } \\
\text { cells, up to } 96 \text { hours, suggesting enhanced } \\
\text { targeting due to neutrophil which causes } \\
\text { inflammatory response after surgery. } \\
\text { AUC brain was the highest suggesting } \\
\text { highest targeting efficiency due to } \\
\text { neutrophils. }\end{array}$ & [134] \\
\hline Neutrophil & $\begin{array}{l}\text { Commercially } \\
\text { available PTX } \\
\text { formulation: } \\
\text { Abraxane dispersed } \\
\text { within human NEs in } \\
\text { combination with } \\
\text { radiotherapy by } 5 \text { - } \\
\text { Gy. }\end{array}$ & $\begin{array}{l}\text { Gastric } \\
\text { cancer: } \\
\text { SNU719 } \\
\text { tumor-bearing } \\
\text { mice. }\end{array}$ & $\begin{array}{l}\text { Tumor reduction was maximum when } \\
\text { radiotherapy and neutrophil nanoparticles } \\
\text { were combined. The radiation disrupts the } \\
\text { tumor and allows the neutrophils to be } \\
\text { homed at the tumor site, due to the release } \\
\text { of inflammatory cytokines. Radiation } \\
\text { therapy with only neutrophil (no } \\
\text { nanoparticle) did not produce any } \\
\text { significant anti-tumor effect. }\end{array}$ & [135] \\
\hline
\end{tabular}


most abundant cells in the blood, comprising almost a quarter of the total volume, performing the crucial role of transporting oxygen and carbon dioxide throughout the body. Its unique physiological characteristics make them natural and versatile carriers and are applied to enhance the specificity of therapeutics and to alleviate the negative effects through the controlled delivery of targeting and releasing agents. These biconcave discoid cells have a diameter of $7 \mu \mathrm{m}$ which is responsible for its high drug loading capacity. Upon maturation, RBCs lose their nucleus and other organelles to increase its surface area manifold. Being enucleate, RBCs become suitable carriers for drugs that are targeted to the nuclei of diseased cells. Their half-life of over 100 days, distinguished macromolecular structures portrays their potential as drug delivery systems. The presence of carbohydrates, proteins, and phospholipids on the outer member layer provides stability and deformability allowing cells to target extremely small vascular structures. [136]. The erythrocyte membrane simultaneously undergoes reversible changes between expanded and tight networks to maintain its structural integrity. This process of avoiding intravascular hemolysis is governed by the cytoskeleton structures of the RBC membrane [137]. The novel drug delivery system involves the transient, controlled opening of the RBC membrane, the encapsulation of the nano-carrier loaded anti-cancer drugs, and the subsequent resealing of the outer membrane.

The application of erythrocytes as drug delivery vehicles in cancer treatment has been extensively investigated because of its main biochemical and physiological propensities: minimal toxicity, high drug loading capacity, slow molecular release, biocompatibility, and reduction of immune reaction ions. It encapsulates anti-cancer drugs such as daunorubicin for leukemia treatment, 5-fluorouracil (methotrexate) [123], and 1-Asparaginase (1-ASNase) [86]. Erythrocytes encapsulate and protect 1-ASNase from degradation and reduce the severity of side effects and exhibits longer half-life. Thus, increasing the efficiency ten-fold than native l-ASNase in the treatment of asparagine synthetase-deficient acute myeloid lymphoblastic leukemia. Here, asparaginase-loaded RBCs serve as 'bio-reactors' to deplete asparagine, an amino acid required for cancer cell growth, from the blood with a tendency of low coagulation disorders and minimum allergic reactions [124].

Methotrexate (MTX) is an antimetabolite and antifolate agent used in solid tumors and hematological diseases. MTX-loaded erythrocyte treatment increases the average survival time of mice bearing hepatoma ascites tumors by $28.5-42.8 \%$ than native MTX. This implies the use of RBCs as a drug delivery system for chemotherapeutic agents, especially in vitro and in vivo use of antineoplastic agents [138]. The application of whole cells RBC in drug delivery is myriad, even ranging from loading $\mathrm{RBC}$ with $\mathrm{Au}$ nanoparticles and are exposed to a nearinfrared laser, the permeability of the membrane increases which allows site-specific drug release. Magnetic targeting using an external magnetic field was applied to doxorubicin-loaded RBCs which are bound to iron-oxide nanoparticles pre-coated with a photosensitizer in the chemotherapy of cancer [139]. The modification of the pharmacokinetic and pharmacodynamics parameters of the drug by carrier erythrocytes maintains a relatively inert intracellular environment, decreased fluctuations in concentration, reduces drug side effects. Thus, the molecular mechanisms of erythrocytes make them perfect candidates as carriers of convention and new anti-cancer drugs [137]. 


\section{Leukocytes}

White blood cells (WBCs) or leukocytes are an integral part of the innate immune system of the body in response to pathogen invasion. When tissue damage, bacterial and viral infections occur, leukocytes are deployed in circulation to combat those changes. Therefore, inflammation can be defined as a defense mechanism of the body to fight foreign invaders (physical, chemical, antigens) and maintain homeostasis [140]. Owing to their aiming movement and transmigration ability, leukocytes are specifically targeted to deliver nanotherapeutics into diseased tissues. The pathogenesis of most types of cancers is in correlation to uncontrolled inflammation. Thus, hijacking leukocytes and deploying them as delivery vehicles to transport anti-cancer therapeutics across blood vessel barriers directly to the tumor microenvironment [78-80].

\section{Neutrophils}

Neutrophils are first-line defenders arriving at the inflammatory site migrating across the endothelial layer, known as the neutrophil transmigration, to fight pathogens and initiate a phase of repair. They are the most abundant white blood cells (50-70\%) in humans and are thus excellent candidates as carriers for targeted drug delivery. Neutrophils are associated with tumor progression as well as tumor inhibition. These neutrophils that infiltrate tumor sites are called tumor-associated neutrophils (TANs) [141]. The recruitment of TANs is mediated by their surface protein composition and chemokine activity. They are phenotypically distinct from normal circulating neutrophils. They can be of two types: Pro-tumorigenic (N2) TANs (produced in the tumor microenvironment) and anti-tumorigenic (N1) TANs (ability to kill tumor cells). Targeting these neutrophils would allow better specificity and enhanced therapeutic efficacy of anti-cancer nanotherapeutics [96].

IL-8 is associated with numerous pro-tumor pathways, thus serves as an excellent target for treatment via blocking its function. Preclinical tests involving IL-8 antagonists have shown reduced tumor growth, angiogenesis, metastasis, and increased tumor cell apoptosis [84-87]. Studies have shown that inhibition of BM and ECM breakdowns by TANs derived NE and MMP-9 can dramatically reduce tumor angiogenesis and lung adenocarcinoma in a murine model [88]. In another study, the successful downregulation of a murine model tumor ICAM-1 expression via shRNA reduced colorectal adenocarcinoma by $45 \%$. In cancer treatments, where surgical resection has negative outcomes, anti-TAN therapy serves a potential approach with higher disease-free survival rates [89,90]. In a recent study, Chu et al demonstrated gold nanorods (GNRs) linked with anti-CD11b Abs were able to exponentially decrease tumor growth and increased survival rates in a lung carcinoma model of mice. Photothermal therapy was undertaken as the therapeutic method because GNRs can absorb infrared light to generate local heat to destroy the tumor [91]. In one of their previous studies, they combined TA99 monoclonal antibodies and albumin nanoparticles to treat melanoma in a mouse model. This was achieved through the mechanism of antibody-dependent cellular cytotoxicity (ADCC), wherein the albumin NPs were loaded with photodynamic therapy (PDT) agents which activated the infiltration of neutrophils into tumor sites markedly reducing tumor growth and thus emerging as a novel strategy for immunotherapy in cancer treatment [92]. Neutrophils have been heavily employed as immune cell carriers for delivering nanoparticles to inflammation sites. For example, piceatannol-loaded and TCPA-1/cefoperazone acid-loaded albumin nanoparticles with 
neutrophils as delivery vehicles are used as treatment approaches for acute lung injury and pyropheophorbide-a loaded albumin nanoparticles are targeted against melanoma [80].

Therefore, neutrophil-mediated delivery of nanotherapeutics has immense potential to dramatically increase target specificity, therapeutic efficacy, and provide a translational effect. Their intrinsic properties of transmigration, ability to infiltrate in huge numbers in response to inflammation, and their first responder nature makes them appropriate biological carriers to deliver nanotherapeutics [80].

\section{Monocytes/Macrophages}

Monocytes are an integral part of immune-oncology with the unique characteristic to be able to differentiate into tissue macrophage, known as tumor-associated macrophages (TAMs), after crossing the endothelial barrier. The ability of TAMs to reach the hypoxic areas of tumor microenvironment makes them excellent targets for a biometric delivery system (BDS). TAMs constitute $70 \%$ of the cell mass in breast carcinoma and can be classified into M1 and M2 types. M2-like macrophages are responsible for tumor growth and progression whereas M1 phenotype is associated with killing tumor cells. Owing to their innate phagocytotic capability, monocytes can be loaded with a variety of nanotherapeutics and serve as "Trojan Horse" delivery vehicles reaching otherwise inaccessible tumor regions. Once these cells reach the tumor sites, they differentiate into macrophages and their nanoparticle-based therapeutic function could be initiated by near-infrared (NIR) illumination, henceforth destroying the TAMs associated with tumor metastasis [80, 93-96]. In a recent study, Choi et al demonstrated that the tumor's natural recruitment of monocytes may be exploited for nanoparticle-based drug delivery and therapeutics. To avoid any harm to surrounding cellular entities of the host, the drug was loaded in Au nanoshells, nanoparticles consisting of a silica core surrounded by a thin Au shell. Human breast tumor spheroids (T47D) were utilized as a model to examine the therapeutic efficacy and cellular uptake of the Au nanoshells. Photothermal therapy was used and it was observed that tumor cell death resulted in incident laser powers above a certain threshold. They successfully demonstrated the potential of monocytes as delivery vehicles into hypoxic tumors and established a foundation for a novel drug delivery system [97].

Poor efficiency of conventional drug delivery systems into the sites of metastases leads to high mortality rates of BC. In a study about anti-metastasis therapy, He et al demonstrated that loading legumain-activated nanoparticles into inflammatory monocytes can actively target lung metastases of $\mathrm{BC}$ and inhibit tumor progression. The cytotoxic anti-cancer drug of mertansine is conjugated to poly (styrene-co-maleic anhydride) with a legumain-sensitive peptide and selfassembled into nanoparticles (SMNs) and then loaded into Ly6c+ inflammatory monocytes to prepare the SMNs loaded monocytes delivery system (M-SMNs). In this biometric delivery system, the SMNs would remain inactive until they come in contact with the metastatic niche. This prevents early drug release and ensures the living state of monocytes which is required for the efficient anti-metastatic effect. Upon reaching the tumor microenvironment, the monocytes differentiate into macrophages to release the anti-cancer drug as free drug molecules by destroying the macrophages. The study shows plausible evidence of inhibition of the proliferation, migration, and invasion activities of metastatic 4T1 BC cells [98]. 
In another study by $\mathrm{Fu}$ et. al., a biometric delivery system (BDS) was established using a mouse macrophage-like cell line to demonstrate the anti-cancer efficacy of lung metastasis of BC (4T1 cells). A mouse macrophage-like cell line (RAW265.7) with similar functions to primary macrophage cells were used as delivery vehicles and loaded with doxorubicin (DOX) to serve as an anti-cancer BDS. The DOX loaded macrophage system showed tumor suppression, metastasis inhibition, with an increased life span of the host and reduced toxicity to other healthy tissues and organs as compared to their control groups. Thus, DOX encapsulating macrophages proved to be an efficient delivery system into tumor sites and showed an enhanced therapeutic effect by inhibiting tumor growth to a great extent [142]. Exploiting autologous macrophages $(\mathrm{M} \Phi)$ for anti-cancer therapy has been developed in the late $20^{\text {th }}$ century and it still has unexplored areas to venture upon. Tumors, however, promote normal MФ functions of tissue repair, resulting in tumor growth, over inflammatory responses. in the tumor microenvironment, the replacement of $M \Phi$ with genetically engineered monocytes or drug-loaded monocytes can be potential delivery vehicles for nanotherapeutics. The encapsulation of drugs in $M \Phi$ has an added advantage of an extended half-life, protected from clearance by the endogenous RES system. The multi-faceted benefits of utilizing $\mathrm{M} \Phi$ as delivery vehicles have been demonstrated in a study by Escobar et al. They used a spontaneous BC mouse model (MMTV-PyMT), wherein they performed hematopoietic stem cell transplants with selective expression of IFN $\alpha$ in TIE2+ tumor-associated MФs in the model. The highly localized TIE2+-MФ-mediated delivery of IFN $\alpha$ reduced lung metastatic areas 5-fold and primary tumor size 3-fold without apparent toxic effects to the host organism. The plasticity and versatility of autologous macrophages make them ideal candidates for novel drug delivery systems to enhance the specificity of cell therapeutics [100-102].

\section{Thrombocytes}

Platelets are anucleated, small subcellular fragments of megakaryocytes with a half-life of 7-10 days that circulate in the bloodstream and are activated during a vascular endothelial dysfunction or damage [103]. They are associated with inflammatory cells and play a central role in the cancer microenvironment by cell-cell communication and ability to uptake a plethora of different molecules [104-106]. Activated platelets are key contributors to tumorigenesis, metastasis, tumor growth, and angiogenesis. B-thromboglobin and P-selectin, markers of platelet activation, are abundant in patients with $\mathrm{BC}$, suggesting tumor cell-induced platelet aggregation (TCIPA) [107]. Once activated, these platelets facilitate cancer cell survival and their adhesion to the endothelium. Thus, tumor-associated platelets can be potential therapeutic targets and serve as effective delivery vehicles in anti-cancer treatment modalities [107,108].

Targeting specific platelet receptors and tumor-associated platelets has become an emergent field of delivery of anti-tumor therapeutics. Zhang et. al. designed a biocompatible liposomal nanoparticle with a tumor-homing peptide on the surface and loaded with the reversible platelet inhibitor ticagrelor, known as CREKA-Lipo-T, to demonstrate its ability to block tumor cell acquisition of an invasive phenotype and tumor cell adhesion of platelets. They showed target specificity and therapeutic efficiency of CREKA-Lipo-T with respect to 4T1 solid tumors and established a promising nanoparticle drug delivery system for anti-cancer therapy [109]. In another study by the same group, they constructed a polymer-lipid-peptide-based drug delivery system (comprised of matrix metalloproteinase 2 (MMP2)-cleavable peptides, lecithins,

and PEGylated phospholipids to form an enzyme-responsive drug release known as PLP-D-R) to 
co-deliver a platelet-depleting antibody (R300) and chemotherapeutic drug (Dox). They successfully demonstrated the anti-cancer efficacy of PLP-D-R in an MCF7 tumor-bearing nude mouse model that showed enhanced tumor suppression with minimal bleeding complications. Moreover, they also showed enhanced nanoparticle retention (almost thrice as compared to control groups), tumor regression, and metastasis inhibition in tumor-associated platelet depleted models of mice [110,111].

Novel strategies involving anti-platelet therapeutics are gathering momentum and emerging as an alternative approach to conventional anti-cancer modalities. In a recent study, Guillem-Llobat et. al. demonstrated that the administration of low-dose aspirin, a member of nonsteroidal anti-inflammatory drugs (NSAIDs) which inhibits prostanoid biosynthesis by inhibiting the action of COX-1 and COX-2, reduced the formation of lung metastases [112]. In another study by Wang et. al., it was shown that in P2Y12-deficient mice, which is an ADP platelet receptor, the co-administration of the drug clopidogrel with aspirin attenuates the development of hepatocarcinoma and improves survival rates of the host [113]. In a murine model of lung cancer, Nierodzik et. al. demonstrated how blocking the platelet GPIIb/IIIa receptor, using the monoclonal antibody 10E5, prevented the cells from metastasizing [114]. In another study about BC treatment, the curative effect of heparin and fondaparinux against tumor cells was demonstrated. They achieved this by indirect inhibition of thrombin and Factor Xa, thereby inhibiting the activation of platelets by BC cells [115]. A study exploiting the utilization of platelet interactions with pro-metastatic BC cells to develop a platelet-inspired nano vehicle system for targeted delivery of the anti-cancer drug DOX. Pan et al designed a liposomal nano construct that can bind simultaneously to GPIIb-IIa like integrins and P-selectin on the highmetastatic MDA-MB-231 human BC cells. They successfully achieved their goal of killing the tumor cells and enhanced the therapeutic efficiency by their platelet-inspired metastasis-targeted drug delivery approach [116]. All the above studies establish the potential of utilizing platelets as delivery vehicles and the promising future of platelet-inspired anti-cancer therapeutics.

\section{Cell-Membrane Coated Nanoparticles for Targeted Delivery}

Cell membranes isolated from the parent cell are subjected to a continuous process, to isolate the various cellular components. Cells are previously treated with hypotonic buffer under the protection of protease inhibitor are followed by series of ultracentrifugation. This enables the removal of cell contents, including enzymes, nucleus and other cellular components. The membranes are then coated on the surface of the nanoparticle core, via extrusion, sonication and/or electroporation techniques (Figure 1-3). The membranes impart a double layer, owing to the structure of the lipid bilayer and are about 50-800 $\mathrm{nm}$ in size $[143,144]$. The transmembrane proteins and all the relevant membrane-bound antigen required for imparting a biological characteristic are preserved on the cell membrane, with no loss in functionality during or after translocating the membrane onto the surface of the nanoparticles [145]. This allows the nanoparticles to be camouflaged by the cell membranes, preventing degradation by the patient's immune system [146]. The cell membrane coated nanoparticles can interact with cells of the targeted site, due to the presence of receptors, originated from the derived parent cells that make them able to deliver therapeutics at these targeted sites [147]. Thus these coated nanoparticles

offer advantages in contrast to plain nanoparticles, such as i) prolonged circulation [148] 


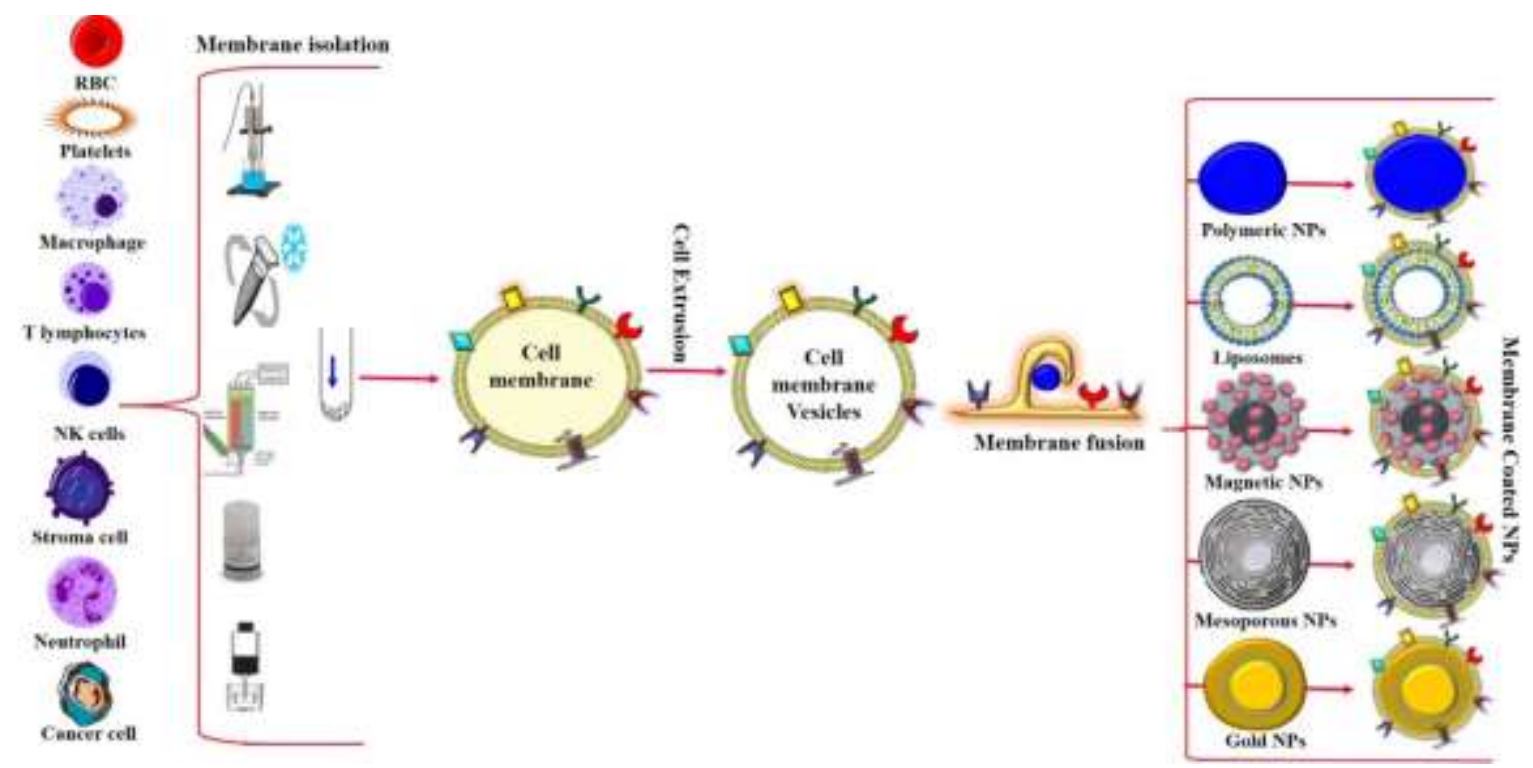

Figure 1-3. Strategies to coat the surface of nanoparticles with cell membrane coating Different types of source cells are employed to generate empty cell membrane nanovesicles that are extracted from either Erythrocytes/RBC, Leukocytes: neutrophils, monocytes/macrophages, T-lymphocytes, Thrombocytes/platelets, Mesenchymal stem cells, Cancer cells. These membrane-derived vesicles retain the surface protein, antigens from the source cells when coated on the surface of nanoparticles, can directly target or bind to the target site of cancer. 
ii) cell targeting [149], iii) circumventing the immune system clearance [150], iv) detoxification [151], v) mediating intracellular communications via endocytosis, which can be used to deliver microRNA, mRNA [152, 153]. However, scalability, production, isolation of these membranes for a bulk manufacturing process needs to be addressed for better clinical applications.

\section{Erythrocyte membrane-coated nanoparticles}

Erythrocytes are the most common cell component with a unique biconcave discoidal shape of approximately 7-8 $\mu \mathrm{m}$ diameter, allowing a large volume of therapeutic cargo to be loaded. They bear high mechanical flexibility allowing them to be able to squeeze through very small blood capillaries even when maintaining the constant surface area. It can circulate for approximately 120 days and eventually clearance by the RES [154]. Additionally, they prevent unwanted macrophage uptake, provides specificity to the target, extend biocompatibility, biodegradability and non-immunogenic nature, limits activation of other competitor cellular components [155]. Erythrocytes membrane coated nanoparticles are prepared by extracting the membrane from the cellular components, yet preserving their original protein antigens, that provides the innate natural targeting ability. Researchers have utilized hypotonic solution to extract the cellular contents and used the membrane to coat PLGA polymer nanoparticles and demonstrated the prolonged circulation of 72 hours over non coated PLGA nanoparticles [148] and suppress 98\% lung metastasis in metastatic BC model [127]. Su et al. demonstrated similar results, erythrocytes membrane coated PLGA nanoparticles prolong circulation due to protein receptors present on the surface of these membranes and also in combination with integrating iRGD that provide specific targeting of metastatic breast tumor model. The authors show inhibition of more than $90 \%$ tumor growth and $95 \%$ of the lung metastasis with these nanoparticles as they can escape clearance by RES and circulate longer.

Additionally, the presence of tumor penetrating peptide iRGD serves as a receptor for lung metastatic sites that overexpress $\alpha v \beta 3$ integrin and neuropilin-1, which allows the nanoparticle to specifically target and penetrate tumors. The authors also report enhanced retention of membrane coated nanoparticles at the metastatic regions, without the iRGD peptide, indicating the innate tendency of the erythrocytes membrane proteins to accumulate near the metastatic and tumor region [128]. Guo et. al. developed a nano vaccine utilizing the ability of erythrocytes coated nanoparticles to target antigen-presenting cells (especially dendritic cells) for induction of cytotoxic T lymphocyte-mediated response against tumors. The authors developed PLGA nanoparticles, coated with erythrocyte membrane entraps melanoma-associated antigenic peptide and targets dendritic cells because of their ability to specifically target tumor antigens that promote secretion of pro-inflammatory cytokines such as TNF- $\alpha$ and IFN- $\gamma$ and causes enhanced CD8+ T-cell response. This novel antigenic peptide delivery system retarded tumor growth and suppressed tumor metastasis in a prophylactic, therapeutic, and metastatic melanoma model. This illustrates the possibility of using erythrocytes derived membranes in the development of biomimetic nanoparticles to demonstrate tumor-specific immune response [156]. Hybrid nanoparticle infusing two types of bioinspired membranes: erythrocytes and platelets for prolonging circulation time and the other for expression of adhesion proteins, respectively which increases site-specific targetability and prevents unwanted macrophage uptake This study demonstrates the ability of fusing two different types of cell membrane into one nanoparticle 
construct that will possess individual properties of each type of membrane and the endless number of possibilities that could be further explored [157].

\section{Neutrophil membrane-formed nanoparticles}

Neutrophils are the most commonly found leukocytes in humans. They are the first immune cells that migrate to any infected site caused by bacteria and virus, to prevent the pathogens from spreading, by the release of cytokines/chemokines and ROS production. This response is called acute inflammation and involves neutrophil infiltration, which is regulated by intercellular interaction due to the presence of adhesion molecules on the neutrophils and vascular endothelium [158]. For example, researchers have demonstrated how activated neutrophils which express integrin $\beta_{2}$ binds to the inflamed vasculature that overexpress ICAM-1 [159]. A similar approach of conjugating nanoparticles to anti-ICAM-1 has also been explored by various researchers, however, the conjugation process did not show significant targeting improvement due to the complex tumor microenvironment [160]. Also, the cost ineffectiveness makes this strategy less useful and makes avenue for exploring the other potential options for novel targeting approach, such as cell membrane-based or whole-cell nanoparticles. Gao et. al.[161] shows how human leukemia HL-60 cells was utilized for generating nanovesicles by nitrogen cavitation method, which highly express integrin $\beta_{2}$ and binds to the inflamed vascular endothelium due to the overexpression of ICAM-1 on them. The authors loaded the nanovesicles with an anti-inflammatory drug (TPCA-1) to show the ability of these nanovesicles to reduce the expression of cytokines TNF- $\alpha$ and IL-6, demonstrating their ability to bind to the inflamed vasculature and producing an anti-inflammatory effect. Red blood cells were used as control to generate nanovesicles that lacked the expression of integrin $\beta_{2}$ and does not bind to the endothelium, elucidating the utilization of neutrophils to deliver therapeutics specifically at inflamed vasculature. Another similar study was demonstrated by Kang et. al. [71] utilizing polymeric nanoparticles coated with the neutrophil membrane to target tumor vasculature and metastatic tumor cells in 4T1 BC with lung metastasis model. The authors demonstrate PLGA nanoparticles when coated with neutrophil membrane and loaded with carfilzomib, a proteasome inhibitor targets the circulating tumor cells or the metastatic niche and the inflamed endothelium. Thus, demonstrating the potential of polymeric nanoparticle coated with neutrophil membrane can be used to inhibit early metastasis and preformed metastasis.

\section{Monocyte-derived nanoparticles}

Monocytes are circulating white blood cells and play a crucial role in the inflammatory response and represent around $10 \%$ of leukocytes. They differentiate into macrophages or dendritic cells, with the latter mainly occurring during the active infection [162]. They also have similar roles as neutrophils and platelets in maintain homeostasis and inflammatory response. Monocytes have 1-3 days half-life and could be exploited due to their intrinsic targeting ability especially at the inflamed vasculature or injury site [163]. Researchers have explored monocytes derived nanovesicles and have shown that these serve as better drug delivery tools over drugloaded exosomes for various disease conditions. Jang et.al. loaded doxorubicin in exosome derived nanovesicles and show similar inhibition in tumor growth as a 20 -fold higher dose of the same drug without causing any systemic adverse effects. The exosomes nanovesicles possess counter receptors such as LFA-1 that has specificity for cell adhesion molecules such as ICAM- 
1, VCAM-1 and E-selectin that is overexpressed on the inflamed endothelium cells, enabling the monocytes to target the circulating cells causing the maximal release of the therapeutic cargo at the targeted site [164]. On a similar approach, PLGA nanoparticles loaded with doxorubicin and coated with monocytes that express $\alpha 4 \beta 1$ integrin and binds to the cell adhesion molecules such as VCAM-1 that is overexpressed on the metastatic cancer cells have also been explored [165]. Further application of monocytes derived vesicles for the theranostic purpose has also been explored and has shown to cause significantly higher brain uptake of 5 hours over non coated nanoparticles [166]. This type of construct has also been explored for delivery of siRNA and RNAi, due to the failed attempts to deliver naked RNA which gets degraded and cannot pass through the membranes to make it to the targeted site. Thus, this construct has improved delivery of RNA molecules which further can go through the process of RNA interference and suppress overexpressed oncogenes especially in cancer $[167,168]$. The ability of circulating monocytes to target tumor cells due to the expression of protein molecules on the surface of these monocytes, make them specific to the cells that prevent cancer progression, metastasis, angiogenesis, invasion, migration, and resistance to chemotherapy [169].

\section{Platelet membrane-coated nanoparticles}

Platelets are small, non-nucleated cytoplasmic body that is present actively circulating in the blood. They express a wide variety of immune cell receptors and adhesion molecules on their surface, responsible for mediating immune response. The platelets have the unique ability to be recruited instantaneously at the site of injury or after an infection which allows them to bind to the antigens and release blood clotting factors that would heal the wound. The presence of these various protein antigens on their surface allows immunomodulatory and cell adhesion capability. Researchers have utilized platelet membrane coated PLGA nanoparticles in two disease models of coronary restenosis and systemic bacterial infection to deliver docetaxel and vancomycin, respectively. Enhanced therapeutic efficacy was achieved via these novel biomembranes inspired polymeric nanoparticles contrast to uncoated nanoparticles. Also, the authors demonstrate the coating shields the polymeric nanoparticles from unwantedly up taken by macrophages, thus enhancing nanoparticle deposition at the target site [170]. Doxorubicin loaded in platelet coated liposomes with two peptides: GPIIb-IIIa-like integrins and P-selectins expressed on their surface. This construct shows enhanced targetability to bind and destroy specifically metastatic BC cells over nonmetastatic BC cells in in vitro and in vivo models [171]. Platelet coated PLGA nanoparticles that have overexpression of P-selectin was also used to deliver tumor-specific apoptosis-inducing ligand cytokine (TRAIL) and doxorubicin to tumor cells due to specificity for CD44 receptors, expressed on the surface of tumor cells. Due to the enhanced targetability between the platelet membrane and cancer cells, the therapeutic efficacy of TRAIL due to activated extrinsic apoptosis is enhanced resulting in increased apoptosis. This way the authors synergistically deliver active therapeutics to the tumor cells by targeting via both intrinsic and extrinsic pathways, eliminating metastatic cells too [172]. Similar approaches were made by synthesizing silica nanoparticles that were further functionalized with activated platelet membranes and decorated with tumor-specific peptides such as TRAIL. This construct was able to specifically target circulating tumor cells and prevents unwanted phagocytosis, due to the expression of CD47 on the surface of the activated platelets that extends the half-life of the nanoparticles. This targeting strategy binds to circulating metastatic cells and shows significant decrease of lung metastases in metastatic orthotopic BC mouse model [173]. 
Platelets coated magnetic nanoparticles were applied for theranostic applications for both cancer therapy and cancer diagnosis. The authors, fabricated $\mathrm{Fe}_{3} \mathrm{O}_{4}$ nanoparticles coated with platelet membranes that express protein moieties which allows longer circulation and prevents immunogenicity. The application of magnetic nanoparticles allows optical absorption that extends tumor magnetic resonance imaging (MRI) ability and photothermal therapy (PTT). Additionally, PTT allows enhanced tumor targetability along with intrinsic targeting ability from the membrane proteins of the platelets also reducing macrophage uptake. Thus this strategy provides the application of bioinspired nanoparticle for personalized medicine in various disease state [174].

\section{Paclitaxel}

\section{History}

Paclitaxel (PTX) is one of the most common and potent chemotherapeutic agents for the treatment of several types of cancer including BC. It belongs to the taxane family and was first discovered in 1960's from the bark of Pacific yew (Taxus brevifolia) in a plant screening program initiated by the National Cancer Institute (NCI) of the United States. Later in 1971, scientists named it Taxol and later the chemical structure was explained [175]. In 1979, the mechanism of action of PTX was discovered by Susan Horwitz as a microtubule stabilizing agent [176]. After showing somewhat promising results in in vivo mouse tumor models in 1978, PTX entered clinical trials for the first time in 1984 [177]. PTX was commercialized by BristolMyers Squibb and attained the trademark name "Taxol ${ }^{\circledR}$ " and was assigned the generic name "PTX" Taxol ${ }^{\circledR}$, so far in history is the most profitable chemotherapeutic drug and the only drug to be selected from plant screening program $[177,178]$. Initially, PTX was extracted from Taxus bark, however, to yield 2 grams of the potent compound 4 trees had to be sacrificed, leading to scarcity of the tree. After tremendous efforts from the medicinal chemist, this complex compound could be synthesized in approximately 40 step reactions by the acylation of 10 deacetylbaccatin III [179]. Taxol ${ }^{\circledR}$ was approved by FDA for BC and non-small cell lung cancer (NSCLC) in 1994 and 1999 respectively [180]. Nowadays, PTX is approved by FDA for Kaposi's Sarcoma (second line), BC (metastatic or non-metastatic), advanced ovarian cancer (first line), microcytic lung malignant neoplasm (metastatic or non-metastatic). Also used in the adjuvant or neoadjuvant setting for esophageal cancer, bladder cancer, prostate cancer, cervical cancer, gastric cancer, head and neck cancer, endometrial malignancy, brain oligodendroglioma and testicular cancer [181].

\section{Properties}

PTX is a tricyclic diterpenoid with a taxane ring and a $\mathrm{C}-13$ side chain that imparts the cytotoxic activity of the compound. It is a white crystalline powder that melts at $216-217^{\circ} \mathrm{C}[182]$ with high lipophilicity of $\log$ P 3.5, making it practically insoluble in water with solubility 0.3 $0.5 \mu \mathrm{g} / \mathrm{ml}$ [183] and thus has low dissolution rate and low oral bioavailability less than $8 \%$ [182]. It is a cytochrome P450-dependent hydroxylase with the carbon at the 2 ' position allows the 
addition of functional groups or polymer conjugation [184], thus increasing activity, as in the case of Taxol $^{\circledR}$.

\section{Mechanism of Action}

PTX binds to the N-terminal 31 amino acids of the beta-tubulin subunit of the microtubule apparatus. PTX promotes microtubules polymerization to maintain the equilibrium between tubular dimers and microtubule polymers and thus stabilizing them. PTX prevents cell proliferation by forming an abnormal organization of mitotic spindle, block at the metaphase/anaphase boundary, as well as incomplete formation of metaphase plate of chromosomes. These effects cause morphologic changes and DNA fragmentation because of the programmed cell death or apoptosis [185].

\section{Taxol ${ }^{\circledR}$ : The First Formulation}

PTX was formulated by Bristol-Myers Squibb, owing to the low solubility, it was formulated in a vehicle of polyoxyethylated castor oil (Cremophor® EL or CEL). This was the first available commercial product of PTX, Taxol ${ }^{\circledR}$ and possessed CEL and absolute alcohol in equal ratio. It is a clear colorless to the slightly yellow viscous solution. Clinical dosage suggests each milliliter of the solution contains $6 \mathrm{mg}$ PTX, $527 \mathrm{mg}$ of CEL and 49.7\% (v/v) absolute alcohol [182]. CEL is an inert vehicle commonly employed for formulating poorly water-soluble drugs. However, it does impart some adverse effects, such as hypersensitivity, nephrotoxicity, neurotoxicity respiratory difficulties, and vasodilatation occurs. Some of the severe effects include anaphylactoid hypersensitivity reactions, hyperlipidemia, abnormal lipoprotein patterns, aggregation of erythrocytes, peripheral neuropathy, bronchospasms and hypotension [186]. It is administered by slow infusion by diluting with a suitable parenteral liquid such as $5 \%$ dextrose. The half-life ranges between 1.3 and 8.6 hours, $90 \%$ of the drug is bound to plasma proteins and possess a large volume of distribution of about $55 \mathrm{~L} / \mathrm{m}^{2}$ [187]. It is primarily metabolized in the liver by the cytochrome P450 (CYP3A4 and CYP2C8) and less than 10\% of the dose is eliminated through the urine. The pharmacokinetics profile relies on the variable infusion time, however, it is considered to be non-linear because of saturation in the process of distribution and elimination [188]. However, due to the smaller change in dose or infusion, systemic exposure may be very high and may result in adverse toxicities. Additionally, CEL is also reported to alter the pharmacokinetic profile of the drug such as the reduction in plasma clearance. Altogether, Taxol ${ }^{\circledR}$ though possesses superior anticancer efficacy, the toxicity issues diminish the sale. Additionally, severe dose-limiting toxicities include myelosuppression and neutropenia [189].

\section{Nano-Based PTX Formulations for TNBC in Preclinical and Clinical Phase}

Nanoparticles have also been utilized particularly in cancer therapy to facilitate accumulation in the tumors via EPR effect, indicating the potential of nanoparticles to deliver chemotherapeutics at the specific site [190] and improve the tolerability of cytotoxic agents [191]. Since chemotherapy still dominates the treatment regimen for TNBC, nanoparticle 
platforms are a strong base for this kind of therapeutics. There is extensive ongoing research to improve the therapeutic benefits of the nanotechnology-based formulation. With this hope, FDA has approved in recent times nanotechnology formulations for PTX and DOX, that has shown improved efficacy yet only show marginal to no improvement with respect to toxicity profile in comparison to the native drug (PTX) such as Abraxane. Abraxane has shown $\sim 2$-fold improvement in overall response rate in patients [136] in contrast to PTX or Taxol ${ }^{\circ}$. However, many investigators have reported the incidence of neuropathy by Abraxane is significantly higher than the native PTX drug [192]. Similar nanoparticle formulations of PTX are GenexolPM, SB05, Lipusu ${ }^{\circledR}$, PNU166945 and many more, were developed and are approved or in clinical trials but failed to cause any significant improvement compared to the currently available treatment modality due to dose-limiting toxicities [193].

\section{Albumin-based nanoparticles: Abraxane or nab-PTX}

The disadvantages associated with Taxol ${ }^{\circledR}$ caused another nanotechnology based PTX formulations to be marketed by Abraxis/Celgene with the tradename "Abraxane". It is a solventfree colloidal suspension that is lyophilized, including six or seven PTX molecules noncovalently bonded to form aggregates of 4-14 nm which further aggregates to form $130 \mathrm{~nm}$ in diameter. It was initially approved by FDA in 2005 for metastatic BC but has eventually been approved for treating NSCLC, metastatic adenocarcinoma and pancreatic cancer, either as a single agent or used in combination with other first-line treatment [191]. Pharmacokinetic profile of Abraxane suggests that clearance and volume of distribution are significantly higher than the traditional formulation. It is well distributed and binds to the tissue and extravascular proteins [194].

Preclinical studies demonstrate an improvement in efficacy, in contrast to Taxol ${ }^{\circledR}$ and $33 \%$ higher tumor accumulation and less toxicity [138]. Thus, the ease of administration, reduction in some of the adverse effects such as hypersensitivity reactions, better overall response, and survival. Although, Abraxane shows a reduced risk of neutropenia yet increased incidence of neurotoxicity is overserved in combination with Gemcitabine in pancreatic cancer [195]. Additionally, a randomized clinical trial CALGB 40502, suggests that toxicity profile for both the treatment groups receiving either PTX or nab-PTX weekly has no improvement, in fact, more neuropathy and myelosuppression was observed with nab-PTX [196].

Clinical trials on BC patients receiving weekly Abraxane shows it is well-tolerated and has higher antitumoral activity in contrast to PTX. However, grade 4 neutropenia and grade 3 peripheral neuropathy were observed as dose-limiting toxicities [197, 198]. Gradishar et. al. conducted a phase III clinical trial on metastatic BC patients with Abraxane showing a significantly higher response rate of $33 \%$ versus $19 \%$ improvement with Taxol ${ }^{\circledR}$. However, no significant difference in overall survival, which is considered one of the most important parameters to evaluate clinical efficacy [199]. Another phase III trial treated with Abraxane ${ }^{\circledR}$ $\left(125 \mathrm{mg} / \mathrm{m}^{2}\right)$ or Taxol ${ }^{\circledR}\left(80 \mathrm{mg} / \mathrm{m}^{2}\right)$ each given weekly, followed by

epirubicin/cyclophosphamide, shows significant higher pcR especially in the TNBC cohort in comparison to Taxol ${ }^{\circledR}$ (38\% vs $29 \%$ ). However, Abraxane ${ }^{\circledR}$ was associated with significantly greater toxicities such as peripheral sensory neuropathy compared with Taxol® $(10 \%$ vs $3 \%)$ [200]. 
No, statistically improvement in the quality of life was noted, although significantly fewer neutropenia ( $9 \%$ vs $22 \%$ ) and more neuropathy (10\% vs $2 \%$ ) were overserved in between groups treated with Abraxane and Taxol ${ }^{\circledR}$ respectively. The most frequent and common side effects seen after treatment with Abraxane was neurotoxicity and neuropathy. These confusing results made researchers wonder about the actual efficacy of the drug. This is when Rugo et. al. decided to investigate the actual therapeutic influence achieved after Abraxane treatment. A phase III clinical trial was conducted to demonstrate treatment difference with either Abraxane $\left(150 \mathrm{mg} / \mathrm{m}^{2}\right)$ or ixabepilone $\left(16 \mathrm{mg} / \mathrm{m}^{2}\right)$, with a once-per-week treatment with to $\mathrm{Taxol}^{\mathbb{B}}(90$ $\mathrm{mg} / \mathrm{m}^{2}$ ) and in combination with the monoclonal antibody bevacizumab. Among the 799 patients enrolled, Abraxane showed lesser tumor response than $\operatorname{Taxol}^{\circledR}(27 \%$ vs $38 \%)$, overall survival (23.5 months vs 26.5 months) and progression-free survival (9.3 months vs 11 months) respectively. Also, Abraxane shows significantly greater hematological and non-hematological toxicity than Taxol ${ }^{\circledR}$. Thus, the failed results from this study clearly bring into the question the actual superiority of this formulation [196]. Altogether, Abraxane though shows some beneficial higher tumor accumulation and improved disease-free survival, there was any significant improvement observed in overall survival of patients in contrast to the gold-standard formulation. Moreover, the heterogeneity of the disease makes treatment to be difficult, especially when Abraxane fails to show any improvement in peripheral neuropathy, in contrast to $\mathrm{Taxol}^{\circledR}$.

\section{Liposomes}

Lipusu $^{\circledR}$ or PTX liposome for injection. The first liposomal PTX-formulation called Lipusu ${ }^{\circledR}$ is communalized by LuyePharma Group in China consists of lyophilized powder of PTX solubilized in liposomes made of lecithin and cholesterol in a ratio of $87: 13 \mathrm{w} / \mathrm{w} \%$ and is $400 \mathrm{~nm}$ in diameter. It is approved in China as first-line chemotherapy for ovarian cancer, firstline therapy for NSCLC patients not suitable for radiotherapy or surgery, and for BC. Wang et.al. investigates the anaphylactic effect of the formulation in contrast to Taxol ${ }^{\circledR}$ showed responses such as piloerection, anhelation, and syncope, which were not seen in animals treated with Lipusu $^{\circledR}$. Although Lipusu ${ }^{\circledR}$ showed some milder hypersensitivity reactions as well [201]. There are several ongoing clinical trials (NCT02142790 for metastatic BC and NCT02142010 for Lipusu with cisplatin in BC) $[202,203]$ to evaluate the efficacy in comparison to other standards available regimen.

\section{LEP-ETU ${ }^{\circledR}$ or liposome entrapped PTX easy to use. Another liposomal PTX}

formulation developed by NeoPharm cardiolipin, cholesterol, and D- $\alpha$-tocopheryl acid succinate (in the molar ration 5:5:90) and are about $150 \mathrm{~nm}$ in diameter. Phase I clinical trial to study the dose-limiting toxicities at $325 \mathrm{mg} / \mathrm{m}^{2}$ associated with the treatment of LEP-ETU ${ }^{\circledR}$ shows peripheral neuropathy, myelosuppression, and even neurotoxicity [204]. Slingerland et. al. demonstrates that the pharmacokinetic profile (AUC and $\mathrm{C}_{\max }$ ) of $L E P-E T U^{\circledR}$ were similar to Taxol ${ }^{\circledR}[205]$. A phase II clinical trial has conducted to determine the safety and efficacy of LEP$\mathrm{ETU}^{\circledR}$ in thirty-five subjects show sensory polyneuropathy $(3 \%)$ and neutropenia $(6 \%)$. Other than that no significant reactions were observed [206].

EndoTAG-1 ${ }^{\circledR}$ or SB05 or MBT-0206 or LipoPac. EndoTAG- $1^{\circledR}$ is a PTX formulation developed by MediGene and later taken over by SynCore Biotechnology. It is comprised of 
liposomes composed of phospholipid DOTAP and neutral phospholipid DOPC in a 53:47 molar ratio and are about $200 \mathrm{~nm}$ in diameter and positive zeta potential of $25-100 \mathrm{mV}$ in a $0.05 \mathrm{mM}$ $\mathrm{KCl}$ solution at $\mathrm{pH} 7.5$ [207]. The positively charged liposomes promote the PTX uptake into the tumor endothelial cells. As a result of which combining antivascular effects with conventional chemotherapy may result in increased permeability within the tumors [208]. However, preclinical and clinical trial conducted on other types of cancer such as adenocarcinoma, pancreatic cancer is shown to cause neutropenia and thrombocytopenia. Efficacy in TNBC was evaluated by Awada and coworkers [96], showing results from a phase II clinical trial comparing the safety and efficacy of EndoTAG- $1^{\circledR}$ with Taxol ${ }^{\circledR}$ in 140 patients, receiving either weekly EndoTAG- $1^{\circledR}$ with Taxol ${ }^{\circledR}$ or EndoTAG- ${ }^{\circledR}$ (twice a week) or weekly Taxol ${ }^{\circledR}$. However, there was no significant improvement in the overall survival between the treatment groups $(13.0,11.9$ and 13.1 months, respectively). Other adverse effects include pyrexia and chills and about 17.8\% discontinued the therapy due to unbearable adverse events. Ongoing clinical trials evaluating EndoTAG- $1{ }^{\circledR}$ for treatment of TNBC (NCT01537536 and NCT00448305) [209, 210].

\section{Polymeric micelles}

Genexol-PM $^{\circledR}$ or Cynviloq ${ }^{\text {TM}}$. Genexol-PM ${ }^{\circledR}$ developed by Samyang Biopharmaceuticals Corporation is the biggest competitor of Abraxane ${ }^{\circledR}$ approved in South Korea for the treatment of several types of cancer, such as breast, ovarian cancer, and NSCLC. In 2013, Sorrento Therapeutics Inc has approved distribution rights in the U.S. and some other countries as well. It is made up of monomethoxy- poly(ethylene glycol)-block-poly (D, L-lactide) diblock copolymer that entraps PTX in polymeric micelles, exhibiting a size range from 20-50 nm with a drug loading capacity of $16.7 \% \mathrm{w} / \mathrm{w}$. The absence of albumin reduces the risk of microbial contamination in comparison to Abraxane. A phase III clinical trial (NCT00876486) of the formulation in contrast to the Abraxane in metastatic BC, shows enhanced efficacy and reduced toxicity issues [211]. Other clinical trials demonstrate that the MTD of this formulation was lowered to $180 \mathrm{mg} / \mathrm{m}^{2}$ than that of Abraxane ${ }^{\circledR}$, although both the regimens showed a similar incidence of neutropenia and less severe non-hemotoxicity [212, 213]. Although, higher-dose regimen of $390 \mathrm{mg} / \mathrm{m}^{2}$ has a higher incidence of neutropenia and neuropathy [214]. In phase II clinical trial by Lee et. al. at a dosing regimen of $300 \mathrm{mg} / \mathrm{m}^{2}$ in metastatic BC patients, $58.5 \%$ of them demonstrate response rates. However, the response rate of Taxol ${ }^{\circledR}$ was similar but conducted in a much larger group. Also, greater incidence of neutropenia (68.3\%), neuropathy $(51.2 \%)$ and hypersensitive reaction $(19.5 \%)$ was observed [215]. Overall, Genexol-PM ${ }^{\circledR}$ showed a similar response to Taxol ${ }^{\circledR}$, which initiated the fast track approval through 505(b)(2) for new drug application (NDA) regulatory pathway for Cynviloq ${ }^{\circledR}$ in the U.S [216].

Paclical $^{\circledR}$. Paclical ${ }^{\circledR}$ developed by Oasmia Pharmaceuticals, which is composed of XR17, a vitamin A derivative which is made of N-(all-trans retinoyl)-L-cysteic acid methyl ester sodium salt and N-(13-cis retinoyl)-L-cysteic acid methyl ester sodium salt. It forms micelles of 20-60 nm which encapsulates PTX and is available as a lyophilized powder, administered as infusion [217]. Pharmacokinetic profile of Paclical ${ }^{\circledR}$ and Abraxane ${ }^{\circledR}$ are similar, suggesting the opportunity for approval of Paclical in the treatment of metastatic BC [218].

Nanoxel ${ }^{\circledR}$ or DO/NDR/02. Nanoxel ${ }^{\circledR}$ from Dabur Pharma Limited is available for the treatment of metastatic BC, ovarian cancer, NSCLC and AIDS-related Kaposi's sarcoma in 
India. It is a micellar solution of size range $80-100 \mathrm{~nm}$, is composed of a $\mathrm{pH}$-sensitive copolymer of $\mathrm{N}$-isopropyl acrylamide and vinylpyrrolidone monomers which is stable at physiological $\mathrm{pH}$ and drug release in an acidic environment as tumor microenvironment [219]. Ranade et. al demonstrates in phase I clinical trial the MTD to be $375 \mathrm{mg} / \mathrm{m}^{2}$ with a linear pharmacokinetic behavior with grade 3 diarrhea and grade 4 neutropenia adverse effects [220]. However, the same group conducted a phase II trial in anthracycline failed advanced metastatic BC patients and show though the formulation in comparison to the generic formulation Taxol ${ }^{\circledR}$ has improved overall response rate, being $40 \%$ and $32.3 \%$, the higher incidence of neutropenia of $56.3 \%$ versus $50 \%$, higher incidence of neuropathy of $12.5 \%$ versus 6.3 , respectively [219].

Triolimus $^{\circledR}$. Triolimus ${ }^{\circledR}$ from Co-D Therapeutics is a polymeric micellar formulation composed of poly(ethyleneglycol)-block-poly(lactic-acid; PEG-PLA) and encapsulates three drugs, PTX, rapamycin which is mTOR inhibitor and Hsp90 inhibitor tanespimycin (17-AAG) ranging about 30-40 $\mathrm{nm}$ [221]. After showing promising preclinical results in xenograft models by the synergistic activity of the three actives, this formulation is in the process of phase I clinical trial for breast, NSCLC, and angiosarcoma [222].

NK105. NK105 is a micellar formulation composed of polyethylene glycol and modified polyaspartate by esterification with 4-phenyl-1-butanol of about $85 \mathrm{~nm}$ in diameter [223]. The core encapsulates $23 \%(\mathrm{w} / \mathrm{w})$ of PTX and demonstrates good drug retention after intravenous. administration. This formulation has MTD of about $180 \mathrm{mg} / \mathrm{m}^{2}$ in phase I clinical trial with grade 3 neutropenia as the adverse dose-limiting toxicity. Pharmacokinetic profile of this formulation in comparison to the marketed generic formulation Taxol ${ }^{\circledR}$ has 15 folds higher AUC at the recommended dose of $150 \mathrm{mg} / \mathrm{m}^{2}$. However, in phase III clinical trial against metastatic BC patients showed failed to meet the primary endpoint of the study [224].

\section{Polymeric-lipidic nanoparticle: PICN or PTX injection concentrate for nanodispersion}

PICN is a nanoparticle-based formulation composed of polymer and lipid, polyvinylpyrrolidone, cholesteryl sulfate and caprylic acid-forming nanoparticle of 100-110 nm, developed by Sun Pharma and approved in India for the treatment of metastatic BC. Jain et. al evaluated the efficacy of this formulation in comparison to Abraxane in phase II/III clinical study and demonstrate similar efficacy and tolerability profile at MTD of $260 \mathrm{mg} / \mathrm{m}^{2}$. Although the incidence of most common side effects such as neutropenia, peripheral neuropathy, and leukopenia was the least at MTD $260 \mathrm{mg} / \mathrm{m}^{2}$ versus PICN $295 \mathrm{mg} / \mathrm{m}^{2}$ and Abraxane $260 \mathrm{mg} / \mathrm{m}^{2}$, yet there was no statistically significant difference between them [225]. In U.S. PICN is in phase III clinical trial for the treatment of biliary tract carcinoma (NCT02597465) [226] and just finished phase I trial when administered alone or in combination with carboplatin (NCT01304303), the results are yet to be disclosed [227]. 


\section{Polymeric conjugates}

HPMA copolymer-PTX (PNU166945). PNU166945 is the first polymer-drug conjugate of PTX, developed by Pharmacia Corporation, conjugating PTX to HPMA by a tetrapeptidil linker of glycylphenylalanylleucylglycine. Although the formulation has good aqueous solubility and about $5 \% \mathrm{w} / \mathrm{w}$ drug loading, it failed to have any difference in pharmacokinetic behavior, illustrating that the conjugation did not have any significant impact on the drug behavior, though a partial response was observed in one of the twelve patients with advanced BC. However, clinical studies in phase I was discontinued earlier due to severe neurotoxicity observed [228].

Opaxio $^{\text {TM }}$ or PTX Poliglumex, CT-2103 or Xyotax $^{\circledR}$. Opaxio ${ }^{\mathrm{TM}}$ is a polymer drug conjugate of PTX conjugated to the $\alpha$-poly (L-glutamic acid) at the 2 '-hydroxyl position of the drug by an ester linkage. It is available as a lyophilized powder and has shown beneficial results in preclinical studies. In ovarian tumors, uptake of Opaxio ${ }^{\mathrm{TM}}$ was about 5-fold higher than Taxol ${ }^{\circledR}$ with prolonged circulation time [229]. After promising preclinical results, pharmacokinetic profiling of this compound in comparison to the original drug was conducted by Boddy et. al and shows prolonged half-life and low renal clearance, however, the achieved $\mathrm{C}_{\max }$ was 3 times lower than Taxol ${ }^{\circledR}$. Also, similar dose-limiting toxicity such as neuropathy and neutropenia similar to taxanes was observed [230]. CT-2103 completed phase II trial for metastatic BC [231] and is also being evaluated in combination with carboplatin versus PTX and carboplatin in NSCLC patients [232].

\section{Emulsion}

DHP107. DHP107 is an emulsion composed of PTX, monoolein, tricaprylin, and Tween 80, in a ratio of 1: 55: 27.5: $16.5 \mathrm{wt} \%$ and developed by Daehwa Pharmaceutical in South Korea. Phase I clinical trial demonstrates that is well tolerated in patients up to $600 \mathrm{mg} / \mathrm{m}^{2}$ with no adverse effects of neutropenia. It is approved in South Korea for the treatment of gastric cancer and is the first product that has shown similar efficacy profile via an oral route in comparison to intravenous administration of Taxol ${ }^{\circledR}$. In the U.S. it is undergoing phase II clinical trial as an oral administration in comparison to the parent drug in metastatic BC patients (NCT03326102) [233].

Tocosol $^{\text {TM }}$. Tocosol ${ }^{\mathrm{TM}}$ is an emulsion of vitamin E, TPGS, and poloxamer-407 with PTX formulated in the oil in water emulsion, bearing a particle size of $100 \mathrm{~nm}$ and high drug loading capacity, this formulation is shown beneficial results in preclinical studies [234]. However, in phase III clinical trial it was retracted, due to a similar response rate as Taxol ${ }^{\circledR}$ in metastatic BC patient [235].

\section{Peptide-conjugates: ANG 1005}

ANG 1005 is a drug peptide conjugate of angiopep-2 and PTX that binds to the lowdensity lipoprotein receptor-related protein (LRP)-1 which facilities movement across the bloodbrain barrier (BBB), as LRP-1 is highly overexpressed in BBB. In preclinical model ANG 1005 has shown therapeutic efficacy which has progressed its application in a clinical trial. In phase II trial among metastatic $\mathrm{BC}$ patients promising results have been achieved with the application of 
ANG1005 to treat peripheral metastatic BC and the brain metastasis [236]. It is now in phase III clinical trial (NCT03613181) [237].

\section{Conclusion}

To date, TNBC remains a disease with poor prognosis and poorer patient outcomes because of the disparity, in terms of molecular and genomic profiles among TNBC classification. Although major advancement has been made with targeted therapy for other types of BC, such as for HER2 positive where trastuzumab has proven to be a blessing, yet in the case of TNBC chemotherapy remains the backbone treatment regimen. A ray of hope has recently emerged among TNBC patients when FDA approved the first-ever targeted therapy: PARP inhibitors, olaparib and talazoparib in 2018. Although it is restricted among patients with BRCA1/2 mutation, accounting for only $10-15 \%$ among the TNBC population, extensive research is ongoing leading to positive preclinical and clinical outcomes with combination therapy or targeted therapy or immunotherapy or ADC. Still, a lot of improvement could be achieved with other novel and/or nano formulation-based therapies, such as Abraxane, that could lead to greater hope for enhancing treatment options and health outcomes for TNBC patients. With this aim to develop a therapy that could be beneficial to the TNBC population, we selected the most commonly used chemotherapeutic agent PTX and developed a nanoparticle-based formulation. We further developed and studied this PTX nanoformulation and explored it for generating biomimetic cell membrane-based nanoparticles, which due to its innate sleath properties improve circulation time, improve the chance of the particulate drug getting into the tumor via the EPR. These membranes are also immunocompatible, thus preventing non-specific adverse effects.

\section{Specific Aims}

A fundamental problem in the development of targeted therapy for TNBC is the lack of expression of ER, PR, and HER2. Due to the lack of a prognostic marker, their aggressive nature and heterogenicity, the mainstay treatment is systemic administration of cytotoxic drugs. These potent yet cytotoxic agents pose adverse effects on non-targeted healthy tissues with low penetration of drugs at the primary tumor or the metastatic region. One such commonly used agent is PTX which is used as a gold standard treatment to tackle TNBC.

We aim to develop a novel therapy using PTX that can, not only have therapeutic benefits but also possesses specificity and targeting ability to the cancer cells. The central hypothesis of this proposal is to develop and characterize an effective drug delivery system that can efficiently target the primary tumor site, thus, maximize the therapeutic window by allowing enhanced targeting specificity and minimize off-targeting problems, which usually causes low therapeutic response with conventional drug delivery. For this, we have designed three sub- aims that would lead us to achieve the final aim of this research. 


\section{Aim 1: To Improve Paclitaxel-Mediated Delivery, by Developing and Characterizing Polymer-Based Self-Assembled Paclitaxel Nanoparticles for BC}

The hypothesis of this aim is the utilization of the self-assembly process, which has shown to cause synergistic interactions between self-organizing particles and leading to hierarchically ordered structures. With this motive, we aimed to develop an effective delivery system for BC cells by self-assembly formation, which will minimize any other formulator/external agents. We screened a wide array of marketed pharmaceutical polymers to determine the best nanoparticle-based self-assembly of gold standard chemotherapeutic agent, PTX. This aim will help us select the nanoparticle-based therapeutics for improving the PTX mediated delivery.

\section{Aim 2: To Develop and Characterize Nanoparticle-Based Paclitaxel Formulation to Enhance Therapeutic Benefits Using P-gp Efflux Inhibitor, such as Tannic Acid in TNBC}

The hypothesis for this aim is based on our initial finding that suggests a self-assembly process for the generation of PTX nanoparticle-based formulation as an effective drug delivery system, that not only enhances the therapeutic efficacies of PTX, but can improve drug efflux limitations, causing subtherapeutic systemic concentration. Therefore, in this aim, we screened common pharmaceutical excipients for generating the effective nanoparticle-based system for TNBC that has chemosensitization motifs and has the potential to enhance the therapeutic effect of PTX. This aim will generate and characterizing nanoparticle-based formulation of tannic acid, which is a known inhibitor of P-gp efflux pumps.

\section{Aim 3: To Enhance Targetability of Paclitaxel in TNBC Tumors by Developing and Characterizing Biomimetic Nanoparticles Using Endogenous Cell Membrane, such as Human Neutrophils}

The hypothesis for this aim is to develop and characterize a specific delivery system that targets TNBC tumors and reduce off-target toxicities on healthy cells. For this aim, we have screened endogenous cells and develop, characterize the developed biomimetic nanoparticles from these endogenous cell membranes to achieve targetability to the TNBC tumors. This strategy potentiates biocompatibility and prevents clearance by the innate immune system which recognizes conventional nanoparticles as immunogenic. The presence of abundant protein moieties present on the surface of the extracted cell membranes allows cell-specific adherence and targetability to primary TNBC tumors, allowing prolong circulation, retention at the tumor site and reduce adverse effects. 


\section{CHAPTER 2. DEVELOPMENT OF POLYVINYL/PYRROLIDONE-PTX SELF- ASSEMBLIES FOR BC ${ }^{1}$}

\section{Introduction}

As discussed earlier BC remains the most commonly diagnosed cancer among women in the United States [238]. [238]. PTX is a natural compound, (derived from the bark of Taxus t56) antimicrotubule and well-established chemotherapeutic agent, which exhibits a broad spectrum of anticancer activity against BC, prostate cancer, leukemia, non-small cell lung cancer, and ovarian cancer $[239,240]$. Due to its hydrophobicity, it is often formulated with cremophor EL in ethanol solution, i.e. Taxol ${ }^{\circledR}$. This limits its clinical use due to its adverse side effects, such as increased risk for fatal hypersensitivity reactions [241], non-linear pharmacokinetics [242], prolonged peripheral neuropathy [186], and myelosuppression [243], less bioavailability at the tumor site and therefore reduced efficacy for its action against tumor cells [186, 244, 245]. Other common side effects of PTX treatment include vomiting, nausea, loss of appetite, and joint pain.

To circumvent these side effects, various alternative nanoparticle-based PTX formulations were developed. Among them, one of the most widely used formulations is Abraxane ${ }^{\circledR}$, which is a cremophor-free and albumin-bound PTX nanoparticle formulation (diameter $\sim 120 \mathrm{~nm}$ ), developed as an alternative formulation to PTX $\left(\right.$ Taxol $^{\circledR}$ ) [239]. This serum albumin-bound PTX nanoformulation is approved by the FDA for the treatment of breast, lung and pancreatic cancer. This formulation facilitates crossing across endothelial layers of cells and achieves 33\% higher PTX concentration at the tumor site [246]. Additionally, Abraxane ${ }^{\circledR}$ was able to increase the maximum tolerated dose (MTD) by almost 70-80\% when compared to PTX alone. Another PTX nanoformulation called Genexol PM ${ }^{\circledR}$ was approved in South Korea as a first-line treatment for metastatic or recurrent BC, non-small cell lung cancer, and also used in combination with carboplatin for ovarian cancer. It is a polymeric micellar formulation of polyethylene glycol (PEG), poly (DL-lactide) and PTX. NK-105 (block copolymer of PEG and polyaspartate modified with 4-phenyl-1-butanol with PTX) is another nanoparticular micellar formulation that has entered phase III clinical trial for metastatic and recurrent BC. NK-105 prolonged circulation, the enhanced area under the curve (AUC) by 20 times in contrast to PTX and also has higher antitumor activity. [223]. Other clinically used (or under clinical development) PTX formulations/nanoformulations include Paclical ${ }^{\circledR}$ (PTX combined with Oasmia's excipient technology XR17) [247], Lipusu ${ }^{\circledR}$ (PTX liposome) [248], PTX injection concentrate for nanodispersion (PICN) [225], SB05 (positively charged liposome embedded with PTX) [249], LEP-ETU (liposome-entrapped PTX) [250], and Triolimus (micelle containing PTX, rapamycin and 17-AAG) [251]. Self-assembly and solid dispersion techniques were a common approach to generate these clinically relevant PTX nanoformulations.

Therefore, the goal of this study was to develop a PTX nanoformulation using a polymerbased self-assembly technique, using a polymer excipient already in use in the pharmaceutical

\footnotetext{
${ }^{1}$ Modified from final submission with open access permission. Chowdhury, P., Nagesh, P. K., Khan, S., Hafeez, B. B., Chauhan, S. C., Jaggi, M., \& Yallapu, M. M. (2018). Development of polyvinylpyrrolidone/paclitaxel selfassemblies for breast cancer. Acta pharmaceutica sinica B, 8(4), 602-614 https://doi.org/10.1016/j.apsb.2017.10.004
} 
industry. The self-assembly process is a well-established, simple, and rapid fabrication method to generate nanosized architecture materials for drug delivery applications [252-254]. We have selected 8 biocompatible polymers. Table 2-1 provides structures of polymers used in this study. Among which Poloxamer 188 [a nonionic copolymer containing hydrophobic poly(propylene oxide) (PPO) and two hydrophilic poly(ethylene oxide) (PEO) units] is a well-known option for generating micelles by the self-assembling technique where the PEO units align at the outer lining and PPO falls in the inner core leading to the formation of micelle above the critical micellar concentration. We expect polymeric micelles will be formed using Poloxamer 188 [252]. Whereas, the rest seven polymers are commonly used as drug delivery carriers for generating solid dispersions or polymeric nanoparticles [253-255]. The motive of this study is to minimize any other external agents being added for the formulation development. Self-assembly is a process where the various components are held together by inter-particulate assembly [256]. Reports are stating that synergistic interactions between self-organizing particles and a selfassembling matrix material can lead to hierarchically ordered structures [257]. Accordingly, we developed nanostructured formulations of hierarchical order using PTX and various polymers without the use of any external reagents or binders. Such self-assembly formulations may have the potential to circumvent the shortcomings of PTX and provide advantages such as ease of preparation; smaller particle size; an efficient binding ability to PTX; and enhanced particle uptake in cancer cells. In this investigation, we report an optimized PVP/PTX-SA formulation which has demonstrated enhanced cellular uptake and exhibited superior in vitro cytotoxicity in $\mathrm{BC}$ cells, compared to PTX.

\section{Materials and Methods}

\section{Chemicals and Solvent}

All laboratory reagents, solvents, and chemicals were purchased from Sigma-Aldrich Co. (St. Louis, MO, USA) or Fisher Scientific (Pittsburgh, PA, USA) unless otherwise mentioned. Cell culture plastics were purchased from Sarstedt, Inc. (Newton, NC, USA). All chemicals were used as received without any further purification.

\section{Cell Culture, Growth Conditions and Treatment}

BC cell lines (MCF7 and MDA-MB-231) were obtained through American Type Culture Collection (Manassas, VA, USA) and stored at low passage frozen aliquots upon cell culture expansion. These cell lines were thawed and cultured under sterile conditions for all experiments ( $<3-4$ months after thawing). Both cell lines were cultured in Dulbecco's Modified Eagle Medium-High Glucose (DMEM-Hi) medium containing $4.5 \mathrm{~g} / 1$ of glucose, $10 \mathrm{nM}$ of nonessential amino acids, $100 \mathrm{nM}$ of sodium pyruvate, $1 \mathrm{x}$ antibiotic/antimycotic (Gibco, Thermo Fisher Scientific, Grand Island, NY, USA) and 10\% heat-inactivated FBS (Atlanta Biologicals, 
Table 2-1. Polymers employed for self-assembling PTX to produce polymer/PTX selfassembly nanoparticles

Name of polymer

Polyvinyl alcohol (PVA)

Structure of polymer

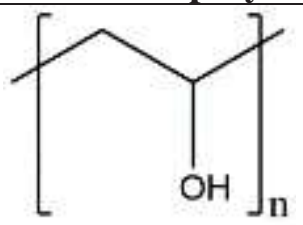

Polyethyleneimine (PEI)

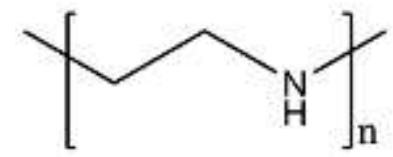

Poly (methyl vinyl ether-altmaleic hydrochloride) (PMEAVH)

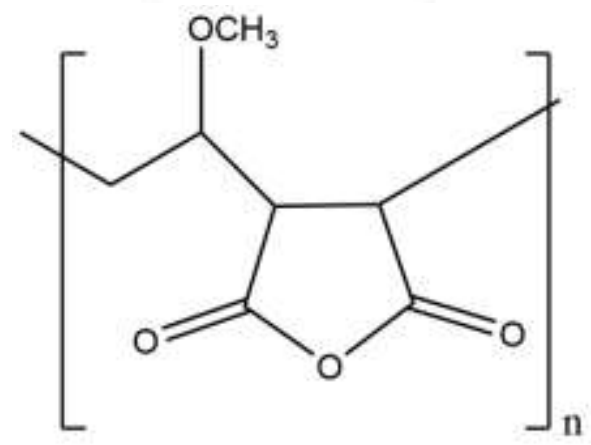

Poly (allylamine hydrochloride) (PA-HCl)

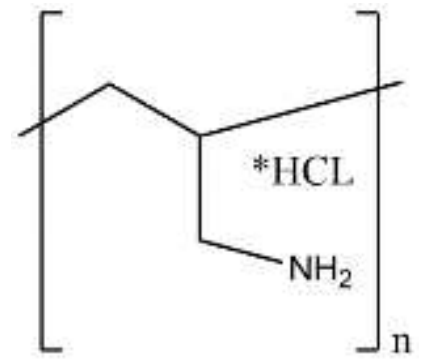

Polyacrylic acid (PAA)

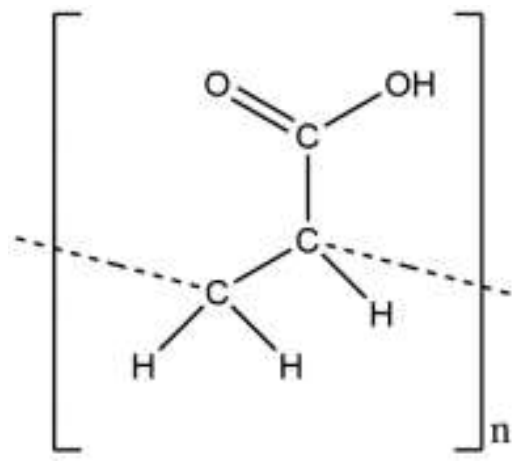


Table 2-1. (Continued)

Name of polymer

Polyvinylpyrrolidone (PVP)

Povacoat F (polyvinyl alcoholacrylic acid-methyl

methacrylate) copolymer

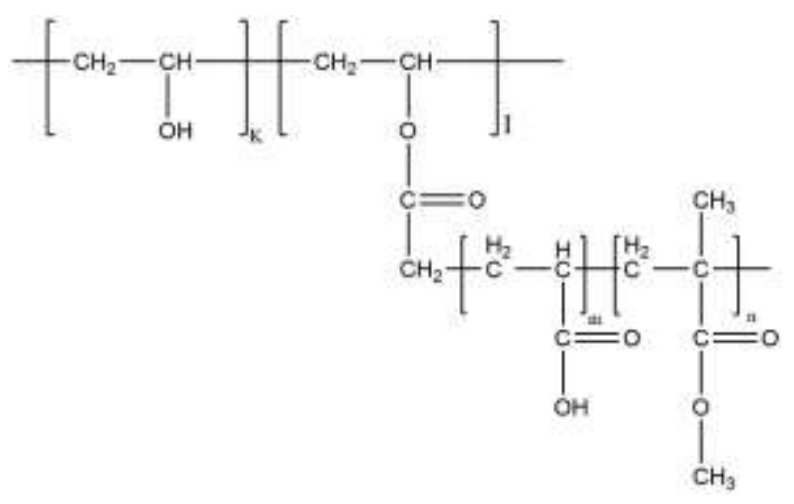

Poloxamer 188 (P188)
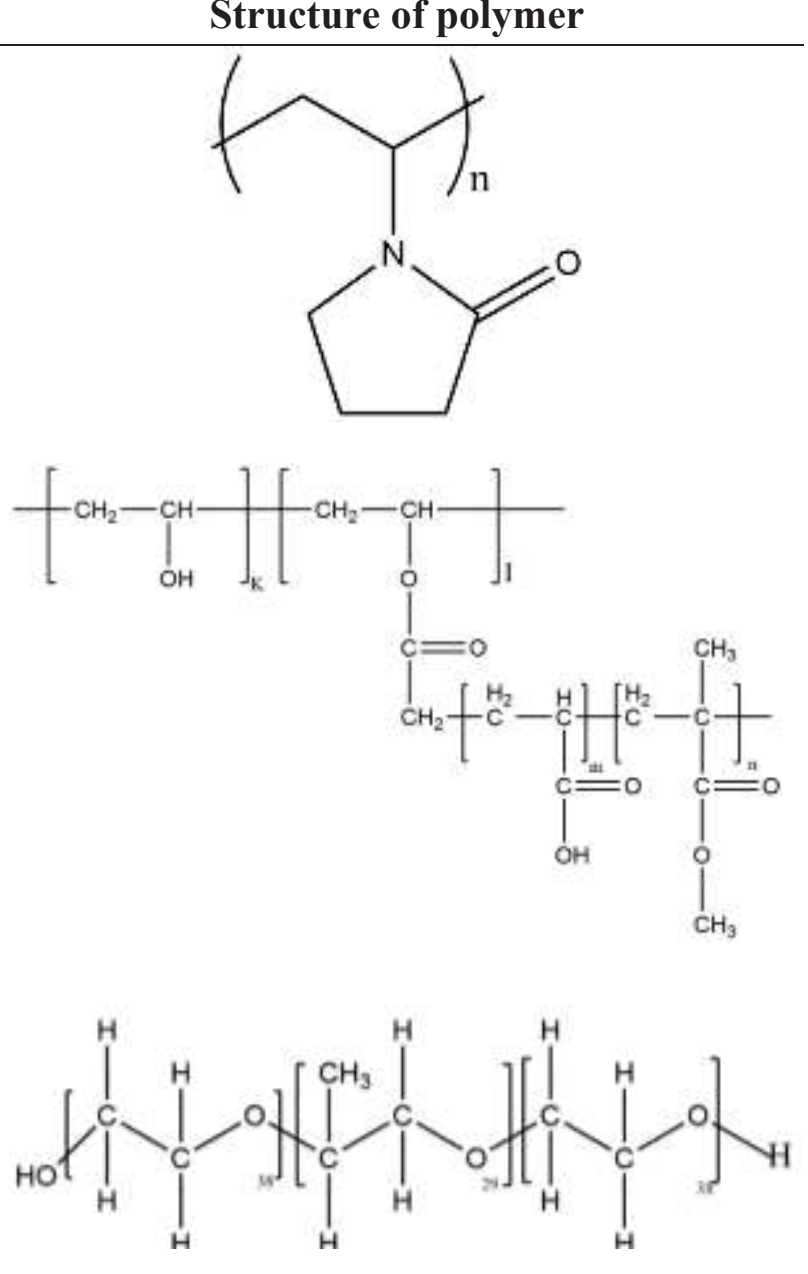
Lawrenceville, GA, USA) at $37^{\circ} \mathrm{C}$ in a humidified atmosphere $\left(5 \% \mathrm{CO}_{2}\right.$ and $95 \%$ air condition) (ThermoScientific, Waltham, USA). In all cell culture experiments, monodispersed cell lines after trypsinization were plated on either 6,12 , or 96 -well plates, and were allowed to adhere overnight to the plate before implementing treatments.

\section{Preparation of Polymer-PTX-SAs (Poly/PTX-SAs)}

Eight polymer-based drug delivery vehicles such as polyvinyl alcohol (PVA) (Mw 31,000-50,000), polyvinylpyrrolidone (PVP) (Mw 40,000), polyethyleneimine (PEI) (Mw 25,000), poly (methyl vinyl ether alt-maleic hydrochloride) (PMEAVH) (Mw 216,000), poly(allylamine hydrochloride) (PA-HCl) $(\mathrm{Mw} 17,500)$, Poloxamer 188 (Kolliphor ${ }^{\mathbb{B}} \mathrm{P} 188$, P188), polyacrylic acid (PAA) (Mw 100,000), and Povacoat (Polyvinyl alcohol-Acrylic acidMethyl methacrylate copolymer: Type $\mathrm{F}, \mathrm{Mw}=40,000$ ), a gift sample received from Daido Chemical Co., Osaka, Japan), were used in the PTX self-assembly formation. The self-assembly formation technique is followed by solvent evaporation. Briefly, $5 \mathrm{mg}$ of polymer was dissolved in $1 \mathrm{ml}$ aqueous medium in $8 \mathrm{ml}$ glass vial under continuous stirring at $400 \mathrm{rpm}$ on a stir plate. To this solution, $100 \mu 1$ of PTX dissolved in acetone $(1 \mathrm{mg} / \mathrm{ml})$ was added slowly (dropwise) and the speed increased to $900 \mathrm{rpm}$. The obtained solution was stirred overnight to evaporate the acetone resulting in the formation of self-assembled nanoparticles comprised of assembled polymer chains and PTX, i.e., referred to as polymer-PTX-self-assemblies (Poly/PTX-SAs). The resultant Poly/PTX-SAs solution was kept refrigerated and used within a week. For all in vitro cell culture experiments, a fresh batch of Poly/PTX-SAs was used to protect PTX activity in the formulations.

\section{Characterization of Poly/PTX-SAs}

\section{Particle size and zeta potential}

The average particle size, particle distribution and zeta potential of the prepared PTX-SA formulations were measured by the dynamic light scattering (DLS) principle using Zetasizer (Nano ZS, Malvern Instruments, Malvern, UK). These measurements were done as previously reported [258], briefly $50 \mu \mathrm{l}$ of freshly prepared Poly/PTX-SA nanoparticle suspension was dispersed in deionized water and was probe sonicated (VirSonic Ultrasonic Cell Disrupter 100, VirTis) for 30 secs. Probe sonication procedure is commonly applied in formulation development for the formation of nanoparticles and homogeneous solution which minimizes the interaction of aggregated nanoparticles and to disperse well but not to break particles into smaller particles. Additionally, the short span of sonication is subjected to ensure that particle suspension is homogeneous, thus warrants there is no agglomeration occurring in particle suspension. Particle size measurements were performed in water for $3 \mathrm{mins}$ at $25^{\circ} \mathrm{C}$. The average diameter and distribution of particle size were determined from three runs. Similarly, zeta potential was measured using nanoparticle suspension that was diluted with $1 \mathrm{X}$ phosphate-buffered saline (PBS). The zeta potential of nanoparticle formulations was also based on the average of 3 readings (each reading $=30$ runs). 


\section{Transmission electron microscopy}

The size and surface morphology of PVP/PTX-SAs were investigated by JEOL 200EX transmission electron microscopy (TEM) (JEOL Ltd, Tokyo, Japan) operating at $80 \mathrm{kV}$. For this, $100 \mu 1$ of PVP/PTX-SA suspension was dispersed in $1 \mathrm{ml}$ of water were probe sonicated for 30 secs. On a 150-mesh standard TEM grid (Electron Microscopy Sciences, PA, USA), a $20 \mu 1$ aliquot of the PVP/PTX-SAs was carefully placed on the shiny side of the grid. It was stained using $2 \% \mathrm{w} / \mathrm{v}$ uranyl acetate solution. The excess amount of formulation was removed using filter paper and the grid was allowed to air dry followed by imaging using an AMT camera at a direct magnification of 100,000X under the TEM system.

\section{Spectral analysis}

Fourier transform infrared (FTIR) spectra were employed to determine the formation of PVP/PTX-SAs. FTIR spectral data were acquired on the Universal ATR sampling Accessory plate using a Spectrum 100 FTIR spectrophotometer (Perkin Elmer, Waltham, MA). Samples of PVP/PTX-SAs was lyophilized to obtain dry solid particles using a Labconco Freeze Dry System $\left(-48^{\circ} \mathrm{C} 133 \times 10^{-3} \mathrm{~m}\right.$ Bar; Labconco, Kansas City, MO, USA). The samples (PTX, PVP and PVP/PTX-SA) were placed on the tip of the ATR objective and spectra obtained between 4000 and $650 \mathrm{~cm}^{-1}$.

\section{Fluorescence Binding}

Self-assembly formation of polymer and PTX was determined on a SpectraMax Plus plate reader (Molecular Devices, Sunnyvale, CA, USA). For this study, PTX conjugated dye (PTX, Oregon Green 488 conjugate with a Flutax-2, Molecular Probes Inc., Life Technologies, OR, USA) was used, from which the fluorescence quenching of Oregon green 488 on PTX was achieved by titrating with polymeric/surfactant solutions. Intrinsic fluorescence of $5 \mu \mathrm{g} / \mathrm{ml} \mathrm{PTX}$ solution in 1X PBS was titrated with $0-80 \mu \mathrm{g} / \mathrm{ml}$ of PVP solution. The decay in fluorescence was studied by exciting at $496 \mathrm{~nm}$ and emission between 450-600 $\mathrm{nm}$.

\section{Hemolysis Assay}

A healthy human donor blood with sodium citrate as anticoagulant was purchased from Interstate Blood Bank Inc (Memphis, TN, USA) for the hemolysis test. The hemoglobin releases from red blood cells (RBCs) upon treatment with $10 \mu \mathrm{g}$ PTX/well or $10 \mu \mathrm{g}$ PTX equivalent/well of Poly/PTX-SAs were quantitatively measured as described earlier $[262,263]$. For this assay, $200 \mu \mathrm{l}$ of RBC suspension $(8 \times 105)$ was incubated with $10 \mu \mathrm{g} / \mathrm{well}$ of PTX or Poly/PTX-SAs (of similar concentration as PTX) and placed in an incubator for $60 \mathrm{mins}$ at $37^{\circ} \mathrm{C}$. In this experiment, sodium dodecyl sulfate (SDS, $1 \mathrm{mg} / \mathrm{ml}$ ) served as a positive control (100\% lysis) while 1X PBS was used as a negative control. After the incubation of 1 hour, hemoglobin release into medium (supernatant, $100 \mu \mathrm{l}$ ) was collected, transferred to a 96-well plate, recorded absorbance at $510 \mathrm{~nm}$ using a plate reader (Cytation 3 imaging reader, BioTeK Winooski, VT, USA). To further confirm our results qualitatively, a few drops of the treated RBC solution was 
smeared on a glass slide and images were captured using EVOS ${ }$ FL Imaging System (AMF4300, Life Technologies, Carlsbad, CA, USA). The percentage of hemolysis was calculated using the following Equation 2-1.

$$
\% \text { Hemolysis }=\left[\frac{\text { Absorbance of samples-Absorbance of negative control }}{\text { Absorbance of positive control }}\right] X 100 \text { (Eq. 2-1) }
$$

\section{Cellular Uptake}

For this study, previously reported methods were employed for qualitative and quantitative uptake of Coumarin 6 (C6) loaded in Poly-SAs in cancer cells [259, 260]. In this experiment, freshly prepared C6 loaded Poly-SAs were generated following the procedure as described in section 2.3. The polymer and dye ratio were kept 50:1 (5 mg of polymer and $100 \mu \mathrm{g}$ of dye). BC cell lines MCF7 and MDA-MB-231 (5000 cells/well with $100 \mu 1$ media/well in 96well culture plates) were seeded at 500,000 cells/well with $2 \mathrm{ml}$ medium in 6 -well plates. Cells were allowed to attach to the plate, media was replaced with $5 \mu \mathrm{g}$ (dye equivalent) C6 loaded Poly-SAs. To ensure the stability of C6 dye, such that it does not leach out of the nanoassemblies, a lower incubation time of 3 hours was selected. Since it is a comparative experiment among 8 different formulations to find out the best one, we have not considered C6 leaching from formulations. After 3-hours treatment, cells were washed twice with 1X PBS to remove the excess C6 loaded Poly-SAs that may be adhered to the surface of the culture plate or cells. Then, DMEM phenol red free medium was added to these treated cells and uptake of C6 loaded Poly-SAs in cells were imaged using EVOS ${ }^{\circledR}$ FL Imaging System (AMF4300, Life Technologies, Carlsbad, CA, USA). Furthermore, the quantitative uptake of C6 loaded Poly-SAs in cells was determined by trypsinizing, centrifuging and then collecting the cells in $2 \mathrm{ml}$ media, which were injected (50 $\mu 1$ cell suspension) into an Accuri C6 Flow Cytometer (Accuri Cytometer, Inc., Ann Arbor, MI, USA). Quantitative uptake of Couamrin 6-SAs measurements were acquired using the fluorescence levels in FL1 channel (488 excitation, Blue laser, $530 \pm 15$ $\mathrm{nm}$, FITC/GFP). Standard deviations were calculated from 3 replicates.

\section{Biological Assay}

\section{Cell viability assay}

A calorimetric CellTiter $96 \AA$ aqueous one solution cell proliferation assay (MTS assay, Promega, Madison, WI) was used to examine the effect of PTX-SAs on cell proliferation of BC cells. The measurement of cell viability is based on the ability of viable cells to cleave the tetrazolium compound [3-(4,5-dimethylthiazol-2-yl)-5-(3-carboxymethoxyphenyl)-2-(4sulfophenyl)-2H-tetrazolium, salt by mitochondrial dehydrogenase. Briefly, MCF7 and MDAMB-231 (5000 cells/well with $100 \mu \mathrm{l}$ media/well in 96-well culture plates) were seeded in regular growth medium and allowed overnight cells to attach to the wells. These cells were treated with 1.25, 2.5, 5, 10, and $20 \mathrm{nM}$ PTX or PVP/PTX-SAs in order to determine the cell proliferation capability. Cell lines with no treatment served as controls, respectively. After 48 hours of treatment, $20 \mu 1$ of MTS reagent solution was added to the cells and incubated for 2 hours at $37^{\circ} \mathrm{C}$. Absorbance reading was recorded at $490 \mathrm{~nm}$ using a Microplate Reader (BioTeK 
Cytation 3, Winooski, VT, USA). Data were plotted using cell proliferation against the concentration of treatment, where the proliferation of PVP/PTX-SAs and PTX was determined by the percentage of the absorbance of treated cells to the absorbance of control or non-treated cells. All the experiments were performed at least in triplicates.

\section{Clonogenic assay}

Clonogenic assay was performed to investigate the effect of PVP/PTX-SAs on BC cells to form colonies as an in vitro cell survival assay based on the ability of a single cell to grow into a colony. For this study, BC cells were seeded (250 cells/well) in a 12-well plate and allowed 2 days to attach and start generating smaller colonies. Then, these initiated cell colonies were treated with $0.25,0.5$ and $1 \mathrm{nM}$ PTX and PVP/PTX-SAs for two weeks. Visible colonies were fixed, stained with hematoxylin and manually counted ( $\sim 50$ cells considered as a colony) as discussed earlier $[261,266]$. The results are presented as the percentage of colonies as compared to the control (non-treated cells).

\section{$\beta$-Tubulin Stabilization Assay}

$\beta$-tubulin stabilization assay was performed by immunofluorescence assay to examine PTX inherent tubulin binding and alteration efficacy of PVP/PTX-SAs [261]. Briefly, 25,000 cells/well were cultured in 4-well chamber slides and cultured overnight to attach to a glass slide before treatment with $5 \mathrm{nM}$ PTX or PVP/PTX-SAs or respective controls (DMSO or polymer) for 8 hours. After treatment, cells were rinsed with 1X PBS for 3 mins three times, fixed with ice-cold methanol for 20 mins, blocked with 10\% goat serum for 1 hour. Then, cells were immunostained overnight with $\beta$-tubulin antibody (1:50 cell signaling, $\# \mathrm{CS} 2146)$ at $4{ }^{\circ} \mathrm{C}$ on a rocker with 20 oscillations/min. After incubation with the primary antibody, cells were rinsed three times with 1X PBS then probed with Alexa Fluor 488 goat anti-rabbit secondary antibody (1:200, \# A11008, Thermo Fisher Scientific, Waltham, USA) for 1 hour. DAPI was used to counterstain nuclei of cells. Finally, the polymerization of tubulin in cells was visualized under a laser confocal microscope (Carl Zeiss LSM 710, Thornwood, NY, USA) with a $40 \times 0.7 \mathrm{NA}$ oil immersion objective.

\section{Immunoblot Analysis}

For immunoblot analysis, BC cells $\left(1 \times 10^{6}\right.$ in $10 \mathrm{ml}$ medium $)$ were seeded in a $100 \mathrm{~mm}$ culture dish and treated with $10 \mathrm{nM}$ PTX or PVP/PTX-SAs or respective control (DMSO and PVP) for 48 hours. These cells were lysed with Cell Lytic ${ }^{\mathrm{TM}} \mathrm{M}$ reagent (Sigma-Aldrich Co. (St. Louis, MO, USA), the protein concentrations were measured, and equal concentrations of total protein were separated by SDS-PAGE, and the Western blotting methods were followed as described previously [261, 262]. Primary antibodies [ $\beta$-actin (\#4967), Bax (\#2772), BID (\#2003), cleaved caspase7 (\#9491), Bcl-xL (\#2762), cleaved PARP (\#9548) and MDR1/ABCB1 (\#13342)] (Cell Signaling Technologies, Danvers, MA) and secondary antibody (horseradish peroxidase-conjugated goat anti-mouse \#7076P2 or goat anti-rabbit \#7074P2 secondary antibody) (Cell Signaling) were used in this study. The specific protein bands were detected 
using enhanced Lumi-Light Detection Kit reagent under a Biorad ChemiDoc ${ }^{\mathrm{TM}}$ MP System (Biorad, Hercules, CA, USA).

\section{Statistical Analysis}

All statistical analyses were performed with GraphPad Prism 5 Software (GraphPad Software, San Diego, CA) using Student's t test. The difference was considered to be significant for $p$ values of $<0.05$. DLS (particle size and zeta potential), hemolysis, cellular uptake, and MTS assay data were expressed as mean \pm standard error of the mean. All graphs were generated using GraphPad Prism 5 Software.

\section{Results}

\section{Development of a Suitable Poly/PTX-SA Formulation for BC}

A successful self-assembled nanoparticle formulation may deliver a therapeutic agent at the tumor site more efficiently than the parent PTXPTX drug molecule alone. The selection of such a self-assembly carrier depends on its physio-chemical and biological characteristics. Therefore, in this investigation, we used eight commonly used pharmaceutical delivery vehicles to load PTX by the self-assembly technique which allows the formation of nanoparticles. The inherent thermodynamic forces direct the nanoparticle formation without the use of external agents. A similar self-assembly process was studied, described and reported by our group for $\beta$ cyclodextrin-curcumin and poly( $\beta$-cyclodextrin)-curcumin assemblies $[263,264]$. These selfassembled nanoparticles are much more stabilized and thus prevent aggregation and flocculation. To further ensure that solution is homogeneous we have probed sonicated the self-assembled nanoparticles, this warrants that there is no agglomeration occurring [265]. The formed selfassemblies of PTX with various polymers produced particle sizes ranging from $140.53 \mathrm{~nm}$ to $358.86 \mathrm{~nm}$ which was measured by dynamic light scattering measurements (Figure 2-1A). A lower particle size self-assembly was observed with PVP/PTX-SAs $(140.53 \pm 7.08 \mathrm{~nm})$ and Povacoat F-PTX-SAs $(145.2+5.33 \mathrm{~nm})$ due to strong PTX holding capacity within their polymer chain networks. High positive zeta potential formulation often induces systemic toxicity. In zeta potential measurements, most of the Poly/PTX-SA exhibited negative zeta potential (between $4.34 \mathrm{mV}$ to $-13.1 \mathrm{mV}$ ) (Figure 2-1B) while PA-HCl $(+9.54 \mathrm{mV})$ and PEI $(+3.09 \mathrm{mV})$ exhibited positive zeta potential.

To further screen all these Poly/PTX-SAs for cancer therapeutics, a suitable selfassembly formulation must be hemocompatible. This property is highly recommended for any clinically applied or approved formulation. The safety profile of PTX-SAs on RBCs for hemocompatibility was examined at $10 \mu \mathrm{g} /$ well of PTX or Poly/PTX-SAs. Results suggest that PVP, PVA, PA-HCl, and Poloxamer 188 based PTX-SAs exhibited negligible hemolysis (similar to the negative control treatment, PBS) while other PTX-SAs demonstrated higher hemolysis (more than 20\% hemolysis) like a free PTX drug (Figure 2-2A). This hemocompatibility 


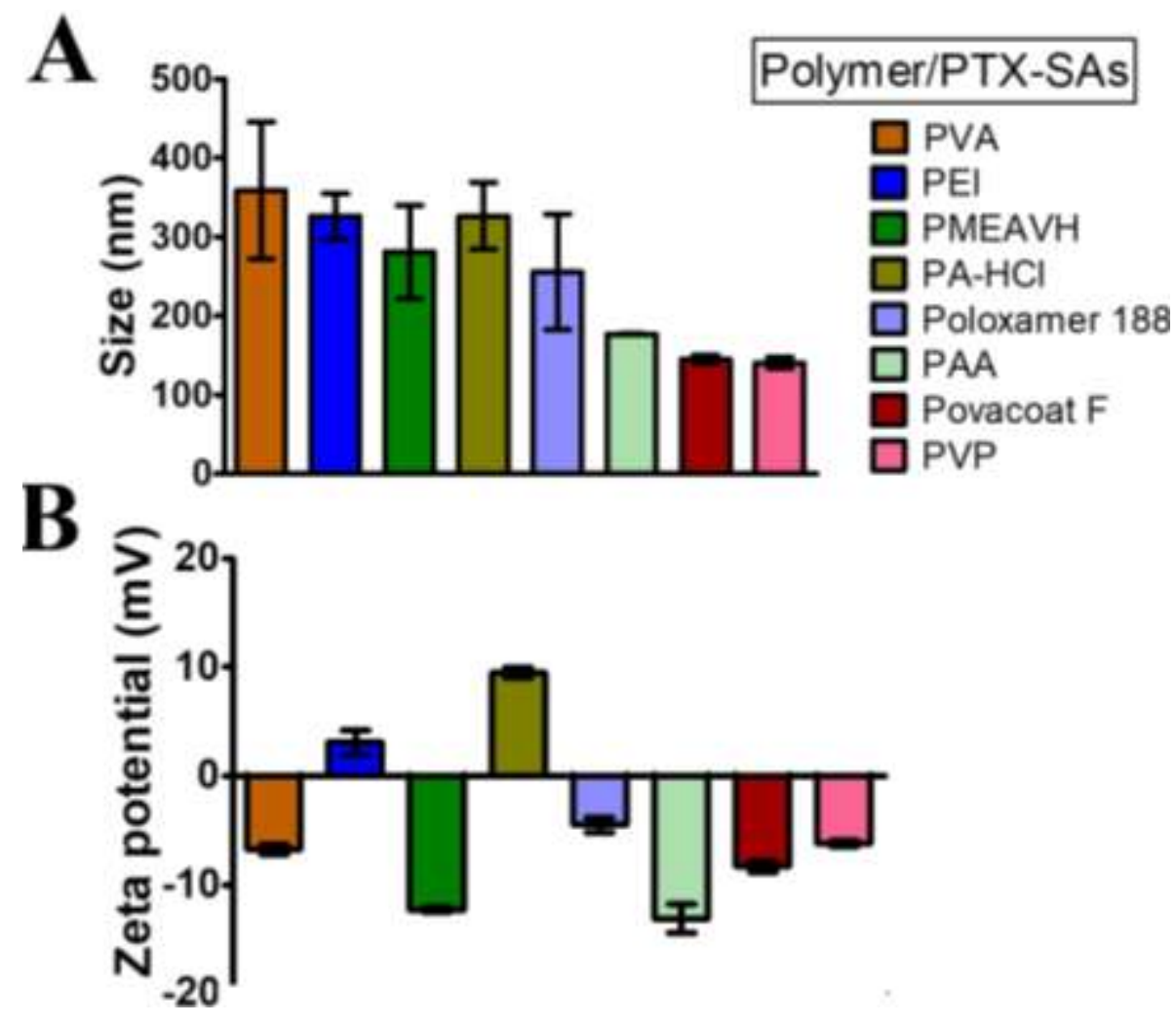

Figure 2-1. Particle size and zeta potential of Poly/PTX-SAs

$50 \mu \mathrm{l}$ of Poly/PTX-SAs were dispersed in $1 \mathrm{ml}$ distilled water or 1 X PBS and probe sonicated for 30 secs. These solutions were measured for particle size and zeta potential using dynamic light scattering at $25^{\circ} \mathrm{C}$ using Zetasizer (Nano ZS, Malvern Instruments, Malvern, UK). A) Average particle size of polymer PTX-SAs was measured for 3 min exhibiting the smallest size for PVP/PTX-SAs (140.53 nm). B) Zeta potential of Poly/PTX-SAs was measured in 1X PBS and average of 3 readings (each reading $=30$ runs) were presented. Data represented as an average of three readings that were recorded independently with error bars represented as standard error of mean. 

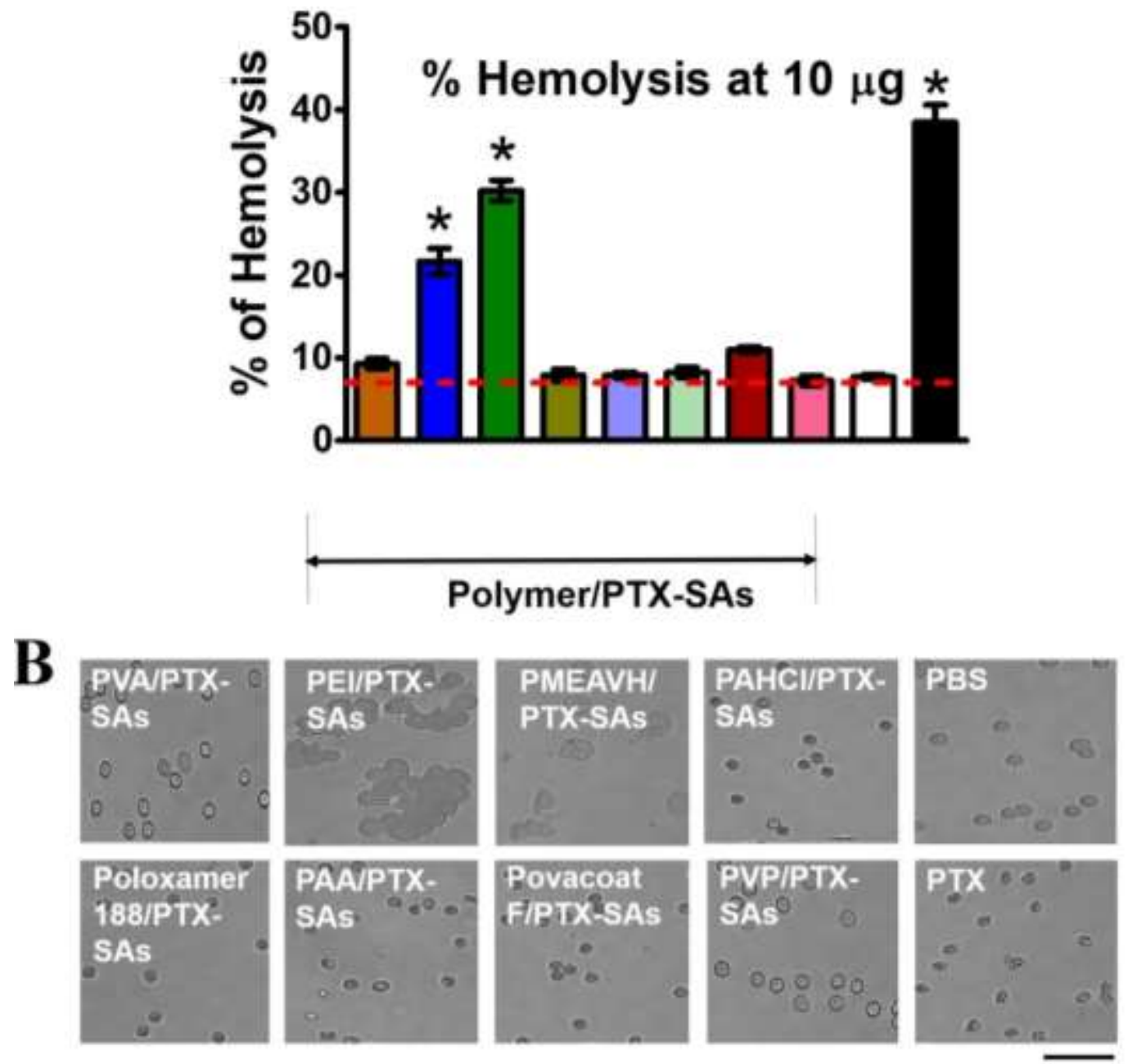

$25 \mu \mathrm{m}$

Figure 2-2. Evaluation of hemocompatibility behavior of Poly/PTX-SAs

A) Hemocompatibility was assessed using human red blood cells by treating them for $1 \mathrm{hr}$ with PTX or Poly/PTX-SAs. In this experiment, sodium dodecyl sulfate (SDS, $1 \mathrm{mg} / \mathrm{ml}$ ) served as a positive control (100\% lysis) while $1 \times$ PBS was used as a negative control. The percentage of hemolysis was calculated using our previously reported method. B) Images of treated red blood cells on glass slide were captured using EVOS ${ }^{\circledR}$ FL Imaging System (AMF4300, Life Technologies, Carlsbad, CA, USA). PVP/PVA-PTX-SAs demonstrate intact membrane morphology in RBCs as PBS, whereas PTX and other Poly/PTX-SAs exhibited toxic behavior by disintegrating membrane structure of RBCs. 
behavior with PVP and PVA is correlated with intact membrane morphology in RBCs just like a negative control (PBS) treated RBCs (Figure 2-2B). All other Poly/PTX-SAs exhibited severe influence on shape and morphology of RBCs (Figure 2-2B). PTX alone exhibited significant toxic behavior by disintegrating membrane structure of RBCs.

Irrespective of the type of drug formulation, they must interact and penetrate through the cell membrane of cancer cells, then be released into the cytosol at sufficient concentration to induce therapeutic benefit. The therapeutic outcomes are improved if the intracellular uptake of nanoparticle is improved. Thus, higher internalization of formulation in the cells is the key factor to determine the efficiency of the nanoparticle formulation. The cellular uptake screening was conducted using dye loaded polymer assemblies which can be detected by green fluorescence in cells which directly reflects the internalization of the formulation. This study demonstrates an uptake of C6 pattern in MDA-MB-231 cells as PVP $>$ PVA $>$ PMEAVH $>$ Povacoat F $>$ PAHCL $>$ PEI > Poloxamer $188>$ PAA PTX-SAs, whereas similar pattern was observed in MCF7 cells with the highest cellular uptake in PVP/PTX-SA (Figure 2-3A). The mean fluorescence intensities of PVP/PTX-SAs and PVA/PTX-SA were 16 and 14; 10 and 9 times higher in MDA-MB-231 and MCF7, respectively (Figure 2-3A). Fluorescence images of cells is further evidence for similar uptake characteristics of polymer self-assemblies than that observed in flow cytometry. PVP and PVA self-assemblies were detected in cancer cells all over the cellular organelles while other polymers show less fluorescence in these cells (Figure 2-3 B). However, the higher size range of PVA/PTX-SA of $358.86 \mathrm{~nm}$, minimized the chances of using it as a potential nanoformulation. Thus, we selected PVP/PTX-SAs for the rest of the study, as PVP is a better binder for transporting loaded therapeutics into cancer cells. From the DLS, hemocompatibility, and cellular uptake studies, it can be reported that PVP/PTX self-assemblies have the optimal size and negative charge while maintaining hemocompatibility and improved cellular uptake in cancer cells that are may be helpful for cancer therapeutic purposes. Thus, all our further studies in this manuscript are focused on PVP/PTX-SAs.

\section{Characterization of PVP/PTX SA Formation}

To confirm that PVP and PTX form self-assembly nanoparticles, we utilized PTX conjugated dye (PTX, Oregon Green 488 conjugate) for fluorescence quenching assay. In the first method, to confirm the efficient binding of PTX with the PVP, a quenching study was performed against PTX conjugated dye (PTX, Oregon Green 488 conjugate) in 1X PBS solution (Figure 2-4A). Fluorescence quenching reveals possible interaction of the polymer with PTX dye. This is an instantaneous process directly couples/self-assembles PTX to the polymer. Such instantaneous particle formation and integrity was confirmed by hydrodynamic diameter measurements using DLS. The fluorescence intensity of PTX dye was diminished with the increase of PVP solution. This directly correlates to the binding potential of PVP with PTX which indicates the successful formation of self-assemblies. The inclusion of PVP and PTX in PVP/PTX-SAs can be confirmed using FTIR analysis. The acquired FTIR spectra of the PVP, PTX, and PVP/PTX-SAs (Figure 2-4B) demonstrate that PTX has distinct visible peaks at 1734 $\mathrm{cm}^{-1}, 1704 \mathrm{~cm}^{-1}$ and $1645 \mathrm{~cm}^{-1}$ (due to $\mathrm{C}=\mathrm{O}$ stretching of amide and ester functionality) which were not visible in PVP/PTX-SA thus suggesting a possible interaction of PVP with PTX through these functional groups 

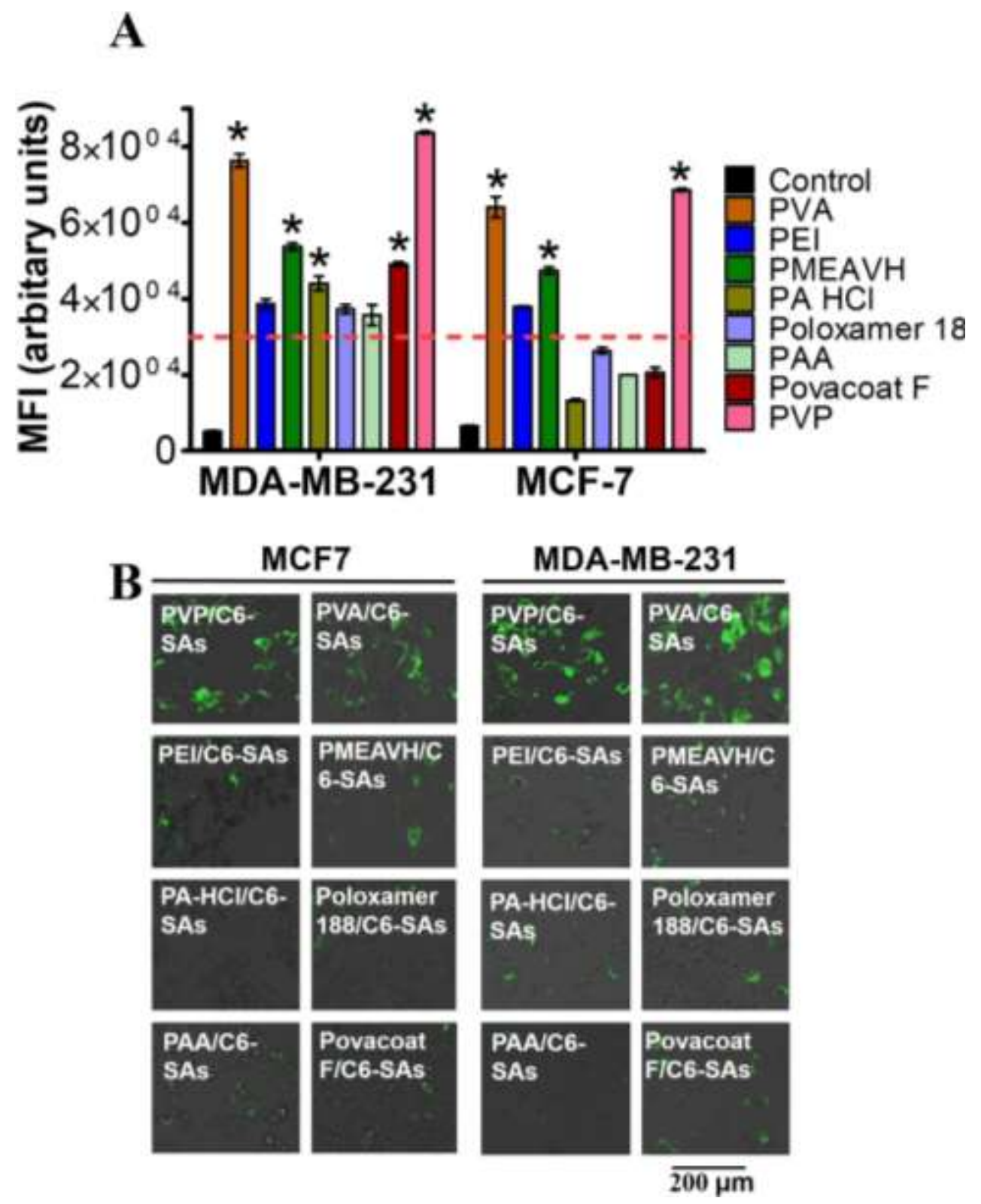

Figure 2-3. Cellular uptake of Poly/PTX-SAs

A) Quantitative cellular uptake evaluation All the cells were treated with Poly SAs loaded with C6 instead of PTX in order to evaluate fluorescent uptake in cells. BC cells $(500,000)$ were treated with Poly/C6-SAs (5 $\mu$ g dye equivalent formulations) for 3 hours. These cells were trypsinized, centrifuged and collected in $2 \mathrm{ml}$ media, which were injected ( $50 \mu \mathrm{l}$ cell suspension) into an Accuri C6 Flow Cytometer (Accuri Cytometer, Inc., Ann Arbor, MI, USA). Mean fluorescence intensity in FL1 channel (488 excitation, Blue laser, $530 \pm 15 \mathrm{~nm}, \mathrm{FITC} / \mathrm{GFP}$ ) was measured. Data represented as mean \pm standard error of the mean $(\mathrm{n}=3),{ }^{*} \mathrm{P}<0.05$. B) Qualitative internalization efficiency of Poly/C6-SAs in BC cells. In above treatment condition, uptake of Poly/Couamrin 6-SAs in BC cells were viewed using EVOS ${ }^{\circledR}$ FL Imaging System (AMF4300, Life Technologies, Carlsbad, CA, USA). Bar equals to $200 \mu \mathrm{m}$. This study suggests fluorescence intensity in the order: $\mathrm{PVP}>\mathrm{PVA}>\mathrm{PMEAVH}>$ Povacoat $\mathrm{F}>\mathrm{PA}-\mathrm{HCL}>\mathrm{PEI}>$ Poloxamer 188 > PAA C6-SAs. 


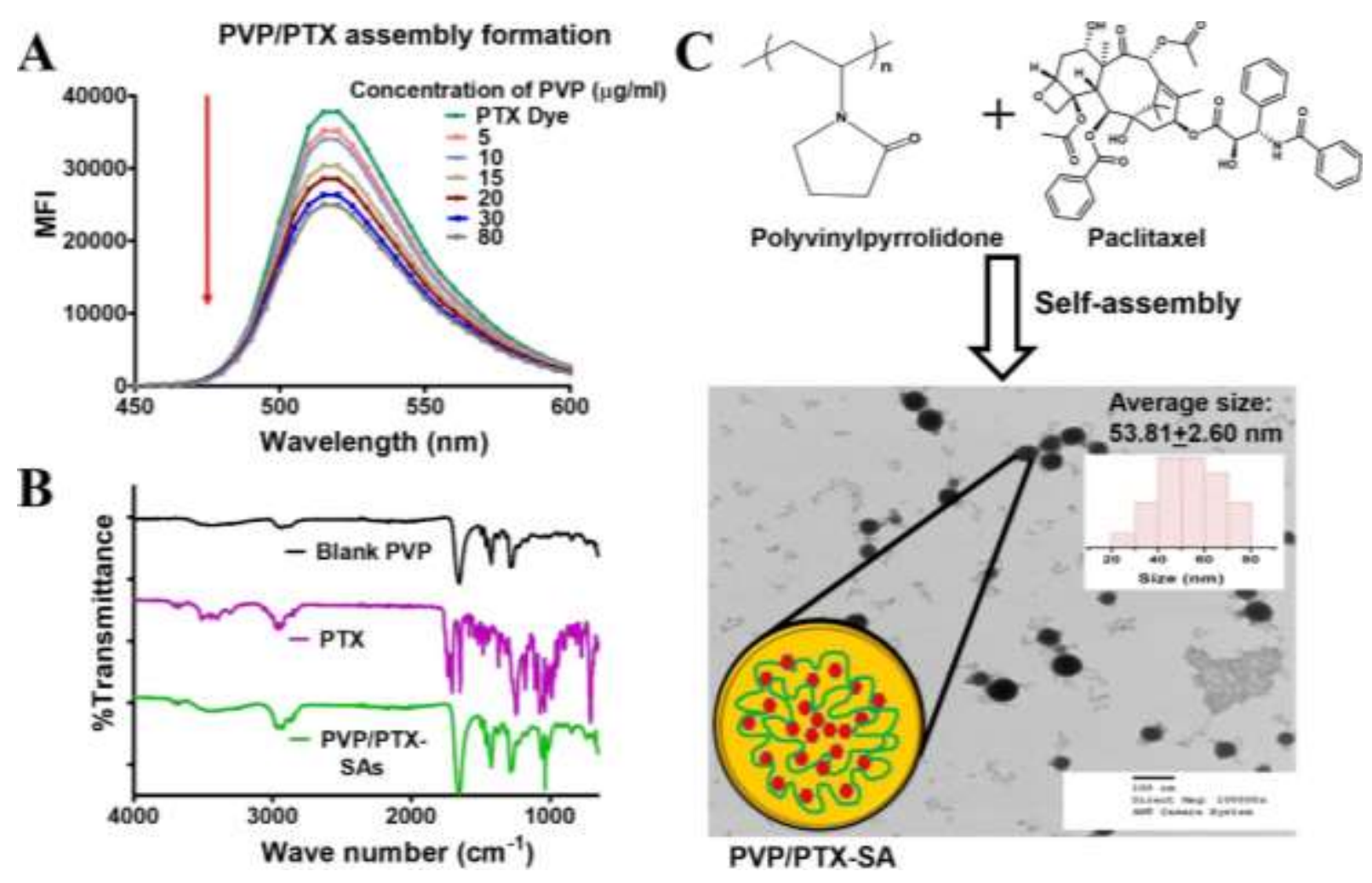

Figure 2-4. Self-assembly formation of PVP/PTX-SAs

A) Self-assembly formation was confirmed by binding potential (quenching) of PTX conjugated dye (PTX, Oregon Green 488 conjugate (Flutax-2, Molecular Probes Inc., Life Technology, OR, USA)) with PVP solution $(5-80 \mu \mathrm{g} / \mathrm{ml})$. Fluorescence was studied by exciting at $496 \mathrm{~nm}$ and emission between 450-600 nm. B) FTIR spectra of PVP/PTX-SAs, PTX, and PVP. The presence of $1033 \mathrm{~cm}-1$ (characteristic PTX peak) and 1270, 1284, 1420 and $1654 \mathrm{~cm}-1$ characteristic PVP peak) confirms the presence of both PVP and PTX in the self-assembly formation of PVP/PTXSAs. C) Schematic and hypothetical structural presentation of PVP/PTX-SAs. Color, dimensions, assembly are not comparable to synthesized formulation. Morphology of PVP/PTXSAs imaged by using AMT camera at a direct magnification of 100,000x under the TEM on a 150 -mesh standard TEM grid, stained by $2 \% \mathrm{w} / \mathrm{v}$ uranyl acetate solution. The particle size viewed under TEM ranged between 34.88 to $130.24 \mathrm{~nm}$. 
Further, in the PVP/PTX-SAs spectrum, the intensity of the broad band around $3259 \mathrm{~cm}^{-1}$ was reduced suggesting another possible interaction motif with the alcoholic O-H functionality too. If we look into the fingerprint region of the FTIR spectrum for the PVP/PTX-SAs formulation, the sharp peak at $1033 \mathrm{~cm}^{-1}$ confirms the presence of PTX in the formulation. Lastly, the peaks at 1270, 1284, 1420 and $1654 \mathrm{~cm}^{-1}$ of both PVP/PTX-SA and PTX substantiates the coexistence of both PVP and PTX in the given formulation. The FTIR study was performed to confirm the existence of polymer and PTXPTX in its formulation but not to verify the course of selfassembly formation between polymer and PTXPTX. Additional evidence is to examine its nanoparticle formation using transmission electron microscopy (TEM) for the size and the morphology of self-assemblies (Figure 2-4C). A clear contrast of self-assembled nanoparticles formation was observed with positively stained ( $2 \% \mathrm{w} / \mathrm{v}$ uranyl acetate solution) PVP/PTX-SAs. From this study, it is evident that the average size of the dry and stained PVP/PTX-SAs found to be $53.81 \pm 2.6 \mathrm{~nm}$ (Note: DLS data provides particles in suspension or wet form which always exhibit higher particle size over TEM measurements). This nanoparticle observation substantiates and affirms the hydrodynamic size of the nanoparticles measured by DLS.

\section{In Vitro Anticancer Potential of PVP/PTX-SAs}

The therapeutic efficacy of PVP/PTX-SAs was evaluated in BC cell lines by MTS viability and colony formation assay [263]. Both PTX and PVP/PTX-SAs showed a dosedependent antiproliferation effect (1.25-20 nM) in MCF7 and MDA-MB-231 (Figure 2-5A). The control polymer did not show any effect on cell growth. The in vitro $50 \%$ cell growth inhibitory concentration $\left(\mathrm{IC}_{50}\right)$ is the quantitative measure of the toxicity induced by the treatment group on the cells. $\mathrm{IC}_{50}$ values for PTX is $13.12 \mathrm{nM}$ and 12.40 whereas $\mathrm{IC}_{50}$ for PVP/PTX-SAs is 4.68 and $7.26 \mathrm{nM}$, for MCF7 and MDA-MB-231, respectively. Similarly, images of treated cells indicate that the inherent anticancer activity of PTX is preserved in PVP/PTX-SAs (Figure 2-5B). PVP/PTX-SA formulation is more potent in suppressing cell growth compared to PTX. A similar superior active performance of PVP/PTX-SAs was achieved in the colony forming assay, which is another complementary assay to evaluate anticancer efficacy (Figure 2-6). In this study, PVP/PTX-SAs have shown a significant decrease in colonies and colony density over PTX for both MCF7 and MDA-MB-231 (Figure 2-6A, B). Such high anti-proliferative and colonogenic potency of PVP/PTX-SAs at a low effective dose compared to PTXPTX demonstrates that a lesser concentration of PTX is needed in self-assembly formulations for effective therapeutic outcomes.

\section{Molecular Activity of PVP/PTX-SAs}

At the cellular front, PTX binds to the $\beta$-tubulin subunits in microtubules and induces polymerization of tubulin which disrupts microtubule dynamics, giving rise to mitotic arrest and thus cell death [266]. To obtain more insight in this aspect of PVP/PTX-SAs, we performed $\beta$ tubulin stabilization using a specific antibody in immunostaining and imaging by confocal microscopy. A higher microtubule polymer mass was observed on treatment with PVP/PTXSAs, in contrast with PTX (5 and $10 \mathrm{nM}$ ) or respective control groups (DMSO or PVP). Higher microtubule bundles in cells, suggested by the green fluorescence observed for microtubule 

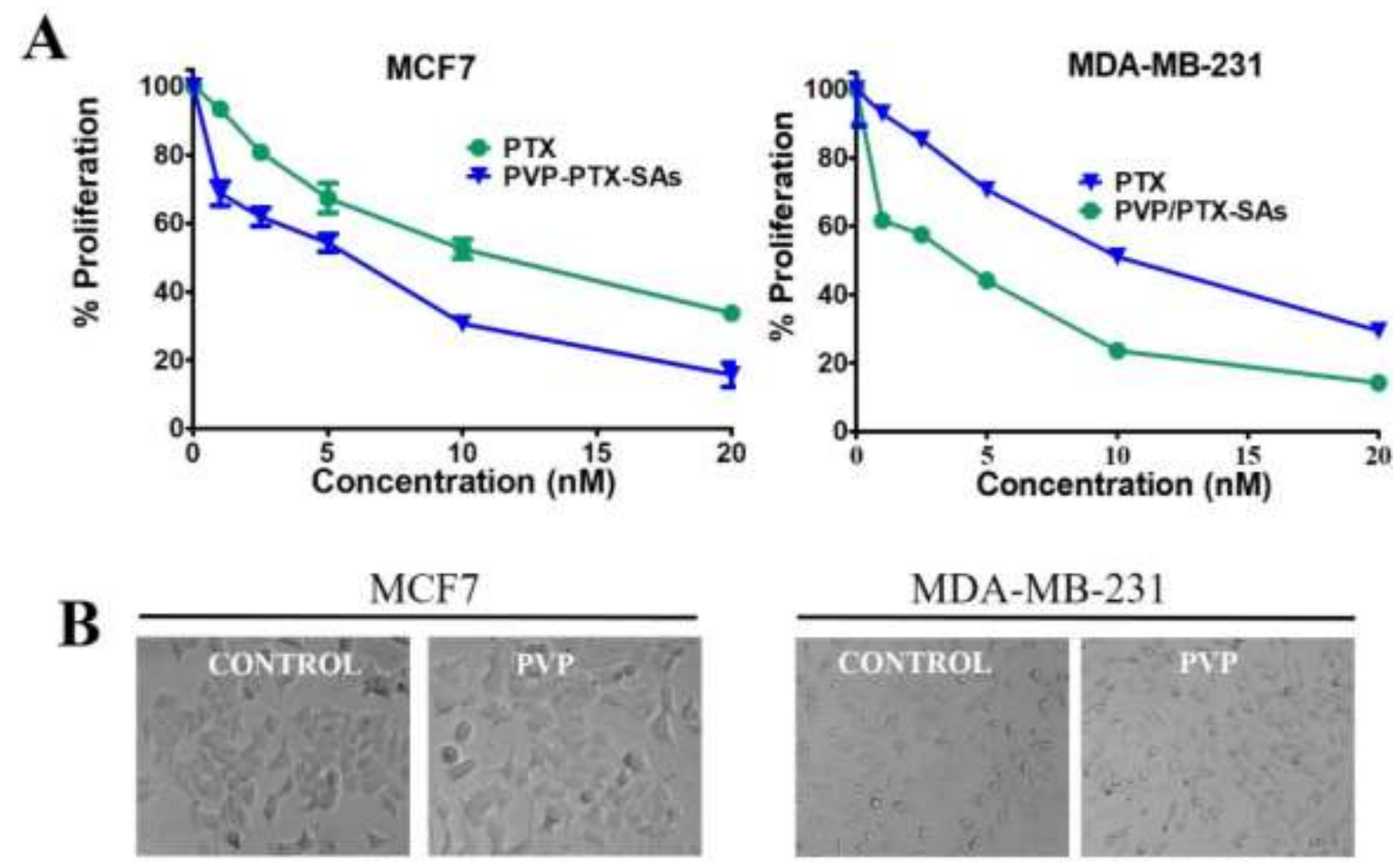

MCF7
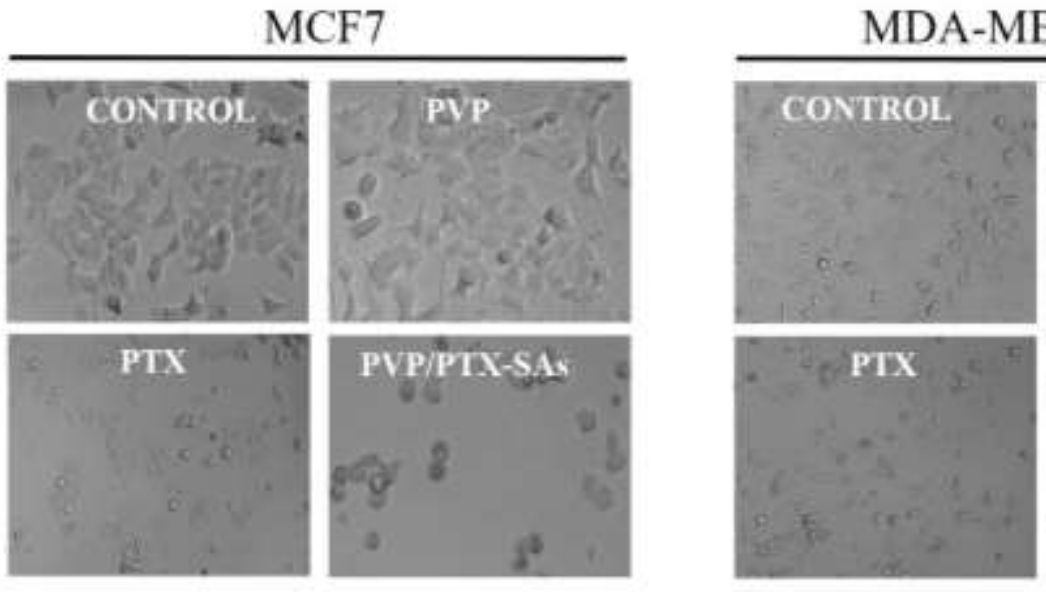

$\overline{200 \mathrm{um}}$

Figure 2-5. PVP/PTX-SAs significantly inhibit proliferation of $B C$ cells

A) BC cells (MCF7 and MDA-MB-231) (5000 cells/well) were plated in 96-well plates and were treated with PTX or PVP/PTX-SA at a range of concentration 1 to $20 \mathrm{nM}$ for 48 hours. Cell viability was assessed by MTS assay by measuring absorbance at $490 \mathrm{~nm}$ using microplate reader., Relative cell viability was measured (using GraphPad Prism 5 Software) with control (untreated) BC cells, considering as $100 \%$. Data represented as mean \pm standard error of the mean $(\mathrm{n}=3), \mathrm{B})$ Representative phase-contrast microscopic images were captured by EVOS ${ }^{\circledR}$ FL Imaging System to evaluate the effect of PVP/PTX-SAs on the cell morphology. Bar equals to $200 \mu \mathrm{m}$. 

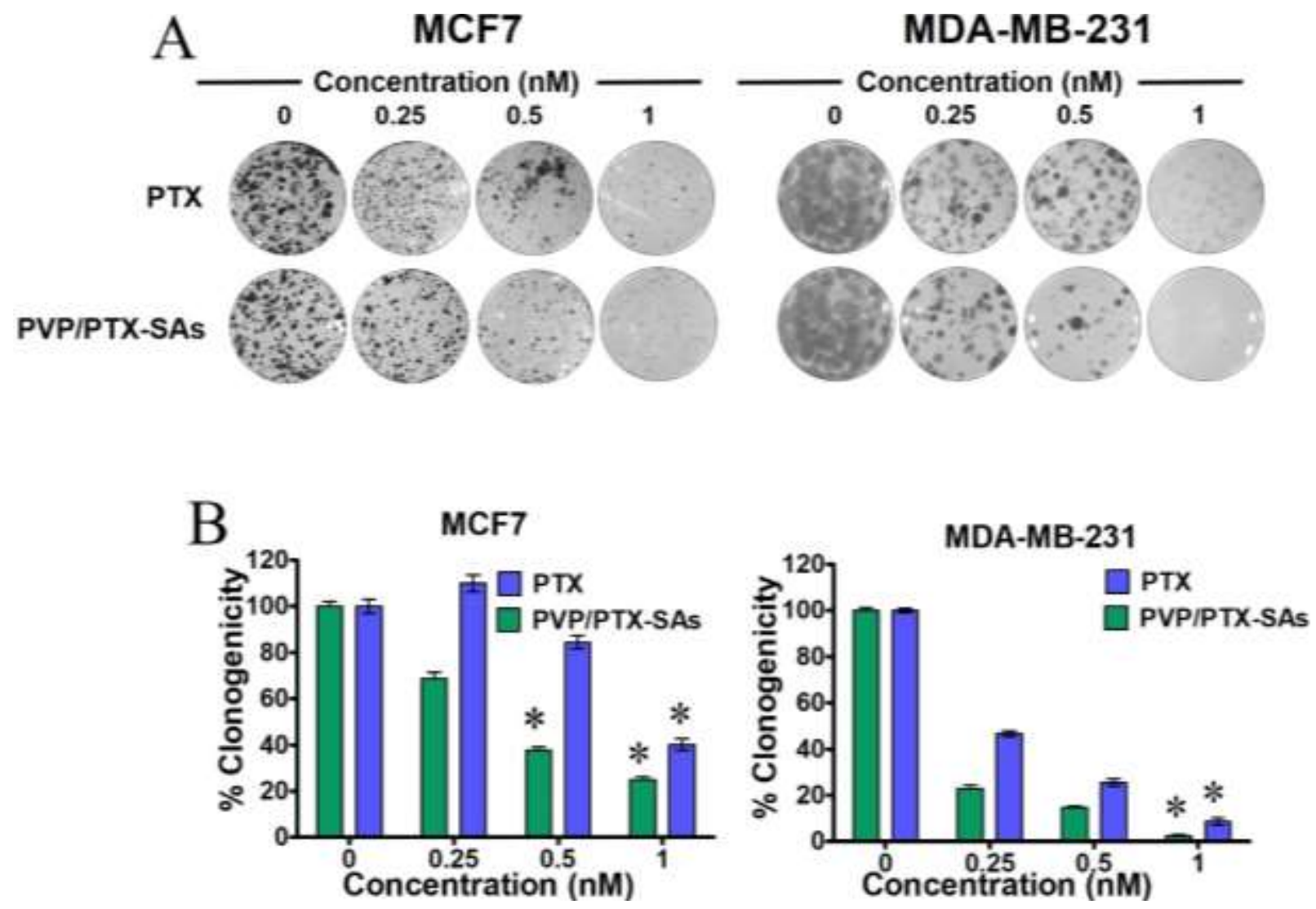

Figure 2-6. $\quad$ PVP/PTX-SAs repress the clonogenic potential of $\mathrm{BC}$ cells A) BC cells (MCF7 and MDA-MB-231) (250 cells/well in a 12-well plate) were treated with 0.25, 0.5, and $1 \mathrm{nM} \mathrm{PVP/PTX-SAs} \mathrm{and} \mathrm{PTX} \mathrm{along} \mathrm{with} \mathrm{their} \mathrm{respective} \mathrm{controls.} \mathrm{On} \mathrm{day} \mathrm{14,}$ cells were rinsed with PBS and stained with hematoxylin to visualize colonies for counting. Photographs of clonogenic pattern were captured in Multimage ${ }^{\mathrm{TM}}$ light cabinet. B) Bar graphs represent number of colonies formed in each treatment group. Data represented as mean \pm standard error of the mean $(n=3)$. 
staining (Figure 2-7A), is due to the enhanced antitumor activity of PVP/PTX-SAs compared to PTX. This behavior was more prominent even at $10 \mathrm{nM}$ (Data not shown) concentration with PVP/PTX-SAs indicating the use of PVP/PTX-SAs may reduce concentrations required to induce the anticancer effect.

We performed immunoblot analysis to further understand the superior anticancer function of PVP/PTX-SAs in BC cells. Expression of various proteins such as Bcl-xL, Bid, Bax, Cleaved PARP, Cleaved Caspase 7, and MDR1 upon treatment with PTX and PVP/PTX-SAs was assessed (Figure 2-7B). The expression of the key pro-survival protein (Bcl-xL) was effectively down-regulated by PTX and PVP/PTX-SAs treatment. Also, expression of pro-apoptosis proteins (Bax, Bid), Cleaved PARP, and Cleaved Caspase 7, increased following PTX and PVP/PTX-SAs treatment. These events are more prominent in PVP/PTX-SAs strongly suggests PVP/PTX-SAs induce apoptosis more pronouncedly in comparison to the native drug PTX [267]. Further, the effect of treatment on MDR1 protein expression was also observed. Altogether, we can conclude that PVP/PTX-SAs were able to stabilize tubulin polymerization and cause intrinsic differences in molecular effects, which could effectively induce apoptosis.

\section{Discussion}

The main problem of PTX-based chemotherapy is severe systemic toxicity due to the nonspecific accumulation of anticancer agents. In other words, the administration of PTX with the use of lipophilic solvents or surfactant(s) may cause insufficient drug levels at the tumor site or quick clearance due to the aggregation of PTXPTX nanoformulation. Although scientific literature and clinical evidence suggest some formulations have exhibited superior therapeutic outcomes, a simple preparative approach like self-assembly formulations of PTX is preferred $[268,269]$. Therefore, the main focus of this study is to develop PTX/polymer-based nanoformulations by self- assembly technique to enhance the efficacy of PTX in BC treatment. Polymeric nanoparticles are a well-known alternative in the field of drug delivery for therapeutic benefits.

In this same regard, we have prepared nanoparticles which are colloidal systems with PTX entrapped in the matrix. The unique structure of the biodegradable polymeric matrix provides advantages of target-specific delivery and intracellular penetration. Thus achieving sustained drug release, preventing frequent administration and an overall broad spectrum of therapeutic benefits are expected [270]. The unique preparative approach of the self-assembly technique enables formulation scientists to reduce the usage of external excipients and in the process reduces the chances of toxicity from the nanocarrier itself. It is reported that the superiority of self-assembled nanoparticles in the field of drug delivery is favored [268, 269]. The hydrophilic aqueous polymeric solution serves as a bed for the hydrophobic drug through van der Waals forces/interaction or hydrogen bonding at the core of the particle. Hydrophobic interaction between the hydrophobic polyvinyl group of PVP and PTX is responsible for the formation of the nanoformulation. Due to steric stabilization, these nano-drug formulations have longer retention in the bloodstream whereas their nanosized structures facilitate the nanoformulation to escape through the leaky vasculature and release of drug at the tumor sites owing to their enhanced permeability and retention effect [271]. Self-assembled drug 


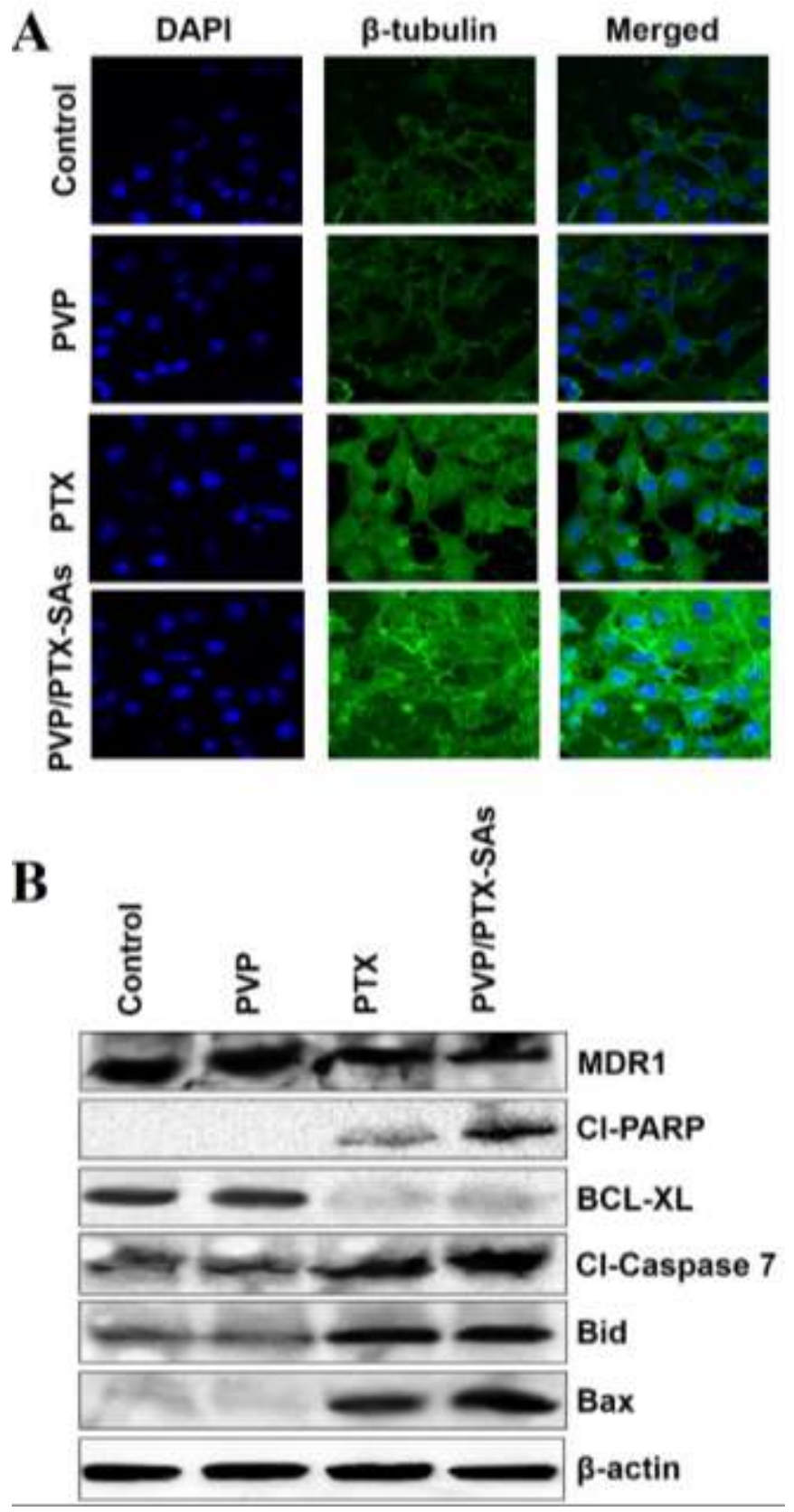

Figure 2-7. Preferential cell death induced by PVP/PTX-SAs in MDA-MB-231 cells A) Tubulin stabilization images of PVP/PTX Self Assembly. Effects of PVP/PTX-SA and PTX through PTX-induced microtubule stabilization by mitotic arrest leading to cell death. Cells have been treated with equivalent concentrations of $5 \mathrm{nM}$ PTX or PVP/PTX-SA for $8 \mathrm{hr}$. Imaged by Carl Zeiss LSM 710. Original magnification 400X. B) MDA-MB-231 cells were treated with 10 nM PVP/PTX-SAs or PTX or respective controls for 48 hours. Cell lysates were analyzed to detect levels of Bax, Bid, Cleaved Caspase 7, Cleaved PARP, Bcl-xL, and MDR1, with $\beta$-actin as a control. The results were consistent in two independent sets of experiments. 
Self-assembled drug nanoparticles have gained significant interest in the therapeutic field due to biocompatibility [272], biodegradability, and low immunogenic properties [273, 274].

In this study, we have examined the ability of eight commonly used pharmaceutical polymers to generate Poly/PTX-SAs. Among these eight polymers, PVP polymer not only was capable of producing the smallest size $(140.53 \mathrm{~nm})$ with negative zeta potential $(-6.21 \mathrm{mV})$ (Figure 2-1) but exhibited lower toxicity with RBCs (Figure 2-2). This behavior is better than PTX alone. Additionally, these PVP-C6-SAs exhibit superior interaction with BC cells compared to other Poly/C6-SAs (Figure 2-3). The size and zeta potential properties of the PVP/PTX-SAs formulation resembles clinically used PTX nanoparticle formulations such as Abraxane ${ }^{\circledR}$ and Genexol ${ }^{\circledR}$ PM. A negative zeta potential is favorable for the stability of the particles, prevents them from binding to the plasma proteins and prolongs the circulation time of the nanoparticles [275]. Owing to such physicochemical characteristics of these nanoparticles of nanosized range and negative zeta potential, off-target delivery can be avoided. As it is the inherent nature of these nanoparticles, they tend to target at the tumor site [276]. The self-assembly formation of PVP and PTX was further confirmed by TEM (particle assembly leads to $53.81 \mathrm{~nm}$ ) and a continuous dose-dependent binding profile with PTX in fluorescence quenching experiments (Figure 2-4). From the spectral analysis it is confirmed that the presence of chemical functional/repeating units of PVP and PTX in PVP/PTX-SAs that are found in the particles, suggesting that the integrity of the polymer(s) and PTX is maintained in the formulation.

PVP is widely used as an inactive pharmaceutical excipient for binder/disperser, film former, flavoring liquid, and adhesive applications for multiple uses such as tablets, capsules, ophthalmic solutions, and transdermal patches. Earlier in 1950's, PVP was used as a plasma volume expander. More importantly, PVP is considered as a generally safe chemical for many uses by the FDA. Thus, it may hold promise in delivering PTX too. PVP was earlier implemented as a binder/stabilizer for PTX in a reverse microemulsion [277], solvent evaporation [278] and nanoprecipitation methods for PTX/PVP-PCL nanoparticles (110 nm) $[279,280]$. These studies support our current findings that PVP is a better choice for holding PTX in self-assembly grooves. PVP has been used as a solid dispersion for PTX and docetaxel $[281,282]$. Also, Sharma et al [277] also developed PVP nanoparticle-encapsulated Taxol ${ }^{\circledR}$. Our approach is direct self-assembly or solid dispersion method while the above formulation is based on the reverse microemulsion method. Based on our study, we believe that the self-assembly of PVP and PTX may happen due to co-precipitation.

To further utilize PVP as a self-assembly binder or delivery carrier for PTX, the nanoparticle must interact more closely with cancer cells and release effectively into the cytosol for maximizing the pharmacological effects of PTX. Through this study, we proved that the PVP/PTX-SAs induce a greater amount of formulation in cancer cells as observed with fluorescence microscopy and flow cytometry (Figure 2-3). Additionally, PVP/PTX-SAs exhibits superior anticancer activity in proliferation and clonogenic assays against BC cells, compared to PTX alone (Figures 2-5 and 2-6).

PTX is a classical and unquestioned microtubule inhibitor which exhibits clinical success. PTX promotes tubulin polymerization and stabilization of microtubules resulting in $\mathrm{G}_{2}-\mathrm{M}$ phase arrest and thus mitotic cell death occurs [283]. Development of improved tubulin-binding drugs 
or improving PTX tubulin-binding and improving the stability of microtubules' efficiency with formulations of any type are considered to be attractive in cancer therapeutics. In this investigation, we observed that our lead PVP/PTX-SAs are acting significantly on cell tubulin dynamics and tubulin polymerization, thus contributing to mitotic block in two BC cells (Figure 2-7A) [284]. This determines the impact of PVP's role in this formulation as a drug carrier. In cancer therapeutics, apoptosis induced by the Bcl-2 family is an important parameter as it allows prediction of the mitochondrial pathway [285]. In this regard, our study results showed distinct effects of PVP/PTX-SAs on apoptotic signaling with reduced expression of Bcl-xL, a profound expression of pro-apoptotic proteins (Bax and Bid), and the expression profile of Cleaved PARP, and Cleaved Caspase 7, in comparison to control and native drug treatments. This demonstrates that enhanced apoptotic cell death occurs with PVP/PTX-SAs treatment than with conventional exposure [286]. These results further affirm enhanced toxicity effects of prepared samples and are well consistent with the results of proliferation and clonogenic assays.

Altogether, PVP/PTX-SAs demonstrated that these nanoparticles are taken up by BC cells and induce superior anticancer activity in BC cells (Figure 2-8). Further, PVP is known to provide extensive stability to the therapeutic molecules and reduction of renal clearance [287]. These data support the feasibility of developing PVP/PTX-SAs as nanoparticle-based therapeutics for improving the therapeutic benefits of PTX. If needed further to generate this PVP/PTX-SAs targeted drug delivery technology, we have designed numerous experimental targeted delivery protocols $[258,259,261]$ that will be implemented to achieve higher therapeutic benefits of this self-assembly nanoparticle formulation through targeted approachability.

\section{Conclusion}

Introducing clinically relevant PTX (a hydrophobic anticancer drug) into self-assembled nanoparticles was successfully achieved. Based on particle size, cellular uptake, and hemolysis assays, PVP/PTX-SAs were found to be better formulations over seven other Poly/PTX-SAs. We observe superior anticancer effects of PVP/PTX-SAs through inhibition of proliferation and colony formation, which were further confirmed by tubulin stabilization and immunoblot analysis. These findings highlight the advantages of the implementation of PVP/PTX-SAs in comparison to other conventional PTX formulations. Although this approach led us to develop nanoparticles that showed better efficacy in comparison to conventional Taxol ${ }^{\circledR}$, yet their real time benefits need to be warranted in other in vitro and in vivo models to gain mechanistic insights. 


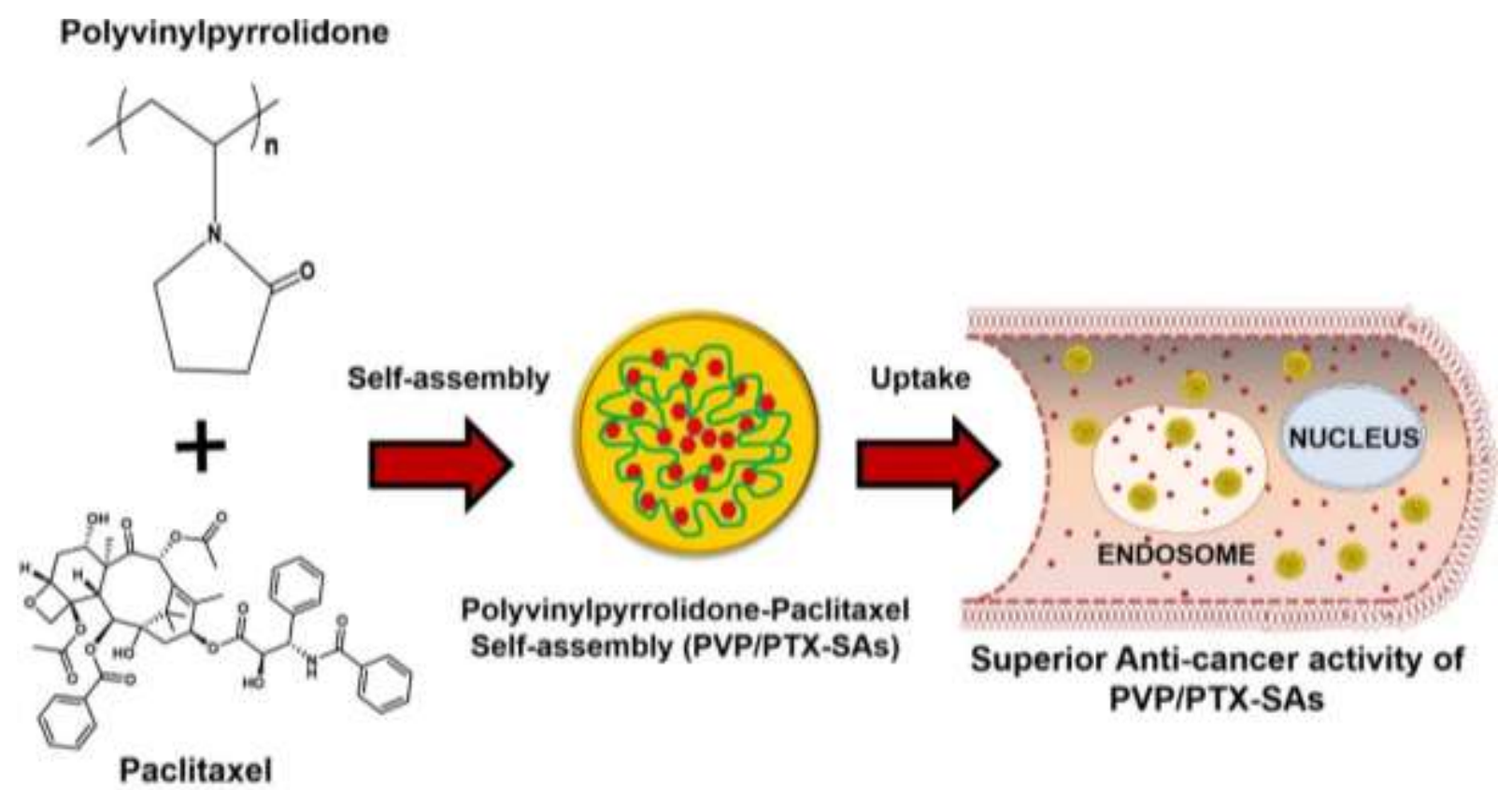

Figure 2-8. Schematic and hypothetical structural representation of PVP/PTX-SAs Color, dimensions and assembly are not comparable to synthesized formulation. 


\section{CHAPTER 3. TANNIC ACID-INSPIRED PTX NANOPARTICLES FOR ENHANCED ANTICANCER EFFECTS IN BC CELLS ${ }^{2}$}

\section{Introduction}

$\mathrm{BC}$ is one of the most commonly diagnosed cancers in American women in the United States [288]. Currently, 1 in 8 women (about 12\%) may develop BC in their lifetime. American Cancer Society 2018 statistics projected that about 266,120 new BC diagnoses and 40,920 deaths will occur due to this disease [288]. Apart from hormonal therapy, chemotherapy is the most effective treatment for $\mathrm{BC}$. Historically, the importance of taxane drugs in $\mathrm{BC}$ is well established. PTX is a taxane derived from the bark of Taxus brevifolia, first isolated and structure elucidated in 1971 [175]. The first generation pharmaceutical formulation $\left(\mathrm{Taxol}^{\circledR}\right)$ is well known for improving the overall survival rate and progression-free survival of patients [289, 290]. It was approved for the treatment of various cancers including BC. Despite the therapeutic benefits, the presence of Cremophor EL and ethanol in this formulation exerts serious adverse effects.

Various nanomedicine technologies have been put forth into the development of PTX delivery systems that not only increase the bioavailability of PTX, but also lower Taxol ${ }^{\circledR}$ associated systemic toxicities [291]. For instance, a clinically successful PTX nanoformulation is Nab-PTX, composed of solvent-free PTX protein-bound particles (Abraxane $\left.{ }^{\mathrm{TM}}\right)$. This formulation potentiates the selective uptake of PTX in tumors ( $\sim 33 \%$ greater tumor uptake vs. conventional PTX) and shows improved therapeutic index, lower systemic side effects, and avoids steroid premedication. Nab-paclitaxel was FDA-approved in 2005 for chemotherapeutic treatment of metastatic BC. Its later clinical trials led to FDA approval to treat non-small cell lung cancer (2012) and metastatic pancreatic cancer (2013). Similarly, many other PTX preclinical nanoformulations, notably Genexol PM ${ }^{\circledR}$, NK-105, Paclical ${ }^{\circledR}$, Lipusu $^{\circledR}$, PICN, SB05, LEP-ETU, and Triolimus, have received heightened attention in cancer treatment. However, many of these formulations have not made an impact similar to Nab-PTX. Many dietary polyphenolic compounds derived from plant sources are known to exhibit anti-cancer and chemosensitization properties. Strong scientific evidence suggests [292] that the incorporation of bioactive compounds in one's diet can often reduce the risk of cancer and other chronic diseases. These foods are consumed daily by humans and are thus non-toxic. Among many naturally occurring polyphenolic compounds, tannic acid has received much attention lately due to its excipient value in pharmaceutics, giving it medicinal value. Furthermore, TA has been employed in generating several biocompatible products for biomedical applications.

Tannic acid belongs to a subset of hydrolyzable tannin congeners commonly found in a variety of plants, fruits, grains, nuts, and tea leaves. Lately, TA has attracted attention as being an additive molecule, one that can provide improved solubilization of various hydrophobic drugs

\footnotetext{
${ }^{2}$ Modified from final submission with permission. Chowdhury, P., Nagesh, P. K., Hatami, E., Wagh, S., Dan, N., Tripathi, Khan, S., Hafeez, B. B., Meibohm B, Chauhan, S. C., Jaggi, M. \& Yallapu, M. M (2019). Tannic acidinspired paclitaxel nanoparticles for enhanced anticancer effects in breast cancer cells. Journal of colloid and interface science, 535, 133-148. https://doi.org/10.1016/j.jcis.2018.09.072
} 
(amphotericin B, curcumin, rapamycin, PTX, or docetaxel) for localized to parenteral applications. TA renders such additive features due to its low viscosity (easy for generating formulations), excellent water solubility (not all polyphenols exhibit water solubility), and biocompatibility. TA inherently possesses such desired nanocarrier properties primarily because it can bind to drug molecules via hydrophobic interactions, which in turn forms a self-assembled crosslinked network via acting as hydrogen-bonding donor [293, 294].

In our previous chapter, we utilized the self-assembly process for the generation of hierarchically ordered structures, to generate a polymer-based nanoparticle system. However, despite having a potential therapeutic effect in comparison to PTX, yet faces disadvantages associated with the conventional delivery system, such as drug efflux $[295,296]$ and nontargetability, due to the presence of extracellular matrix [297]. Thus, the goal of this chapter was to generate PTX nanoformulations by combining pharmaceutical excipients that not only impart solubilization of PTX but also contribute to chemosensitization, such as prevention of development of drug resistance. In our pilot study, the generation of a series of pharmaceutical small-molecule excipient-based PTX formulations, and then screening for specific/strong binding, lower particle size, and enhanced cellular uptake in BC cells, indicate that the TAbound PTX nanoparticles (TAP NPs) formulation is the best candidate for further study. Therefore, the aim of this study was to design, optimize, and evaluate the improved in vitro therapeutic efficacy of TAP NPs in BC.

\section{Materials and Methods}

\section{Chemicals and Solvents}

Aquaplex $^{\circledR}$ (randomly methylated $\beta$-cyclodextrin, catalogue no. AP-OLEC2), Trappsol® (carboxymethyl- $\beta$-cyclodextrin (CM- $\beta$-CD), catalogue no. TCMBPY), $\beta$-cyclodextrin ( $\beta$-CD, catalogue no. TBCD-P), and hydroxypropyl- $\beta$-cyclodextrin (HP- $\beta$-CD, catalogue no. THPB-P) were received from CTD Holdings, Inc. (Alachua, FL). L-ascorbic acid (L-AC, catalogue no. A7506), D- $\alpha$-tocopherol (D- $\alpha$-TP, catalogue no. 57668), Tween 80 (T80, catalogue no. P1754), D-mannitol (DM, catalogue no. M4125), D-sorbitol (DS, catalogue no. S1876), citric acid (CA, catalogue no. 251275), tannic acid (TA, catalogue no. 403040), and 4-(2-Hydroxyethyl) piperazine-1-ethanesulfonic acid, N-(2-Hydroxyethyl)piperazine-N'-(2-ethanesulfonic acid) (HEPES, catalogue no. H3375) were purchased from Aldrich (St. Louis, MO, USA). LC-MS/MS mobile phase solvents (methanol, formic acid and acetonitrile) were purchased from Aldrich. All of these chemicals, reagents, and solvents were analytical or HPLC grade and were used as received without further purification.

\section{Cell Culture}

The BC cell lines (MDA-MB-231 and MCF-7) were purchased from American Type Culture Collection (ATCC, Manassas, VA, USA). These cell lines were cultured in Dulbecco's Modified Eagle Medium (DMEM) that was supplemented with phenol red, 10\% fetal bovine 
serum, $100 \mathrm{U} / \mathrm{ml}$ penicillin and $100 \mathrm{U} / \mathrm{ml}$ streptomycin (Thermo Fisher Scientific, Grand Island, NY). For all experiments, cell lines were incubated at $37^{\circ} \mathrm{C}$ in a humidified $5 \% \mathrm{CO}_{2}$ and $95 \%$ air atmosphere (Thermo Fisher Scientific, Waltham, MA, USA). At 80-90\% confluency, these cells were trypsinized, counted using Trypan Blue $(0.4 \%)$ and seeded as per specific experiments. Cells were allowed to adhere to the plate surface overnight before implementing any further treatment.

\section{Preparation of Nanoparticles}

We have screened a set of eleven commonly used water-soluble pharmaceutical excipients/small molecules Aquaplex ${ }^{\circledR}$, Trappsol ${ }^{\circledR}, \mathrm{L}-\mathrm{AC}, \mathrm{CA}, \mathrm{DM}, \beta-\mathrm{CD}, \mathrm{HP}-\beta-\mathrm{CD}, \mathrm{D}-\alpha-\mathrm{TP}$, DS, T80, and TA to achieve the best PTX nanoformulation. The PTX nanoparticles were prepared by the solvent evaporation method. Being lipophilic, PTX was dissolved in acetone (stock concentration, $1 \mathrm{mg} / \mathrm{ml})$. Pharmaceutical excipient(s) $(5 \mathrm{mg})$ were dissolved in $1 \mathrm{ml}$ of ultrapure water (Milli-Q ${ }^{\circledR}$ Integral ultrapure water, $18.2 \mathrm{M} \Omega-\mathrm{cm}$ ) in $8 \mathrm{ml}$ flat-bottom glass vials on a Benchmark digital magnetic stir plate (400 rpm, ABC Scientific, Glendale, CA, USA) at room temperature. To this solution, PTX in acetone solution $(100 \mu \mathrm{lof} 1 \mathrm{mg} / \mathrm{ml})$ was slowly added dropwise to this solution. The stirring speed was increased to $900 \mathrm{rpm}$ and the solution was allowed to stir overnight to ensure that all of the acetone evaporated, leaving behind the nano self-assembly of small molecule and PTX, referred to as pharmaceutical excipient-PTX nanoparticles (PE-PTX NPs). For particle size, zeta potential, cellular uptake, and hemolysis experiments, a fresh batch of PE-PTX nanoparticles was always prepared. Note: the tannic acidPTX nanoformulation will be referred to as TAP NPs (Tannic Acid-Paclitaxel Nanoparticles). This is a specifically optimized formulation with low particle size, superior cellular internalization, and better hemocompatibility.

\section{Particle Size and Zeta Potential}

The hydrodynamic mean particle size (nm) and the size distribution/polydispersity (PDI) of PE-PTX NPs were measured by Zetasizer (Nano ZS, Malvern Instruments, Malvern, UK) based on the dynamic light scattering principle. These measurements were performed at $25^{\circ} \mathrm{C}$ with an angle of detection of $173^{\circ}$. For this determination, $50 \mu 1$ of $100 \mu \mathrm{g} / \mathrm{ml}$ of PE-PTX NPs were added to $1 \mathrm{ml}$ of ultrapure water and subjected to probe sonication (VirSonic Ultrasonic Cell Disrupter 100, The VirTis Company, Gardiner, NY) for 30 secs. The average diameter and distribution of particle sizes of the samples were reported from 3 runs (each run took $\sim 2 \mathrm{~min}$ ). The zeta potential of these nanoparticles ( $50 \mu \mathrm{l}$ of $100 \mu \mathrm{g} / \mathrm{ml}$ PE-PTX NPs in $1 \mathrm{ml}$ of 1 X PBS) was determined by a laser Doppler microelectrophoresis technique using Zetasizer. Each measurement was carried out in triplicate (each reading $=30$ runs). Additionally, we determined the particle size and zeta potential of the optimized formulation (TAP NPs) in HEPES buffer at $\mathrm{pH} 6,7,7.4$ and 8 to investigate the alteration in size distribution and charge with the change in $\mathrm{pH}$. For this purpose, freshly prepared $1 \mathrm{mM}$ of HEPES buffer using HEPES salt in ultrapure water was prepared. The $\mathrm{pH}$ of the buffer solutions was adjusted with $0.1 \mathrm{~N} \mathrm{HCl}$ and $\mathrm{NaOH}$ solutions and stored at $25^{\circ} \mathrm{C}$. 


\section{Quenching Assay}

Binding affinities of the pharmaceutical excipients to PTX (PTX conjugated to Oregon green 488 conjugate with a Flutax-2, Molecular Probes Inc., Life Technology, OR, USA) were determined following fluorescence decay of PTX using a SpectraMax Plus plate reader (Molecular Devices, Sunnyvale, CA). PTX conjugated to Oregon green 488 was used instead of PTX for this experiment. The PTX conjugated to Oregon green 488 at a concentration of $5 \mu \mathrm{g} / \mathrm{ml}$ was set for this study. The concentrations of PE solutions were as follows: $0,5,10,15,20,30$, 80 , and $130 \mu \mathrm{g} / \mathrm{ml}$. The fluorescence spectra were obtained by exciting these solutions at $496 \mathrm{~nm}$ and emission set between 450-600 $\mathrm{nm}$. All measurements were performed at room temperature. The fluorescence decay profiles of PE-PTX samples were used to calculate the number of binding sites and the binding constant $(\mathrm{k})$ [298].

\section{Cellular Uptake}

The cellular uptake of these nanoformulations in MDA-MB-231 and MCF-7 BC cells was determined by tracking fluorescent dye C6-loaded NPs. C6 was loaded in pharmaceutical excipient nanoparticles (PE-NPs) similarly as described in Section 2.3. For the qualitative cellular uptake experiment, $0.5 \times 10^{6}$ cells were seeded in a 12 -well plate and allowed to adhere to the plate surface overnight. On the next day, the cells were treated with PE-NPs at a concentration of $5 \mu \mathrm{M}$ for 3 hours. After treatment, cells were washed with 1X PBS, then phenol red-free DMEM medium was added and imaged under the GFP channel (Ex $470 \mathrm{~nm} / \mathrm{Em} 525$ $\mathrm{nm}$ ) of EVOS® 214 FL Imaging System (AMF4300, Life Technologies, Carlsbad, CA, USA). For semi-quantification of C6 loaded in PE-NPs inside the BC cells, they were trypsinized using $0.25 \%$ Trypsin-EDTA, pelleted down, washed twice with $1 \mathrm{X}$ PBS, collected, and dispersed in 1 $\mathrm{ml}$ phenol red-free DMEM medium. These cell suspensions were injected into the Accuri C6 Flow Cytometer (Accuri Cytometer, Inc., Ann Arbor, MI, USA) and fluorescence levels were detected in FL1 channel using 220 FITC/GFP green filter with an excitation at $488 \mathrm{~nm}$ (Blue laser, $530 \pm 15 \mathrm{~nm}$ ).

\section{Hemocompatibility}

The hemolysis assay was performed to determine the hemocompatibility of PE-PTX NPs with freshly collected human blood samples from Interstate Blood Bank, Inc., (IBBI, Memphis, TN, USA). The IBBI has appropriate ethical approval and authority to collect blood samples and distribute for research and clinical work. The blood sample $(1 \mathrm{ml})$ was centrifuged at $2000 \mathrm{rpm}$ for 5 mins, then the supernatant was discarded and the red blood cell (RBC) pellet was suspended in $10 \mathrm{ml}$ isotonic PBS and $200 \mu \mathrm{l}$ of cell suspension was used for the hemocompatibility assay. This RBC solution was treated with 10-100 $\mu \mathrm{g} /$ well PTX (in DMSO) or an equivalent concentration of PTX in PE-PTX NPs in a 96-well plate and incubated for 1 hour at $37^{\circ} \mathrm{C}$. For this experiment, PBS and sodium dodecyl sulfate (SDS at $1 \mathrm{mg} / \mathrm{ml}$ ) served as negative and positive experimental controls, respectively. After the incubation period, the hemoglobin released into the medium was collected and transferred into a fresh 96-well plate and hemoglobin optical density was quantified using a spectrophotometer (Cytation 3 imaging 
reader, BioTeK Winooski, VT, USA) at $\lambda_{\max } 570 \mathrm{~nm}$. The percentage of hemolysis was calculated using Equation 2-1. To confirm our results, a few drops of the treated RBCs were collected after the quantification and smeared on a glass slide using the coverslip and imaged using the $\operatorname{EVOS}^{\circledR}$ FL Imaging System (AMF4300, Life Technologies, Carlsbad, CA, USA).

\section{Physiochemical Characterization of TAP NP Formulation}

Freshly prepared solution samples of TAP NPs were lyophilized in the Labconco Freeze Dry System $\left(-52^{\circ} \mathrm{C} 133 \times 10^{-3} \mathrm{~m}\right.$ Bar; Labconco, Kansas City, MO, USA). The freeze-dried samples were used to record physicochemical characterization data, such as Fourier Transform Infrared (FTIR) spectra; thermogravimetric analyzer (TGA) scans; and X-ray diffraction (XRD data. FTIR spectra of TAP NPs were recorded on a Universal Attenuated Total Reflectance (UATR) Accessory plate by a Spectrum 100 FTIR spectrometer (Waltham, MA). The lyophilized samples were placed on the tip of the UATR diamond objective and a pressure of $90 \mathrm{~N} / \mathrm{cm}^{2}$ was applied. Spectra were acquired between 4000 and $650 \mathrm{~cm}^{-1}$ at a scanning speed of $4 \mathrm{~cm}^{-1}$ for 32 scans. The averaged data were presented as FTIR spectra $\left(\% \mathrm{~T} v s . \mathrm{cm}^{-1}\right)$ and analyzed using Spectrum 100 software. A Q50 thermogravimetric analyzer (TA Instruments, New Castle, DE) was used for TGA analysis under a dry nitrogen atmosphere with a flow rate and heating rate of $10 \mathrm{ml} / \mathrm{min}$ and $10^{\circ} \mathrm{C} / \mathrm{min}$, respectively. Thermogravimetric profiles were recorded from $50^{\circ} \mathrm{C}$ to $500^{\circ} \mathrm{C}$. A Rigaku D/Max-B diffractometer (Rigaku Americas Corp, Woodlands, TX) with cobalt-alpha radiation $(\mathrm{k}=1.5 \AA)$ was used for phase identification for crystalline material analysis. XRD patterns were acquired over $2 \theta$ range from $25^{\circ}-70^{\circ} \mathrm{C}$. The morphology and particle size of TAP NPs (after drying on grids) were determined using a JEOL 200EX transmission electron microscope (TEM) (JEOL Ltd, Tokyo, Japan) coupled with an AMT camera. For this study, TAP NPs $(20 \mu \mathrm{l}$ of $100 \mu \mathrm{g}$ PTX-equivalent/ml of TAP NPs $)$ were placed onto the shiny side of the copper grid (150 mesh, Electron Microscopy Sciences, PA, USA). A $2 \% \mathrm{w} / \mathrm{v}$ aqueous solution of uranyl acetate was used to stain TAP NPs to increase contrast property. The air-dried TAP NPs were imaged under the TEM at a direct magnification of $100,000 x$.

\section{Quantitative Analysis of TAP NPs}

\section{LC-MS/MS analysis}

The concentrations of PTX in the TAP NPs were measured by liquid chromatographytandem mass spectrometry (LC-MS/MS) using an LC system (Shimadzu Corporation, Kyoto, Japan) connected to a Triple Quad 5500 tandem mass spectrometer (AB SCIEX, Framingham, MA). The mobile phase was water with $0.1 \%$ formic acid and acetonitrile with $0.1 \%$ formic acid $(\mathrm{v} / \mathrm{v})$. The gradient elution was as follows: the starting condition was set at $50 \%$ mobile phase B and was kept at 50\% for the first min. The percentage mobile phase B was then increased from $50 \%$ to $100 \%$ from $1-2 \mathrm{~min}$, maintained at $100 \%$ up to $4 \mathrm{mins}$, and then decreased to $50 \%$ up to 5 mins, and maintained at 50\% until 6 mins elapsed time. The flow rate was set at $0.8 \mathrm{ml} / \mathrm{min}$ and total run time was 6 mins, with a retention time of 2.36 mins for PTX. Docetaxel (DTX) was used as an internal standard. Separation was achieved using XTerra ${ }^{\circledR}$ MS C18 Column 
Reversed-Phase $3.5 \mu \mathrm{m}$ Spherical Hybrid, $4.6 \mathrm{~mm}$ x $50 \mathrm{~mm}$ (\#186000432, Waters, Milford, MA). The following mass spectrometry ionization parameters were used: positive electrospray ionization mode, curtain gas pressure set at 20 psi, collision-activated dissociation at 10 psi, ion spray voltage at $5500 \mathrm{~V}$, the temperature at $60^{\circ} \mathrm{C}$, gas 1 and gas 2 pressure at 50.0 psi, declustering potential at $51.0 \mathrm{~V}$, entrance potential at $10.0 \mathrm{~V}$, collision energy at $21.0 \mathrm{~V}$, and collision exit potential at $24.0 \mathrm{~V}$. Analytes were quantified using multiple reaction monitoring to monitor the ion transitions of $\mathrm{m} / \mathrm{z} 876.3 \rightarrow 308.0$ for PTX and $\mathrm{m} / \mathrm{z}$ of $830.4 \rightarrow 549.2$ for DTX. The calibration standards for the analysis of drug encapsulation efficiency study were prepared in PBS, whereas CelLytic ${ }^{\mathrm{TM}}$ M (C2978) was used for the calibration standard preparation for cellular uptake study. The range of linear response of PTX was $0.039-40 \mu \mathrm{g} / \mathrm{ml}$ with $\mathrm{R}^{2}=0.9916$ in PBS and $0.01-10 \mu \mathrm{g} / \mathrm{ml}$ with $\mathrm{R}^{2}=0.9959$ in cell lytic buffer.

\section{Encapsulation efficiency}

Encapsulation or entrapment efficiency (EE) of PTX in TAP NPs was quantified by using LC-MS/MS. TAP NPs were centrifuged at 10,000 rpm for 10 mins to remove larger particles and non-encapsulated drug as a pellet. Supernatants were analyzed for drug content using LCMS/MS. The EE was calculated by Equation 3-1.

\section{$E E=[$ Amount of drug in TAP NPs $(\mu g) /$ Initial amount of drug $(\mu g)] \times 100$ (Eq. 3-1) \\ Intracellular drug uptake}

To determine in vitro intracellular concentration of PTX in MDA-MB-231 cells, cells were seeded in $150 \mathrm{~mm}$ petri dishes and allowed to attain $60-70 \%$ confluency. Each of the petri dishes at the defined time point was treated with $500 \mathrm{ng}$ of PTX or equivalent concentration of PTX in TAP NPs. Control cells without any treatment served as a control for this study. After 2, 4, and 6 hours of treatment, cells were washed with 1X PBS to remove any unwanted drug adhering to the cell surface, followed by trypsinization and centrifuging at $1000 \mathrm{rpm}$ for 5 mins. For extraction of internalized drug from cells, the trypsinized and centrifuged pellet was resuspended in CelLytic ${ }^{\mathrm{TM}} \mathrm{M}$ buffer and sonicated using 30 cycles of Ultrasonic Homogenizer (Biologics, Inc.), active every 10 secs for a 5 mins duration at $200 \mathrm{~W}$, on an ice bath. The internalized drug extracted from cells was measured by LC-MS/MS. Data were represented in triplicates or greater number of determinations.

\section{Biological Assays}

\section{Cell proliferation assay}

MTT assay was performed to assess the superior cytotoxicity of TAP NPs over PTX on BC cells. Briefly, $5 \times 10^{3}$ BC cells (MDA-MB-231 and MCF-7) in $100 \mu 1$ of media were seeded in a 96-well plate and allowed to rest in the incubator at $37^{\circ} \mathrm{C}$ overnight. Cells were treated with 1-50 nM PTX or 1-50 nM PTX-equivalent TAP NPs, and controls of untreated cells, DMSO, and plain TA, respectively, for 48 hours. Afterward, cells were incubated with $20 \mu 1$ of MTT reagent and incubated for 2-3 hours until the formazan salts formed. The media was aspirated out very 
carefully (as not to disturb any formazan crystals) and $100 \mu 1$ of DMSO was added to dissolve the salt formation [299]. The absorbance was recorded using a Microplate Reader (BioTeK Cytation 3, Winooski, VT, USA) at $570 \mathrm{~nm}$. Each treatment concentration was studied in triplicate determinations. The cell proliferation ability of PTX or TAP NPs was determined as the percentage in contrast to the control (DMSO or TA). Changes in cell morphology following treatment were imaged using EVOS $®$ FL Imaging System at 20X magnification.

\section{Clonogenic assay}

This study was performed to investigate the influence of TAP NPs on the clonogenic forming ability of BC cells. Briefly, MDA-MB-231 and MCF-7 (250 cells/well in $1 \mathrm{ml})$ were seeded in a 12-well plate and allowed to adhere to the plate and grow smaller colonies in 2 days. Then cells were treated with 0.25, 0.5, and $1 \mathrm{nM}$ PTX and 0.25, 0.5, and $1 \mathrm{nM} \mathrm{PTX-equivalent}$ TAP NPs and respective controls (no treatment and TA-treated BC cells). These cells were allowed to rest in the incubator for the next 15 days, with intermittent changing of medium but no drug added. After 15 days visible colonies were processed for staining with hematoxylin and imaged according to our previous reports [300]. The number of colonies formed was represented as bar graphs to represent the difference in the effect of each treatment group. The results are presented as the percentage of colonies in contrast to the control groups (non-treated cells).

\section{Cellular migration}

Cell migration was analyzed using the Boyden's chamber cell migration assay [301, 302]. Briefly, MDA-MB-231 $\left(0.5 \times 10^{5}\right)$ cells were suspended in serum-free media with 5 and $10 \mathrm{nM}$ of PTX or equivalent amounts in TAP NPs, added to the upper chamber of the 96-transwell inserts (catalog no.3374, Corning, NY, USA). These inserts were then placed inside the 96-well plates in pure media (10\% FBS). After 24 hours incubation, the migrated cells at the lower inside of the Transwell inserts were fixed with $4 \%$ formaldehyde and stained with crystal violet, with cells on the upper side of the insert being removed with a cotton swab. Inserts were imaged using the EVOS $^{\circledR}$ FL Imaging System, the number of migrated cells were counted and expressed as a percentage with respect to the migrated control cells (cells with no treatment).

\section{Cellular invasion}

Cellular invasion was measured using the BD Biocoat Matrigel Invasion Chambers (BD Biosciences) [302]. MDA-MB-231 $\left(3.5 \times 10^{4}\right.$ cells $)$ were seeded in the matrigel invasion chambers (BD Biosciences, Bedford, MA, USA) in serum-free medium and treated with 5 and $10 \mathrm{nM}$ PTX or 5 and $10 \mathrm{nM}$ equivalent PTX of TAP NPs for 24 hours. 10\% FBS medium was

placed into the lower chamber as chemoattractant. The invaded cells were fixed with methanol and stained using crystal violet. The plates were imaged and were counted as described in section 2.10.3. 


\section{Drug Resistance Assays}

\section{P-gp ATPase activity}

To investigate the effect of TAP NPs on the inhibition of P-gp efflux pump, the P-gpGlo $^{\mathrm{TM}}$ Assay Systems (Promega, USA) was used per manufacturer's instructions. This assay can be used to determine the effect of a compound to characterize inhibitors of P-gp ATPase activity. $\mathrm{Na}_{3} \mathrm{VO}_{4}$ is used as a negative control and verapamil is used as a positive control for drug stimulation of P-gp ATPase activity. The test compounds were PTX, TAP NPs, and TA, while untreated samples served as our experimental control. The manufacturer's protocol was followed for the experiment.

\section{Rhodamine-123 efflux assay}

To study the difference in P-gp expression with the treatment of TAP NPs, the RH123 accumulation assay was employed [303]. For this study, $0.5 \times 10^{6}$ of MDA-MB-231 BC cells were seeded in a 6-well plate and allowed to adhere to the surface overnight. The next day, cells were treated with 5 and $10 \mathrm{nM}$ of PTX or 5 and $10 \mathrm{nM}$ of PTX-equivalent TAP NPs. After 48 hours, $2.62 \mu \mathrm{mol} / 1$ of RH123 was incubated with cells for 30 mins. Following incubation, cells were washed with 1X PBS and imaged using phenol red-free DMEM medium under an EVOS ${ }^{\circledR}$ 214 FL Imaging System [260]. For semi-quantification, cells were then trypsinized, pelleted down, washed with 1X PBS, and suspended in phenol red-free DMEM medium and analyzed using Accuri C6 Flow Cytometer in FL1 channel with an excitation at $488 \mathrm{~nm}$ (Blue laser, $530 \pm$ $15 \mathrm{~nm})$.

\section{$\beta$-tubulin Stabilization}

The treatment effect of TAP NPs on tubulin stabilization was studied by confocal imaging. For this, MDA-MB-231 $\left(0.25 \times 10^{6}\right)$ cells were grown on coverslips and were treated with 5 and $10 \mathrm{nM}$ PTX or PTX equivalent TAP NPs for 8 hours. Blank TA and cells with DMSO treatment served as our experimental control. The treated cells were fixed in $4 \%$ paraformaldehyde in PBS, pH 7.4 for 15 mins at room temperature. Fixation was subsequently quenched with $0.1 \mathrm{M}$ glycine and washed in PBS. After washing, cells were blocked in 10\% goat serum $/ 5 \%$ BSA $/ 0.5 \%$ TritonX-100 in PBS for 1 hour at RT. Following blocking, sections were incubated with 1:50 Anti-beta Tubulin antibody (\#ab6046, Abcam, Cambridge, MA) overnight at $4^{\circ} \mathrm{C}$, on a rocker at 20 oscillations/min. The next day, after washing in blocking buffer three times for 5 mins each, cells were probed using Goat anti-Rabbit IgG $(\mathrm{H}+\mathrm{L})$ Cross-Adsorbed Secondary Antibody, Alexa Fluor 488 (catalog no. A11008) and DAPI to label nuclei for 1 hour. After washing in PBS four times for 5 mins each, coverslips were mounted using Prolong Diamond Antifade Mountant (catalog no. P36961) and imaged using a laser confocal microscope (Carl Zeiss LSM 710, Thornwood, NY, USA) with a $40 \times 0.7$ NA oil immersion objective. All images were captured using the same laser power and gain settings to ensure comparability for intensity analysis. Fluorescent intensities were quantified using Image J. Data were represented by measuring fluorescent intensities from 18 individual cells. 


\section{Western Blotting}

MDA-MB-231 BC cells $\left(1 \times 10^{6}\right)$ were seeded in a $100 \mathrm{~mm}$ dish and allowed to adhere to the surface overnight. The next day, these plates were treated with $10 \mathrm{nM}$ PTX or $10 \mathrm{nM}$ PTXequivalent TAP NPs, and their respective controls of untreated cells and TA treated cells, for 48 hours. Cell lysates were formed using 2X SDS lysis buffer (Santa Cruz Biotechnology, Santa Cruz, CA) and the protein was quantified using Bradford assay and run on a SDS-PAGE [304, 305]. The expressions of specific proteins were determined with an immunoblotting assay as described above. Protein expressions confirmed by immunoblotting were $\beta$-actin (\#4967), BclxL (\#2762), Bad (\#9268), p53 (\#2527), Cleaved Caspase-7 (\#9491), MDR1/ABCB1 (\#13342), and Cleaved PARP (\#9548) (Cell Signaling Technologies, Danvers, MA). The protein bands were developed under the ChemiDoc ${ }^{\mathrm{TM}}$ MP System (Bio-Rad, Hercules, CA, USA) with the help of Lumi-Light Detection Kit.

\section{RNA Extraction and Microarray Studies}

The gene regulatory effects of TAP NPs on BC cells were evaluated by microarray studies. For this, MDA-MB-231 cells $\left(1 \times 10^{6}\right)$ were seeded in a $100 \mathrm{~mm}$ dish and allowed to reach $60-70 \%$ confluency before treating with PTX and TAP NPs for 24 hours. The RNA was extracted, purified, and processed as described in our previous study [306]. The RNA integrity was confirmed through NanoDrop ${ }^{\text {TM }} 2000$ (Thermo Fisher Scientific, Waltham, MA, USA). In this study, the clariom ${ }^{\mathrm{TM}} \mathrm{S}$ Human gene array (Affymetrix, Santa Clara, CA, USA) was employed as gene chips, and the assay was performed per the manufacturer's protocol.

\section{Statistical Analysis}

All data were generated and analyzed using GraphPad Prism 5.03 Software (GraphPad Software, San Diego, CA) and results are presented as mean \pm standard error of mean (SEM). Statistical significance was performed using a student's t-test analysis when comparing between two groups. When comparing the statistical significance among multiple treatment groups oneway ANOVA post-hoc Tukey HSD test was performed and a $p$-value $<0.05$, was considered significant when compared to control.

\section{Results}

\section{Screening of Pharmaceutical Excipients for PTX Delivery}

The main purpose of conducting this study was to develop a nanoformulation against BC that would possess superior anticancer activity [307]. For this purpose, we developed nanoformulation(s) of PTX utilizing eleven commonly used pharmaceutical excipients: Aquaplex $^{\circledR}$, L-ascorbic acid, citric acid, beta-cyclodextrin, carboxymethyl-beta-cyclodextrin, Dmannitol, hydroxypropyl-beta-cyclodextrin, D- $\alpha$-tocopherol, D-sorbitol, tannic acid, and Tween 
80. The drug-loaded, self-complexed nanoparticles were prepared with a self-assembly technique. Incorporation of PTX dissolved in acetone occurred when the drug solution is placed in a polymer excipient solution overnight. Evaporation of acetone allows nanoparticles to form a suspension in an aqueous phase. The resultant nanoparticles generated with all the above pharmaceutical excipients were screened and characterized for particle size and size distribution, surface charge, drug binding efficiency, hemocompatibility, and cellular uptake in cancer cells (Table 3-1). Figure 3-1A represents particle sizes of all the PE-PTX NPs generated. Among these, TAP NPs and Tween 80-PTX NPs demonstrate good particle sizes of $\leq 100 \mathrm{~nm}$, which is considered optimal from a drug delivery standpoint. However, Tween 80-PTX NPs, despite having a low particle size of $80.68 \pm 7.13$, the lower number of available binding sites and low binding rates limits its further optimization.

The PTX-conjugated Oregon green 488 quenching ability with all pharmaceutical excipients was determined with a fluorescence spectrophotometer. The fluorescence intensity of the PTX dye decreases with the increase of pharmaceutical excipients. This quenching is at a maximum with tannic acid as seen in Table 3-1 (Column 4: Dye quenching). In contrast to all the other ten pharmaceutical excipients employed, TA has the minimum area under the curve (AUC), demonstrating that PTX dye is quenched by TA, which causes the most reduction in AUC (Figure 3-1B). This is directly correlated to the binding ability of tannic acid to PTX by attractive forces such as hydrogen bonding and electrostatic interactions. The superior binding efficiency with tannic acid was confirmed from the higher number of binding sites (1.4) and binding constant (0.03) over other pharmaceutical excipients. Table 3-1 shows ascorbic acid has a similar reduction in AUC, the total number of binding sites and binding constant as tannic acid; however, its large particle size of $321.63 \mathrm{~nm}$ (as formulated into nanoparticles) limits its further use.

Tannic acid-PTX nanoparticles (TAP NPs) formation by the self-assembly process was confirmed by space-filling energy-minimized (MM2) molecular modeling using ChemBio3D Ultra 14 Suite (CambridgeSoft Corporation, A subsidiary of PerkinElmer, Inc.). The spacefilling energy-minimized molecular models of different ratios of TA:PTX (1:1, 2:1, 3:1, 4:1, 5:1, $6: 1,8: 1,10: 1$ and 12:1) indicates thermodynamically feasible TA-PTX self-assembly formation (Figure 3-2A). Among many ratios employed for the formation of TAP NPs, TA: PTX ratio of 24:1 leads to a well-organized nanoparticle structure with particle size and zeta potential of $102.22 \pm 14.05 \mathrm{~nm}$ and $-8.85 \pm 0.44 \mathrm{mV}$. The presence of excess TA was utilized as a stabilizing agent of the nanoformulation, as previously reported [140]. The illustration of the self-assembly formation of TAP NPs is shown in Figure 3-2B. The nanoparticles generated should be well internalized inside the cytosolic membrane to be able to deliver therapeutic benefits. In this regard, we loaded the nanoparticles with a fluorescent dye (C6) to be able to detect through the GFP channel of the microscope/flow cytometer. This study was investigated using BC cell lines MDA-MB-231 and MCF7. The cellular internalization was observed to be in the order of tannic acid $>$ Tween $80>$ CM- $\beta$-CD $>$ ascorbic acid $>$ aquaplex $>\beta-C D>$ HP- $\beta-C D>$ D-mannitol $>$ citric acid $>$ tocopherol $>$ D-sorbitol for MDA-MB-231, and tannic acid $>$ Tween $80>$ CM- $\beta$-CD $>$ ascorbic acid $>$ aquaplex $>$ citric acid $>\beta-\mathrm{CD}>$ tocopherol $>$ D-mannitol $>$ HP- $\beta-\mathrm{CD}>\mathrm{D}-$ sorbitol for MCF7 (Table 3-1, Column 7 and 8). Tannic acid demonstrated significantly increased cellular uptake in contrast to all the C6-loaded excipient nanoparticles, through 
Table 3-1. Preliminary evaluation of pharmaceutical excipient-based paclitaxel nanoparticles for developing $\mathrm{BC}$ therapeutics

\begin{tabular}{|c|c|c|c|c|c|c|c|c|}
\hline \multirow[b]{2}{*}{$\begin{array}{l}\text { Pharmaceutical } \\
\text { excipient }^{\mathrm{a}}\end{array}$} & \multirow[b]{2}{*}{$\begin{array}{l}\text { Particle } \\
\text { size } \\
(\mathrm{nm})\end{array}$} & \multirow[b]{2}{*}{$\begin{array}{l}\text { Zeta } \\
\text { potential } \\
(\mathrm{mV})\end{array}$} & \multicolumn{3}{|c|}{$\begin{array}{c}\text { Fluorescence } \\
\text { quenching }\end{array}$} & \multicolumn{2}{|c|}{$\begin{array}{c}\text { Mean } \\
\text { fluorescence } \\
\text { intensity } \\
\left(\mathrm{MFI} \times \mathbf{1 0}^{\mathbf{5}}\right)^{\mathrm{c}} \\
\end{array}$} & \multirow[b]{2}{*}{$\begin{array}{l}\% \\
\text { hemolysis } \\
\text { at } 50 \mu \mathrm{g}^{\mathrm{d}}\end{array}$} \\
\hline & & & $\begin{array}{l}\text { Dye } \\
\text { quenching } \\
\left(\text { AUC } \times 10^{6}\right)^{b}\end{array}$ & $\begin{array}{l}\text { Number } \\
\text { of } \\
\text { binding } \\
\text { site(s) }\end{array}$ & $\begin{array}{l}\text { Binding } \\
\text { rate } \\
\text { constant } \\
(\mathbf{k}) \\
\end{array}$ & $\begin{array}{l}\text { MDA- } \\
\text { MB- } \\
231\end{array}$ & MCF7 & \\
\hline Aquaplex $^{\circledR}$ & $\begin{array}{l}355.93 \pm \\
15.97\end{array}$ & $\begin{array}{l}-4.32 \pm \\
0.81\end{array}$ & 1.65 & 0.80 & 0.003 & $\begin{array}{l}1.37 \pm \\
0.092\end{array}$ & $\begin{array}{l}0.86+ \\
0.014\end{array}$ & $\begin{array}{l}2.06 \pm \\
0.01\end{array}$ \\
\hline $\begin{array}{l}\text { L-Ascorbic acid } \\
\text { (L-AC) }\end{array}$ & $\begin{array}{c}321.63 \pm \\
23.22\end{array}$ & $\begin{array}{l}-2.25 \pm \\
0.65\end{array}$ & 0.94 & 1.38 & 0.035 & $\begin{array}{l}1.87 \pm \\
0.031\end{array}$ & $\begin{array}{l}0.98 \pm \\
0.010\end{array}$ & $\begin{array}{l}3.78 \pm \\
0.34\end{array}$ \\
\hline Citric acid (CA) & $\begin{array}{l}323.60 \pm \\
27.90\end{array}$ & $\begin{array}{l}-3.60 \pm \\
0.65\end{array}$ & 1.17 & 0.57 & 0.008 & $\begin{array}{l}0.23 \pm \\
0.002\end{array}$ & $\begin{array}{l}0.40 \pm \\
0.002\end{array}$ & $\begin{array}{l}50.17 \pm \\
0.35\end{array}$ \\
\hline $\begin{array}{l}\text { Trappsol }{ }^{\circledR}, \\
\text { (CarboxyMethyl- } \\
\beta-C D)\end{array}$ & $\begin{array}{l}252.50 \pm \\
14.51\end{array}$ & $\begin{array}{l}-6.98 \pm \\
0.78\end{array}$ & 1.26 & 0.73 & 0.012 & $\begin{array}{l}2.18 \pm \\
0.045\end{array}$ & $\begin{array}{l}2.18 \pm \\
0.067\end{array}$ & $\begin{array}{l}7.21 \pm \\
0.59\end{array}$ \\
\hline $\begin{array}{l}\beta \text {-cyclodextrin } \\
(\beta-C D)\end{array}$ & $\begin{array}{l}254.31 \pm \\
7.58\end{array}$ & $\begin{array}{l}-5.07 \pm \\
0.66\end{array}$ & 1.34 & 1.05 & 0.012 & $\begin{array}{l}0.49+ \\
0.008\end{array}$ & $\begin{array}{l}0.32+ \\
0.004\end{array}$ & $\begin{array}{l}12.71 \pm \\
0.68\end{array}$ \\
\hline $\begin{array}{l}\text { Hydroxypropyl } \\
\beta \text {-cyclodextrin } \\
\text { (HP- } \beta \text {-CD) }\end{array}$ & $\begin{array}{l}213.56+ \\
8.55\end{array}$ & $\begin{array}{l}-5.59 \pm \\
0.46\end{array}$ & 1.47 & 0.94 & 0.010 & $\begin{array}{l}0.47 \pm \\
0.004\end{array}$ & $\begin{array}{l}0.18+ \\
0.001\end{array}$ & $\begin{array}{l}19.24 \pm \\
3.82\end{array}$ \\
\hline $\begin{array}{l}\text { D-Mannitol } \\
\text { (DM) }\end{array}$ & $\begin{array}{l}308.33 \pm \\
19.77\end{array}$ & $\begin{array}{l}-8.87 \pm \\
0.46\end{array}$ & 1.50 & 0.41 & 0.001 & $\begin{array}{l}0.27 \pm \\
0.001\end{array}$ & $\begin{array}{l}0.23 \pm \\
0.013\end{array}$ & $\begin{array}{l}1.03 \pm \\
0.03\end{array}$ \\
\hline D-Sorbitol (DS) & $\begin{array}{l}149.76 \pm \\
4.61\end{array}$ & $\begin{array}{l}-7.05 \pm \\
0.30\end{array}$ & 2.58 & 0.63 & 0.001 & $\begin{array}{l}0.14+ \\
0.026\end{array}$ & $\begin{array}{l}0.14 \pm \\
0.017\end{array}$ & $\begin{array}{l}6.18 \pm \\
0.59\end{array}$ \\
\hline Tannic acid (TA) & $\begin{array}{l}102.22 \pm \\
14.05\end{array}$ & $\begin{array}{l}-8.85 \pm \\
0.44\end{array}$ & 0.94 & 1.40 & 0.038 & $\begin{array}{l}4.24 \pm \\
0.045\end{array}$ & $\begin{array}{l}4.91 \pm \\
0.030\end{array}$ & $\begin{array}{l}5.15 \pm \\
0.02\end{array}$ \\
\hline $\begin{array}{l}\text { D- } \alpha \text {-tocopherol } \\
(D-\alpha-T P)\end{array}$ & $\begin{array}{l}161.06 \pm \\
21.25\end{array}$ & $\begin{array}{l}-6.28 \pm \\
0.05\end{array}$ & 1.70 & 0.46 & 0.003 & $\begin{array}{l}0.18 \pm \\
0.001\end{array}$ & $\begin{array}{l}0.21 \pm \\
0.004\end{array}$ & $\begin{array}{l}9.96 \pm \\
0.34\end{array}$ \\
\hline Tween 80 (T80) & $\begin{array}{l}80.68 \pm \\
7.13\end{array}$ & $\begin{array}{l}-6.63 \pm \\
0.39\end{array}$ & 2.20 & 0.43 & 0.001 & $\begin{array}{l}2.30 \pm \\
0.266\end{array}$ & $\begin{array}{l}2.49 \pm \\
0.149\end{array}$ & $\begin{array}{l}9.96 \pm \\
0.02\end{array}$ \\
\hline
\end{tabular}

a $5 \mathrm{mg} / \mathrm{ml}$ of each pharmaceutical excipient was used to generate PE-PTX nanoparticles

${ }^{\mathrm{b}}$ Oregon green 488 conjugated to PTX $(5 \mu \mathrm{g} / \mathrm{ml})$ was quenched with $130 \mu \mathrm{g} / \mathrm{ml}$ of PE solutions

${ }^{\mathrm{c}}$ Coumarin 6 loaded Pharmaceutical excipient nanoparticles $(5 \mu \mathrm{g} / \mathrm{ml}$ equivalent coumarin 6 nanoparticles) were treated on breast cancer cells

${ }^{d}$ ASTM E2524-08 standard considers hemolysis of $\leq 5 \%$ safe compounds are safe for human consumption. 


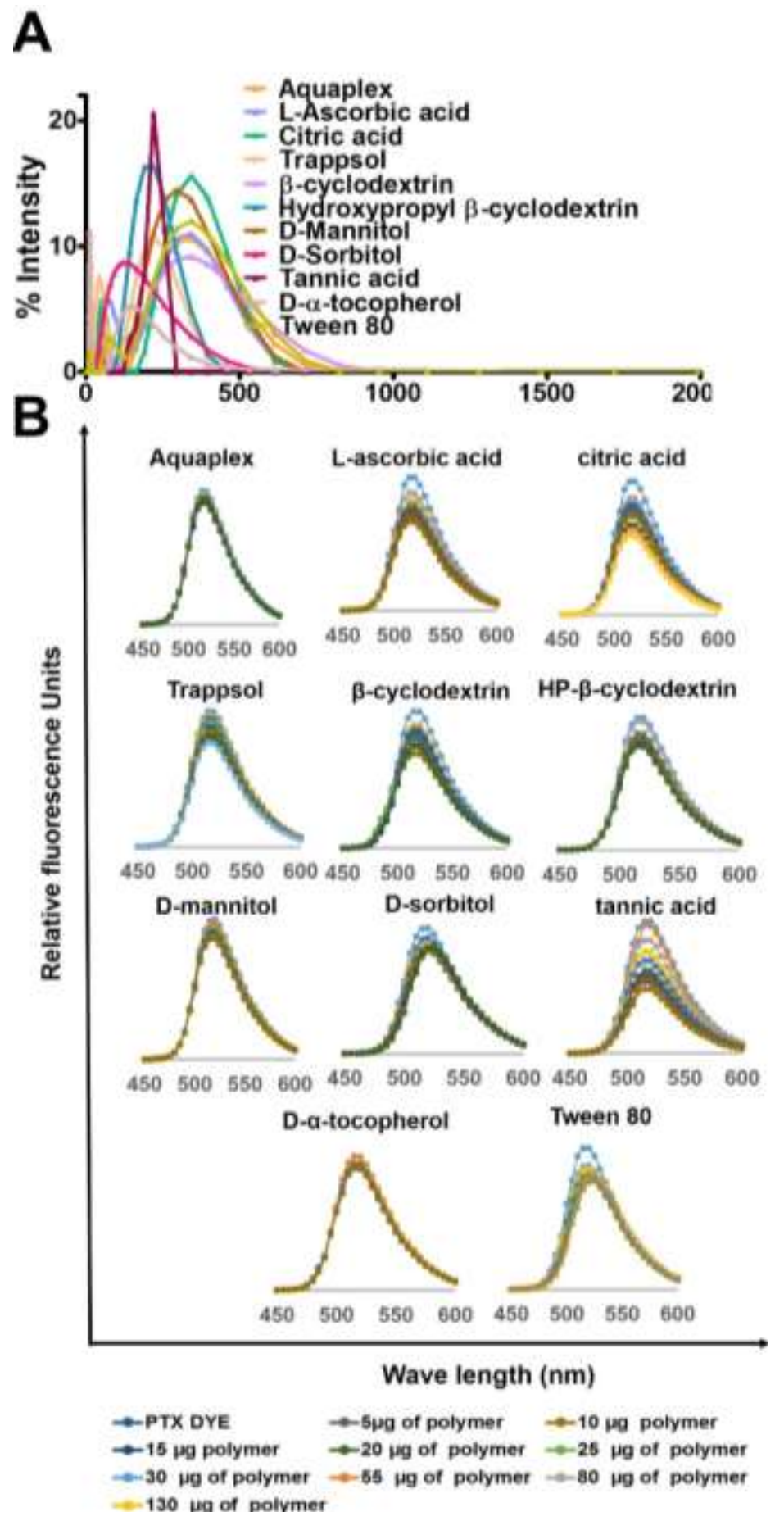

Figure 3-1. Characterization during the screening process

A) Particle size measurement of all the eleven developed nanoformulation as measured by DLS.

B) The binding potential was confirmed by quenching of PTX conjugated dye (PTX, Oregon

Green 488 conjugate (Flutax-2, Molecular Probes Inc., Life Technology, OR, USA)) with pharmaceutical excipient-PTX (PE-PTX) NPs (5-130 $\mu \mathrm{g} / \mathrm{ml})$. 


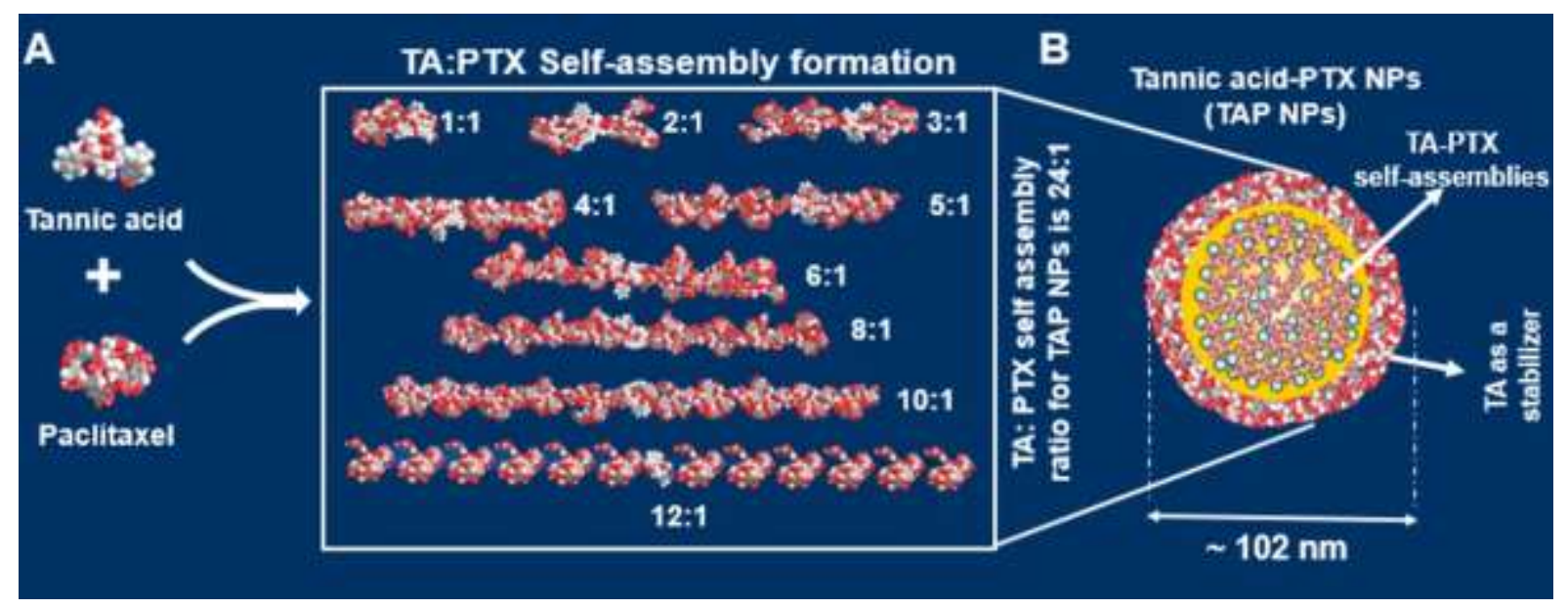

Figure 3-2. Formation and optimization of TAP NPs

A) Space-filling energy-minimized (MM2) molecular modeling shows generation of thermodynamically stable TA-PTX self-assembly. Various ratios as shown above were employed to generate MM2 models of TA-PTX self-assembly. B) Hypothetical representation of 24:1 ratio of TA: PTX self- assembly, so called Tannic acid Nanoparticles (TAP NPs). For visual clarity, PTX in hypothetical structure is represented as 
determinations both by flow cytometry (Table 3-1) and the EVOS ${ }^{\circledR}$ FL Imaging System (Figure 3-3A). To implement our generated nanoparticles from bench to bedside, the toxicity profile of the formulations needs to be assessed. Hemocompatibility assay of these pharmaceutical excipient-PTX nanoparticles (PE-PTX NPs) were investigated at varying concentrations ranging from 10-100 $\mu \mathrm{g} /$ well PTX alone and 10-100 $\mu \mathrm{g} /$ well PTX-equivalent PE-PTX NPs. Table 3-1 data show that $50 \mu \mathrm{g}$ PTX is toxic to RBCs with $\sim 63 \%$ hemolysis (Figure 3-3B). There was no significant difference observed with varying concentrations of TAP NPs, while many PE-PTX NPs were not toxic in nature. Indeed, TAP NPs exhibited a safer profile. This behavior was further evaluated by the morphological changes of red blood cells that are incurred after incubating with PTX alone and PE-PTX NPs, respectively (Figure 3-3C). TAP NPs demonstrated no morphological changes in the structure of RBCs on treatment (similar to negative control or PBS).

Altogether, the screening of various pharmaceutical excipient based PTX nanoparticles, studied by particle size, zeta potential, PTX binding, cellular uptake, and hemolysis data suggests that tannic acid is considered a safe vehicle for PTX delivery, and thus is considered to be the best candidate for further investigation.

\section{Characterization of TAP NPs}

DLS data demonstrated that TAP NPs measured approximately $102.22 \pm 14.05 \mathrm{~nm}$ (Figure 3-4A). No specific change in particle size was seen for 6 days after storing the nanoformulation at room temperature, except for day 7 (Inset of Figure 3-4A), which suggests that TAP NPs are stable at room temperature for at least a week. Although, extended stability study needs to be validated in future studies. The effect of changes in $\mathrm{pH}$ with nanoparticle generation was examined by using HEPES buffer ( $\mathrm{pH}$ range 6-8). Change in size is represented in Figure 3-4B. These data further suggested that at all recorded $\mathrm{pH}$ values $(6,7,7.4,8)$, TAP NPs demonstrate well-assembled nanoparticles of $154.13,116,82.16,71.55 \mathrm{~nm}$ at $\mathrm{pH}$ values 6 , 7, 7.4 and 8 respectively. TA is a weakly acidic polyphenol with gallic acid molecules that are capable of donating protons. At higher $\mathrm{pH}$, more phenolate binding sites are available for effective complexation with PTX. This leads to more packed self-assembly formation of TAP NPs with lower particle size at higher $\mathrm{pH} 8$ compared to $\mathrm{pH} 6$ (Fig. 3-4B). The TAP nanoparticles exhibited spherical morphology as seen in Figure 3-4C. As seen under the TEM, TAP NPs measure an average of $60.55 \pm 3.03 \mathrm{~nm}$.

To confirm the presence of PTX and tannic acid in TAP NPs, the molecular composition was investigated by FTIR spectroscopy (Figure 3-4D). The characteristic peaks at $1700 \mathrm{~cm}^{-1}$, $1603 \mathrm{~cm}^{-1}$, and $1198 \mathrm{~cm}^{-1}$ belong to $\mathrm{C}=\mathrm{O}$ stretching, aromatic $\mathrm{C}=\mathrm{C}$ stretching, and phenolic $\mathrm{C}-\mathrm{O}$ stretching for tannic acid, whereas characteristic vibrational peaks of PTX include $3350 \mathrm{~cm}^{-1}(\mathrm{~N}$ $\mathrm{H}$ stretching) and $1703 \mathrm{~cm}^{-1}(\mathrm{C}=\mathrm{O}$ stretching) $[24,25]$. The characteristic peak of phenolic $\mathrm{C}-\mathrm{O}$ at $1198 \mathrm{~cm}^{-1}$ of TA is shifted to $1100 \mathrm{~cm}^{-1}$ in TAP NPs, while the characteristic band of the aromatic ring at $1603 \mathrm{~cm}^{-1}$ and ester band at $1700 \mathrm{~cm}^{-1}$ are observed in TAP NPs. Also, the main characteristic peaks of PTX at $3350 \mathrm{~cm}^{-1}$ and $1650 \mathrm{~cm}^{-1}$ can be detected in TAP NPs due to N-H and $\mathrm{C}-\mathrm{C}$ stretching, respectively, indicating the presence of PTX in TAP NPs. Interactions 


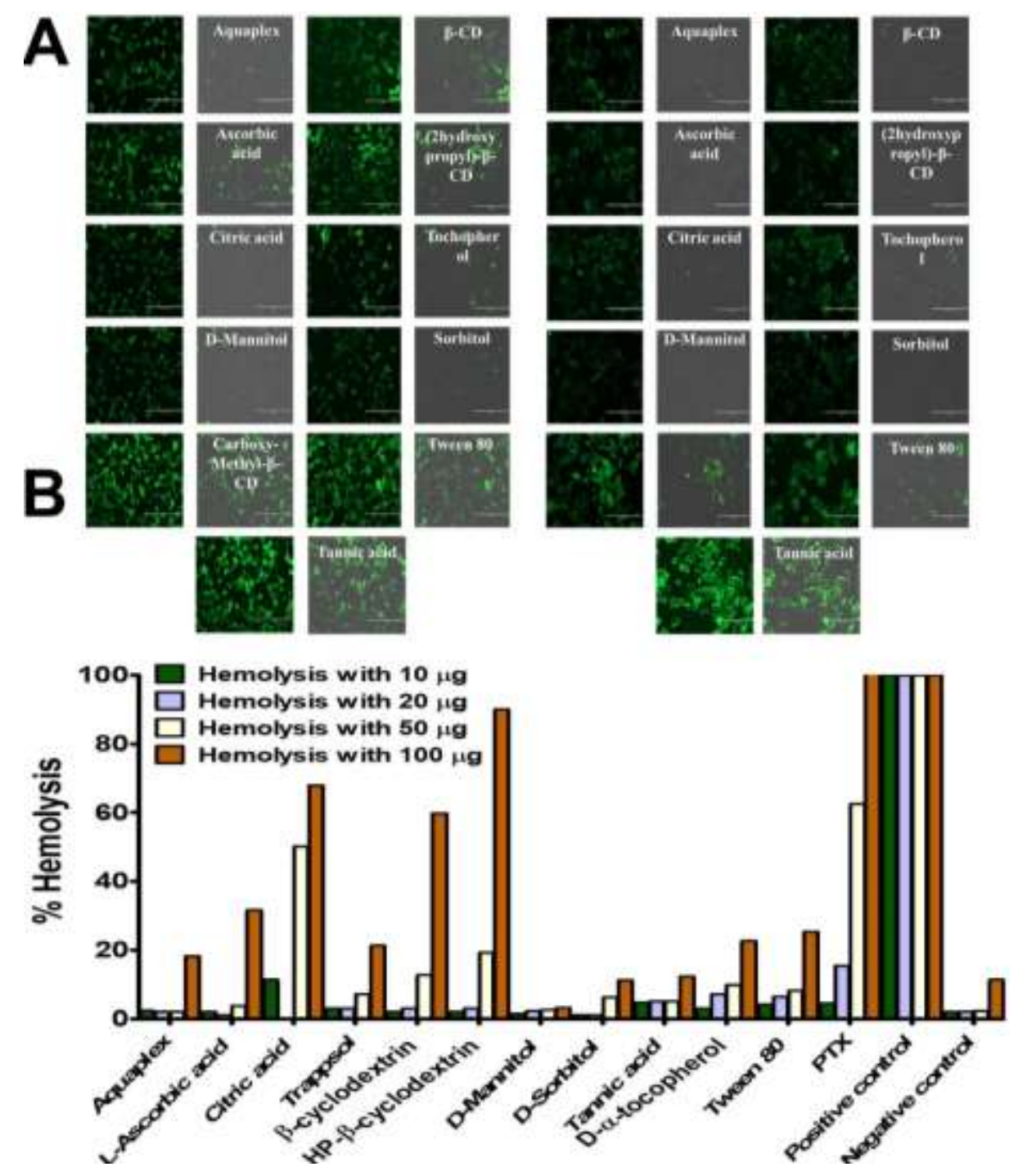

Figure 3-3. In vitro screening during screening process

A) Qualitative internalization efficiency of PE-NPs in MDA-MB-231 cells for 3 hours. Images were captured after incubation with EVOS ${ }^{\circ}$ FL Imaging System (AMF4300, Life Technologies, Carlsbad, CA, USA). B) Hemocompatibility of PE-PTX NPs was assessed by incubating 10-100 $\mu \mathrm{g} / \mathrm{well}$ PTX equivalent of nanoparticles in each well for 1 hour. (SDS, $1 \mathrm{mg} / \mathrm{ml}$ ) served as a positive control while PBS was used as a negative control. No significant difference in percentage of hemolysis was observed for TAP NPs at all tested concentrations. C) Qualitative assessment of hemocompatibility of PE-PTX NPs by incubating nanoparticles with RBC for 1 hour. Images demonstrate intact membrane morphology for negative control (PBS), whereas positive control (SDS) and most of the other PE-PTX NPs exhibited toxicity (seen from the disintegrating morphology of RBC). 


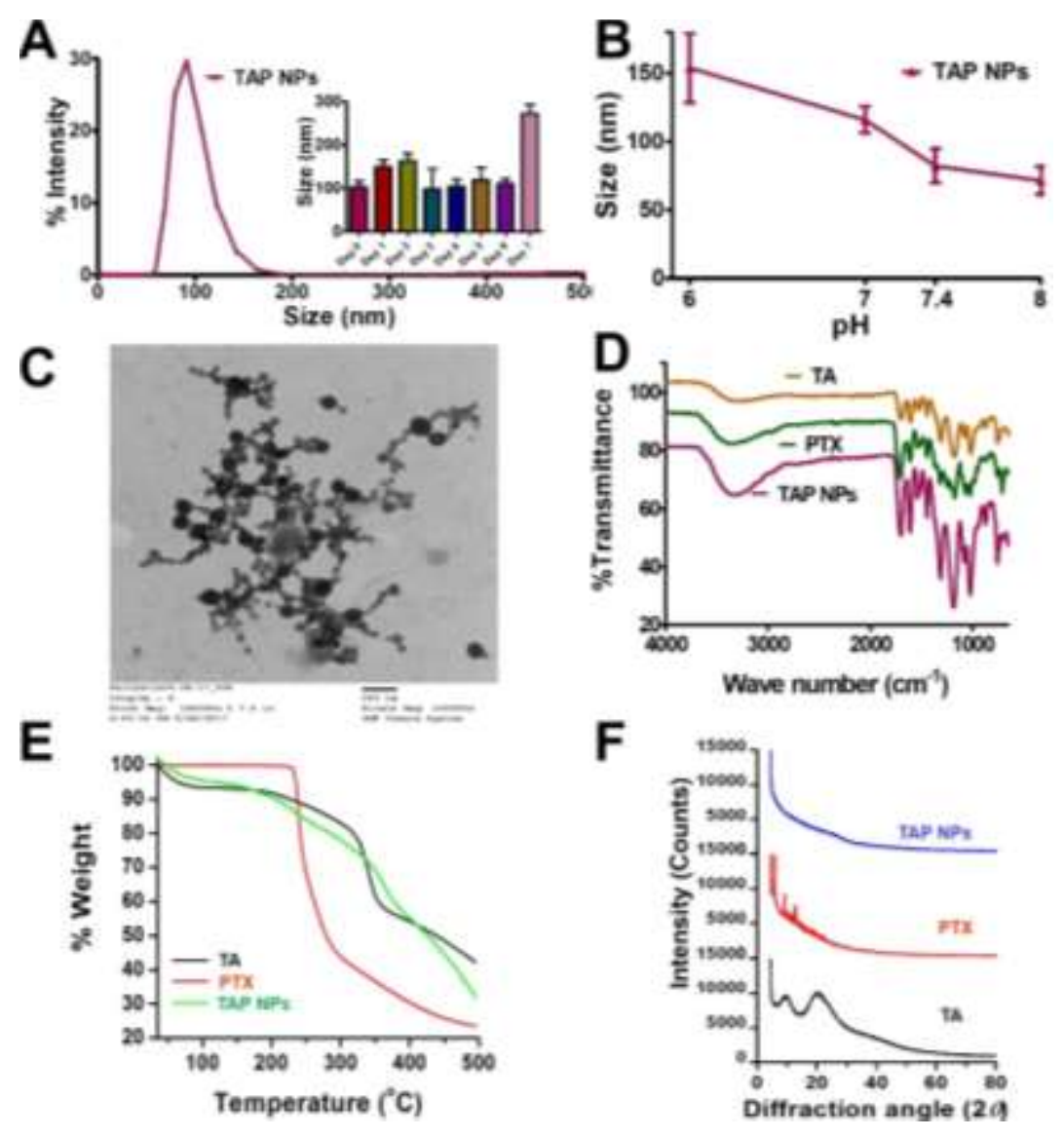

Figure 3-4. Physio-chemical characterization of TAP NPs

A-B) Dynamic light scattering analysis of TAP NPs: stability over 1-week and in different $\mathrm{pH}$ solutions as measured using Zetasizer (Nano ZS, Malvern Instruments, Malvern, UK) at $25^{\circ} \mathrm{C}$. A) TAP NPs exhibited particle size of $102.22 \pm 14.05 \mathrm{~nm}$. Inset: representative particle size of TAP NPs stored at $25^{\circ} \mathrm{C}$ for 1-week. B) Stability of TAP NPs in HEPES buffer solutions (pH 6, 7, 7.4 and 8). Data presented as mean + standard error of the mean $(\mathrm{n}=3)$. C) A representative transmission electron microscopic (TEM) image of TAP NPs. Image was acquired by using JEOL 200EX TEM at a direct magnification of $100,000 \times($ scale $=100 \mathrm{~nm})$. D) Fourier-transform Infrared (FTIR) spectral analysis of TA, PTX and TAP NPs acquired on a Universal Attenuated Total Reflectance (UATR) accessory plate by a Spectrum 100 FTIR spectrophotometer (Waltham, MA), between 4000 and $650 \mathrm{~cm}^{-1}$ at a scanning speed of $4 \mathrm{~cm}^{-1}$ for 32 scans. TAP NPs show characteristic TA peaks at 1603 and $1700 \mathrm{~cm}^{-1}$ and PTX peaks at 3350 and $1650 \mathrm{~cm}^{-1}$, confirming the presence of functional moieties of TA and PTX in TAP NPs. E)

Thermogravimetric analysis (TGA) was recorded for TA, PTX and TAP NPS from 50 to $500^{\circ} \mathrm{C}$ by a Rigaku D/Max-B diffractometer (Rigaku Americas Corp, Woodlands, TX) with cobaltalpha radiation $(\mathrm{k}=1.5 \AA)$. There is no significant change in thermogram of TAP NPs in comparison to TA and PTX. F) X-ray diffraction (XRD) was acquired at $2 \theta$ range of $25-70^{\circ} \mathrm{C}$ suggesting TAP NPs has both TA and PTX present in amorphous or dissolution state. 
between tannic acid and PTX are mainly through the ester functions, aromatic rings, and hydrogen bonding mediated by the phenolic hydroxyl groups, which suggest attractive forces occur between tannic acid and PTX in TAP NPs, but there is no evidence of covalent bond formation. The physical state of tannic acid and PTX in TAP NPs may influence various drug delivery properties. Thermogravimetric and XRD analyses were employed to study such interactions and the physical state of TA and PTX complex structure. Thermograms of tannic acid (Figure 3-4E) show two major weight loss peaks between $55-100^{\circ} \mathrm{C}$ due to curing, reformation, and oxidation, while another appears at $250-400^{\circ} \mathrm{C}$ due to severe degradation of tannin intermolecular bonding. In the case of PTX, it exhibited a strong degradation pattern above $228^{\circ} \mathrm{C}$. When PTX is incorporated into TA assemblies, TAP NPs demonstrate degradation patterns very similar to tannic acid, but it retains its PTX degradation phenomenon too. XRD patterns of PTX demonstrate several sharp peaks around 5.38, 9.62, 10.74, 11.85, and $12.96^{\circ}$ (20) (Figure 3-4F), while TA shows broad peaks at 9.75 and $20.63^{\circ}(2 \theta)$. However, XRD of TAP NPs does not show such characteristic peaks of either TA or PTX. This suggests that both TA and PTX are present in the form of amorphous or dissolved states in TAP NPs.

\section{Encapsulation Efficiency and Intracellular Drug Uptake of TAP NPs}

The aims of drug entrapment are either enhanced delivery or improved uptake in cancer cells, and reduction in off-target toxicity. The encapsulation efficiency of PTX in TAP NPs was determined by LCMS/MS and was $>96.49 \pm 0.43 \%$. Drug activity is dependent on crossing the cellular membrane and forming drug depots in cellular compartments. For this reason, qualitative analysis of cellular transport of C6 loaded in pharmaceutical excipient nanoparticles (PE-NPs) was examined in MDA-MB-231 cells in a time-dependent manner and cellular internalization of C6 was imaged by EVOS ${ }^{\circledR}$ FL Imaging System at 40X magnification, as seen in Figure 3-5B. After confirming the uptake of TAP NPs in BC cells, we examined cellular transport of PTX from a plain PTX drug solution vs. TAP NPs in BC cell line MDA-MB-231 in a time-dependent manner. Cellular internalization of the drug in cancer cells was analyzed using LC-MS/MS; see chromatogram: standard curve for the LC-MS/MS analysis (inset) (Figure 3-5A). The cellular internalization behavior of free PTX and TAP NPs are shown in Figure 3-5B. The maximum PTX concentration measured was $955.24+110.13 \mathrm{ng} / \mathrm{ml}$ for 6 hours from TAP NPs in contrast to free PTX, which attained a maximum concentration of $572+33.44 \mathrm{ng} / \mathrm{ml}$ after 6 hours of treatment. As seen in Figure 3-5B, it is evident that the plain PTX concentration was similar at all of the tested time points, suggesting PTX concentration inside BC cells was probably due to efflux by the P-gp transporters on the cell membrane. The drug concentration inside the cells increased over time with TAP NPs treatment, suggesting that significantly higher accumulation of drug was achieved (equivalent to $95.52 \%$ of drug internationalization through TAP NPs in 6 hours, whereas free PTX could only achieve a maximum of up to $57.19 \%$ in the same amount of time). A plausible mechanism for an enriched amount of PTX in the cellular compartment with TAP NPs is due to the presence of TA, which is reported to inhibit P-gp function by partly inhibiting ATPase [308]. We, therefore, suggest that TAP NPs provide higher drug concentration at the target and result in improved therapeutic efficacy over that of the free drug. 


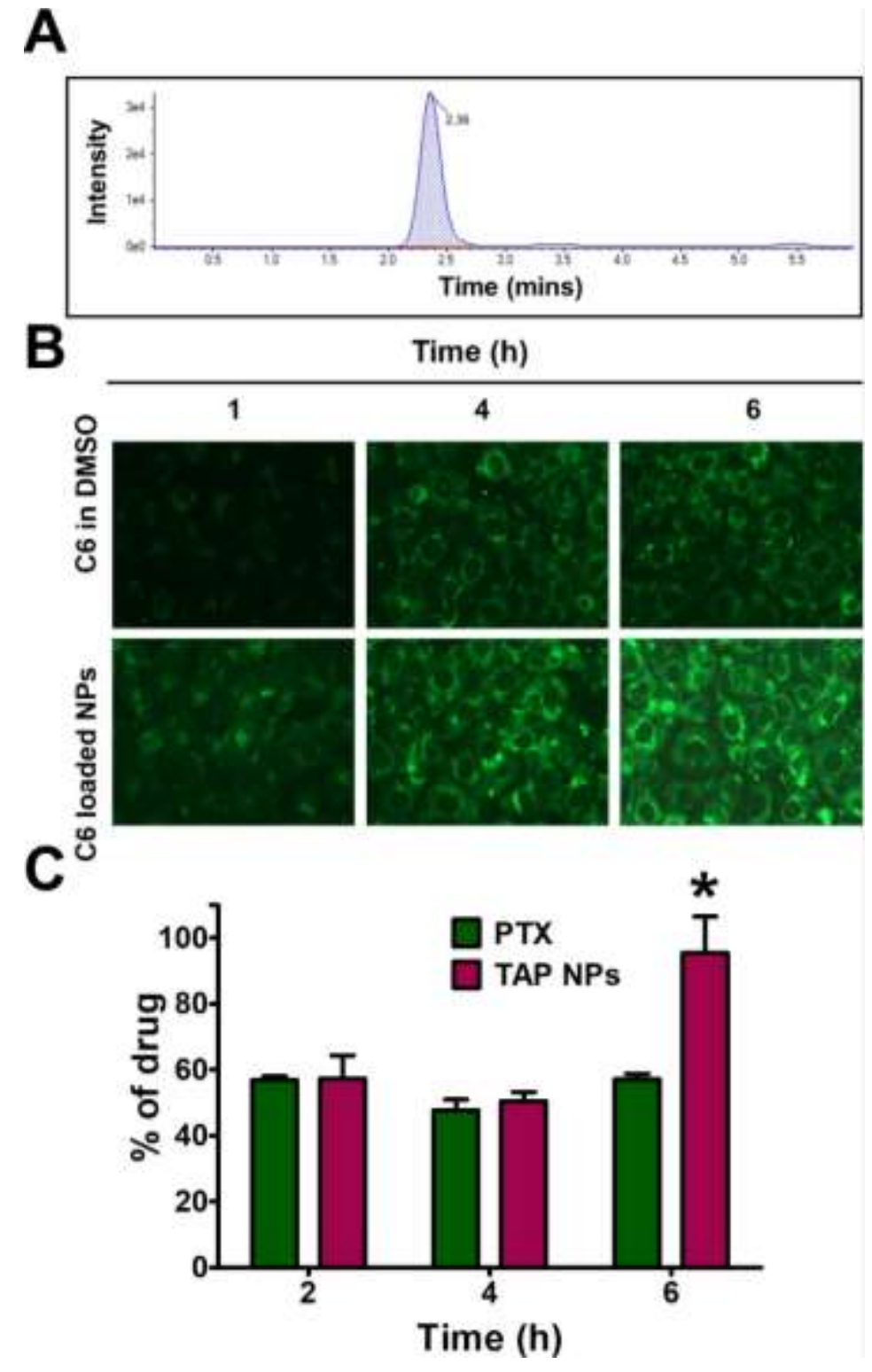

Figure 3-5. Intracellular uptake of TAP NPs in BC MDA-MB-231 cells A) Chromatogram of PTX from TAP NPs as seen by LC-MS/MS. B) Cellular uptake of C6 uptake in BC MDA-MB-231 cells were confirmed by fluorescence microscopy an EVOS® 214 FL Imaging System, in a time dependent manner with dye loaded nanoparticles. C) Intracellular uptake of TAP NPs by LC-MS/MS in MDA-MB-231 after treatment with PTX and TAP NPs for 2, 4 and 6 hours. Drug internalization of $95.52 \%$ was achieved by TAP NPs in 6 hours in contrast to only $57.19 \%$ by native drug PTX for the same time. Data presented as mean \pm standard error of the mean $(\mathrm{n}=3)$. A Liquid chromatography-tandem mass spectrometry (LCMS/MS) (Shimadzu Corporation, Kyoto, Japan) connected to Triple Quad 5500 tandem mass spectrometer (AB SCIEX, Framingham, MA. all tested concentrations. To study the migratory and invasive nature of cancer cells and the effect of TAP NPs and plain PTX, we conducted the Boyden migration assay and the Matrigel invasion assay on MDA-MB-231. 


\section{TAP NPs Inhibits Proliferation, Clonogenic Formation, Migration and Invasion in BC Cells}

The MTT assay was used to evaluate in vitro cytotoxicity effects of TAP NPs and PTX (50-1.5 nM equivalent) on MDA-MB-231 and MCF7 BC cells after 48 hours of treatment. Untreated cells served as a positive control for the experiment. As shown in Figure 3-6A, both TAP NPs and PTX exhibited a dose-dependent antiproliferative effect. After 48 hours of treatment, there was a significant reduction in $\mathrm{IC}_{50}$ values of TAP NPs compared to the native drug PTX. The $\mathrm{IC}_{50}$ values were $6.29 \mathrm{nM}$ and $14.34 \mathrm{nM}$ for TAP NPs in MDA-MB-231 and MCF7 respectively. A significant reduction in fold change of 2.62 and 1.94 in MDA-MB-231 and MCF7 was observed, respectively. This suggests TAP NPs are more potent in inhibiting the proliferation of BC cells than free PTX. Figure 3-6B shows a clear change in morphology of MDA-MB-231 and MCF7 on administration of TAP NPs and PTX (5 nM). To further confirm the superior cell proliferation effect of TAP NPs, the clonogenic potential of MDA-MB-231 and MCF7 were determined for two weeks after treatment with TAP NPs and PTX (Figure 3-6C through D).

Untreated cells and free PTX served as positive and negative control. As seen in Figure 3-7A,B, there was a significant inhibition of clonogenicity with TAP NPs in contrast to PTX at. Cells were treated with TAP NPs and free PTX (5 and $10 \mathrm{nM}$, PTX equivalent) and allowed to migrate across the Transwell inserts for 24 hours as shown in Figure 3-7A,B. No obvious inhibition was seen in control (untreated cells) or blank TA treatment, whereas TAP NPs showed significant migratory inhibition over free PTX at all tested concentrations, with 1.26- and 2.96fold inhibition at 5 and $10 \mathrm{nM}$, respectively. Similar results were achieved by the Matrigel invasion assay Figure 3-7C,D, where MDA-MB-231 cells were treated with 5 and $10 \mathrm{nM}$ of TAP NPs and PTX (PTX equivalent) for 24 hours. TAP NPs had similar inhibitory effects on invasive BC cells with $46.78 \%$ and $16.27 \%$ inhibition of cells in contrast to $64.62 \%$ and $49.53 \%$ inhibition observed with free PTX treatment at 5 and $10 \mathrm{nM}$, respectively. There was a statistical difference in the inhibition profile of TAP NPs at 5 and $10 \mathrm{~nm}$ in comparison to the native PTX at the same concentration. These results suggest that TAP NPs not only deliver enhanced therapeutic benefit over the free drug, but also inhibit cell migration and invasion, or possibly limit metastasis-related death for clinical applications.

\section{TAP NPs Lower P-gp Expression in BC Cells}

To evaluate P-gp function in cancer cells following treatment with TAP NPs and PTX, Pgp-Glo $^{\mathrm{TM}}$ assay and RH123 efflux assay were performed. The P-gp-Glo assay relies on the ATP dependence of the light-generating reaction of firefly luciferase. For this experiment, $\mathrm{Na}_{3} \mathrm{VO}_{4}$ is used as a selective inhibitor of P-gp and Verapamil, which is a substrate for P-gp transport, and may inhibit P-gp activity by competitively interfering with the transport of other substrates [309]. Since $\mathrm{Na}_{3} \mathrm{VO}_{4}$ inhibits P-gp activity, less ATP is consumed, resulting in brighter signals or higher average light units (RLU). TA has been previously reported [308, 310] to be involved in the modulation of drug efflux and shows similar P-gp inhibitory activity. However, higher consumption of ATP was observed, indicating PTX is being effluxed by the P-gp membrane, in 

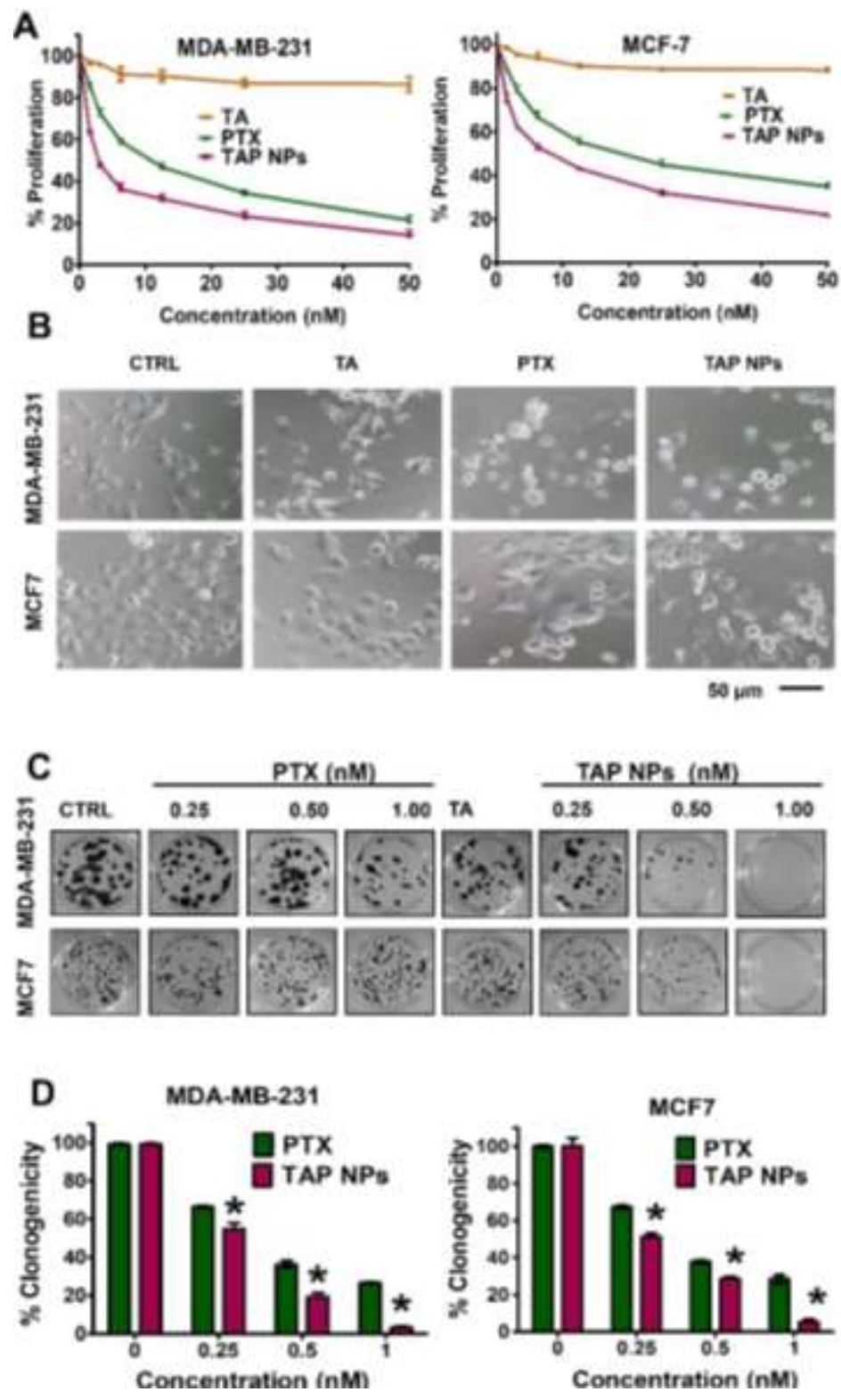

Figure 3-6. Anticancer efficacy of TAP NPs in BC cells

A) Cell proliferation by MTT assay markedly decreased proliferation of BC cells (MDA-MB231 and MCF7) after treatment with 1-50 nM PTX or TAP NPs for 48 hours. Untreated cells were used as control. Absorbance was recorded by a Microplate Reader (BioTeK Cytation 3, Winooski, VT, USA) at $570 \mathrm{~nm}$. B) Bright field microscopy images after MTT analysis show significant changes in cell morphology after 48 hours treatment with TAP NPs and PTX. Imaging was done by an $\operatorname{EVOS}^{\circledR}$ FL Imaging System (20X magnification, scale $\left.=200 \mu \mathrm{m}\right)$. C) Inhibition of colony formation by TAP NPs and PTX (equivalent to $0.25,0.5$ and $1 \mathrm{nM} \mathrm{PTX)} \mathrm{on}$ MDA-MB-231 and MCF7 cells for 15 days were observed for the colony forming ability of BC cells. Imaged in Multimage ${ }^{\mathrm{TM}}$ light cabinet. Data presented as mean \pm standard error of the mean $(\mathrm{n}=3) . \mathrm{D})$ 

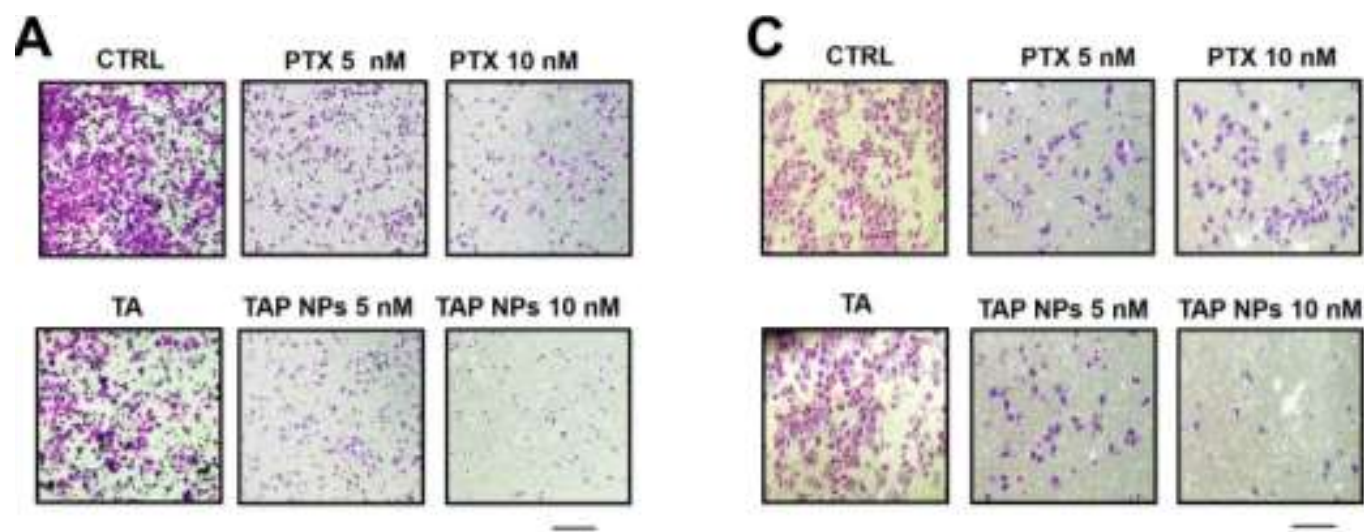

TAP NPS $5 \mathrm{nM}$ TAP NPs $10 \mathrm{nM}$

B
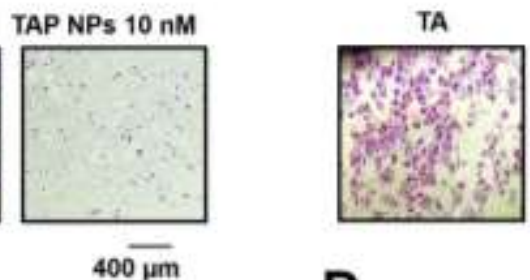

D
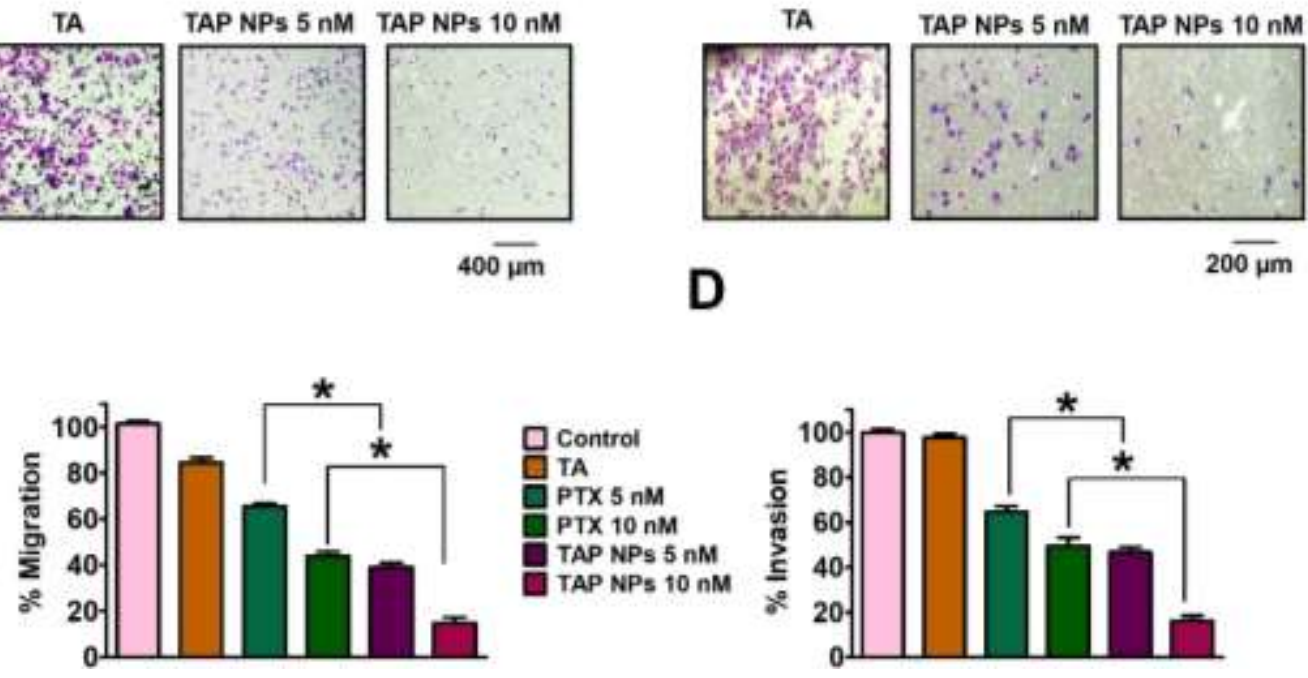

Figure 3-7. Anti-metastatic potential of TAP NPs

A) Migration assay to determine inhibition potential of BC (MDA-MB-231) cells after exposure to 5 and $10 \mathrm{nM}$ of PTX or TAP NPs (PTX equivalent) for 24 hours. Imaged using EVOS® FL Imaging System. B) Representative bar graphs of the number of migratory cells with each treatment group, where untreated (control) cells served as an experimental control and was considered as $100 \%$ and other treatment groups expressed as percentage with respect to the migrated control cells. B) Matrigel Invasion assay showing similar effects, upon treatment with TAP NPs and PTX (5 and $10 \mathrm{nM}$ equivalent to PTX) for 24 hours. C) Invasive cells following incubation with PTX and TAP NPs were fixed with methanol and stained using crystal violet. Imaged using EVOS ${ }^{\circledR}$ FL Imaging System. D) Percentage inhibition of invasive BC cells upon treatment and data represented as a bar graph with respect to untreated (control) cells which also served as our experimental control. Data represented as mean + standard error of the mean $(n=$ $3)$. 
contrast to TAP NPs, which are probably effluxed less due to the presence of TA in the formulation (Figure 3-8A). A statistical difference in the RLU of TA and TAP NPs in comparison to Verampil was observed, confirming the P-gp inhibitory effects of TA and because of TA in TAP NPs, as well. RH123 showed enhanced accumulation of the fluorescent dye, as shown in Figures 3-8B,C. RH123 is an established substrate for evaluating the ability to inhibit P-gp mediated transport. As seen in Figure 3-8C, statistically lower accumulation occurs with free PTX treatment, probably due to high P-gp expression, which extrudes drug/dye from the cells, in comparison to TAP NPs. As predicted, a higher fluorescence was observed with TAP NPs suggesting significant inhibition of drug efflux. This is correlated to the fact that tannic acid is known to have a chemosensitizing effect [310] on cancer cells that possess P-gp inhibitory efflux, which explains the enhanced accumulation achieved by TAP NPs. Overall, we can suggest that our TAP NPs developed for this study not only have the potential of providing enhanced therapeutic effects but also prevent the development of drug resistance by preventing or lowering drug efflux.

\section{Effect of TAP NPs on Microtubule Stabilization, Oncogenic Protein Expression, and Microarray Studies}

The molecular effects which PTX or the taxane group of drugs exhibit cause the microtubules to stabilize, promoting mitotic arrest and cell death [311]. To ensure that the molecular activity of microtubule stabilization is intact in TAP NPs and to compare the apoptotic ability against PTX, tubulin stabilization assay was conducted by immunostaining and imaging via confocal microscopy. As shown in Figure 3-9A, control cells show distinct microtubule bundles, which can undergo regular cellular processes such as cell division. In agreement with previous reports $[312,313]$, cells treated with TAP NPs and free PTX show distinct thicker microtubule bundles (more distinct at $10 \mathrm{nM}$ treatment). Graphical representation of the changes in fluorescent intensity was quantified using ImageJ software, and significant enhanced Mean Intensity (arbitrary units) was observed for both 5 and $10 \mathrm{nM}$ of TAP NPs in contrast to PTX (Figure 3-9B).

There was a statistically significant increase in microtubule bundles in TAP $10 \mathrm{nM}$ in comparison to PTX, $10 \mathrm{nM}$. Thus, suggesting greater cytotoxic effects that TAP NPs impart on $\mathrm{BC}$ cells. This is probably occurring because the majority of the native drug PTX is effluxed out by cancer cells, as demonstrated by the P-gp evaluation assays. This suggests that the tubulin stabilization process can inhibit mitosis, motility, and intracellular transport, eventually resulting in apoptotic cell death [311]. Further, to evaluate the effect of TAP NPs on the expression of proapoptotic, apoptotic, and pro-survival proteins, immunoblot analysis was conducted. As shown in Figure 3-9C, the decreased expression of Bcl-xL was observed with TAP NPs treatment, whereas enhanced expression was observed for pro-apoptotic and apoptosis-associated proteins (Bad, Cleaved PARP, and Cleaved Caspase-7). Furthermore, the decreased expression of multidrug resistance (MDR) protein (no resistance developed) and increased expression of p53 (tumor suppressor protein) confirms the activation of apoptosis by TAP NPs, which induce cancerous cell death. Microarray results suggest that TAP NPs promote pro-apoptotic 

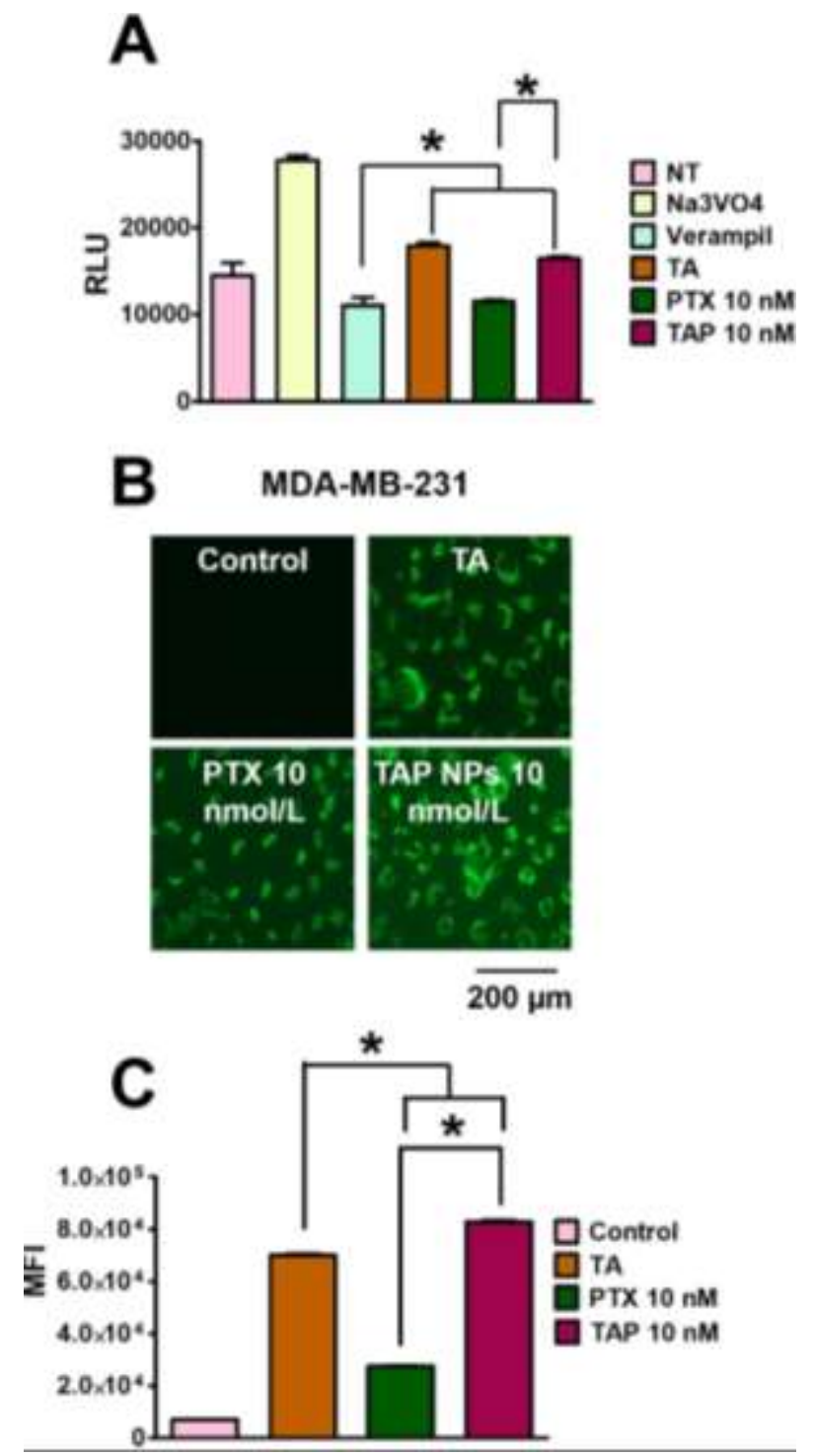

Figure 3-8. Inhibition of drug resistance by TAP NPs

A) P-gp-Glo assay showing ATP consumption by different treatment groups having variation in average light units (RLU). Na3VO4 and Verapamil were negative and positive controls respectively, where higher ATP consumption signifies lower RLU. TAP NPs show 1.42-fold higher RLU in contrast to native drug PTX, which is more effluxed out by the P-gp membranes. B) RH123 $(2.62 \mu \mathrm{M})$ dye shows higher accumulation in MDA-MB-231 cells after incubation for 30 mins when cells were treated for 48 hours with TAP NPs in contrast to PTX. TAP NPs exhibited significantly increased accumulation, suggesting TAP NPs prevent drug efflux. C) Mean fluorescence intensity in FL1 channel (488 excitation, Blue laser, $530+15 \mathrm{~nm}$, FITC/GFP) was measured for different treatment groups. PTX $10 \mathrm{nM}$ treatment showed 3-fold lower intensity over TAP NPs (equivalent to $10 \mathrm{nM}$ PTX). Data presented as mean + standard error of the mean $(n=3)$. 

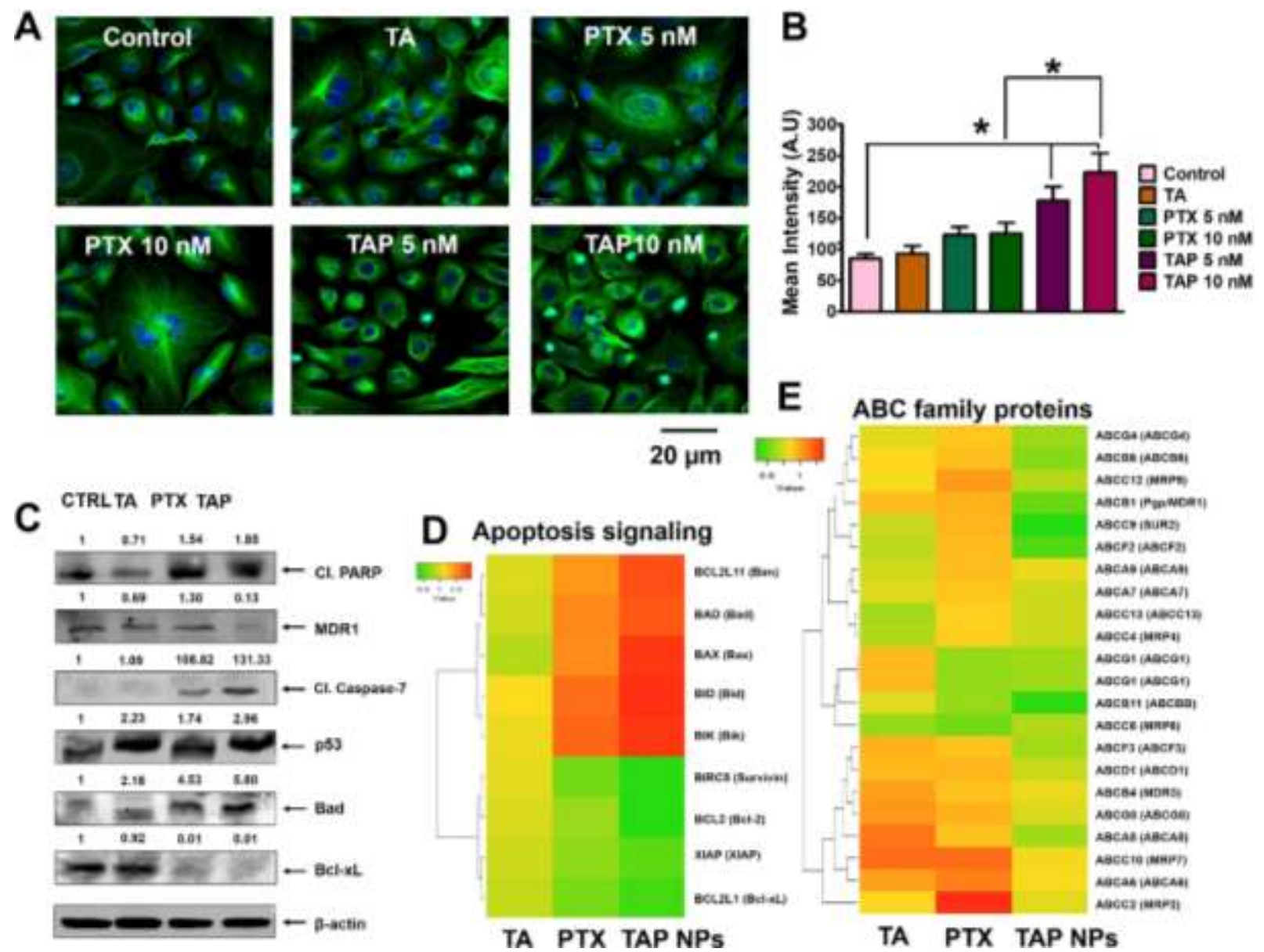

Figure 3-9. Molecular mechanism of TAP NPs in inducing apoptosis in BC cells

A) $\beta$-tubulin stabilization assay representing confocal images after treatment with 5 and $10 \mathrm{nM}$ PTX or PTX equivalent TAP NPs for 8 hours on MDA-MB-231. Distinct bundles of microtubules were seen in control cells suggesting their proliferating nature, which changed to thicker clusters of microtubules after treatment with TAP NPs and PTX, suggesting stabilization of microtubules accounting for the innate nature of the taxane drug. B) Quantitative analysis of the florescent intensity by Image J software shows significant lower Mean Intensity (arbitrary units $)$ in contrast to TAP NPs. Data represented as mean + standard error of the mean $(n=3)$. C) Western blot analysis of whole cell lysates of MDA-MB-231 that was treated with 10 nM PTX or $10 \mathrm{nM}$ PTX equivalent TAP NPs and their respective controls for 48 hours and immunoblotted for Cleaved PARP, MDR1/ABCB1, Cleaved Caspase-7, p53, Bad, Bcl-xL and $\beta$ actin. The results were consistent in two independent sets of experiments. D) Heat map of differentially regulated gene expression of pro-apoptotic and anti-apoptotic signaling leading to apoptosis in MDA-MB-231 BC cells after exposure to TAP NPs. E) Heat map representing gene expression of $\mathrm{ABC}$ family. 
and inhibit anti-apoptotic gene expression validated through a heat map (Figure 3-9D). Furthermore, the gene level expression of ABC family, depicting inhibition of MDR/P-gp was confirmed through microarray analysis, as represented in a heat map (Figure 3-9E).

\section{Discussion}

PTX is a well-known potent anticancer drug used in the treatment of various cancers including BC. Still, there is a constant effort made towards achieving better therapeutic benefits of PTX antitumor effects in cancer. Furthermore, there is a paucity of information regarding the PTX mechanisms of action which need to be better understood. Literature evidence demonstrates nanoparticle technology plays a crucial role in the field of medicine. Currently, more than 65 nanomedicine-based formulations are FDA-approved and enjoy widespread use in clinical applications. In chemotherapy, nanoformulations are often used to 1) minimize toxicity associated with anticancer agents, 2) enhance pharmacokinetics, pharmacodynamics, and the bioavailability of drug molecules and 3) preferentially target, accumulate, and release drugs at the tumor site, due to the Enhanced Permeation and Retention (EPR) mechanism. Among many nanomedicines, Abraxane ${ }^{\circledR}$ is a frequently used chemotherapy in clinical practice, with about 1 billion-unit sales. This implies that PTX is still a widely used anticancer molecule in current. treatment regimens. Therefore, it is apparent that a simple nanoformulation made up of a pharmaceutical excipient that has chemosensitization properties would improve therapeutic outcomes and could greatly impact the current treatment modality. The choice of polymer excipient dictates the physicochemical and biological properties of drug nanoformulation. Toward this end, we report in this study the preparation of PTX nanoparticles using eleven structurally different polymer excipients. Our aim is to avoid complex chemistry and to not use organic solvents, to prevent nonspecific uptake. Self-assembly formation (through favorable particle size generation and zeta potential), efficient excipient-drug complexation (shown by fluorescence dye quenching and evaluating binding efficiency), favorable hemolysis behavior (hemoglobin release from RBC due to toxicity), and cellular uptake by cancer cells (shown by appropriate analytical methods) were successfully utilized to synthesize and choose the best formulation that could be used for a future nanomedicine for PTX drug delivery. Our screening data, from a set of eleven commonly used pharmaceutical excipients for generating PTX nanoparticles, confirmed that tannic acid is highly recommended to achieve an instantaneous self-assembly formation through hydrogen bonding and other attractive forces (Table 3-1, and Figures 3-1 through 3-3).

Pharmaceutical excipients are often used to improve the aqueous solubility of various hydrophobic drugs, which can be applied for localized, oral, and parenteral (intraperitoneal or intravenous), and/or device-associated delivery methodologies. Tannic acid has been previously implemented as an anticancer and chemosensitizer [314], also to solubilize various anticancer molecules such as doxorubicin, to prevent adverse side effects of chemotherapy such as cardiotoxicity [315]. In this study, it was found that TA can efficiently bind with PTX molecules in a nanoparticle matrix, giving rise to particle sizes of less than $100 \mathrm{~nm}$ as seen under transmission electron microscopy. The TA matrix core allows PTX to encapsulate within the core, allowing a high encapsulation efficiency of $96.49 \pm 0.43 \%$. A number of investigations suggest that nanoparticle formulations whose particle size is $\sim 100 \mathrm{~nm}$ can be capable of 
penetrating deeply into tumor tissues via the EPR mechanism [316] and negatively charged nanoparticles are associated with higher cellular uptake [317]. Conventional drug delivery approaches have already resulted in FDA approvals for oncology purposes.

Tannic acid is a naturally occurring polyphenol that has other potential benefits, such as being an antioxidant [318], an antiviral [319], an anti-inflammatory, and it also has chemotherapeutic potential. Our strategy of selecting tannic acid relies on the fact that since it is a PARP-1 inhibitor, it can reduce PARP-1 mediated cell death and thus causes $\mathrm{NAD}^{+}$depletion $[315,320]$. Based on this evidence, we developed tannic acid-PTX nanoparticles (TAP NPs) to potentiate chemotherapeutic efficiency. The generated TAP NPs were characterized for particle size in HEPES buffer to mimic the buffering activity in the body and to have clearer insights about the in vitro effects that TAP NPs could encounter (Figure 3-4B). TEM (Figure 3-4C) confirms that the particle size distribution of TAP NPs is in agreement with DLS measurements $(102.22 \pm 14.05 \mathrm{~nm})$. To further confirm that TAP NPs maintain the functional integrity of both tannic acid and PTX, spectroscopic (FTIR), thermogravimetric, and X-ray diffraction characterizations were conducted (Figure 3-4D,F), which confirm the coexistence of both components. Furthermore, a time-dependent increase in cellular internalization of dye-loaded nanoparticles (Figure 3-5A) and enhanced PTX uptake into the intracellular components of cancer cells (Figure 3-5B) were observed, indicating that TAP NPs may overcome P-gp efflux mechanism. This is further supported by P-gp-Glo assay and higher accumulation of RH123 (Figures 3-8A through $\mathbf{C}$ ), both of which confirmed drug efflux was significantly greater in the case of plain drug than with TAP NPs. This confirms tannic acid does have a role in inhibiting efflux by the P-gp transporters located on the surface of the cancer cells, as previously reported $[16,29]$.

To further demonstrate the superior anticancer ability of TAP NPs, an MTT assay was performed (Figure 3-6A). The cytotoxicities of TAP NPs and PTX were compared in both of the $\mathrm{BC}$ cell lines. Significant decreases in the $\mathrm{IC}_{50}$ values were observed with TAP NPs (Figure 36A,B). Similar effects were observed in clonogenic assays (Figure 3-6C,D). There is an impressive development in the treatments for $\mathrm{BC}$, yet metastasis-related recurrence is the major impediment and probably the major reason for BC-related deaths. Thus, therapy resulting in inhibition of migratory signaling is an effective strategy to prevent these circumstances. Our study demonstrates that TAP NPs resulted in an enhanced inhibitory effect on both migratory and invasion properties of BC cells (Figures 3-7A,C). This confirms that TAP NPs not only extend enhanced drug availability for tumor sites but also curb metastasis as well, preventing drug resistance.

PTX causes the stabilization of polymerized microtubules in cancer cells, thus preventing cells from further proliferating. This causes mitotic cell cycle arrest. PTX is reported to inhibit the growth of microtubules in MDA-MB-231, causing cell cycle arrest during mitosis [321]. Our results are consistent with literature reports which clearly show that TAP NPs can prevent drug efflux, which facilitates greater amounts of drugs reaching cancer cells due to the presence of Tannic acid [308]. This is shown by suppression of microtubule mass by TAP NPs, causing cell death probably in prophase itself, compared to free PTX, for which cell morphology suggests that cells treated with free PTX have passed prophase and could have proliferated. Our investigation suggested that TAP NPs were efficiently involved in stabilization of $\beta$-tubulin 
(Figure 3-9A,B) where a significant increase in mitotic arrest is a direct correlation of apoptotic death. An enhanced microtubule polymer mass was elucidated for TAP NPs $10 \mathrm{nM}$ treatment, suggesting the enhanced effectiveness of TAP NPs over that of PTX. Bad is a proapoptotic protein and $\mathrm{Bcl}-\mathrm{xL}$ is an anti-apoptotic protein. Bad counteracts the action of $\mathrm{Bcl}-\mathrm{xL}$, as evidenced by an immunoblotting assay (Figure 3-9C). Cleaved PARP is the hallmark protein that demonstrates apoptosis. Enhanced levels of apoptosis-associated proteins (Cleaved PARP and Cleaved Caspase- 7) lead to the induction of apoptosis as demonstrated with TAP NPs exposure. The comparatively lower expression of Cleaved PARP and Cleaved Caspase-7 with PTX treatment demonstrates the synergistic effect due to the combination therapy of a PARP-1 inhibitor (tannic acid) and PTX. p53 is the tumor suppressor gene that causes cell cycle arrest in the G1 or G2 phase [322]. Literature suggests drug efflux as one of the mechanisms for drug resistance, activating MDR-1 gene due to the drug treatment, which causes overexpression of Pgp, thus inducing chances of a tumor developing drug resistance [323]. Adoption of therapeutic strategies that overcome the MDR phenomenon remains highly sought after in the clinical setting. The self-assemblies of TA and PTX complexes can alter this phenomenon, facilitating the uptake of TAP NPs, which can significantly reduce the drug resistance protein MDR1, as

seen in Figure 3-9C. Similar results were obtained from the heat maps, showing the inhibition of MDR1 and ABC-related proteins. These results explain the profound activity of TAP NPs in contrast to native drug treatments, demonstrating the chemosensitizing ability of our formulation (Figure 3-9D,E). Taken together, the data show that we have developed TAP NPs that have superior anticancer potential, prevent chances of cancer recurrence and drug resistance, and lead to apoptosis.

Altogether, this is successful feasibility and proof-of-concept study that promises the formulation of a universal anticancer molecule through a simple self-assembly process using tannic acid. Using the polyphenolic tannic acid as a shell for the nanoparticle provides not only a potent anticancer moiety but can also prevent cancer recurrence. This simple system resulted in an improved therapeutic outcome in cell-based BC cell line models.

\section{Conclusion}

In summary, tannic acid provides improved PTX self-assembly nanoparticle formation via strong hydrogen bonding over that of ten other polymer excipients tested. This self-assembly approach offers an improved alternative strategy to generate nanoparticles using natural or small molecule excipients such as tannic acid which is classified as an FDA-approved pharmaceutical excipient and is considered safe and can also be utilized to develop a potent yet non-toxic nanoparticle formulation. The formation of TAP NPs was confirmed by characterizing the complexes with FTIR, electronic microscopy, XRD, and TGA analysis. The TAP NPs efficiently internalized into the cytoplasm of MDA-MB-231 cells. TAP NPs mediated delivery of PTX, resulting in superior anticancer (through proliferation and clonogenic assays) and antimetastatic (through migration and invasion assays) profiles over PTX alone. Additionally, TAP NPs induced greater $\beta$-tubulin stabilization in BC cells, ultimately leading to apoptosis. Microarray analysis further suggested a unique superiority of TAP NPs over free PTX in BC treatment that reconfirms our finding of superior activity imparted by TAP NPs. This correlates to the fact that native drug suffers P-glycoprotein mediated drug efflux, which is the root cause of the 
development of Multidrug resistance (MDR) [324]. This lay a solid platform for TAP NPs which can further be implemented in improving current treatment modalities for $\mathrm{BC}$ that possess not only potent anticancer effects but extends therapeutic benefits by minimizing drug efflux [324] and off-target effects, as demonstrated in the graphical representation (Figure 3-10)

These key findings altogether indicate that tannic acid, when used to formulate effective yet nontoxic anticancer nanoparticles with drugs such as PTX, has an excellent potential for translation from the bench to bedside cancer therapy. Our efforts to develop a "first-generation" representative nanoparticle can be further developed and optimized as a targeted nanoparticle formulation for enhanced $\mathrm{BC}$ treatments. Although, nanoparticles are efficacious yet face a high probability of activating the innate immune mechanism which would result in the destruction of the foreign particles. To prevail over this obstacle, active nano-carrier systems are conjugated to biomolecules including ligands and antibodies $[110,111]$. Such strategy could be applied to modify the TAP NPs into even more active nano therapy treatments. 


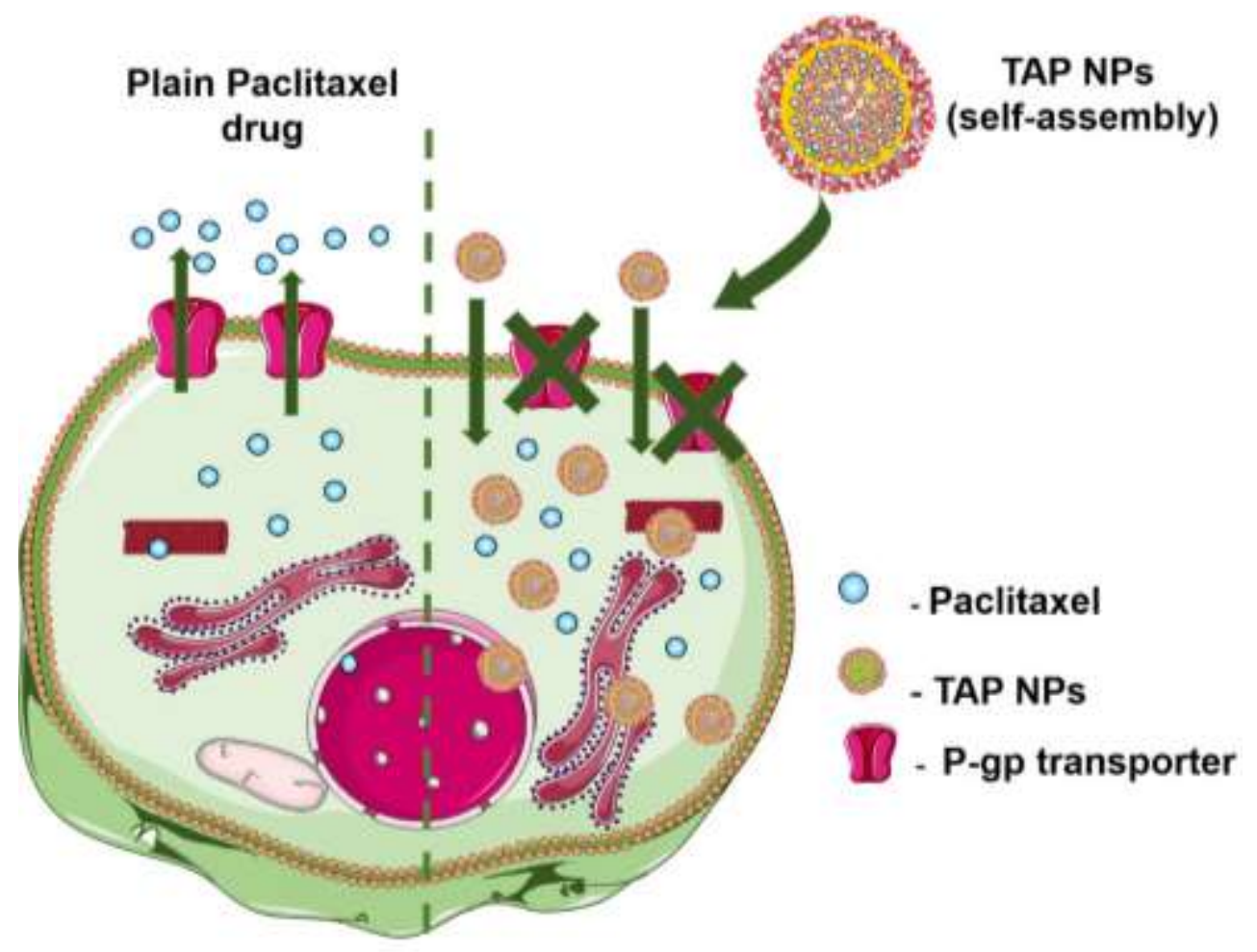

\section{Breast cancer cell}

Figure 3-10. Schematic representation of TAP NPs inhibiting P-gp efflux

TAP NPs are retained longer in the cancer cells due to the presence of TA, which is an inhibitor of P-gp efflux transporter preventing TAP NPs from getting efflux out of the cell membrane by the P-gp transporters. However, native drug PTX is effluxed out from the cells due to the innate nature of P-gp transporters that tend to expel chemotherapeutic agents. Thus, TAP NPs extend better anticancer efficacy over native drug. 


\section{CHAPTER 4. BIO-MEMBRANE INSPIRED NANOPARTICLES FOR ENHANCED THERAPEUTIC EFFICACY IN TNBC}

\section{Introduction}

$\mathrm{BC}$ remains the second most commonly diagnosed cancer among women in the United States after skin cancer [325], with about $12 \%$ probability for women to develop BC in their lifetime. The estimated deaths for 2019 from BC are about $16 \%$ of the total cases with metastatic $\mathrm{BC}$ being the cause of $90 \%$ of the deaths [326]. Although there have been significant advances in the treatment of $\mathrm{BC}$ which has provided survival benefits to patients, yet the survival statistics of the metastatic $\mathrm{BC}$ hinders clinical benefits, due to the aggressive nature that proliferates and advances to multiple sites [327]. Currently, the mainstay treatment for metastatic BC remains systemic administration of therapeutic agents based on the specific subtype they belong to, such as hormone therapy, chemotherapy by cytotoxic agents such as anthracyclines, taxanes, and platinum-based compounds [328]. These chemotherapeutic agents are delivered systemically with the expectation that at least a fraction of the dose would be reaching the metastatic sites, thus demonstrating poor bioavailability. However, these agents demonstrate anticancer benefits, yet the median survival is $\leq 24$ months [329], with a $27 \%$ relapse within 5 years survival rate and these treatments often are followed by relapses, recurrence, and other adverse toxicities. Among these cytotoxic agents PTX is the most commonly administered taxane and is used as the firstline treatment for metastatic BC. PTX is formulated as Taxol ${ }^{\circledR}$ with Cremophor EL and ethanol and exerts serious adverse effects such as sensory axonopathy and ganglio-neuropathy when administered in combination with cisplatin [330]. It is also known to induce neutropenia, severe peripheral neurotoxicity which often ranges from $30 \%$ to $40 \%$ of patients receiving chemotherapy [331]. Other risk factors include arthralgias and myalgias [332] and even diabetes mellitus [121]. The advancement of a nanotechnology-based drug delivery system has made significant improvements in overcoming conventional limitations.

Nanoparticles based drug delivery system offers enhanced permeation and retention (EPR) effect that renders them to penetrate the leaky tumoral neovasculature allowing nanoparticles of poorly soluble drugs to circulate longer, deliver chemotherapeutics specific site [333] and improve the tolerability of cytotoxic agents [334]. With this respect, Abraxane, an albumin-bound PTX nanoparticle formulation (nab-PTX) was developed to reduce toxicity issues of Taxol ${ }^{\circledR}$ and have shown $\sim 2$-fold improvement in the overall response rate of patients $[191,199]$ in contrast to PTX or Taxol ${ }^{\circledR}$. However, despite the therapeutic advantages extended by nanoparticles, delivery to the metastatic and advanced stage $\mathrm{BC}$ remains a major hindrance due to the clearance by reticular endothelial system (RES) which produces subtherapeutic concentration for tumor penetration or due to the presence of dense extracellular matrix [297]. To improve tumoral delivery active targeting by attaching ligands such as polymers (poly (ethylene glycol) or pluronics), cationic peptides, aptamers, and antibodies on nanoparticles have been employed $[335,336]$. This strategy provides active targeting to the receptors that are discretely unregulated on cancer cells thus allowing receptor-mediated endocytosis that provides cell-specific adherence and uptake of nanoparticles at specifically to target site [337-339]. Decoration with cationic or polymeric moieties causes immunogenicity issues probably caused by the recognition of surface charged protein by RES [297, 340]. Most of the studies that are 
"actively targeting" mainly involve coating the surface with these moieties that offer the advantage of selectivity yet is mainly harbored around the idea of EPR effect, resulting in targeting only the primary tumor, with little or no attention given to the metastatic area. Thus, new strategies are in dire need that not only targets the primary tumor but also the metastasis tumor microenvironment.

Among all the new strategies employed for targeted therapy, one such strategy is the functionalization of particles with bio-inspired artificial cell membranes or naturally derived cell membranes. The advantages are myriad, because of the utilization of naturally occurring cells they are intrinsically biocompatible, biodegradable, and nonimmunogenic [341]. Additionally, the presence of proteins, glycan, and sialic acid moieties on the natural cell membrane serves as a receptor/ligand for receptor-mediated endocytosis or for suppressing immune attack [342]. Thus, with this aim, we have isolated cell membranes from natural cells with specific "self-marker" expression on them that allows them to be internalized not just at the primary tumor site but also to the metastatic regions. Rationalizing from our preliminary and research findings, we hypothesize this approach will target the primary tumor and the metastatic tumor microenvironment with minimal adverse effects on healthy cells, due to cell-specific binding to the antigen-receptor on aggressive and metastatic TNBC. We believe this strategy can extend unique, personalized nanomedicine with non-immunogenicity and therapeutic superiority for targeting metastatic BC. Our preliminary results show activated human neutrophil membrane shows enhanced intercellular uptake by TNBC over normal breast epithelial cells at a particular concentration. Our initial findings suggest, PVP [287] and tannic acid prevents potential P-gp efflux [343]. Thus we used these two excipients to generate PVT (Polyvinylpyrrolidone-Tannic acid self-assembled) nanoparticles after evaluating their enhanced efficacy in TNBC cells [300]. To testify our hypothesis, we used polyvinylpyrrolidone (PVP) nanoparticles and tannic acid to form the core for the nanoparticles that also provide anticancer effects, as demonstrated in our previous study, such as prevents drug efflux [344]. Therefore, the aim of the study was to develop and characterize PVT nanoparticles coated with cell membranes isolated from human neutrophil, along with membranes from four more different natural cells (Natural killer:NK-92, Mesenchymal stem cells: MSC, Jurkat: JUR and RAW264.7:RAW) and screen the best candidate for targeting TNBC, both in in vitro and in vivo models.

\section{Materials and Methods}

\section{Chemicals and Solvents}

Tannic acid (catalog no. 403040), PVP (MW 40,000), TMA Cores: tissue and cell microarrays (Catalogue no. BR243v) were procured from US Biomax. Inc, 96 low attachment plates were procured from Corning, HEPES (catalog no. H3375) were purchased from Aldrich (St. Louis, MO, USA). All chemicals, reagents, and solvents were used as received without further purification. 


\section{Cell Culture}

Humanized BC cell lines (MDA-MB-231, MDA-MB-468, and MCF-7) and NK-92, Mesenchymal Stem Cells, Jurkat, RAW264.7 were purchased from American Type Culture Collection (ATCC, Manassas, VA, USA). Human Neutrophils were procured from Astarte Biologics that are tested for bloodborne pathogens HIV-1 and 2, Hepatitis B, Hepatitis C, and HTLV-1 to ensure safety. Breast cell lines were cultured in Dulbecco's Modified Eagle Medium (DMEM) that was supplemented with phenol red, 10\% fetal bovine serum, $100 \mathrm{U} / \mathrm{ml}$ penicillin and $100 \mathrm{U} / \mathrm{ml}$ streptomycin (Thermo Fisher Scientific, Grand Island, NY). Mammary Epithelial Cell (MCF10A) was gifted by our generous collaborator Dr. S. C. Chauhan laboratory and was cultured with MEGM ${ }^{\mathrm{TM}}$ Mammary Epithelial Cell Growth Basal Medium (Catalogue No. CC3151) with supplements and growth factors (Catalogue no. CC-4136). For all experiments, cell lines were incubated at $37^{\circ} \mathrm{C}$ in a humidified 5\% CO2 and 95\% air atmosphere (Thermo Fisher Scientific, Waltham, MA, USA). At 80-90\% confluency, these cells were trypsinized, counted using Trypan Blue $(0.4 \%)$ and seeded as per specific experiments. Cells were allowed to adhere to the plate surface overnight before implementing any further treatment.

\section{Activation and Isolation of Cell Membrane}

Neutrophil membrane (NEU) was activated using LPS (100 $\mathrm{ng} / \mathrm{ml})$ added to the culture medium for 4 hours to ensure activation, followed by a wash in PBS. The rest of the cells (NK/ MSC, JUR, RAW) were extracted without any activation. To isolate the plasma membranes, cells are suspended in Isolation Buffer composed of mannitol, sucrose, BSA, EDTA, and Tris$\mathrm{HCl}$ and supplemented with protease inhibitor cocktail (PCI). Cells were homogenized in Dounce homogenizer, centrifuged at $800 \times \mathrm{g}$ at $4^{\circ} \mathrm{C}$ for $10 \mathrm{mins}$ and the pellet discarded to remove the unbroken cells and nuclei. The supernatant was centrifuged at $10,000 \times \mathrm{g}$ at $4^{\circ} \mathrm{C}$ to remove the mitochondria. The supernatant was ultracentrifuge using Sorvall WX Ultra 80 (Thermo Scientific) again at $100,000 \times \mathrm{g}$ at $4^{\circ} \mathrm{C}$ for 1 hour to pellet the membranes. Finally, the plasma membrane pellet was suspended in Tris- $\mathrm{HCl}$ and EDTA and then stored at $-80^{\circ} \mathrm{C}$ for later use, to maintain the membrane integrity freeze-thaw cycles were prevented by aliquoting membranes in smaller batches. Protein estimation was quantified using the BCA protein assay.

\section{Preparation of Polyvinylpyrrolidone-Tannic Acid (PVT) and Membrane-Coated (PVT- MEM) Nanoparticles (NPs)}

Nanoparticles were prepared by the self-assembly technique and is followed by solvent evaporation. Briefly, $2.5 \mathrm{mg}$ PVP and TA were dissolved in $0.5 \mathrm{ml}$ aqueous medium separately and then mixed in 1:1 ratio in $8 \mathrm{ml}$ glass vial under continuous stirring at $400 \mathrm{rpm}$ on a stir plate. Now, $100 \mu \mathrm{l}$ of PTX dissolved in acetone $(1 \mathrm{mg} / \mathrm{ml})$ was dropwise added and the speed increased to $900 \mathrm{rpm}$. They were stirred overnight to ensure the acetone was evaporated to finally obtain PVT nanoparticle solution. The membrane-bound nanoparticles (PVT-MEM NPs) were prepared by simple sonication technique. Based on the BCA assay, the protein concentration(s) were determined for each of the different cell membrane(s). For the synthesis of PVT-MEM NPs, PVT NPs were mixed to each of the extracted MEM, at a concentration equivalent to $16: 1$ and 
allowed to stir at $4{ }^{\circ} \mathrm{C}$ for 24 hours. The next day, the mixture was sonicated at $100 \mathrm{w}$ for 30 secs with intermittent cooling on ice, followed by centrifugation at $14000 \mathrm{rpm}$ at $4^{\circ} \mathrm{C}$ for $10 \mathrm{mins}$, to remove the unbound membranes. All batches were freshly prepared before experimental use to maintain the membrane integrity.

\section{Characterization of PVT and PVT-MEM NPs (PVT-NEU, PVT-NK, PVT-MSC, PVT- JUR, PVT-RAW)}

\section{Particle size and zeta potential}

The average particle size, particle size distribution (PDI) and zeta potential of our developed PVT and all the five PVT-MEM NPs were measured by the dynamic light scattering (DLS) principle using Zetasizer (Nano ZS, Malvern Instruments, Malvern, UK). Briefly $50 \mu 1$ of freshly prepared formulation was dispersed in deionized water and was probe sonicated (VirSonic Ultrasonic Cell Disrupter 100, VirTis) for 30 secs to ensure formation of homogeneous nanoparticle solution and minimize the nanoparticle aggregation. Particle size measurements were performed in water at $25^{\circ} \mathrm{C}$ and an average diameter of particle size distribution were determined from three runs. Zeta potential of the samples were measured using 1X phosphate-buffered saline (PBS). The zeta potential of nanoparticle formulations was also based on the average of 3 readings (each reading $=30$ runs).

\section{Short-term stability study}

The stability of the different PVT and PVT-MEM NPs were assessed at three different temperature condition $4,25,37^{\circ} \mathrm{C}$ using DLS particle size analysis. Briefly, samples were freshly prepared and incubated at three respective conditions for 10 days and the change in particle size with respect to time is assessed. Particle size of samples were measured in freshly prepared 1 mM of HEPES buffer using HEPES salt in ultrapure water, supplemented with PCI to prevent precipitation, as mentioned in section 2.5 .

\section{Spectral analysis}

Fourier transform infrared (FTIR) spectra was acquired using Universal ATR sampling Accessory plate using a Spectrum 100 FTIR spectrophotometer (Perkin Elmer, Waltham, MA) to confirm the structural integrity of the cell membranes inside the nanoparticles. For this, PVT and all the five PVT-MEM NPs were prepared and lyophilized using a Labconco Freeze Dry System $\left(-48^{\circ} \mathrm{C} 133 \times 10^{-3} \mathrm{~m}\right.$ Bar; Labconco, Kansas City, MO, USA) to obtain lyophilized powder. These samples were placed on the tip of the ATR objective to obtain the spectra between 4000 and $650 \mathrm{~cm}^{-1}$ at a scanning speed of $4 \mathrm{~cm}^{-1}$ for 32 scans. The FTIR spectra is presented as \%T vs. $\mathrm{cm}^{-1}$ and analyzed using Spectrum 100 software. 


\section{Transmission electron microscopy}

To confirm the size obtained from the DLS and to investigate the structural morphology of PVT and PVT-MEM NPs especially to view the cell membrane coating around the formed nanoparticles, we imaged using JEOL 200EX transmission electron microscopy (TEM) (JEOL Ltd, Tokyo, Japan) operating at $80 \mathrm{kV}$. Freshly prepared samples were sonicated vigorously to prevent any agglomeration of NPs and $20 \mu \mathrm{l}$ was slowly placed on the shiny side of the 150mesh standard TEM grid (Electron Microscopy Sciences, PA, USA). It was negatively stained using $2 \% \mathrm{w} / \mathrm{v}$ uranyl acetate solution. The excess amount of stain was wicked off and the grid was allowed to air dry, followed by imaging using an AMT camera at a direct magnification of 100,000X under the TEM.

\section{Identification of Membrane-Associated Proteins}

The presence of membrane-associated proteins on the particles was verified by Coomassie blue staining. For this, the NEU extracted from neutrophils, as mentioned in section 2.4, were collected. They were further coated on the top of the PVT nanoparticles as described in the previous section (Preparation of PVT NPs). After both the NEU and PVT-NEU were obtained, a short spin on a benchtop centrifugation at a high speed of $14000 \mathrm{rpm}$ for $30 \mathrm{secs}$ at $4^{\circ} \mathrm{C}$, was done to remove the unbound NEU from the surface of the PVT nanoparticles. For the analysis by Coomassie blue-SDS PAGE, the samples were processed as mentioned in Kang et. al [71]. Briefly, samples were lysed and centrifuged at $13,000 \times \mathrm{g}$ for $5 \mathrm{mins}$ at $4^{\circ} \mathrm{C}$. The protein concentration was quantified using a standard BCA assay. Followed by mixing the supernatant protein with SDS loading buffer, heated to denature the proteins and an equivalent of $20 \mu \mathrm{g}$ of protein (equivalent to the NEU and in PVT-NEU) per sample was loaded in a $10-20 \%$ SDSPAGE. After the sufficient run time, the protein gels were stained Coomassie blue fast staining solution and imaged using the ChemiDocTM MP System (Bio-Rad, Hercules, CA, USA).

\section{In Vitro Cellular Uptake}

To determine the preferential targeting of membrane coated nanoparticles, intercellular uptake of PVT and all the five PVT-MEM NPs were conducted in BC cells (MCF7, MDA-MB468, and MDA-MB-231) and breast epithelial cells (MCF10A), which was used as an experimental control. For this experiment, C6 was loaded instead of PTX in PVT and PVTMEM NPs and was constructed similarly (Preparation of PVT NPs). In brief, all the different types of cells were seeded at $0.5 \times 10^{6}$ cells in a 12 -well plate and allowed to adhere to the plate surface overnight. Next day, dye equivalent to $5 \mu \mathrm{g}$ C6 loaded PVT and PVT-MEM NPs were treated for 3 hours to ensure the stability of $\mathrm{C} 6$ and such that it does not leach out of the nanoparticles but also giving enough time for the cells to intracellularly uptake the dye an incubation time of 3 hours was selected. After the incubation time cells were washed thrice with 1X PBS to remove the C6 bound o the outer cell surface. To further process the cells, we choose to use DMEM phenol red-free medium to reduce the auto-fluorescence. For qualitatively images, cells were imaged using EVOS® FL Imaging System (AMF4300, Life Technologies, Carlsbad, CA, USA) and quantitative analysis was done after injecting samples into an Accuri C6 Flow 
Cytometer (Accuri Cytometer, Inc., Ann Arbor, MI, USA). Quantitative uptake of C6 was acquired using the fluorescence levels in FL1 channel (488 excitation, Blue laser, $530 \pm 15 \mathrm{~nm}$, FITC/GFP). Average reading and standard error of mean were calculated from 3 runs. Data reported as normalized mean fluorescence value (after normalizing all values with respect to control values, for the respective cell lines).

\section{Evaluation of In Situ Uptake}

For this, tumor tissues of various grades and normal breast adjacent epithelial tissue sections were analyzed for cellular uptake of C6 and imaged by EVOS $®$ FL Imaging System (AMF4300, Life Technologies, Carlsbad, CA, USA. Briefly, tissue and cell micro were procured, and a study was conducted similarly as immunohistochemistry (IHC). Slides were deparaffinized, rehydrated by different concentrations of alcohol content followed by antigen retrieval. $100 \mu \mathrm{g} / \mathrm{ml} \mathrm{C6}$ equivalent, loaded in PVT and PVT-MEM NPs that were freshly prepared were used to stain the slides using a PAP pen for an overnight, followed by three consecutive washes of 5 mins each and imaged. All images will be captured using the same laser power and gain settings to ensure comparability for intensity analysis. Fluorescent intensities will be quantified using Image J. The corrected total cell fluorescence (CTCF) was measured using the Equation 4-1. This was done to normalize the actual fluorescence inside the cells, independent on the size and structure of each cells [345].

$$
\begin{aligned}
& \text { CTCF = Integrated Density - (Area of selected cell * } \\
& \text { Mean fluorescence of backgrou nd readings) (Eq. 4-1) }
\end{aligned}
$$

\section{Biological Assays}

\section{Cell viability assay}

Cell viability assay was assessed using MTT assay on TNBC cell line: MDA-MB-231 and as non-TNBCBC: MCF7 was used. Briefly, $5 \times 10^{3}$ cells were dispersed in $100 \mu \mathrm{lmedia} /$ well in 96-well culture plates were seeded and allowed to stand overnight. The next day these cells were treated with 0-20 nM equivalent PTX or PVT/PVT-MEM NPs for 48 hours. Cell lines with no treatment served as experimental controls. After the required incubation time, $20 \mu \mathrm{l}$ of MTT reagent solution was added and incubated for $2-3$ hours at $37^{\circ} \mathrm{C}$. After formazan crystals are formed the media was aspirated out such that, not to disturb the formazan crystals and $100 \mu 1$ of DMSO were added to dissolve the salt formation Absorbance reading was recorded at $490 \mathrm{~nm}$ using a Microplate Reader (BioTeK Cytation 3, Winooski, VT, USA). Cell proliferation with respect to control cells was calculated. All the experiments were performed at least in triplicates. Cell morphology changes with respect to treatment were further confirmed by imaging using EVOS ${ }^{\circ}$ FL Imaging System at 20X magnification. 


\section{Cell migration}

Boyden's chamber cell migration assay was used to confirm migration by the PVT and PVT-MEM NPs in TNBC cells (MDA-MB-231). Briefly, $0.5 \times 10^{5}$ cells were suspended in serum-free media along with the equivalent amount of $5 \mathrm{nM}$ of PTX or PVT/PVT-MEM NPs, added to the upper chamber of the 96-transwell inserts (catalogue no.3374, Corning, NY, USA). Cells receiving no treatment were used as an experimental control. These inserts were then placed inside the 96-well plates in 10\% FBS media. After 24 hours, the migrated cells at the lower inside of the Transwell inserts were fixed using 4\% formaldehyde and then further stained using crystal violet. The cells on the upper side of the insert are removed using a cotton swab. Inserts were then imaged using the EVOS $®$ FL Imaging System.

\section{Cellular invasion}

BD Biocoat Matrigel Invasion Chambers (BD Biosciences) was used to study the invasion assay. Briefly, MDA-MB-231 $\left(3.5 \times 10^{4}\right.$ cells $)$ were seeded in the matrigel invasion chambers in serum-free medium and treated with equivalent amount $5 \mathrm{nM}$ of PTX or PVT/PVTMEM NPs for 24 hours. The lower chambers were placed with 10\% FBS medium which served as a chemoattractant. The cells that were able to invade were fixed with methanol and then stained using crystal violet. The plates were imaged using EVOS® FL Imaging System

\section{Clonogenic assay}

Colony-forming assay was performed to investigate the proliferation ability of on BC cells to form colonies as an in vitro cell survival assay based on the ability of TNBC (MDA-MB$231)$ to grow into the form of a colony. For this study, BC cells were seeded ( 250 cells/well) in a 12-well plate and treated with $5 \mathrm{nM}$ of PTX or PVT/PVT-MEM NPs and set in the incubator for 15 days with intermittent media change. Visible colonies were fixed, stained with hematoxylin and imaged.

\section{D Spheroid assay}

Cells were seeded in an ultra-low 96 well attachment plates with 1000 cells/well plated, followed by treatment with equivalent concentrations $5 \mathrm{nM}$ of PTX or PVT/PVT-MEM NPs for 10 days. The cells were allowed to grow for 7 days and then centrifuged at 200 RCF for 5 mins and further incubated in standard cell culture conditions for 10 days. Growth rate was monitored by imaging the spheroid on from day 0-10 using brightfield microscopy of EVOS ${ }^{\circ}$ FL Imaging System. The growth rate was reported in terms of volume ratio, where first the spheroid volume was determined using Equation 4-2A. The tumor volume ratio (\%) was calculated using

Equation 4-2B.

$$
\begin{aligned}
& V=(\pi x d \max x d \min ) / 6 \text { for } 0,3,7 \text { and } 10 \text { days (Eq. 4-2A) } \\
& \text { Tumor volume ratio }(\%)=\left(\text { Vday }_{n} / \text { Vdayo }\right) \times 100 \text { (Eq. 4-2B) }
\end{aligned}
$$


where $\mathrm{Vday}_{\mathrm{n}}$ is the spheroid volume at the nth day (day $0,3,7$ or 10) of treatment, and $\mathrm{Vday}_{0}$ is the spheroid volume on day 0 prior to treatment.

\section{In Vivo Evaluation of PVT and PVT-MEM NPs}

\section{Tumor xenograft and imaging study}

To grow the orthotopic xenograft mice model we purchased six-week-old female athymic nude mice (nu/nu) from Jackson laboratory (Bar Harbor, ME USA)) and maintained in a pathogen-free environment. All animals were housed and maintained according to the recommendation of the Association for Assessment and Accreditation of Laboratory Animal Care (AAALAC) guidelines. All studies were conducted using UTHSC Institutional Animal Care and Use Committee (UTHSC-IACUC) protocol. To establish orthotopic xenograft tumors in mice, MDA-MB-231 cells $\left(2 \times 10^{6}\right.$ cells) were suspended in phosphate buffer saline (PBS) and Matrigel (BD Biosciences) solution in 1:1 ratio and then injected subcutaneously on the hind flanks of each mice. Tumor volume in mice was monitored using a digital Vernier caliper and calculated using the Equation 4-3.

\section{Ellipsoid formula for tumor volume $(\mathrm{mm} 3)=\pi /(6 \times L \times W \times H)$ (Eq. 4-3)}

When tumor volume reached $\sim 200 \mathrm{~mm}^{3}$, mice were randomly divided (five mice per group) into respective groups and treated with control (PBS treated), PTX (10 mg/kg), PVT and PVT-NEU NPs (PTX equivalent to $10 \mathrm{mg} / \mathrm{kg}$ ) for 4 weeks, given intraperitonally, twice a week. For imaging, PVT and PVT-MEM NPs were loaded with Indocyanine dye (ICG) for in vivo imaging ( 3 mice per group), similarly loaded inside the nanoparticles as mentioned in section 2.4. For this, tumors were grown in similar fashion MDA-MB-231 cells injected in the hind flank and tumor size reaching $200 \mathrm{~mm}^{3}$ mice were randomly assigned into two treatment groups: 100 $\mu \mathrm{g} / \mathrm{ml}$ of ICG-labeled PVT or PVT-MEM NPs, plain ICG solution was used as an experimental control, given intraperitoneally. After injecting the NPs once, all mice were imaged at 3, 6, 24, 48 and 72 hours to find the fate of the NPs and retention in the body using the XRMS Imaging System (Caliper Life Sciences, Waltham, MA) equipped with a coupled device camera (CCD) housed at the UTHSC core facility was used. These animals were fed with a special diet of Teklad Global Rodent Diets (Envigo, Huntingdon, United Kingdom) to avoid background autofluorescence from the diet, 24 hours before the initiation of the imaging study. Mice were anesthetized with $2 \%$ isoflurane and imaged in immunofluorescence intensity (integration: 30 secs; binning: 4). The peak immunofluorescence signal intensity is calculated by the sum of all detected photon counts within the region of interest (ROI) after subtraction of background fluorescence, in photon $/ \mathrm{s} / \mathrm{cm}^{2} / \mathrm{sr}$. Biodistribution of ICG was examined by assessing ROI of excised heart, lungs, liver, kidney and spleen along with tumor retention. After the necessary time, mice were euthanized, and organs excised. Mice were euthanized either when the tumor volume reached $\sim 1200 \mathrm{~mm}^{3}$ or the mice weight lost was more than $10 \%$ body weight. Mice were sacrificed by exposing them in the $\mathrm{CO}_{2}$ incubator and cervical dislocation. Tumors were excised and processed for tissue sections, histopathology and hemo toxicity images. Blood was collected after post-mortem cardiac puncture and submitted to Idexx Bioresearch for histopathology and blood chemistry analysis for complete blood cell count analysis. Also, hepatotoxicity was 
assessed by measuring serum levels of alanine aminotransferase (ALT), aspartate aminotransferase (AST) enzymes and total bilirubin (TBIL) and kidney function marked by blood urea nitrogen (BUN) levels.

\section{Immunohistochemistry}

The effect of PVT and three different PVT-MEM NPs were determined for expression of BAK, BCL2, $\beta$-tubulin III isoforms, Cleaved caspase 3, PCNA. Standard IHC protocol was followed [346], tumor tissues were deparaffinized, rehydrated, soaked with $0.3 \%$ hydrogen peroxide and processed for antigen retrieval. After blocking with background sniper, the samples were subjected to staining for expression of respective proteins expression on the tissues. The slides were counterstained with hematoxylin, dehydrated, mounted Prolong Diamond Antifade Mountant (catalog no. P36961). The histological difference in samples was analyzed by H\&E staining to study organ toxicity.

\section{Statistical Analysis}

All data were generated and analyzed using GraphPad Prism 5.03 Software (GraphPad Software, San Diego, CA) and results are presented as mean \pm standard error of mean (SEM). Statistical significance was performed using a student's t-test analysis when comparing between two groups. When comparing the statistical significance among multiple treatment groups oneway ANOVA post-hoc Tukey HSD test was performed, or for estimating the statistical difference between groups with multiple variable and multiple treatment groups, two-way ANOVA Bonferroni posttest. A p-value $\leq 0.05$ was considered significant.

\section{Results}

\section{Preparation and Characterization of PVT and PVT-MEM NPs (PVT-NEU, PVT-NK, PVT-MSC, PVT-JUR and PVT-RAW)}

To preparation of PTX loaded polyvinylpyrrolidone-tannic acid (PVT) and membranecoated (PVT-MEM) nanoparticles (NPs) were processed in four steps. Firstly, it was pivotal to ensure the cells used (neutrophils, Natural Killer cells, mesenchymal stem cells, Jurkat cells and RAW 264.7 cells) preserves the cellular membrane functions of the source cells. Literature suggests [342] neutrophils needs to be activated by lipopolysaccharide (LPS), a TLR-4 ligand that induces inflammatory responses in neutrophil, which in the process would endow maximal adhesion ability to the extracted neutrophil cell membranes (NEU). For the same purpose, neutrophils were LPS activated for 4 hours to induce an inflammatory response for the proper activation of the adhesion molecules on the surface of NEU. Secondly, extraction of the membranes (MEM) from the cells took place, the rest of the cells except neutrophils were used directly from culture condition, after harvesting them in their respective culture media. This was followed by the construction of the core nanoparticle loaded with PTX (for in vitro imaging C6 was loaded, and for in vivo imaging ICG was loaded) by solvent evaporation technique, as 
previously described. Lastly, the coating of the extracted MEM on the surface of the PVT NPs, to yield PVT-MEM NPs was done by mixing them together and subjecting them to sonication to prevent agglomeration of particles and centrifugation to remove the excess of the unbound membranes. From our preliminary studies, we have established a better intracellular uptake occurs at 16:1 for PVT-NEU NPs, which is why we maintained this ratio for the synthesis of PVT-MEM NPs for all the five PVT-MEM NPs throughout this study. The hydrodynamic mean particle size $(\mathrm{nm})$ of PVT and all the five PVT-MEM NPs were determined by dynamic light scattering (DLS) (Figure 4-1A). There was a slight increase in the size after the coating of the MEM, where PVT NPs (nanoparticles without any membrane coating) increased from $220.53 \pm 12.35 \mathrm{~nm}$ to $243.3 \pm 4.65,253.36+5.20,341.06 \pm 44.44,269 \pm 32.23,231.96 \pm 16.03 \mathrm{~nm}$ after coating with NEU, NK, MSC, JUR, RAW respectively. Although there was no statistical significant difference in the particle size among all the PVT-MEM NPs in comparison to uncoated NPs (PVT NPs), there was a slight increase observed in PVT-MSC NPs. The zeta potential of PVT was $-2.89 \mathrm{mV}$ and the addition of the negatively charged cellular membrane causes the zeta potential to increase resulting in $-4.26,-5.54,-4.84,-4.53,-4.11 \mathrm{mV}$, for the five PVT-MEM NPs, respectively (Figure 4-1B), suggesting the successful coating of the cell membranes on the surface of the nanoparticles. Short term stability study (Figure 4-1C) was conducted to confirm the stability of the formulation especially due to the presence of cell membrane proteins, which may tend to agglomerate. Although, there was no significant changes in size of PVT-MEM NPs seen for 5 days after storing them at 4 or $25^{\circ} \mathrm{C}$. However, a long-term stability study needs to be warranted.

Additionally, FTIR spectra (Figure 4-2A) for the cell membranes based nanoparticles (PVT-MEM NPs) due to the presence of lipid bilayer has presence of large number of amide groups present and are characteristics of proteins to have band at 1638 due to $\left(-\mathrm{CO}-\mathrm{NH}_{2}\right)$ [347]. FTIR spectra of PVT NPs demonstrate distinct peaks of N-H stretching at $3258 \mathrm{~cm}^{-1}$ and C $=\mathrm{O}$ stretching at $1715 \mathrm{~cm}^{-1}$ are characteristic PTX peaks [348] which are consistently present in all the five PVT-MEM NPs, demonstrating the proper inclusion of PTX inside the core of the nanoparticles. Additionally, characteristic peaks at $1638 \mathrm{~cm}^{-1}$ due to stretching of conjugated $\mathrm{C}=\mathrm{O}, 1292 \mathrm{~cm}^{-1} \mathrm{C}-\mathrm{N}$ and $\mathrm{C}-\mathrm{O}$ at $1198 \mathrm{~cm}^{-1}[349,350]$ confirms presence of TA and PVP in the nanoparticles. The infusion of the membranes on the surface of the PVT NPs was further confirmed by TEM (Figure 4-2B) which displays the core shell structure of the PVT-MEM NPs that encapsulates the PVT NPs in the core and the surrounding MEM layer in the shell. To ensure that the expression of the membrane proteins was successfully translocated on the surface of the PVT nanoparticles, Coomassie blue-SDS-PAGE analysis was conducted (Figure 4-2C).

\section{Intracellular and In Situ Uptake and In Vitro Proliferation, Migration, Invasion and Colony Forming Assay in BC Cells}

The intracellular uptake and distribution in different type of BC cells and breast epithelial cells were determined. For this, the core shell structure of all the membrane coated nanoparticles (PVT-NEU, PVT-NK, PVT-MSC, PVT-JUR and PVT-RAW) along with PVT used as the respective control were synthesized together and fluorescently labeled with C6. A 3-hours incubation time with the dye loaded nanoparticles, after normalizing the mean fluorescent 
Figure 4-1. Characterization of PVT and PVT-MEM NPs (PVT-NEU, PVT-NK, PVTMSC, PVT-JUR and PVT-RAW)

A) Dynamic light scattering measurement for PVT and PVT-MEM NPs in water using $50 \mu 1$ of freshly prepared samples were added to $1 \mathrm{ml}$ of ultrapure water and probe sonicated for 30 secs and measured using Zetasizer (Nano ZS, Malvern Instruments, Malvern, UK) at $25^{\circ} \mathrm{C}$. PVT NPs exhibited particle size of $220.53 \pm 12.35 \mathrm{~nm}$. There was a slight increase in size after addition of cell membrane bilayer, with $243.3 \pm 4.65,253.36+5.20,341.06 \pm 44.44,269 \pm 32.23,231.96 \pm 16.03$ $\mathrm{nm}$, respectively. Data presented as mean + standard error of the mean $(\mathrm{n}=3)$. B) Zeta potential was measured in $1 \mathrm{X}$ PBS and average of 3 readings (each reading $=30$ runs). PVT NPs had a charge of $-2.89 \mathrm{mV}$, causing electrostatic repulsion between the nanoparticles and the negatively charged bilayers, resulting in zeta potential of $-4.26,-5.54,-4.84,-4.53,-4.11 \mathrm{mV}$ respectively. C) Change in particle size was evaluated by measuring particle size in water as previously described for 10 days, at three different temperature conditions, 4,25 and $37^{\circ} \mathrm{C}$. Data presented as mean + standard error of the mean $(n=3)$. 

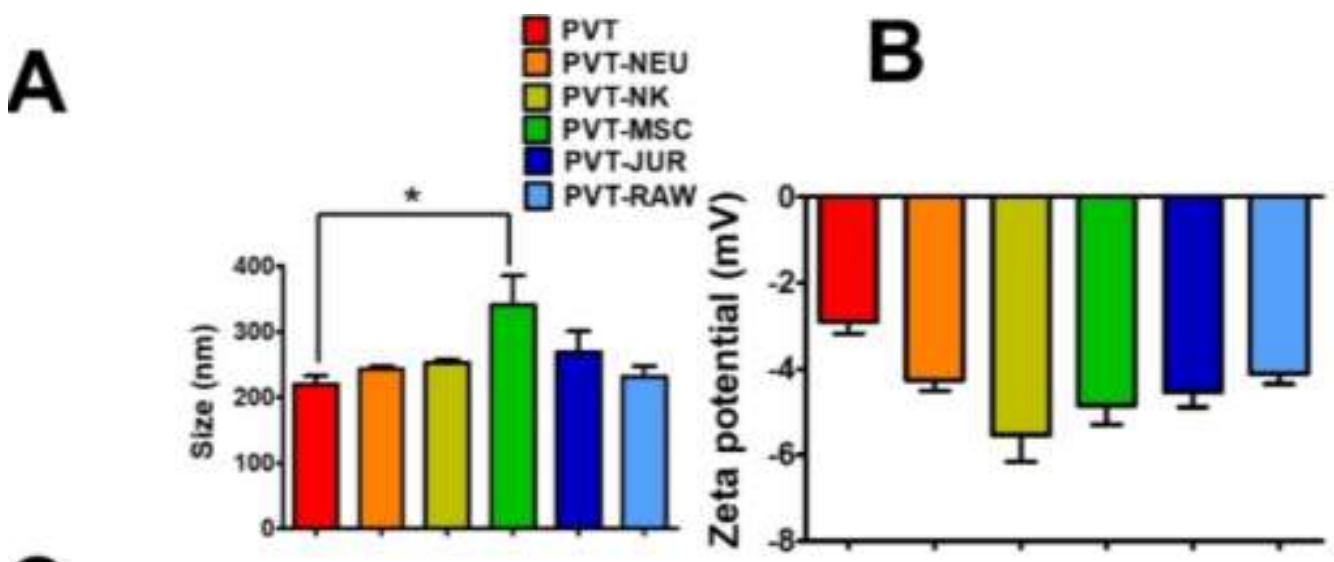

Day 1

Day 2
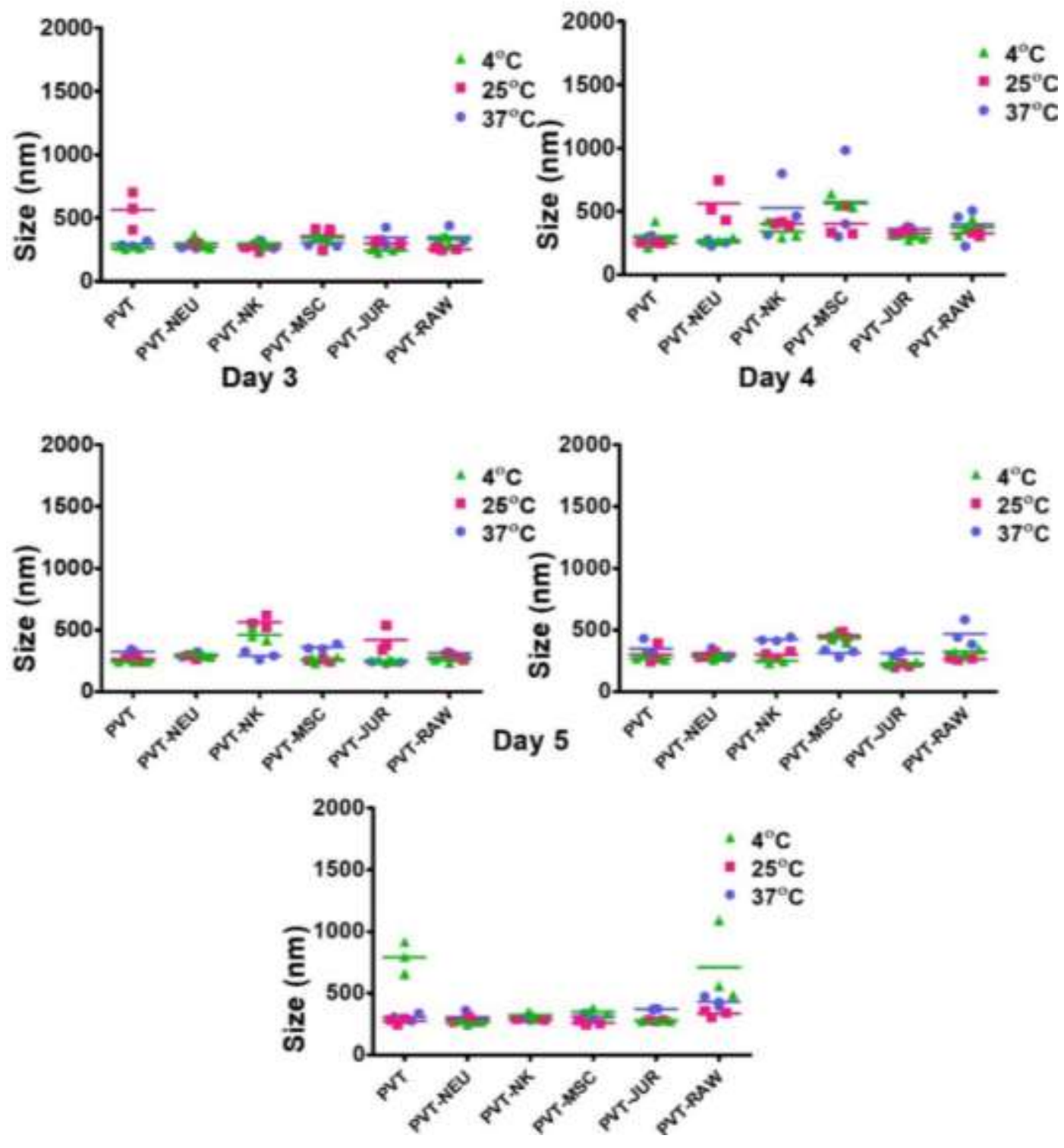

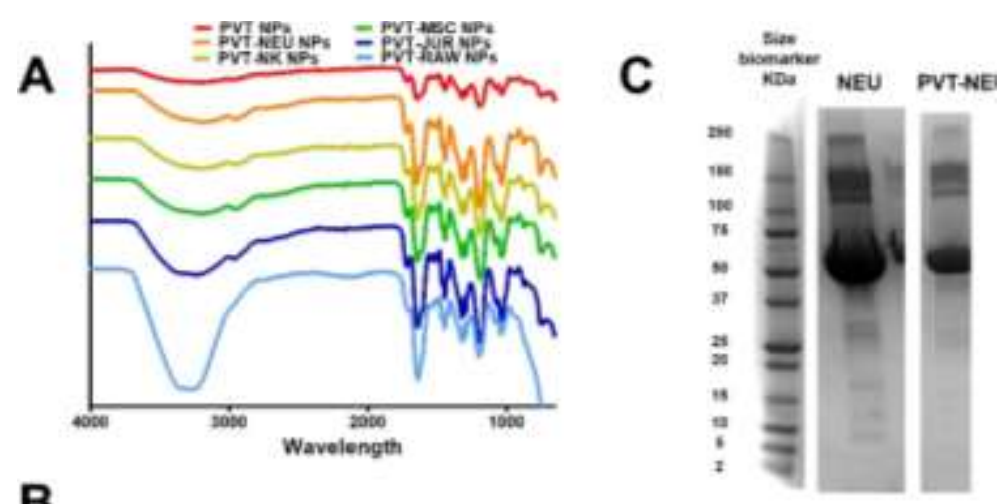

B

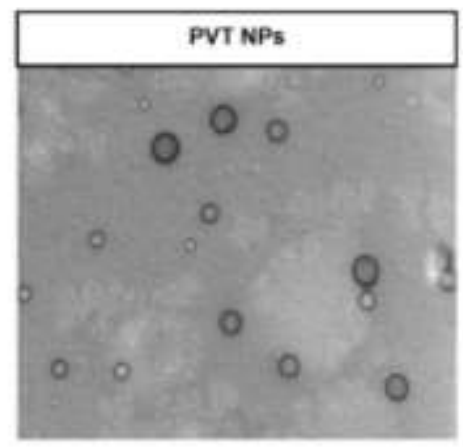

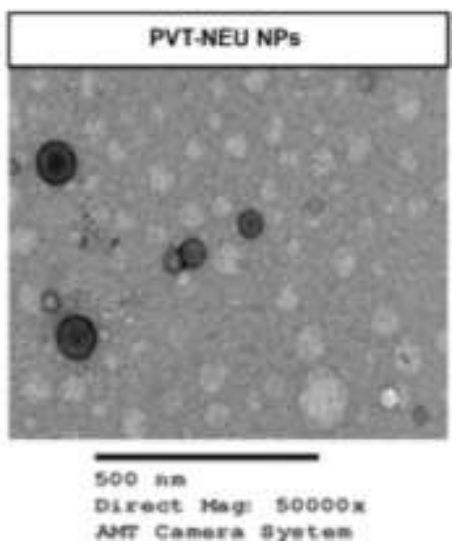

Figure 4-2. Evaluate the cloaking of cell extracted membrane on to the nanoparticles and the inclusion of PTX in the resultant PVT and PVT-MEM NPs

A) Fourier-transform Infrared (FTIR) spectral analysis of PVT and PVT-MEM NPs were acquired on a Universal Attenuated Total Reflectance (UATR) accessory plate by a Spectrum 100 FTIR spectrophotometer (Waltham, MA), between 4000 and $650 \mathrm{~cm}-1$ at a scanning speed of $4 \mathrm{~cm}-1$ for 32 scans. Characteristic N-H stretching at $3258 \mathrm{~cm}-1$ and $\mathrm{C}=\mathrm{O}$ stretching at 1715 cm-1 confirms presence of PTX consistently in PVT and PVT-MEM NPs. B) PVT and the representative PVT-MEM NPs transmission electron microscopic (TEM) images. UranyLess EM stain was used for negatively staining the nanoparticles. Image was acquired by using JEOL 200EX TEM at a direct magnification of $100,000 \times$. C) Presence of membrane proteins on NEU and PVT-NEU estimated by SDS-PAGE Coomassie blue staining. 
intensity with respect to control is shown in Figure 4-3A. A statistical significant difference between the coated PVT-MEM NPs in contrast to PVT NPs were seen in both the TNBC cells: MDAMB-231 and MDA-MB-468. MCF7 which was a non TNBC cell lines, shows no significant difference between coated and uncoated nanoparticles except PVT-RAW NPs. The highest intensity was observed for uptake of PVT-MEM NPs in contrast to PVT NPs in MDAMB-231 cells, whereas breast epithelial cells, MCF10A show very minimal internalization, demonstrating the preferential uptake in cancer cells versus non-cancer cells, (Figure 4-3B). In all the four tested cell lines, the fold change with PVT-NEU, PVT-RAW NPs is the highest in comparison to membrane uncoated PVT NPs, whereas PVT-MSC NPs displayed decent uptake in the TNBC cell lines. Thus, these three membrane coated nanoparticles (PVT-NEU. PVTMSC, PVT-RAW NPs) were selected for further studies. The Rest of the In Vitro, In Situ and In Vivo Studies from here onwards was conducted with PVT-MEM NPs (PVT-NEU, PVT-MSC and PVT-RAW NPs).

In situ cellular uptake [351] was utilized to determine preferential targeting by the PVTMEM NPs specifically to fixed tissue slides of various tumor grades. For this BC tissue array (TMA) with cores of normal adjacent BC tissue, Stage I which is ER, PR (-) ve. For fluorescent imaging (Figure 4-4A) C6 loaded particles were also used and quantified using Image J (Figure 4-4B). A targeted efficacy is observed for cancerous section versus normal adjacent tissue section, elaborating the preferential targeting ability of the PVT-MEM NPs, with significantly higher intensity observed for Type I which is aggressive and invasive ductal carcinoma, with PVT-NEU NPs showing the highest statistical increase in uptake with a fold change of 1.19 versus 1.02 and 0.92 for PVT-MSC and PVT-RAW NPs respectively, in comparison to uncoated PVT NPs. The in vitro cytotoxicity of PTX in solution and PTX loaded in the PVT and PVTMEM NPs were evaluated. Cell proliferation conducted by MTT assay (Figure 4-5A) with PTX concentration ranging from $0-20 \mathrm{nM}$ when treated on highly aggressive and metastatic MDAMB-231 and BC cells MCF7. The $\mathrm{IC}_{50}$ values were significantly lowered by 2.05 folds with PVT NPs in comparison to free PTX, whereas effects all the three selected PVT-MEM NPs showed similar profile, with PVT-NEU NPs demonstrating the most significant change of 2.95 folds reduction when compared to PTX alone, in MDA-MB-231. However, MCF7 demonstrates similar cell inhibitory effects with PTX, but no significant dose-dependent inhibitory effects were observed. Similar morphological changes at $5 \mathrm{nM}$ are displayed in Figure 4-6A proliferation panel, showing distinct morphological changes on treatment with PTX solution and PVT NPs at similar concentrations. To further confirm superior anticancer efficacy of PVTMEM NPs over free PTX, we conducted boyden migration assay, matrigel invasion assay and the colony forming ability on MDA-MB-231. As shown in Figure 4-6B through D. Migration/Invasion/Colony panels at $5 \mathrm{nM}$ equivalent PTX solution or nanoparticles treatment with PVT/PVT-MEM NPs, all show a statistically significant difference with the plain drug, PTX. Statistical significance was observed between PVT and PVT-MEM NPs, except in migration assay where there was no statistical significance observed between PVT and PVTNEU NPs, but a trend of inhibition was confirmed. These results altogether suggest the nanoparticles significantly improved therapeutic effect over the free drug. The 3D spheroid model of MDA-MB-231 was conducted to predict the inhibitory effects of PTX. An inhibition trend in tumor spheroid volume is observed in all the treatment groups (Figure 4-7A), where PTX loaded in PVT-MEM NPs resulted in shrinkage of spheroids, indicating concomitant loss of 3D structure of cells [352] demonstrating the anticancer potential of PTX remains intact in the 


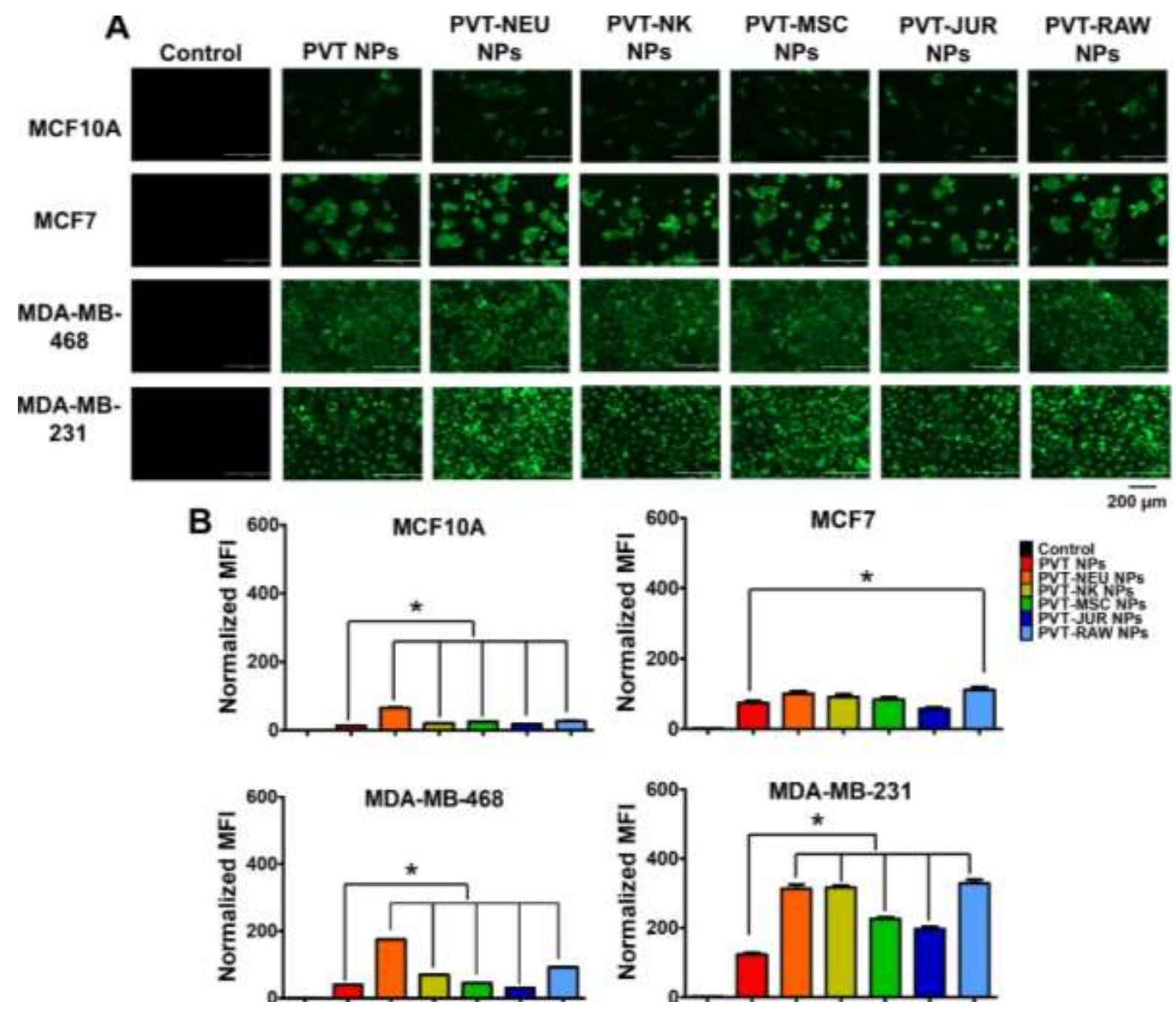

Figure 4-3. Intracellular uptake PVT and PVT-MEM NPs

A) Intracellular uptake of dye (C6) loaded nanoparticles, instead of drug, to view the fluorescent uptake of the nanoparticles, after allowing 3 hours of incubation. The excess dye adhered to the surface of the cells was washed with PBS, 3 times of 5 mins each. Cells were qualitatively imaged under the EVOS ${ }^{\circledR}$ FL Imaging System (AMF4300, Life Technologies, Carlsbad, CA, USA). B) Quantitatively analysis of the cellular uptake was conducted using Accuri C6 Flow Cytometer (Accuri Cytometer, Inc., Ann Arbor, MI, USA). FL1 channel (488 excitation, Blue laser, $530 \pm 15 \mathrm{~nm}, \mathrm{FITC} / \mathrm{GFP}$ ). Average reading and standard error of mean were calculated from 3 runs. 

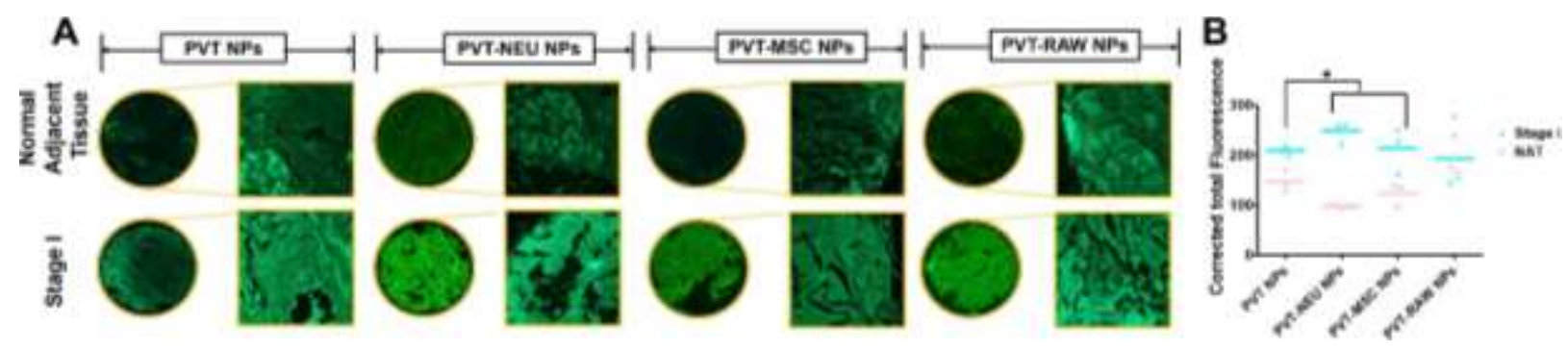

Figure 4-4. In situ dye uptake by PVT and PVT- MEM NPs

A) In Situ tumor targeting ability of PVT and PVT-MEM NPs were assessed using tissue microarrays (TMA) slides. Slides were incubated with C6 equivalent on each slide for overnight after processing them, followed by imaging. Type I represents the aggressive and invasive ductal carcinoma, shows highest fold change of 1.7 folds by PVT-NEU NPs versus 1.15 and 1.45 for PVT-MSC and PVT-RAW NPs respectively, when compared to PVT NPs. Image J was used to quantify the fluorescence intensity. Data were measured using the distance in pixel and pixel aspect ratio same.
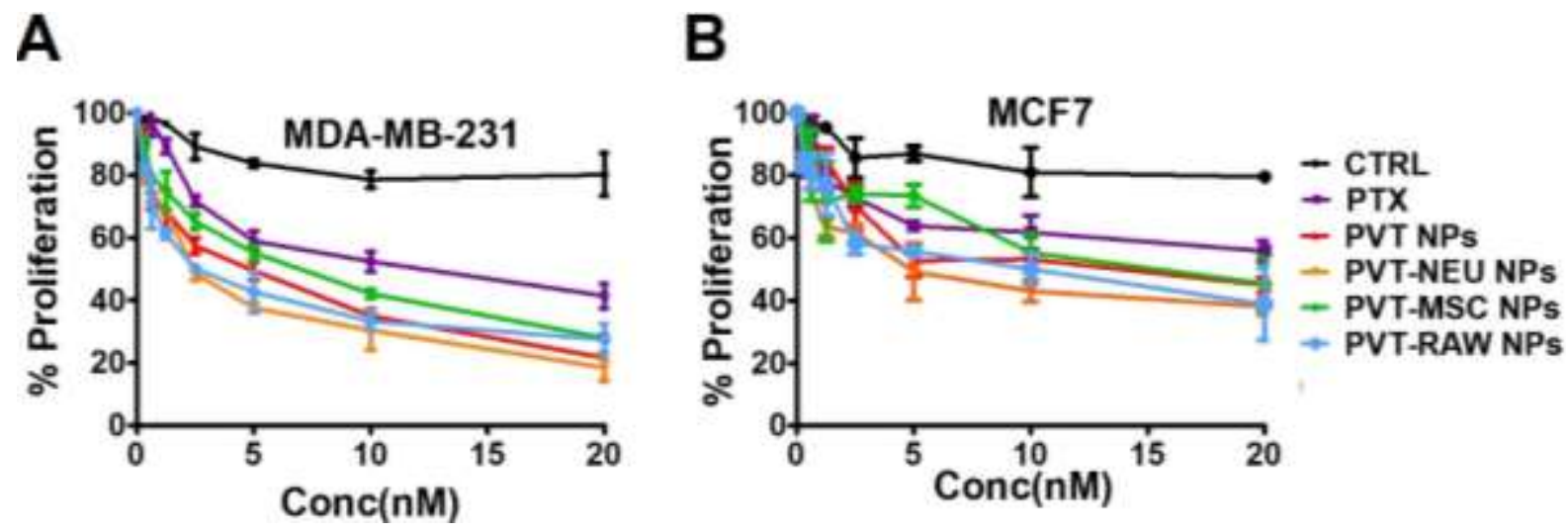

Figure 4-5. Cell proliferation assay by PVT and PVT-MEM NPs in TNBC cells A-B) Cell proliferation measured by MTT assay markedly decreased proliferation of TNBC cells (MDA-MB-231) in comparison to breast epithelial cells MCF7 that does not show concentration dependent proliferation, suggesting the specificity of the nanoparticles to TNBC cells after treatment with 0-20 nM (equivalent amount of drug) for 48 hours. Untreated cells were used as control. Cells treated with PVT and PVT-MEM NPs showed significant inhibition in cell proliferation when compared to PTX solution and absorbance was recorded by a Microplate Reader (BioTeK Cytation 3, Winooski, VT, USA) at $570 \mathrm{~nm}$. Data presented as mean + standard error of the mean $(n=3)$. 

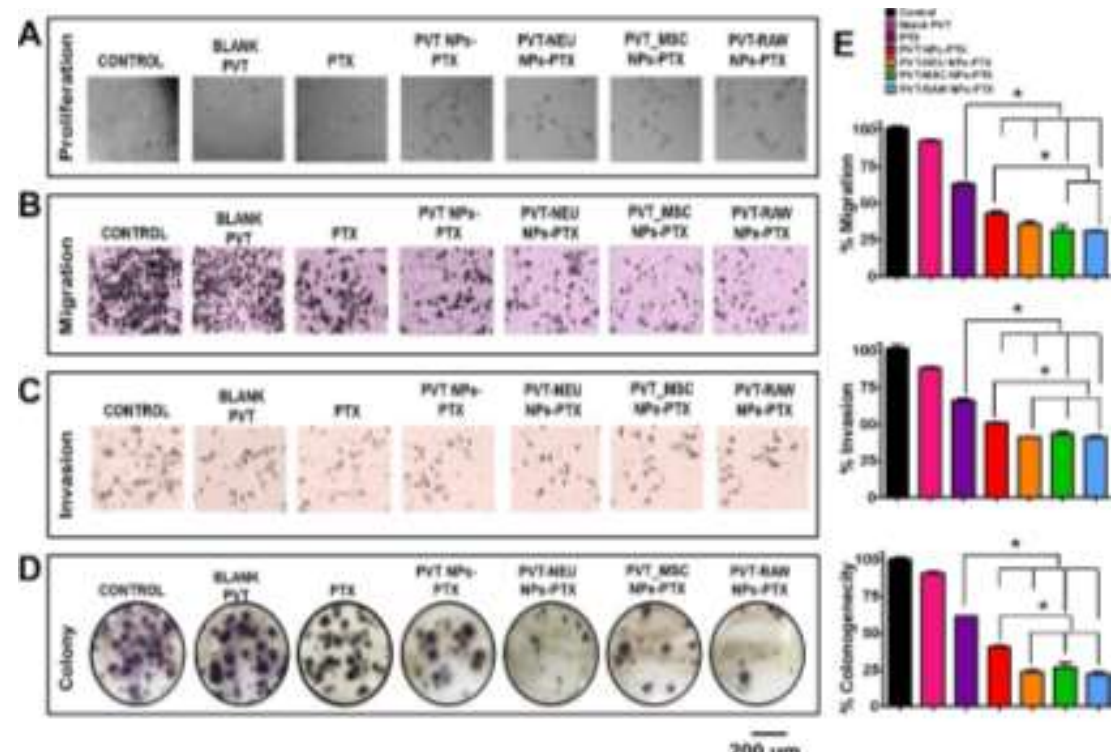

Figure 4-6. Anticancer efficacy of PVT and PVT-MEM NPs in TNBC cells

A) Panel Proliferation: shows bright field microscopy images after MTT analysis demonstrating significant morphological changes in MDA-MB-231 after 48 hours treatment. Imaging was done by an EVOS ${ }^{\circledR}$ FL Imaging System. B-D) Migration \& Invasion \& Colony: PVT-MEM NPs after exposure to $5 \mathrm{nM}$ equivalent of PTX shows higher inhibition in comparison to PVT NPs and significantly more than PTX solution at equivalent concentration. Significant changes in MDAMB-231 TNBC cells were noticed suggesting the targetability of PVT-MEM NPs. E) Quantification of percent migratory cells, percent invasive cells and percent clonogenicity, with respect to control cells. 


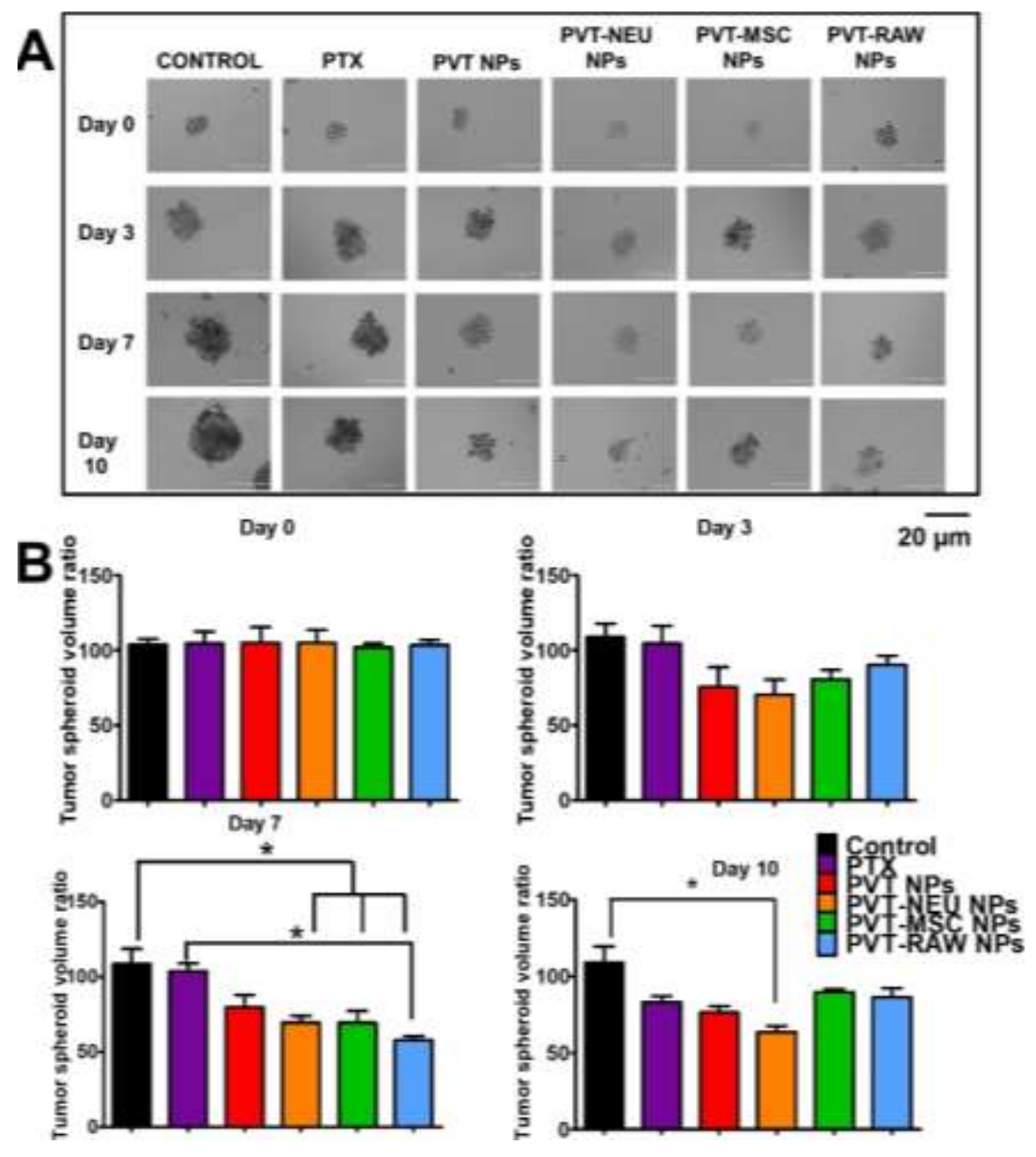

Figure 4-7. Spheroid forming ability of TNBC versus breast epithelial cells

A) Inhibition profile of MDA-MB-231 tumor spheroid growth was evaluated after treatment with PTX solution and PVT/ PVT-MEM NPs, respectively, PTX equivalent to $5 \mathrm{nM}$, untreated cells served as the blank control. Images were obtained at $0,3,7$ and 10 days under brightfield inverted microscope (EVOS ${ }^{\circledR}$ FL Imaging System). Image J was used to quantify the size of each spheroid, all measurements were done keeping distance in pixel and pixel aspect ratio same. (B) Tumor spheroid volumes ratio (\%) was calculated based on change in size each day with respect to day 0. Data presented as mean + standard error of the mean $(n=3)$. 
in the PVT and PVT-MEM NPs. As seen in Figure 4-7B there was no inhibition observed at day 0 . As time elapsed, the inhibition trend was pronounced, with statistical difference observed in PVT-MEM NPs with respect to control, on day 7 and day 10. A statistical significant inhibition was noticed between PVT and PVT-RAW NPs on day 7. Although, there was no statistical significant difference observed between PVT and PVT-MEM NPs, yet an inhibition trend could be confirmed, exhibiting the intact inhibitory effect of PVT-MEM NPs after the membrane coating.

\section{In Vivo Imaging, Biodistribution, Tumor Retention and Efficacy Studies}

The fluorescently labeled PVT and PVT-MEM NPs loaded with ICG were used to evaluate the in vivo distribution in orthotopic xenograft tumor-bearing mice. MDA-MB-231 cells were used for this and the mice were subjected to treatment with the ICG dye loaded nanoparticles after the tumors reached $\sim 200 \mathrm{~mm}^{3}$. Mice treated with ICG solution were used as a control for this study and tumor retention and biodistribution of the dye in mice when delivered (after a single dose) by PVT or PVT-MEM NPs were evaluated. ICG dye equivalent to 200 $\mu \mathrm{g} / \mathrm{ml}$ were injected and mice were subjected to imaging at regular intervals from 0-120 hours (Data shown for 72 hours). As shown in Figure 4-8A ICG solution is cleared out rapidly from the system than any of the other treated groups, suggesting the prolonged retention and longer circulation due to the enhanced EPR of nanoparticles that makes PVT and PVT-MEM NPs retain longer. However, a conventional nanoparticular system such as PVT NPs though was retained longer than ICG solution, they are cleared faster than the PVT-MEM NPs. PVT-MEM NPs due to their surface coating showed a slower clearance rate as seen from their longer circulation at least till 48 hours for all the groups, as shown in Figure 4-8A (Panels: PVT-NEU, PVT-MSC, PVT-RAW NPs). As shown in Figure 4-8B the fluorescence intensities of PVT-MEM NPs were greater than ICG and also for PVT NPs, that did not have long circulation time in comparison to all the PVT-MEM NPs. PVT-NEU demonstrated statistically significant fold change of 9.13, 11.22, 14.52, 15.90 and 10.52 folds for 3, 6, 24, 48 and 72 hours with respect to ICG treated mice (which were experimental control) when compared to PVT NPs. PVT-RAW NPs showed the least difference with only less than a fold increase with respect to ICG for 72 hours.

After 120 hours of injecting the dye loaded nanoparticles and imaging the mice at regular intervals, mice were euthanized and their orthotopic tumors and other organs were excised and imaged for fluorescence intensity. The accumulation of nanoparticles on the other organs, such as heart, lungs, liver, kidney, and spleen, are shown in Figure 4-9A. As shown, little or no retention of ICG was found indicating the rapid clearance of the dye due to the absence of a delivery system way before 120 hours. Tumor retention of ICG dye after 120 hours was considerably higher with 2.64 folds increased retention observed in PVT-NEU NPs, when compared to PVT NPs. Although there was no statistical difference in tumor retention observed with PVT versus any of the PVT-MEM NPs, yet the biodistribution study in the other organ provides insights about the benefit of using the membrane coating. An ex vivo analysis (Figure 4-9B) of PVT and PVT-MEM NPs shows the highest retention in the liver in comparison to any other organ. There was significantly lower retention of the dye in all the organs, except the liver (heart, lung, kidney, spleen). Although there was no statistical difference of the dye in the liver through PVT and PVT-NEU NPs, unlike all other organs, yet a trend of lower retention is seen. 


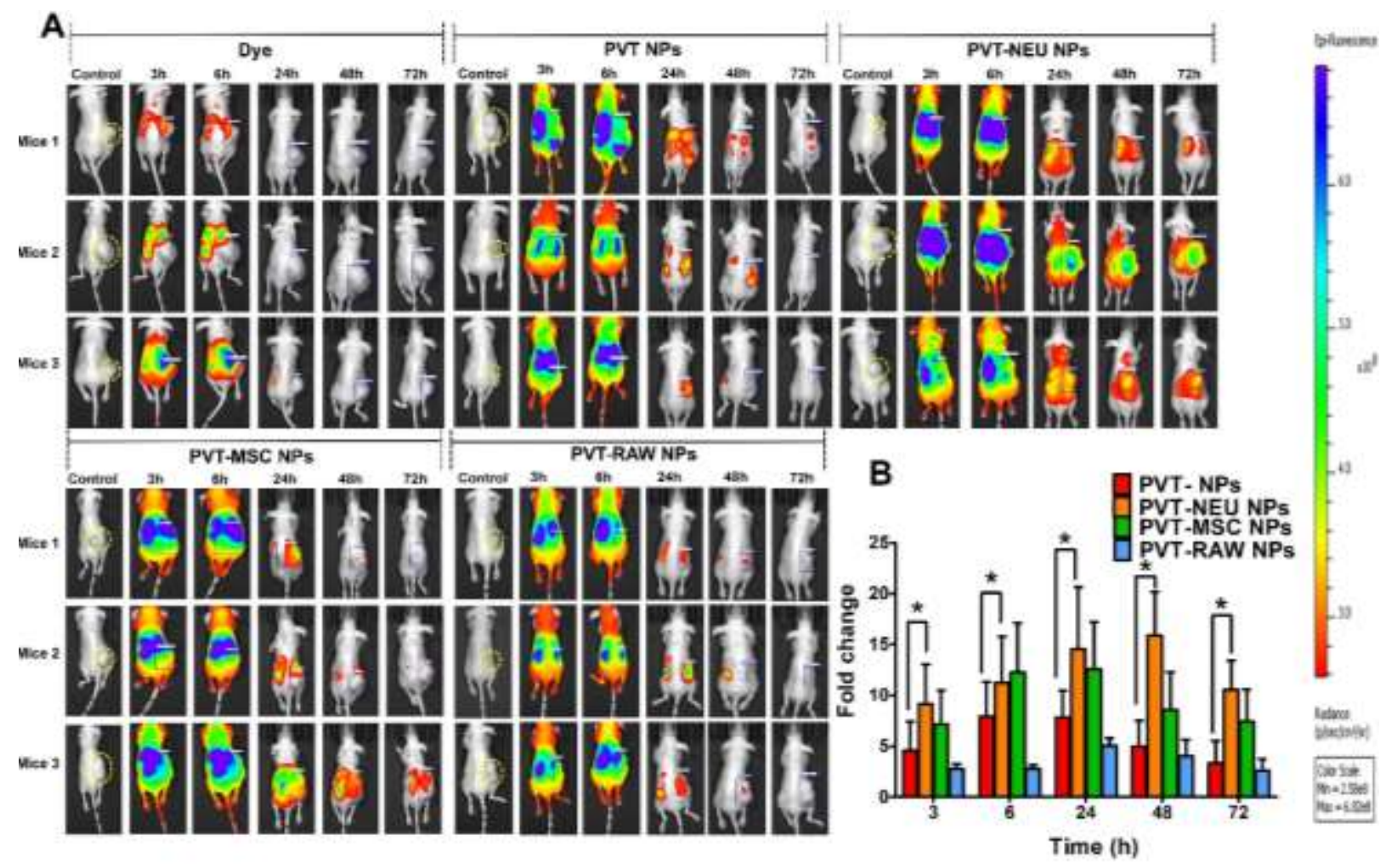

Figure 4-8. In vivo tumor immunofluorescence retention of PVT and PVT-MEM NPs loaded with Indocyanine green

A) Immunofluorescence imaging in a time dependent manner was assessed using Indocyanine green loaded PVT and PVT-MEM NPs. After palpable tumors of MDA-MB-231 cells were grown, mice were treated with ICG equivalent of $100 \mu \mathrm{g} / \mathrm{ml}$ of PVT or PVT-MEM NPs, plain ICG solution was used as experimental control. Mice were subjected to imaging after 3, 6, 24, 48 and 72 hours to determine the retention of the fluorescent dye using IVIS Spectrum system (Caliper Life Sciences). ICG was cleared out within 24 hours, whereas PVT-MEM NPs prolonged retention over PVT NPs. B) To assesses the fold change of immunofluorescence intensity signifying prolonged retention and distribution of membrane coated nanoparticles over uncoated nanoparticles in of PVT-MEM NPs when compared to PVT NPs were calculated. Data calculated from ROI mean values + standard error of the mean $(n=3)$. 
A ICG
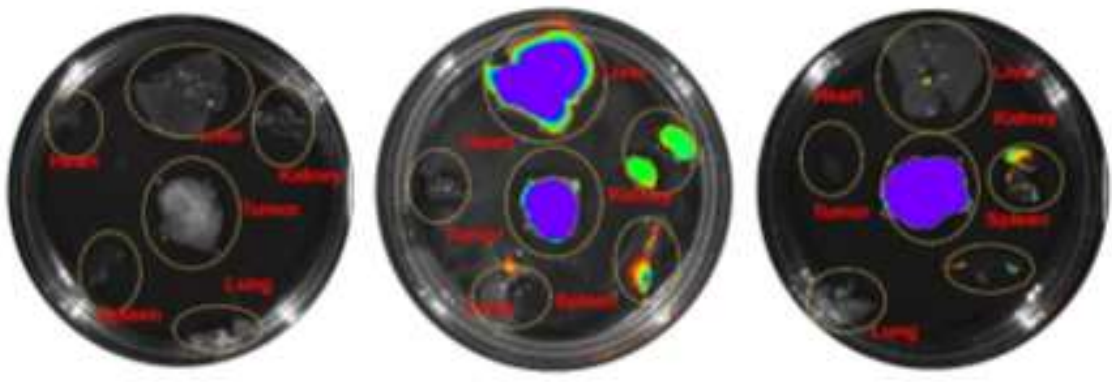

PVT-MSC NPs

PVT-RAW NPS

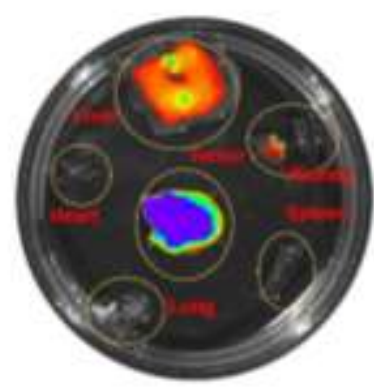

B

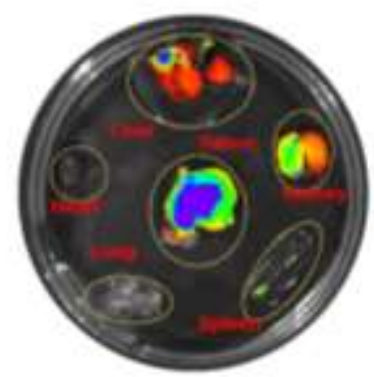

Liver retention
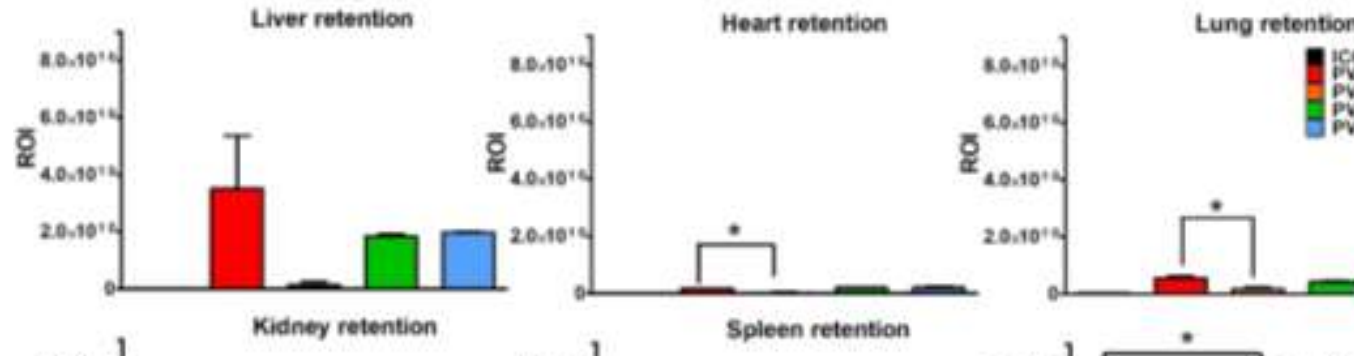

Spleen retention
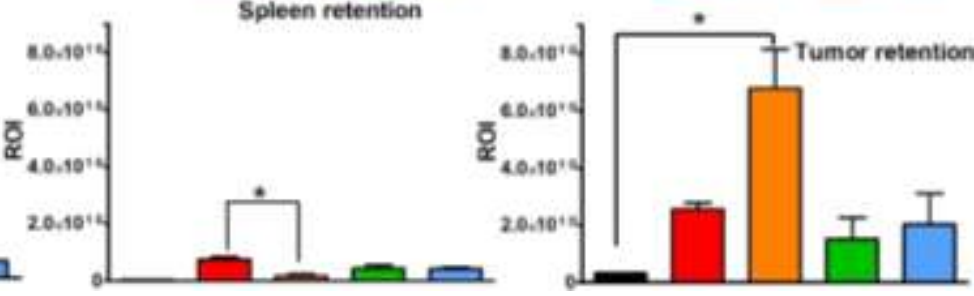

Figure 4-9. Ex vivo immunofluorescence of excised tumors and organs

A) Ex Vivo biodistribution of immunofluorescent dye ICG after 120 hours of intraperitonially injection of ICG loaded PVT and PVT-MEM NPs. B) Quantitative analysis of the fluorescence intensity of excised organs, heart, lungs, liver, kidney, spleen and tumor were collected, and ROI value collected and assessed using IVIS Spectrum system (Caliper Life Sciences) at the UTHSC core facility. 
Altogether, based on less biodistribution to other organs, prolonged longer circulation, and higher tumor retention PVT-NEU was selected for evaluating therapeutic efficacy in in vivo xenograft model. The ability of PVT and PVT-MEM NPs in combination with PTX to inhibit primary tumors was evaluated in MDA-MB-231 tumor-bearing xenograft mouse model. After the tumors reached a palpable size $\sim 200 \mathrm{~mm}^{3}$, they were randomly separated into 6 groups (5 mice each), before initiating treatment with $10 \mathrm{mg} / \mathrm{kg}$ PTX or PTX equivalent in PVT/ PVTNEU NPs, given intraperitoneally, twice a week. Control mice received PBS treatment. PTX solution showed $\sim 52 \%$ tumor inhibition in comparison to control mice (Figure 4-10A through D), whereas $67 \%$ and $72 \%$ inhibition was exhibited by PVT NPs PVT-NEU, in comparison to control group.

A statistically significant difference was observed in inhibition profile of PTX versus PVT and PVT-NEU NPs, however there was no statistically significant difference observed between the PVT and PVT-NEU NPS, yet the inhibition trend was confirmed. To assess the biocompatibility and hemotoxicity, fresh blood was withdrawn by cardiac puncture. As indicated in Figure 4-10E mice group treated with PTX show systemic toxicity, exhibited from the distorted morphology of the RBC, whereas PVT NPs and PVT-NEU NPs exhibit good biocompatibility, probably due to the goodness of nanotechnology. To analyze if PTX induces any adverse toxic effects, we evaluated the serum level of liver enzymes (ALT, AST, TBI), kidney function markers (BUN) (Figure 4-11A). ALT 17-77 U/L, AST 54-298 U/L, TBili 0.0$0.9 \mathrm{mg} / \mathrm{dL}$ and BUN 8-33 mg/dL were considered normal ranges. Although there was slight elevation of AST and TBili in PVT and PVT-MEM NPs, signifying the potential liver damage caused probably by PTX $[353,354]$, also seen the liver retention in the biodistribution studies. Other than that, all the other tested enzymes were normal, exhibiting regular kidney function. On the contrary, PTX significantly reduced the number of WBC, neutrophil and lymphocytes with respect to control, as seen in Figure 4-11B. Conversely, no myelosuppression was noticed in PVT-NEU NPs with counts similar to control, although a slight decrease of WBC, neutrophil and lymphocytes in PVT NPs with respect to control. Although there was no statistically significant difference between PTX and PVT or PVT-NEU NPs, nevertheless, there a trend in increase in neutrophil count by $43.33 \%$ in contrast to PTX was observed, lesser chances of PVT-NEU NPs to cause neutropenia unlike PTX solution [355].

Tumors were excised and collected for IHC and H\&E histological analysis, as shown in Figure 4-12. As shown from the images, there was an increase in apoptotic markers, BAK and Cleaved Caspase-3, and decrease in cell survival protein Bcl-2 expression in tumors treated with PVT-NEU comparatively more than PVT NPs and PTX solution. The control shows upregulation of PCNA, a cell proliferation marker whereas with treatment groups there is a decrease in PCNA expression. IHC analysis for $\beta$-tubulin shows a higher expression of protein in tumor treatment with treatment groups as compared to control indicating the treatment causes mitotic cell cycle arrest of the tumor cells. The $\beta$-tubulin expression is enhanced with PVT-NEU NPs over PTX, suggesting more inhibition of microtubules causing cell cycle arrest during mitosis [327]. Panel H \& E staining shows apoptosis, evident from the clusters of the nuclei that are consistent with tumor inhibition, where PBS treated control group displayed fewer apoptosis seen from the intact nuclei. Taken together, although there was no significant therapeutic efficacy observed between PVT and PVT-NEU NPs, yet PVT-NEU NPs demonstrated a comparatively safer profile in terms of biocompatibility, biodistribution to healthy organs and 
A
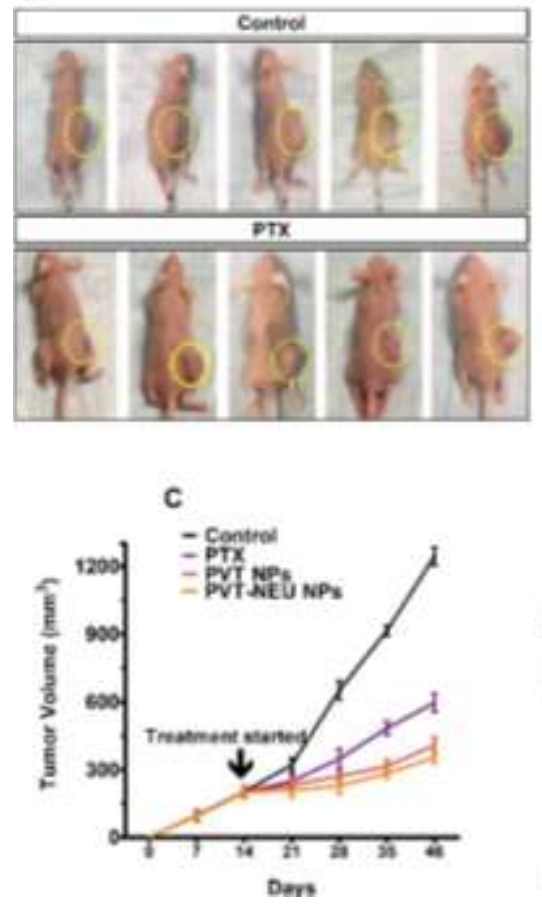
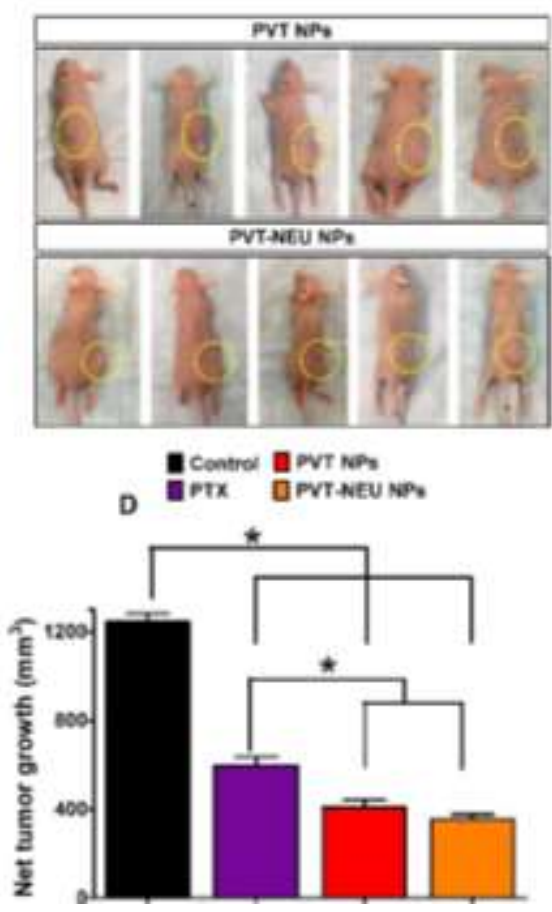

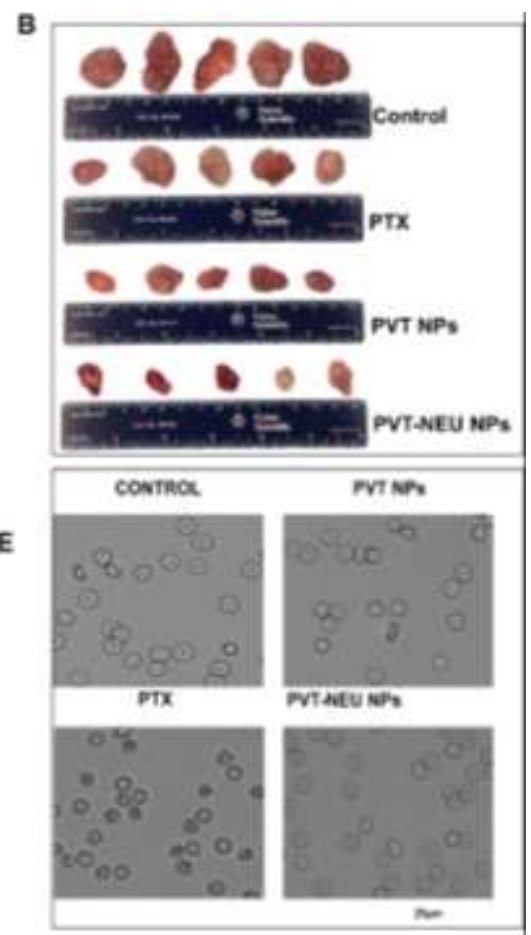

Figure 4-10. Tumor growth inhibition profile of PVT and PVT-MEM NPs (PVT-NEU, PVT-MSC, PVT-RAW NPs)

A-B) PVT-NEU and PVT-RAW NPs shows significant inhibition with respect to PTX solution, where PTX equivalent was $(10 \mathrm{mg} / \mathrm{kg})$, in MDA-MB-231 xenograft mice model, as seen from the whole mice images just before the tumors were excised and excised tumors. C) Average tumor volume of control and PTX, PVT and PVT-MEM NPs respectively after 4 weeks treatment. D) Net tumor growth of MDA-MB-231 tumors in control and PTX versus PVT-MEM NPs group. E) Hemocompatibility was assessed from red blood cell morphological changes, after blood was collected at the end point of the study by cardiac puncture. Data in bar graph represent mean \pm SEM of six tumors in each group. Data presented as mean + standard error of the mean $(\mathrm{n}=5$. 

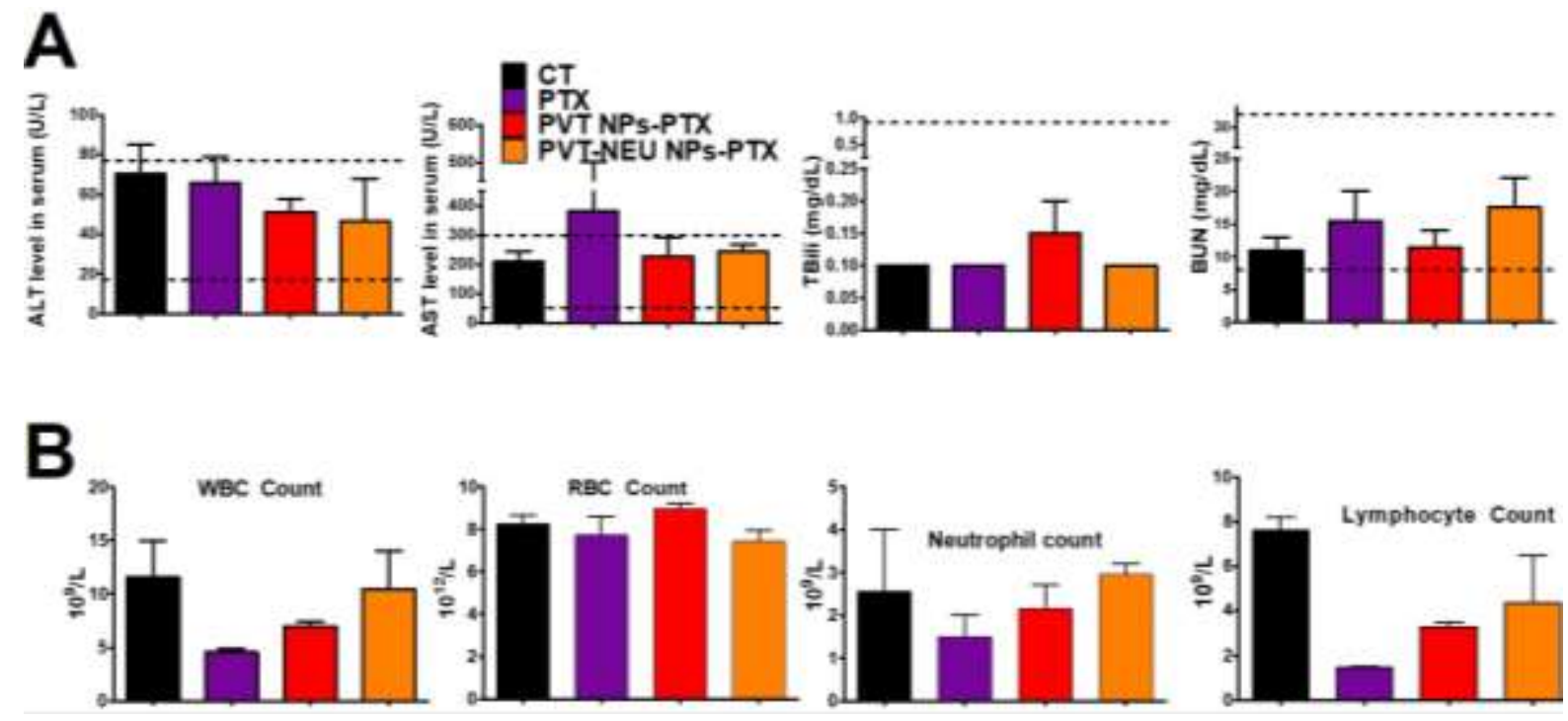

Figure 4-11. Evaluation of potential toxicity of PVT and PVT-MEM NPs PVT-NEU, PVT-MSC, PVT-RAW NPs)

A) From Left to Right: Serum chemistry levels of liver enzymes (ALT, AST, TBI) and kidney function markers $(\mathrm{BUN})$ were determined in all the groups, dotted black line indicates the normal range for each of the enzyme. B) From Left to Right: Blood count analysis determined levels of WBC, RBC, neutrophil and lymphocytes were determined. WBC, Neutrophil and lymphocyte count was significantly lower in PTX treated group, indicating changes of myelosuppression, in contrast to PVT-NEU NPs that has WBC, RBC, neutrophil and lymphocyte equivalent to control group. Data presented as mean + standard error of the mean ( $\mathrm{n}$ $=5)$. 

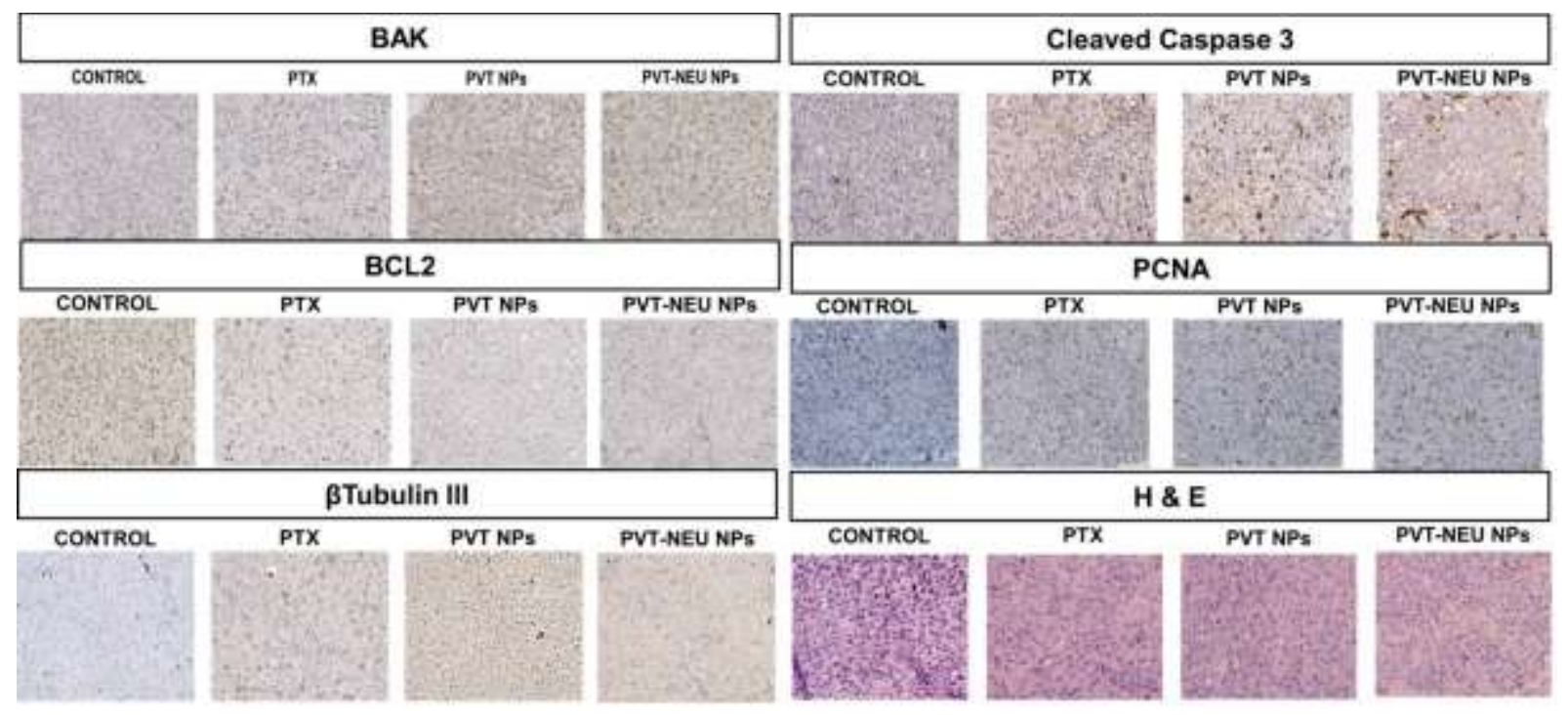

$50 \mathrm{~nm}$

Figure 4-12. Immunohistochemistry analysis and histological evaluation of tumors treated with PVT and PVT-MEM NPs PVT-NEU NPs

A) Representative immunohistochemical images of excised xenograft tumors for expression of apoptotic markers, BAK and Cleaved Caspase- 3 and cell survival protein Bcl-2, proliferation marker PCNA, $\beta$-tubulin suggesting mitotic cell cycle arrest and H\&E staining for distinguishing morphological difference. 
analysis of various enzymes and blood work. These demonstrate the potential of biomimetic PVT-MEM NPs especially PVT-NEU NPs due to good biocompatibility and other effective therapeutic virtues could possibly make progress as a personalized tool for cancer therapeutics.

\section{Discussion}

One of the main causes of hindrance in the development of treatment modalities for TNBC, is the aggressive and metastatic nature of this subtype of BC. It is characterized by lack of expression of estrogen receptor (ER), progesterone receptor (PR) and human epidermal growth factor receptor (HER2) [362]. This type of malignant related disease must have proper detection, treatment, and prevention from metastasis. However, lack of finding a prognostic marker, the heterogeneity, and the aggressiveness, makes treatment to this subtype of BC a formidable challenge. Currently, the mainstay treatment option even for this of metastatic disease is primarily systemic administration of chemotherapeutics, administered as a single agent or in combination with immunotherapy. These agents though have potential therapeutic benefits to the primary tumors yet pose adverse effects on non-targeted healthy tissues with low penetration of drugs at the metastatic region. Therefore, the development of novel drug delivery strategies that can improve the selectivity of drugs to the primary tumors with localization at the metastatic regions and minimize toxicities to normal tissues, is crucial. [122]. Based on molecular profiling, several targets for TNBC have been established [363], which has made it possible for several potential compounds/drugs to make it to the clinical trials, to be evaluated in TNBC patients [364]. Although several efforts are made by scientist across the globe, there are only a few FDA approved targeted therapy that has made it through the clinical trial. Among them, very recently Lynparza (olaparib), a PARP inhibitor was approved as monotherapy for BRCA1/2-mutation TNBC patients [365]. Additionally, immunotherapy drug Tecentriq (atezolizumab), given in combination with the chemotherapeutic drug Abraxane, for PD-L1-positive TNBC received accelerated approval for treatment of TNBC, where PD-L1 comprises about $\geq 25 \%$ of TNBC patients [366].

Novel techniques such as active targeting by attaching ligands (such as small molecules, peptides, aptamers, and antibodies) on nanoparticles to specifically recognize and bind to receptors on target cancer cells for enhancing targetability drug molecules in tumors. Such strategies would also efficiently minimize off-targeting problems, maximize the therapeutic window and would ensure maximum survival rate and improve the quality of the patient's life. Among the many new strategies employed for targeted therapy, one such strategy is the functionalization of particles with biological compartments or naturally derived cell membranes [356]. Biomimetic nanoparticles combine the characteristics of nanoparticles with cell membrane characteristics, imparting a biological identity to these nanoparticles [357]. Thus, providing enhanced targeting by interaction with the complex biological microenvironment imparting, specificity, improved circulation, non-immunogenicity and potent therapeutic response [341]. The longer the nanoparticles are in circulation, ensures controlled delivery of the drug entrapped in the nanoparticles and being capable of reversible binding to entrapped target proteins. This creates a dynamic compartment for specific vascular distribution of drugs. They target selected disease sites, thus protecting the surrounding healthy cell from toxicity. These engineered nanoparticles are modified in such a way that they are non-immunogenic to the body, thus ae not 
opsonized, or being recognized by macrophages and rapidly cleared from circulation $[67,68]$. Various cell membranes have been explored to camouflage nanoparticles with red and white blood cells, platelets, viruses, exosomes, extending potential therapeutic response and mimicking properties (Figure 4-13) [358-362]. Based on these reports and our preliminary findings, we are investigating alternative strategies that could be used for functionalizing the biomimetic nanoparticles, that would provide a specific binding to tumors as well as metastatic sites, such that systemic administration of chemotherapeutic drugs can be minimized. We initially developed a PVP and tannic acid-based nanoparticle, both have their associated advantages. It was reported by Le et. al. that PTX molecules aggregates to form the hydrophobic core with tannic acid by a hydrogen bond. However, on addition of PVP to this tannic acid-PTX core compact and stable core could be achieved, due to stronger hydrogen bond interaction [363]. We thought, this would be an ideal stabilized core for our membrane coated nanoparticles, as the membrane coating on the top of the nanoparticles would be efficient with a rigid core. This is the reason we choose PVT (Polyvinylpyrrolidone-Tannic acid self-assembled) nanoparticles. At first we developed a nanoparticle core (PVT NP) based on our previous findings [300, 344], that shows therapeutic efficacy over commonly administered chemotherapeutic agent, PTX in TNBC and prevents $\mathrm{P}$-glycoprotein mediated drug efflux, one of the contributing factors for reduced efficacy of PTX in TNBC patients [364-366].

PVT NPs were coated with neutrophil membrane (NEU) because when activated, shows presence of membrane proteins on the bilayer, such as, Lymphocyte function-associated antigen1 (LFA-1), macrophage-1 antigen (Mac-1), platelet endothelial cell adhesion molecule-1 (PECAM-1), P-selectin glycoprotein ligand-1 (PSGL-1) that have high affinity for receptor site specific on TNBC [367]. The membrane proteins from the SDS-PAGE Coomassie stain show presence of LFA-1, MAC-1, PSGL-1 and PECAM-1 (molecular weights: 140KDa, 47KDa, $\sim 43 \mathrm{KDa}$ and $\sim 130 \mathrm{KDa}$, respectively). The profile of the membrane proteins on both NEU and PVT-NEU seems to be intact around those molecular weights. Although, the presence of the proteins in PVT-NEU seems to be a little faint than NEU, but the presence of the membrane proteins suggests proper translocation of these proteins on the surface of PVT nanoparticle. We would suggest a further verification by western blotting and bottom up proteomics analysis [368]. This explains the enhanced PVT-NEU NPs accumulation at the tumor site, due to the overexpression of ICAM-1, VCAM-1 and E-selectin on TNBC that alters tight junctions between endothelial cells, resulting in increased vessel permeability [357, 369-371].

Natural killer (NK) cells are known to recognize cancer cells and have a cytotoxic effect on them [372, 373], due to increased expression of markers such as killer-cell immunoglobulinlike receptors (KIRs) and natural cytotoxicity receptors (NCRs) [372, 374]. However, in tumor microenvironment due to the secretion of soluble factors by the tumors, the NK cell activity may be impaired, resulting in escape of NK mediated killing [375, 376]. Taking advantage of this situation, we selected NK cells due to the presence of the activating markers that have shown to be beneficial in TNBC patients [373,377], which could be used to coat the nanoparticles. Mesenchymal stem cells (MSC) has shown to have an antiproliferative role on TNBC cells especially MDA-MB-231, as seen from in vitro proliferation, migration and angiogenesis assays, suggesting the potential of using MSC for treatment of TNBC [378]. Leukocyte membranes, when coated over nanoporous silica particles, has expression of LFA-1 that has specificity for ICAM-1 on TNBC cells [379]. Based on these findings, we selected Jurkat cell membrane, 


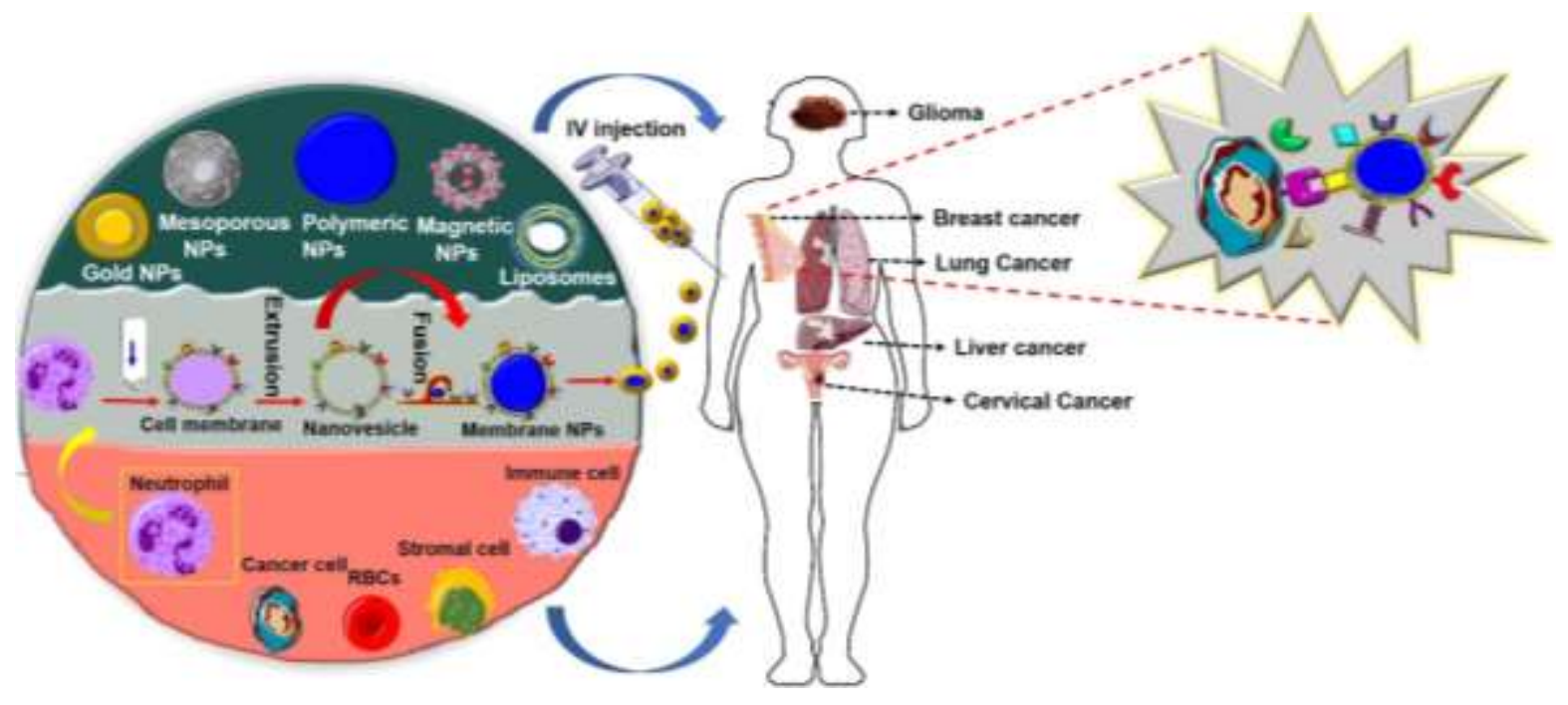

Figure 4-13. Schematic representation of the preparation of cell membrane-camouflaged nanoparticles

Various source cells used to extract cell membrane vesicles and coated on the top of the nanoparticles designed for a variety of cancer therapy, having selectivity to the membrane surface proteins. 
hoping that the isolated membrane would preserve some of the biological activity and would cause enhanced adhesion to the tumor vasculature [362]. Macrophages cells or RAW 264.7 show high expression of $\alpha 4$ and $\beta 1$ integrins, that can bind to the metastatic BC cells due to the cellspecific binding with vascular cell adhesion molecule-1 (VCAM-1) [380].

We choose neutrophil, natural killer, mesenchymal stem cells, Jurkat cells and RAW 264.7 cells and extract the cell membrane for cell-specific binding of nanoparticles to the sitespecific tumor site and metastatic region in TNBC. After extraction of cell membranes, we coated the nanoparticles with these membranes, which is a lipid bilayer, which probably explains the slight increase in particle size and TEM images of PVT-MEM NPs over PVT-NPs. Also, the hydrophobic interaction was due to the electrostatic repulsion between the negatively charged PVT cores and the lipid membrane, that forms the coating over the nanoparticles, which otherwise would have formed aggregates [381]. The binding of the generated nanoparticles with the TNBC cells was confirmed using the intrinsic protein fluorescence quenching especially the tryptophan residue, that responds to binding or any chances in the local environment surrounding the indole ring [382]. To evaluate the interaction of activated neutrophil membranes (NEU), membranes from NK, MSC, JUR and RAW coated nanoparticles with aggressive and metastatic TNBC cells (MDA-MB-231) was used. Our intracellular and in-situ uptake shows the higher uptake of PVT-MEM NPs, as per compared to PVT NPs when the nanoparticles were loaded with fluorescent dye C6. In Vitro cytotoxicity assays as seen from cell proliferation, migration, invasion, and colony-forming assay, suggest the efficient anticancer ability of PVT-MEM over PVT NPs in TNBC cells. To further asses our results, we investigated the therapeutic efficacies on 3D spheroid cell culture matrix which is better predicator of drug resistance and efficacy due to the presence of oxygen and nutrient resulting in invasive cells on the outside and quiescent or necrotic cells inside of the spheroid [383]. These studies suggest, the in vitro activity of the nanoparticles were intact upon the membrane coating and did not have any impact on the anticancer potential, A plausible reason would be a presence of self-markers on the surface of the PVT-MEM NPs that provides cell-specific binding to the potential targets on TNBC cells, that explains the enhanced uptake on MDA-MB-231 versus other cell lines especially the breast epithelial cells, MCF10A.

In Vivo circulation time of plain ICG shows clearance of the dye completely within 5 days probably because of the clearance by RES, also expected to produce subtherapeutic tumor concentration [384] over nanoparticles that though prolonged circulation over plain solution due to the EPR effect yet are not circulated long enough as PVT-MEM NPs, that prolongs circulation of membrane coated nanoparticles, seen throughout all the time points observed in the bioimaging study. However, ex vivo analysis shows that higher accumulation of dye by PVT and PVT-MEM NPs occurs in the liver which is probably because it is a highly perfused organ and is known to cause unescapable monocytic macrophage uptake [385]. Orthotopic xenograft mouse model for TNBC was established using MDA-MB-231 to generate spontaneous metastasis model of BC through subcutaneous or orthotopic injections, and are considered suitable model for evaluation of therapeutics and anticancer drug candidates for $\mathrm{BC}[352,386,387]$. The accumulation of membrane coated nanoparticles over plain nanoparticles causes higher tumor inhibition as seen from PVT-NEU in in vivo model. After evaluation of the therapeutic potential of PVT-NEU NPs and its improved efficacy over PTX solution, the safety perspective was evaluated from serum enzymes and blood count. PTX is known to cause leukopenia and 
neutropenia and severe peripheral neurotoxicity which often ranges from $30 \%$ to $40 \%$ of patients receiving PTX chemotherapy [331]. Although no statistical significance was observed yet a lower trend to cause myelosuppression or neutropenia could be suggested for PVT-NEU over PTX solution. Additionally, no hemotoxicities was observed to regular red blood cells except for

PTX solution, which also correlates to the toxicities observed from serum enzymes. Furthermore, IHC analysis shows enhanced apoptosis wit PVT-NEU over other treatment groups morphology of the distorted tumor elucidates the enhanced therapeutic activity. Taken together, we demonstrate the cell membrane coated nanoparticles could be utilized for efficient delivery of anticancer drugs to the TNBC cells, showing improved therapeutic effects.

\section{Conclusion and Future Direction}

In this study, we have demonstrated that the cell membrane-based nanoparticular system provides prolonged circulation and enhanced therapeutic efficacy in TNBC cells, MDA-MB-231 for targeted delivery of the potential therapeutic agent, PTX. This is due to the overexpression of adhesion moieties and/or selectins on TNBC cells that serves as a receptor-ligand for interaction with the activated protein moieties on the surface of the membrane coated nanoparticles. Although, further confirmation about the presence and abundance of the membrane-associated proteins on the surface of the nanoparticle, after extrusion and coating can be confirmed through bottom-up proteomics analysis. This would provide estimation for the quantitative analysis of nanoparticles that would retain the membrane associated proteins from the source cells and are being translocated on the top of the nanoparticles to provide selectivity. Herein, we report that neutrophil membrane coated nanoparticles due to the cellular expression, potentiates binding to the primary tumor and attenuates adverse toxic effects extended by the potent yet cytotoxic drug PTX. 


\section{CHAPTER 5. CONCLUSIONS AND FUTURE PERSPECTIVES}

Nanotechnology-based drug delivery systems have been utilized to overcome the limitations of conventional drugs. Nanoparticle-based drug delivery approaches offer penetration capability at the diseased regions, prolong circulation time, improve toxicity and tolerability. With this respect, Abraxane, was able to reduce toxicity issues of Taxol ${ }^{\circledR}$ by improving the overall response rate in patients by $\sim 2$-fold improvement in contrast to PTX solution or Taxol ${ }^{\circledR}$. Despite the immense advances there are few unmet needs that suffers adverse effects, such as neutropenia, neuropathy. PTX is formulated as suffers serious side effect of sensory axonopathy and ganglion-neuropathy, neutropenia, severe peripheral neurotoxicity which often ranges from $30 \%$ to $40 \%$ of patients receiving chemotherapy. However, incidence of both neuropathy and neutropenia was significantly higher than native PTX drug. Thus, a dire need to mitigate this deadly disease TNBC, due to the aggressive and metastatic nature is required. The main objective of this research is to develop a delivery system that can provide active targeting at the primary tumor site but can penetrate the of dense extracellular matrix and have therapeutic concentration for tumor penetration. Also, specificity and targetability would ensure the adverse side effects to other organs is minimized. This was first achieved by fabrication of polymer based, polyvinylpyrrolidone (PVP) PTX self-assembled nanoparticles with improved anticancer efficacies (Chapter 2). In further development of a nanoparticle system that would possess builtin chemosensitization motifs, a polyphenolic compound tannic acid-based PTX nanoparticles was fabricated that prevent drug efflux from cancer cells (Chapter 3). Finally, the combined effect of both these nanoparticles to obtain the core for biomimetic nanoparticle, that forms a rigid core for the nanoparticle on which membranes from endogeneous cells were coated to attain cell specificity to TNBC cells, as demonstrated in in vitro and in vivo models (Chapter 4).

The following summary can be drawn from Chapter 2:

- Screening of commonly used polymer and pharmaceutical excipient, for development of self-assemblies of PTX (polyvinylpyrrolidone, PVP).

- The formed self-assemblies exhibited greater cellular interaction and internalization in $\mathrm{BC}$ cells.

- The self-assemblies induce superior antiproliferation activity and cellular apoptosis in BC cells.

- This self-assembly nanoplatform laid the foundation for further development of a drug delivery system that can specifically target TNBC cells.

The following summary can be drawn from Chapter 3:

- Development of tannic acid-based PTX nanoparticles, that is known to exhibit anticancer and chemosensitization properties.

- Nanoparticles exhibited enhanced intracellular drug uptake in TNBC in comparison to PTX drug solution. 
- P-gp drug efflux was inhibited by these nanoparticles over drug solution, suggesting the significant inhibitory effect of tannic acid, which is known to inhibit ATPase.

- Tannic acid-based PTX nanoparticles inhibited cell proliferation, migration, invasion and colony-forming ability in TNBC cells.

- Microarray analysis demonstrates the induction of apoptosis with tannic acid-based PTX nanoparticles, which also induces enhanced mitotic cell cycle arrest.

The following summary can be drawn from Chapter 4:

- Combination of polyvinylpyrrolidone and tannic acid nanoparticles stabilized the nanoparticle core due to extensive hydrogen bond interaction. This gave the base core for the coating with exogenous cell membranes.

- Biomimetic nanoparticles combine cell membrane characteristics imparting biological identity to the nanoparticles. For which, we achieved cell-specific binding to the antigenreceptor on aggressive and metastatic TNBC cells due to the presence of activated neutrophil, RAW 264.7, NK-92, Mesenchymal stem cells and Jurkat cells.

- Prolonged circulation was achieved after membrane coating of the nanoparticles over uncoated nanoparticles.

- Tumor retention and biodistribution to other healthy organs was minimized in comparison to uncoated nanoparticles and drug solution.

- Adverse toxicities such as neutropenia was not observed with the membrane coated nanoparticles, suggesting the safe profile of our developed nanoparticles.

In conclusion, the hypothesis of the dissertation was to develop cell membrane coated polymeric nanoparticles that can be an effective drug delivery option for aggressive and metastatic TNBC. We herein demonstrate the development, optimization and characterization of these nanoparticles that prolongs circulation, retained at the primary tumors and reduce adverse side effects on healthy organs thus minimize immunogenicity.

\section{Limitations to the Study}

We herein demonstrate in vitro and in vivo efficacies of neutrophil membrane coated nanoparticles in comparison to either the plain drug solution or uncoated nanoparticles. However, this study was designed to evaluate the best targeting nanoparticles from the wide array of selection of endogenous cell membranes. Thus the in vivo study was primarily designed for a pilot screening of the best available membrane coated nanoparticles, where a smaller sample size of 5 mice per group was used. We only have tested the in vivo efficacies in subcutaneous xenograft mouse model by an intraperitoneal dose, yet other sophisticated in vivo efficacies and targeting studies could be explored. 


\section{Future Directions}

Although further warranting needs to be done, after estimating the sample size for in vivo studies by Power analysis, to generate the required number of animals to estimate the appropriate efficacy and targetability of neutrophil membrane coated nanoparticles in comparison to uncoated and drug solution. Further, other clinically relevant BC models such as orthotopic patient-derived xenograft and/or MMT Transgenic Mammary Tumor Mouse Models could be utilized. These mouse models are highly suitable for evaluating the effects of membrane coated nanoparticles in disease progression, metastasis, and development of drug resistance. This could warrant the potential of these biomimetic nanoparticles to target not only the primary tumor site but circulating tumor cells in the blood or at distant site. Also, this drug delivery system could be employed for evaluating other diseased conditions which has an inflamed vasculature, as in ischemia. 


\section{LIST OF REFERENCES}

1. DeSantis, C.E., et al., Breast cancer statistics, 2019. CA: a cancer journal for clinicians, 2019. 69(6): p. 438-451.

2. Malhotra, G.K., et al., Histological, molecular and functional subtypes of breast cancers. Cancer biology \& therapy, 2010. 10(10): p. 955-960.

3. $\quad$ Society;, A.C., American Cancer Society. Cancer Facts \& Figures. 2019.

4. Yersal, O. and S. Barutca, Biological subtypes of breast cancer: Prognostic and therapeutic implications. World journal of clinical oncology, 2014. 5(3): p. 412.

5. Onitilo, A.A., et al., Breast cancer subtypes based on ER/PR and Her 2 expression: comparison of clinicopathologic features and survival. Clinical medicine \& research, 2009. 7(1-2): p. 4-13.

6. Howell, S.J., S.R. Johnston, and A. Howell, The use of selective estrogen receptor modulators and selective estrogen receptor down-regulators in breast cancer. Best practice \& research Clinical endocrinology \& metabolism, 2004. 18(1): p. 47-66.

7. Wakeling, A.E., M. Dukes, and J. Bowler, A potent specific pure antiestrogen with clinical potential. Cancer research, 1991. 51(15): p. 3867-3873.

8. Smith, I.E. and M. Dowsett, Aromatase inhibitors in breast cancer. New England Journal of Medicine, 2003. 348(24): p. 2431-2442.

9. Iqbal, N. and N. Iqbal, Human epidermal growth factor receptor 2 (HER2) in cancers: overexpression and therapeutic implications. Molecular biology international, 2014. 2014.

10. Mitri, Z., T. Constantine, and R. O'Regan, The HER2 receptor in breast cancer: pathophysiology, clinical use, and new advances in therapy. Chemotherapy research and practice, 2012. 2012.

11. Slamon, D.J., et al., Use of chemotherapy plus a monoclonal antibody against HER2 for metastatic breast cancer that overexpresses HER2. New England Journal of Medicine, 2001. 344(11): p. 783-792.

12. Macias, H. and L. Hinck, Mammary gland development. Wiley Interdisciplinary Reviews: Developmental Biology, 2012. 1(4): p. 533-557.

13. Lydon, J.P., et al., Murine mammary gland carcinogenesis is critically dependent on progesterone receptor function. Cancer research, 1999. 59(17): p. 4276-4284.

14. Joshi, P.A., P.J. Goodwin, and R. Khokha, Progesterone exposure and breast cancer risk: understanding the biological roots. JAMA oncology, 2015. 1(3): p. 283-285.

15. Dawson, S., E. Provenzano, and C. Caldas, Triple negative breast cancers: clinical and prognostic implications. European journal of cancer, 2009. 45: p. 27-40.

16. Irvin Jr, W.J. and L.A. Carey, What is triple-negative breast cancer? European journal of cancer, 2008. 44(18): p. 2799-2805.

17. Collett, K., et al., A basal epithelial phenotype is more frequent in interval breast cancers compared with screen detected tumors. Cancer Epidemiology and Prevention Biomarkers, 2005. 14(5): p. 1108-1112.

18. Tischkowitz, M., et al., Use of immunohistochemical markers can refine prognosis in triple negative breast cancer. BMC cancer, 2007. 7(1): p. 134.

19. Dent, R., et al., Triple-negative breast cancer: clinical features and patterns of recurrence. Clinical cancer research, 2007. 13(15): p. 4429-4434. 
20. Mansel, R.E., O. Fodstad, and W.G. Jiang, Metastasis of breast cancer. Vol. 11. 2007: Springer.

21. Silver, D.P., et al., Efficacy of neoadjuvant Cisplatin in triple-negative breast cancer. Journal of clinical oncology, 2010. 28(7): p. 1145.

22. Le Du, F., et al., Is the future of personalized therapy in triple-negative breast cancer based on molecular subtype? Oncotarget, 2015. 6(15): p. 12890.

23. Byrski, T., et al., Response to neoadjuvant therapy with cisplatin in BRCA1-positive breast cancer patients. Breast cancer research and treatment, 2009. 115(2): p. 359-363.

24. Di Leo, A., et al., A meta-analysis of phase III trials evaluating the predictive value of HER 2 and topoisomerase II alpha in early breast cancer patients treated with CMF or anthracycline-based adjuvant therapy. 2009, AACR.

25. Bidard, F.-C., et al., p53 status and efficacy of primary anthracyclines/alkylating agentbased regimen according to breast cancer molecular classes. Annals of Oncology, 2008. 19(7): p. 1261-1265.

26. Gluz, O., et al., Triple-negative high-risk breast cancer derives particular benefit from dose intensification of adjuvant chemotherapy: results of WSG AM-01 trial. Annals of oncology, 2008. 19(5): p. 861-870.

27. Rouzier, R., et al., Breast cancer molecular subtypes respond differently to preoperative chemotherapy. Clinical cancer research, 2005. 11(16): p. 5678-5685.

28. Carey, L.A., et al., The triple negative paradox: primary tumor chemosensitivity of breast cancer subtypes. Clinical cancer research, 2007. 13(8): p. 2329-2334.

29. Liedtke, C., et al., Response to neoadjuvant therapy and long-term survival in patients with triple-negative breast cancer. Journal of clinical oncology, 2008. 26(8): p. 12751281.

30. Vera, T., et al., Relation of pre-anthracycline serum bilirubin levels to left ventricular ejection fraction after chemotherapy. The American journal of cardiology, 2015. 116(11): p. 1752-1755.

31. Rhea, I.B. and G.H. Oliveira, Illuminating anthracycline cardiotoxicity: the renaissance of evidence-based onco-cardiology. Journal of thoracic disease, 2015. 7(7): p. 1111.

32. Fossati, R., et al., Cytotoxic and hormonal treatment for metastatic breast cancer: a systematic review of published randomized trials involving 31,510 women. Journal of clinical oncology, 1998. 16(10): p. 3439-3460.

33. Sikov, W.M., et al., Frequent pathologic complete responses in aggressive stages II to III breast cancers with every-4-week carboplatin and weekly paclitaxel with or without trastuzumab: a Brown University Oncology Group Study. Journal of Clinical Oncology, 2009. 27(28): p. 4693-4700.

34. Torrisi, R., et al., Tailored preoperative treatment of locally advanced triple negative (hormone receptor negative and HER2 negative) breast cancer with epirubicin, cisplatin, and infusional fluorouracil followed by weekly paclitaxel. Cancer chemotherapy and pharmacology, 2008. 62(4): p. 667-672.

35. Leone, J., et al., Neoadjuvant platinum-based chemotherapy (CT) for triple-negative locally advanced breast cancer ( $L A B C)$ : retrospective analysis of 125 patients. Journal of Clinical Oncology, 2009. 27(15_suppl): p. 625-625.

36. Hugh, J., et al., Breast cancer subtypes and response to docetaxel in node-positive breast cancer: use of an immunohistochemical definition in the BCIRG 001 trial. Journal of clinical oncology, 2009. 27(8): p. 1168. 
37. Hayes, D.F., et al., HER2 and response to paclitaxel in node-positive breast cancer. New England Journal of Medicine, 2007. 357(15): p. 1496-1506.

38. Loesch, D., et al., A randomized, multicenter phase III trial comparing doxorubicin+ cyclophosphamide followed by paclitaxel or doxorubicin + paclitaxel followed by weekly paclitaxel as adjuvant therapy for high-risk breast cancer. Journal of Clinical Oncology, 2007. 25(18_suppl): p. 517-517.

39. Smith, I.C., et al., Neoadjuvant chemotherapy in breast cancer: significantly enhanced response with docetaxel. Journal of clinical oncology, 2002. 20(6): p. 1456-1466.

40. Lehmann, B.D., et al., Identification of human triple-negative breast cancer subtypes and preclinical models for selection of targeted therapies. The Journal of clinical investigation, 2011. 121(7): p. 2750-2767.

41. Tutt, A. and A. Ashworth, The relationship between the roles of BRCA genes in DNA repair and cancer predisposition. Trends in molecular medicine, 2002. 8(12): p. 571-576.

42. Bryant, H.E., et al., Specific killing of BRCA2-deficient tumours with inhibitors of poly (ADP-ribose) polymerase. Nature, 2005. 434(7035): p. 913.

43. Farmer, H., et al., Targeting the DNA repair defect in BRCA mutant cells as a therapeutic strategy. Nature, 2005. 434(7035): p. 917.

44. Robson, M., et al., Olaparib for metastatic breast cancer in patients with a germline BRCA mutation. New England Journal of Medicine, 2017. 377(6): p. 523-533.

45. Litton, J.K., et al., Neoadjuvant talazoparib (TALA) for operable breast cancer patients with a BRCA mutation (BRCA+). 2018, American Society of Clinical Oncology.

46. Litton, J.K., et al., Talazoparib in patients with advanced breast cancer and a germline BRCA mutation. New England Journal of Medicine, 2018. 379(8): p. 753-763.

47. Kim, S.-B., et al., Ipatasertib plus paclitaxel versus placebo plus paclitaxel as first-line therapy for metastatic triple-negative breast cancer (LOTUS): a multicentre, randomised, double-blind, placebo-controlled, phase 2 trial. The Lancet Oncology, 2017. 18(10): p. 1360-1372.

48. Dent, R., et al., Overall survival (OS) update of the double-blind placebo (PBO)controlled randomized phase 2 LOTUS trial of first-line ipatasertib (IPAT) + paclitaxel (PAC) for locally advanced/metastatic triple-negative breast cancer (mTNBC). 2018, American Society of Clinical Oncology.

49. Modi, S., et al., A phase I study of cetuximab/paclitaxel in patients with advanced-stage breast cancer. Clinical breast cancer, 2006. 7(3): p. 270-277.

50. Carey, L., et al., TBCRC 001: EGFR inhibition with cetuximab added to carboplatin in metastatic triple-negative (basal-like) breast cancer. Journal of Clinical Oncology, 2008. 26(15_suppl): p. 1009-1009.

51. Liu, T., et al., Effect of mTOR inhibition on sensitivity of triple-negative breast cancer cells to epidermal growth factor inhibition. Journal of Clinical Oncology, 2009. 27(15_suppl): p. 1055-1055.

52. Tang, D., et al., The expression and clinical significance of the androgen receptor and Ecadherin in triple-negative breast cancer. Medical oncology, 2012. 29(2): p. 526-533.

53. Cauley, J.A., et al., Elevated serum estradiol and testosterone concentrations are associated with a high risk for breast cancer. Annals of internal medicine, 1999. 130(4_Part_1): p. 270-277.

54. Rakha, E.A., et al., Prognostic markers in triple-negative breast cancer. Cancer, 2007. 109(1): p. 25-32. 
55. $\mathrm{Hu}, \mathrm{R}$. , et al., Androgen receptor expression and breast cancer survival in postmenopausal women. Clinical cancer research, 2011. 17(7): p. 1867-1874.

56. Tran, C., et al., Development of a second-generation antiandrogen for treatment of advanced prostate cancer. Science, 2009. 324(5928): p. 787-790.

57. Trials.gov, C., Feasibility Study of Adjuvant Enzalutamide for the Treatment of Early Stage AR (+) Triple Negative Breast Cancer, Identifier (NCT number): NCT02750358, . 2018.

58. Mina, A., R. Yoder, and P. Sharma, Targeting the androgen receptor in triple-negative breast cancer: current perspectives. OncoTargets and therapy, 2017. 10: p. 4675.

59. Miles, D., et al., COLET (NCT02322814): A multistage, phase 2 study evaluating the safety and efficacy of cobimetinib $(C)$ in combination with paclitaxel $(P)$ as first-line treatment for patients (pts) with metastatic triple-negative breast cancer (TNBC). 2016, American Society of Clinical Oncology.

60. Van Swearingen, A.E., et al., Combined kinase inhibitors of MEK1/2 and either PI3K or PDGFR are efficacious in intracranial triple-negative breast cancer. Neuro-oncology, 2017. 19(11): p. 1481-1493.

61. Infante, J.R., et al., A phase $1 b$ study of trametinib, an oral Mitogen-activated protein kinase kinase (MEK) inhibitor, in combination with gemcitabine in advanced solid tumours. European journal of cancer, 2013. 49(9): p. 2077-2085.

62. Tolcher, A.W., et al., Phase I study of the MEK inhibitor trametinib in combination with the AKT inhibitor afuresertib in patients with solid tumors and multiple myeloma. Cancer chemotherapy and pharmacology, 2015. 75(1): p. 183-189.

63. Tolcher, A., et al., A phase IB trial of the oral MEK inhibitor trametinib (GSK1120212) in combination with everolimus in patients with advanced solid tumors. Annals of Oncology, 2014. 26(1): p. 58-64.

64. Fry, D.W., et al., Specific inhibition of cyclin-dependent kinase 4/6 by PD 0332991 and associated antitumor activity in human tumor xenografts. Molecular cancer therapeutics, 2004. 3(11): p. 1427-1438.

65. Clark, A.S., et al., Combination paclitaxel and palbociclib: results of a phase I trial in advanced breast cancer. Clinical Cancer Research, 2019. 25(7): p. 2072-2079.

66. Cubas, R., et al., Trop2 expression contributes to tumor pathogenesis by activating the ERK MAPK pathway. Molecular cancer, 2010. 9(1): p. 253.

67. Bardia, A., et al., Efficacy and safety of anti-trop-2 antibody drug conjugate sacituzumab govitecan (IMMU-132) in heavily pretreated patients with metastatic triple-negative breast cancer. Journal of Clinical Oncology, 2017. 35(19): p. 2141.

68. Bendell, J., et al., Phase I/II study of the antibody-drug conjugate glembatumumab vedotin in patients with locally advanced or metastatic breast cancer. Journal of Clinical Oncology, 2014. 32(32): p. 3619-3625.

69. Tutt, A., et al., Oral poly (ADP-ribose) polymerase inhibitor olaparib in patients with BRCA1 or BRCA2 mutations and advanced breast cancer: a proof-of-concept trial. The Lancet, 2010. 376(9737): p. 235-244.

70. Han, H.S., et al., SGNLVA-002: Single-arm, open label phase Ib/II study of ladiratuzumab vedotin $(L V)$ in combination with pembrolizumab for first-line treatment of patients with unresectable locally advanced or metastatic triple-negative breast cancer. 2019, American Society of Clinical Oncology. 
71. Kang, T., et al., Nanoparticles coated with neutrophil membranes can effectively treat cancer metastasis. ACS nano, 2017. 11(2): p. 1397-1411.

72. Mittendorf, E.A., et al., PD-L1 expression in triple-negative breast cancer. Cancer immunology research, 2014. 2(4): p. 361-370.

73. Adams, S., et al., Phase 2 study of pembrolizumab (pembro) monotherapy for previously treated metastatic triple-negative breast cancer (mTNBC): KEYNOTE-086 cohort A. 2017, American Society of Clinical Oncology.

74. Schmid, P., et al., Atezolizumab and nab-paclitaxel in advanced triple-negative breast cancer. New England Journal of Medicine, 2018. 379(22): p. 2108-2121.

75. Greish, K., Enhanced permeability and retention (EPR) effect for anticancer nanomedicine drug targeting, in Cancer Nanotechnology. 2010, Springer. p. 25-37.

76. Grobmyer, S.R., et al., The promise of nanotechnology for solving clinical problems in breast cancer. Journal of surgical oncology, 2011. 103(4): p. 317-325.

77. Zhu, J., et al., Progress in aptamer-mediated drug delivery vehicles for cancer targeting and its implications in addressing chemotherapeutic challenges. Theranostics, 2014. 4(9): p. 931.

78. Mura, S., J. Nicolas, and P. Couvreur, Stimuli-responsive nanocarriers for drug delivery. Nature materials, 2013. 12(11): p. 991.

79. Kutty, R.V., D.T. Wei Leong, and S.-S. Feng, Nanomedicine for the treatment of triplenegative breast cancer. Nanomedicine, 2014. 9(5): p. 561-564.

80. Tan, G.-R., S.-S. Feng, and D.T. Leong, The reduction of anti-cancer drug antagonism by the spatial protection of drugs with PLA-TPGS nanoparticles. Biomaterials, 2014. 35(9): p. 3044-3051.

81. Sharma, A., N. Jain, and R. Sareen, Nanocarriers for diagnosis and targeting of breast cancer. BioMed research international, 2013. 2013.

82. Johnstone, T.C., et al., Nanoparticle encapsulation of mitaplatin and the effect thereof on in vivo properties. ACS nano, 2013. 7(7): p. 5675-5683.

83. Raiter, A., R. Yerushalmi, and B. Hardy, Pharmacological induction of cell surface GRP78 contributes to apoptosis in triple negative breast cancer cells. Oncotarget, 2014. 5(22): p. 11452.

84. Passarella, R.J., et al., Targeted nanoparticles that deliver a sustained, specific release of Paclitaxel to irradiated tumors. Cancer research, 2010. 70(11): p. 4550-4559.

85. Shi, P., et al., Elastin-based protein polymer nanoparticles carrying drug at both corona and core suppress tumor growth in vivo. Journal of controlled release, 2013. 171(3): p. 330-338.

86. Hussein, Y.R., et al., Clinical and biological relevance of enhancer of zeste homolog $2 \mathrm{in}$ triple-negative breast cancer. Human pathology, 2012. 43(10): p. 1638-1644.

87. Adams, M.L., A. Lavasanifar, and G.S. Kwon, Amphiphilic block copolymers for drug delivery. Journal of pharmaceutical sciences, 2003. 92(7): p. 1343-1355.

88. Cabral, H. and K. Kataoka, Progress of drug-loaded polymeric micelles into clinical studies. Journal of Controlled Release, 2014. 190: p. 465-476.

89. Kutty, R.V. and S.-S. Feng, Cetuximab conjugated vitamin E TPGS micelles for targeted delivery of docetaxel for treatment of triple negative breast cancers. Biomaterials, 2013. 34(38): p. 10160-10171.

90. Lainé, A.-L., et al., The in vivo performance of ferrocenyl tamoxifen lipid nanocapsules in xenografted triple negative breast cancer. Biomaterials, 2013. 34(28): p. 6949-6956. 
91. Lee, S.-M., et al., Biological evaluation of $p H$-responsive polymer-caged nanobins for breast cancer therapy. ACS nano, 2010. 4(9): p. 4971-4978.

92. Ahn, R.W., et al., A novel nanoparticulate formulation of arsenic trioxide with enhanced therapeutic efficacy in a murine model of breast cancer. Clinical cancer research, 2010. 16(14): p. 3607-3617.

93. Franchina, T., et al., Activity of pegylated liposomal doxorubicin in combination with gemcitabine in triple negative breast cancer with skin involvement: two case reports. Cancer biology \& therapy, 2012. 13(7): p. 472-476.

94. Dai, W., et al., Combined mTOR inhibitor rapamycin and doxorubicin-loaded cyclic octapeptide modified liposomes for targeting integrin $\alpha 3$ in triple-negative breast cancer. Biomaterials, 2014. 35(20): p. 5347-5358.

95. Tekedereli, I., et al., Targeted silencing of elongation factor 2 kinase suppresses growth and sensitizes tumors to doxorubicin in an orthotopic model of breast cancer. PloS one, 2012. 7(7): p. e41171.

96. Awada, A., et al., A randomized controlled phase II trial of a novel composition of paclitaxel embedded into neutral and cationic lipids targeting tumor endothelial cells in advanced triple-negative breast cancer (TNBC). Annals of oncology, 2014. 25(4): p. 824-831.

97. Bawarski, W.E., et al., Emerging nanopharmaceuticals. Nanomedicine: Nanotechnology, Biology and Medicine, 2008. 4(4): p. 273-282.

98. Wang, P., et al., Generation 4 polyamidoamine dendrimers is a novel candidate of nanocarrier for gene delivery agents in breast cancer treatment. Cancer letters, 2010. 298(1): p. 34-49.

99. Finlay, J., et al., RNA-based TWIST1 inhibition via dendrimer complex to reduce breast cancer cell metastasis. BioMed research international, 2015. 2015.

100. Raha, S., T. Paunesku, and G. Woloschak, Peptide-mediated cancer targeting of nanoconjugates. Wiley Interdisciplinary Reviews: Nanomedicine and Nanobiotechnology, 2011. 3(3): p. 269-281.

101. Mittapalli, R.K., et al., Paclitaxel-hyaluronic nanoconjugates prolong overall survival in a preclinical brain metastases of breast cancer model. Molecular cancer therapeutics, 2013. 12(11): p. 2389-2399.

102. Inoue, S., et al., Nanobiopolymer for direct targeting and inhibition of EGFR expression in triple negative breast cancer. PLoS One, 2012. 7(2): p. e31070.

103. Ljubimova, J.Y., et al., Toxicity and efficacy evaluation of multiple targeted polymalic acid conjugates for triple-negative breast cancer treatment. Journal of drug targeting, 2013. 21(10): p. 956-967.

104. Ljubimova, J.Y., et al., Nanoconjugate based on polymalic acid for tumor targeting. Chemico-biological interactions, 2008. 171(2): p. 195-203.

105. Health, N.I.o., Folic Acid-Tubulysin Conjugate EC1456 In Patients With Advanced Solid Tumors. February 7, 2019.

106. Sahoo, N.G., et al., Functionalized carbon nanomaterials as nanocarriers for loading and delivery of a poorly water-soluble anticancer drug: a comparative study. Chemical communications, 2011. 47(18): p. 5235-5237.

107. Lamprecht, C., et al., AFM imaging of functionalized carbon nanotubes on biological membranes. Nanotechnology, 2009. 20(43): p. 434001. 
108. Liu, Z., et al., Drug delivery with carbon nanotubes for in vivo cancer treatment. Cancer research, 2008. 68(16): p. 6652-6660.

109. Burke, A.R., et al., The resistance of breast cancer stem cells to conventional hyperthermia and their sensitivity to nanoparticle-mediated photothermal therapy. Biomaterials, 2012. 33(10): p. 2961-2970.

110. Khodabandehloo, H., H. Zahednasab, and A.A. Hafez, Nanocarriers usage for drug delivery in cancer therapy. Iranian journal of cancer prevention, 2016. 9(2).

111. Mfouo Tynga, I. and H. Abrahamse, Nano-Mediated Photodynamic Therapy for Cancer: Enhancement of Cancer Specificity and Therapeutic Effects. Nanomaterials, 2018. 8(11): p. 923.

112. Ozga, M., et al., A systematic review of ovarian cancer and fear of recurrence. Palliative \& supportive care, 2015. 13(6): p. 1771-1780.

113. Wang, Z., et al., Targeting miRNAs involved in cancer stem cell and EMT regulation: An emerging concept in overcoming drug resistance. Drug resistance updates, 2010. 13(4-5): p. 109-118.

114. Cho, K., et al., Therapeutic nanoparticles for drug delivery in cancer. Clinical cancer research, 2008. 14(5): p. 1310-1316.

115. Babu, A., et al., Nanoparticle-based drug delivery for therapy of lung cancer: progress and challenges. Journal of Nanomaterials, 2013. 2013: p. 14.

116. Mousa, S.A. and D.J. Bharali, Nanotechnology-based detection and targeted therapy in cancer: nano-bio paradigms and applications. Cancers, 2011. 3(3): p. 2888-2903.

117. Gupta, S., et al., Nanocarriers and nanoparticles for skin care and dermatological treatments. Indian dermatology online journal, 2013. 4(4): p. 267.

118. Kwon, G.S., Polymeric micelles for delivery of poorly water-soluble compounds. Critical Reviews $^{\text {TM }}$ in Therapeutic Drug Carrier Systems, 2003. 20(5).

119. Farokhzad, O.C., et al., Targeted nanoparticle-aptamer bioconjugates for cancer chemotherapy in vivo. Proceedings of the National Academy of Sciences, 2006. 103(16): p. 6315-6320.

120. Calvo-Aller, E., et al., First human dose escalation study in patients with metastatic malignancies to determine safety and pharmacokinetics of LY2157299, a small molecule inhibitor of the transforming growth factor-beta receptor I kinase. Journal of Clinical Oncology, 2008. 26(15_suppl): p. 14554-14554.

121. Donehower, R., et al., Phase I trial of taxol in patients with advanced cancer. 1987. 71(12): p. 1171-1177.

122. Zhang, L., et al., Human cytotoxic T-lymphocyte membrane-camouflaged nanoparticles combined with low-dose irradiation: a new approach to enhance drug targeting in gastric cancer. International journal of nanomedicine, 2017. 12: p. 2129.

123. Lang, T., et al., Ly6Chi Monocytes Delivering pH-Sensitive Micelle Loading Paclitaxel Improve Targeting Therapy of Metastatic Breast Cancer. Advanced Functional Materials, 2017. 27(26): p. 1701093.

124. Zhang, Y., et al., Macrophage-membrane-coated nanoparticles for tumor-targeted chemotherapy. Nano letters, 2018. 18(3): p. 1908-1915.

125. Zhang, Y., et al., Activated platelets-targeting micelles with controlled drug release for effective treatment of primary and metastatic triple negative breast cancer. Advanced Functional Materials, 2019. 29(13): p. 1806620. 
126. $\mathrm{Hu}, \mathrm{Q}$, et al., Relay drug delivery for amplifying targeting signal and enhancing anticancer efficacy. Advanced Materials, 2017. 29(13): p. 1605803.

127. Su, J., et al., Bioinspired Nanoparticles with NIR-Controlled Drug Release for Synergetic Chemophotothermal Therapy of Metastatic Breast Cancer. Advanced Functional Materials, 2016. 26(41): p. 7495-7506.

128. Su, J., et al., Long circulation red-blood-cell-mimetic nanoparticles with peptideenhanced tumor penetration for simultaneously inhibiting growth and lung metastasis of breast cancer. Advanced Functional Materials, 2016. 26(8): p. 1243-1252.

129. Fu, Q., et al., Programmed co-delivery of paclitaxel and doxorubicin boosted by camouflaging with erythrocyte membrane. Nanoscale, 2015. 7(9): p. 4020-4030.

130. Chen, H., et al., Lipid insertion enables targeted functionalization of paclitaxel-loaded erythrocyte membrane nanosystem by tumor-penetrating bispecific recombinant protein. International journal of nanomedicine, 2018. 13: p. 5347.

131. Gao, L., et al., Erythrocyte membrane-wrapped $\mathrm{pH}$ sensitive polymeric nanoparticles for non-small cell lung cancer therapy. Bioconjugate chemistry, 2017. 28(10): p. 2591-2598.

132. Jiang, X., et al., Erythrocyte membrane nanoparticles improve the intestinal absorption of paclitaxel. Biochemical and biophysical research communications, 2017. 488(2): p. 322-328.

133. Hao, J., et al., Neutrophils, as “Trojan horses", participate in the delivery of therapeutical PLGA nanoparticles into a tumor based on the chemotactic effect. Drug Delivery, 2020. 27(1): p. 1-14.

134. Xue, J., et al., Neutrophil-mediated anticancer drug delivery for suppression of postoperative malignant glioma recurrence. Nature nanotechnology, 2017. 12(7): p. 692.

135. Ju, C., et al., Neoadjuvant chemotherapy based on abraxane/human neutrophils cytopharmaceuticals with radiotherapy for gastric cancer. Small, 2019. 15(5): p. 1804191.

136. Tsuyuki, S., et al., Evaluation of the effect of compression therapy using surgical gloves on nanoparticle albumin-bound paclitaxel-induced peripheral neuropathy: A phase II multicenter study by the Kamigata Breast Cancer Study Group. Breast cancer research and treatment, 2016. 160(1): p. 61-67.

137. Adrada, B.E., et al., Breast implant-associated anaplastic large cell lymphoma: sensitivity, specificity, and findings of imaging studies in 44 patients. Breast cancer research and treatment, 2014. 147(1): p. 1-14.

138. Allen, T.M., Ligand-targeted therapeutics in anticancer therapy. Nature Reviews Cancer, 2002. 2(10): p. 750.

139. Albanesi, M., et al., Neutrophils mediate antibody-induced anti-tumor effects in mice. Blood, 2013: p. blood-2013-04-497446.

140. Aromal, S.A. and D. Philip, Facile one-pot synthesis of gold nanoparticles using tannic acid and its application in catalysis. Physica E: Low-dimensional Systems and Nanostructures, 2012. 44(7-8): p. 1692-1696.

141. Chu, D., J. Gao, and Z. Wang, Neutrophil-mediated delivery of therapeutic nanoparticles across blood vessel barrier for treatment of inflammation and infection. ACS nano, 2015. 9(12): p. 11800-11811.

142. Harris, L., et al., Liposome-encapsulated doxorubicin compared with conventional doxorubicin in a randomized multicenter trial as first-line therapy of metastatic breast carcinoma. Cancer, 2002.94(1): p. 25-36. 
143. Burnouf, T., et al., Platelet microparticles: detection and assessment of their paradoxical functional roles in disease and regenerative medicine. Blood reviews, 2014. 28(4): p. 155-166.

144. Simak, J. and M.P. Gelderman, Cell membrane microparticles in blood and blood products: potentially pathogenic agents and diagnostic markers. Transfusion medicine reviews, 2006. 20(1): p. 1-26.

145. Vijayan, V., S. Uthaman, and I.-K. Park, Cell membrane-camouflaged nanoparticles: A promising biomimetic strategy for cancer theragnostics. Polymers, 2018. 10(9): p. 983.

146. Yurkin, S.T. and Z. Wang, Cell membrane-derived nanoparticles: emerging clinical opportunities for targeted drug delivery. Nanomedicine, 2017. 12(16): p. 2007-2019.

147. Vader, P., X.O. Breakefield, and M.J. Wood, Extracellular vesicles: emerging targets for cancer therapy. Trends in molecular medicine, 2014. 20(7): p. 385-393.

148. Hu, C.-M.J., et al., Erythrocyte membrane-camouflaged polymeric nanoparticles as a biomimetic delivery platform. Proceedings of the National Academy of Sciences, 2011. 108(27): p. 10980-10985.

149. Parodi, A., et al., Synthetic nanoparticles functionalized with biomimetic leukocyte membranes possess cell-like functions. Nature nanotechnology, 2013. 8(1): p. 61-68.

150. Li, R., et al., Cell membrane-based nanoparticles: a new biomimetic platform for tumor diagnosis and treatment. Acta Pharmaceutica Sinica B, 2018. 8(1): p. 14-22.

151. $\mathrm{Hu}, \mathrm{C} .-\mathrm{M} . J .$, et al., Nanoparticle-detained toxins for safe and effective vaccination. Nature nanotechnology, 2013. 8(12): p. 933.

152. Laffont, B., et al., Activated platelets can deliver $m R N A$ regulatory Ago2 $\bullet$ microRNA complexes to endothelial cells via microparticles. Blood, 2013. 122(2): p. 253-261.

153. Van der Pol, E., et al., Classification, functions, and clinical relevance of extracellular vesicles. Pharmacological reviews, 2012. 64(3): p. 676-705.

154. Hamidi, M., et al., Applications of carrier erythrocytes in delivery of biopharmaceuticals. Journal of controlled release, 2007. 118(2): p. 145-160.

155. Tan, S., et al., Cell or cell membrane-based drug delivery systems. Theranostics, 2015. 5(8): p. 863.

156. Guo, Y., et al., Erythrocyte membrane-enveloped polymeric nanoparticles as nanovaccine for induction of antitumor immunity against melanoma. ACS nano, 2015. 9(7): p. 6918-6933.

157. Dehaini, D., et al., Erythrocyte-platelet hybrid membrane coating for enhanced nanoparticle functionalization. Advanced Materials, 2017. 29(16): p. 1606209.

158. Mayadas, T.N., X. Cullere, and C.A. Lowell, The multifaceted functions of neutrophils. Annual Review of Pathology: Mechanisms of Disease, 2014. 9: p. 181-218.

159. Stearns-Kurosawa, D.J., et al., The pathogenesis of sepsis. Annual review of pathology: mechanisms of disease, 2011. 6: p. 19-48.

160. Cheng, C.J., et al., A holistic approach to targeting disease with polymeric nanoparticles. Nature reviews Drug discovery, 2015. 14(4): p. 239.

161. Gao, J., D. Chu, and Z. Wang, Cell membrane-formed nanovesicles for disease-targeted delivery. Journal of controlled release, 2016. 224: p. 208-216.

162. Auffray, C., M.H. Sieweke, and F. Geissmann, Blood monocytes: development, heterogeneity, and relationship with dendritic cells. Annual review of immunology, 2009. 27: p. 669-692. 
163. Yang, J., et al., Monocyte and macrophage differentiation: circulation inflammatory monocyte as biomarker for inflammatory diseases. Biomarker research, 2014. 2(1): p. 1.

164. Jang, S.C., et al., Bioinspired exosome-mimetic nanovesicles for targeted delivery of chemotherapeutics to malignant tumors. ACS nano, 2013. 7(9): p. 7698-7710.

165. Krishnamurthy, S., et al., Monocyte cell membrane-derived nanoghosts for targeted cancer therapy. Nanoscale, 2016. 8(13): p. 6981-6985.

166. Choi, H., et al., Noninvasive imaging of radiolabeled exosome-mimetic nanovesicle using 99m Tc-HMPAO. Scientific reports, 2015. 5: p. 15636.

167. Lunavat, T.R., et al., RNAi delivery by exosome-mimetic nanovesicles-implications for targeting c-Myc in cancer. Biomaterials, 2016. 102: p. 231-238.

168. Alvarez-Erviti, L., et al., Delivery of siRNA to the mouse brain by systemic injection of targeted exosomes. Nature biotechnology, 2011. 29(4): p. 341.

169. Laoui, D., et al., Functional relationship between tumor-associated macrophages and macrophage colony-stimulating factor as contributors to cancer progression. Frontiers in immunology, 2014. 5: p. 489.

170. Hu, C.-M.J., et al., Nanoparticle biointerfacing by platelet membrane cloaking. Nature, 2015. 526(7571): p. 118.

171. Pan, V., et al., Targeted killing of metastatic cells using a platelet-inspired drug delivery system. Rsc Advances, 2015. 5(57): p. 46218-46228.

172. $\mathrm{Hu}, \mathrm{Q}$., et al., Anticancer platelet-mimicking nanovehicles. Advanced Materials, 2015. 27(44): p. 7043-7050.

173. Li, J., et al., Targeted drug delivery to circulating tumor cells via platelet membranefunctionalized particles. Biomaterials, 2016. 76: p. 52-65.

174. Rao, L., et al., Antitumor Platelet-Mimicking Magnetic Nanoparticles. Advanced Functional Materials, 2017. 27(9): p. 1604774.

175. Wani, M.C., et al., Plant antitumor agents. VI. Isolation and structure of taxol, a novel antileukemic and antitumor agent from Taxus brevifolia. Journal of the American Chemical Society, 1971. 93(9): p. 2325-2327.

176. Schiff, P.B., J. Fant, and S.B. Horwitz, Promotion of microtubule assembly in vitro by taxol. Nature, 1979. 277(5698): p. 665.

177. Walsh, V. and J. Goodman, From Taxol to Taxol $®$ : The changing identities and ownership of an anti-cancer drug. Medical anthropology, 2002. 21(3-4): p. 307-336.

178. Walsh, V. and J. Goodman, Cancer chemotherapy, biodiversity, public and private property: the case of the anti-cancer drug Taxol. Social science \& medicine, 1999. 49(9): p. 1215-1225.

179. Griffon-Etienne, G., et al., Taxane-induced apoptosis decompresses blood vessels and lowers interstitial fluid pressure in solid tumors: clinical implications. Cancer research, 1999. 59(15): p. 3776-3782.

180. Tuma, R.S., Taxol's Journey from Discovery to Use: Lessons \& Updates. Oncology Times, 2003. 25(18): p. 52-57.

181. Fu, Y., et al., Medicinal chemistry of paclitaxel and its analogues. Current medicinal chemistry, 2009. 16(30): p. 3966-3985.

182. Singla, A.K., A. Garg, and D. Aggarwal, Paclitaxel and its formulations. International journal of pharmaceutics, 2002. 235(1-2): p. 179-192. 
183. Bernabeu, E., et al., Novel Soluplus ${ }^{\circledR} — T P G S$ mixed micelles for encapsulation of paclitaxel with enhanced in vitro cytotoxicity on breast and ovarian cancer cell lines. Colloids and Surfaces B: Biointerfaces, 2016. 140: p. 403-411.

184. Walker, K., R. Long, and R. Croteau, The final acylation step in taxol biosynthesis: cloning of the taxoid C13-side-chain N-benzoyltransferase from Taxus. Proceedings of the National Academy of Sciences, 2002.99(14): p. 9166-9171.

185. Horwitz, S.B., Mechanism of action of taxol. Trends in pharmacological sciences, 1992. 13: p. 134-136.

186. Gelderblom, H., et al., Cremophor EL: the drawbacks and advantages of vehicle selection for drug formulation. European journal of cancer, 2001. 37(13): p. 1590-1598.

187. Wiernik, P.H., et al., Phase I clinical and pharmacokinetic study of taxol. Cancer research, 1987. 47(9): p. 2486-2493.

188. Sonnichsen, D.S. and M.V. Relling, Clinical pharmacokinetics of paclitaxel. Clinical pharmacokinetics, 1994. 27(4): p. 256-269.

189. Legha, S.S., D. Tenney, and I. Krakoff, Phase I study of taxol using a 5-day intermittent schedule. Journal of Clinical Oncology, 1986. 4(5): p. 762-766.

190. Scripture, C.D., W.D. Figg, and A. Sparreboom, Peripheral neuropathy induced by paclitaxel: recent insights and future perspectives. Current neuropharmacology, 2006. 4(2): p. 165-172.

191. Miele, E., et al., Albumin-bound formulation of paclitaxel (Abraxane ${ }^{\circledR}$ ABI-007) in the treatment of breast cancer. International journal of nanomedicine, 2009. 4: p. 99.

192. Zong, Y., J. Wu, and K. Shen, Nanoparticle albumin-bound paclitaxel as neoadjuvant chemotherapy of breast cancer: a systematic review and meta-analysis. Oncotarget, 2017. 8(10): p. 17360.

193. Octavia, Y., et al., Doxorubicin-induced cardiomyopathy: from molecular mechanisms to therapeutic strategies. Journal of molecular and cellular cardiology, 2012. 52(6): p. 12131225.

194. Nyman, D.W., et al., Phase I and pharmacokinetics trial of ABI-007, a novel nanoparticle formulation of paclitaxel in patients with advanced nonhematologic malignancies. Journal of clinical oncology, 2005. 23(31): p. 7785-7793.

195. Von Hoff, D.D., D. Goldstein, and M.F. Renschler, Albumin-bound paclitaxel plus gemcitabine in pancreatic cancer. The New England journal of medicine, 2014. 370(5): p. 479.

196. Rugo, H.S., et al., Randomized phase III trial of paclitaxel once per week compared with nanoparticle albumin-bound nab-paclitaxel once per week or ixabepilone with bevacizumab as first-line chemotherapy for locally recurrent or metastatic breast cancer: CALGB 40502/NCCTG N063H (Alliance). Journal of Clinical Oncology, 2015. 33(21): p. 2361.

197. Chang, A.Y., et al. Dose-escalation study of weekly 1-hour paclitaxel administration in patients with refractory cancer. in Seminars in oncology. 1997.

198. Seidman, A.D., et al., Dose-dense therapy with weekly 1-hour paclitaxel infusions in the treatment of metastatic breast cancer. Journal of Clinical Oncology, 1998. 16(10): p. 3353-3361.

199. Gradishar, W.J., et al., Phase III trial of nanoparticle albumin-bound paclitaxel compared with polyethylated castor oil-based paclitaxel in women with breast cancer. $\mathrm{J}$ Clin Oncol, 2005. 23: p. 7794-7803. 
200. Untch, M., et al., Nab-paclitaxel versus solvent-based paclitaxel in neoadjuvant chemotherapy for early breast cancer (GeparSepto-GBG 69): a randomised, phase 3 trial. The lancet oncology, 2016. 17(3): p. 345-356.

201. Wang, H., et al., Hypersensitivity reaction studies of a polyethoxylated castor oil-free, liposome-based alternative paclitaxel formulation. Molecular medicine reports, 2013. 7(3): p. 947-952.

202. NIH, Weekly and Every 3 Week Administration of Paclitaxel Liposome Injection in Metastatic Breast Cancer. 2014.

203. NIH, Weekly Paclitaxel Liposome Injection Plus Cisplatin in Preoperative Treatment of Breast Cancer. 2014.

204. Fetterly, G.J., et al., Pharmacokinetic/pharmacodynamic modeling and simulation of neutropenia during phase I development of liposome-entrapped paclitaxel. Clinical Cancer Research, 2008. 14(18): p. 5856-5863.

205. Slingerland, M., et al., Bioequivalence of Liposome-Entrapped Paclitaxel Easy-To-Use (LEP-ETU) formulation and paclitaxel in polyethoxylated castor oil: a randomized, twoperiod crossover study in patients with advanced cancer. Clinical therapeutics, 2013. 35(12): p. 1946-1954.

206. NeoPharm, NeoPharm presents the phase II data of liposome-entrapped paclitaxel (LEP) in patients with metastatic breast cancer 016.

207. Schmitt-Sody, M., et al., Neovascular targeting therapy: paclitaxel encapsulated in cationic liposomes improves antitumoral efficacy. Clinical Cancer Research, 2003. 9(6): p. 2335-2341.

208. Strieth, S., et al., Phase I/II clinical study on safety and antivascular effects of paclitaxel encapsulated in cationic liposomes for targeted therapy in advanced head and neck cancer. Head \& neck, 2014. 36(7): p. 976-984.

209. NIH, Trial of neoadjuvants EndoTAG-1 in Combination With Paclitaxel in HER2negative Breast Cancer (EndoTAG-1). 2013.

210. NIH, Carboplatin and Paclitaxel With or Without Atezolizumab Before Surgery in Treating Patients With Newly Diagnosed, Stage II-III Triple-Negative Breast Cancer. 2016.

211. Park, I.H., et al., An open-label, randomized, parallel, phase III trial evaluating the efficacy and safety of polymeric micelle-formulated paclitaxel compared to conventional cremophor EL-based paclitaxel for recurrent or metastatic HER2-negative breast cancer. Cancer research and treatment: official journal of Korean Cancer Association, 2017. 49(3): p. 569.

212. Kim, T.-Y., et al., Phase I and pharmacokinetic study of Genexol-PM, a cremophor-free, polymeric micelle-formulated paclitaxel, in patients with advanced malignancies. Clinical cancer research, 2004. 10(11): p. 3708-3716.

213. Lim, W., et al., Phase I pharmacokinetic study of a weekly liposomal paclitaxel formulation (Genexol $\mathbb{R}-P M$ ) in patients with solid tumors. Annals of oncology, 2009. 21(2): p. 382-388.

214. Jones, S., et al., Randomized phase III study of docetaxel compared with paclitaxel in metastatic breast cancer. Journal of Clinical Oncology, 2005. 23(24): p. 5542-5551.

215. Lee, K.S., et al., Multicenter phase II trial of Genexol-PM, a Cremophor-free, polymeric micelle formulation of paclitaxel, in patients with metastatic breast cancer. Breast cancer research and treatment, 2008. 108(2): p. 241-250. 
216. SORRENTO THERAPEUTICS, I., Sorrento Announces First Patient Dosed in Registration Trial to Evaluate Bioequivalence Between Cynviloq and Abraxane. March 31, 2014.

217. Pharmaceuticals, O., 2015.

218. Pharmaceutical, O., Oasmia Pharmaceutical AB, Oasmia Pharmaceutical Announces Positive Top-line Results for Paclical ${ }^{\circledR}$ From Head-to-head Comparison Study With Abraxane ${ }^{\circledR}$,. 04 Aug, 2015.

219. Ranade, A.A., et al., A multicenter phase II randomized study of Cremophor-free polymeric nanoparticle formulation of paclitaxel in women with locally advanced and/or metastatic breast cancer after failure of anthracycline. Asia-Pacific Journal of Clinical Oncology, 2013. 9(2): p. 176-181.

220. Ranade, A., et al., A novel, cremophor-free, polymeric nanoparticle paclitaxel (do/ndr/02) multicenter study in advanced/metastatic breast cancer. Journal of Clinical Oncology, 2008. 26(15_suppl): p. 1115-1115.

221. Therapeutics, C.-D., Co-D Therapeutics, Triolimus,. October, 2016.

222. Therapeutics, C.-D., Co-D Therapeutics Granted Orphan Designation for Triolimus for the Treatment of Angiosarcoma. April, 2015.

223. Hamaguchi, T., et al., NK105, a paclitaxel-incorporating micellar nanoparticle formulation, can extend in vivo antitumour activity and reduce the neurotoxicity of paclitaxel. British journal of cancer, 2005. 92(7): p. 1240.

224. Asia, P., Nippon Kayaku PhII breast cancer candidate misses endpoint. July, 2016

225. Jain, M.M., et al., Paclitaxel injection concentrate for nanodispersion versus nabpaclitaxel in women with metastatic breast cancer: a multicenter, randomized, comparative phase II/III study. Breast cancer research and treatment, 2016. 156(1): p. 125-134.

226. NIH, A Randomized, Open-Label, Comparative, Parallel-Group, Multicenter Study of SPARC1507. November, 2018.

227. NIH, Pharmacokinetic and Safety Study of SPARC1023 Alone and in Carboplatin Combination. May, 2019.

228. Terwogt, J.M.M., et al., Phase I clinical and pharmacokinetic study of PNU166945, a novel water-soluble polymer-conjugated prodrug of paclitaxel. Anti-cancer drugs, 2001. 12(4): p. 315-323.

229. Li, C., et al., Biodistribution of paclitaxel and poly (L-glutamic acid)-paclitaxel conjugate in mice with ovarian OCa-1 tumor. Cancer chemotherapy and pharmacology, 2000. 46(5): p. 416-422.

230. Boddy, A.V., et al., A phase I and pharmacokinetic study of paclitaxel poliglumex (XYOTAX), investigating both 3-weekly and 2-weekly schedules. Clinical cancer research, 2005. 11(21): p. 7834-7840.

231. NIH, Study of Xyotax (CT-2103) in Patients With Metastatic Breast Cancer. December, 2007.

232. Langer, C.J., et al., Phase III trial comparing paclitaxel poliglumex (CT-2103, PPX) in combination with carboplatin versus standard paclitaxel and carboplatin in the treatment of PS 2 patients with chemotherapy-naive advanced non-small cell lung cancer. Journal of Thoracic Oncology, 2008. 3(6): p. 623-630.

233. NIH, Oral Paclitaxel Efficacy Safety and PK in Recurrent and metAstatic Breast Cancer (OPERA). August, 2018. 
234. Constantinides, P.P., et al., Formulation development and antitumor activity of a filtersterilizable emulsion of paclitaxel. Pharmaceutical research, 2000. 17(2): p. 175-182.

235. Pharmaceuticals, S., Phase 3 Pivotal Trial of TOCOSOL ${ }^{\circledR}$ Paclitaxel Does Not Meet Primary Endpoint. September, 2007.

236. Li, F. and S.-C. Tang, Targeting metastatic breast cancer with ANG1005, a novel peptide-paclitaxel conjugate that crosses the blood-brain-barrier (BBB). 2017, Elsevier.

237. NIH, ANG1005 in Leptomeningeal Disease From Breast Cancer (ANGLeD). April, 2019.

238. Siegel, R.L., K.D. Miller, and A. Jemal, Cancer Statistics, 2017. CA Cancer J Clin, 2017. 67(1): p. 7-30.

239. Sofias, A.M., et al., The Battle of "Nano" Paclitaxel. Adv Drug Deliv Rev, 2017.

240. Donehower, R.C., The clinical development of paclitaxel: a successful collaboration of academia, industry and the National Cancer Institute. Stem Cells, 1996. 14(1): p. 25-8.

241. Montero, A.J., et al., Nab-paclitaxel in the treatment of metastatic breast cancer: $a$ comprehensive review. Expert review of clinical pharmacology, 2011. 4(3): p. 329-334.

242. Brouwer, E., et al., Measurement of fraction unbound paclitaxel in human plasma. Drug metabolism and disposition, 2000. 28(10): p. 1141-1145.

243. Di Costanzo, F., et al., Targeted delivery of albumin bound paclitaxel in the treatment of advanced breast cancer. Onco Targets Ther, 2009. 2: p. 179-188.

244. ten Tije, A.J., et al., Pharmacological effects of formulation vehicles: implications for cancer chemotherapy. Clin Pharmacokinet, 2003. 42(7): p. 665-685.

245. van Zuylen, L., J. Verweij, and A. Sparreboom, Role of formulation vehicles in taxane pharmacology. Investigational new drugs, 2001. 19(2): p. 125-141.

246. Mustacchi, G. and M. De Laurentiis, The role of taxanes in triple-negative breast cancer: literature review. Drug design, development and therapy, 2015. 9: p. 4303.

247. Oasmia. Oasmia Pharmaceutical Announces Positive Overall Survival Results from Phase III Study of Paclical/Apealea for Treatment of Ovarian Cancer. April 27, 2016; Available from: http:/oasmia.com/en/press-release/oasmia-pharmaceutical-announcespositive-overall-survival-results-phase-iii-study-paclicalapealea-treatment-ovariancancer/.

248. Koudelka, Š. and J. Turánek, Liposomal paclitaxel formulations. Journal of controlled release, 2012. 163(3): p. 322-334.

249. Havel, H., et al., Nanomedicines: from bench to bedside and beyond. The AAPS journal, 2016. 18(6): p. 1373-1378.

250. Zhang, J.A., et al., Development and characterization of a novel Cremophor ${ }^{\circledR}$ EL free liposome-based paclitaxel (LEP-ETU) formulation. European journal of pharmaceutics and biopharmaceutics, 2005. 59(1): p. 177-187.

251. Hasenstein, J.R., et al., Antitumor activity of Triolimus: a novel multidrug-loaded micelle containing Paclitaxel, Rapamycin, and 17-AAG. Molecular cancer therapeutics, 2012. 11(10): p. 2233-2242.

252. Kabanov, A.V., E.V. Batrakova, and V.Y. Alakhov, Pluronic ${ }^{\circledR}$ block copolymers for overcoming drug resistance in cancer. Advanced drug delivery reviews, 2002. 54(5): p. 759-779.

253. Suk, J.S., et al., Gene delivery to differentiated neurotypic cells with RGD and HIV Tat peptide functionalized polymeric nanoparticles. Biomaterials, 2006. 27(29): p. 51435150. 
254. Cassagneau, T., J.H. Fendler, and T.E. Mallouk, Optical and electrical characterizations of ultrathin films self-assembled from 11-Aminoundecanoic acid capped TiO2 nanoparticles and polyallylamine hydrochloride. Langmuir, 2000. 16(1): p. 241-246.

255. Kumar, R.V., et al., Preparation of amorphous magnetite nanoparticles embedded in polyvinyl alcohol using ultrasound radiation. Journal of Materials Chemistry, 2000. 10(5): p. 1125-1129.

256. Grzelczak, M., et al., Directed self-assembly of nanoparticles. ACS nano, 2010. 4(7): p. 3591-3605.

257. Lin, Y., et al., Self-directed self-assembly of nanoparticle/copolymer mixtures. Nature, 2005. 434(7029): p. 55-59.

258. Nagesh, P.K., et al., PSMA targeted docetaxel-loaded superparamagnetic iron oxide nanoparticles for prostate cancer. Colloids and Surfaces B: Biointerfaces, 2016. 144: p. 8-20.

259. Yallapu, M.M., et al., PEG-functionalized magnetic nanoparticles for drug delivery and magnetic resonance imaging applications. Pharm Res, 2010. 27(11): p. 2283-95.

260. Karthik, S., et al., A targeted, image-guided and dually locked photoresponsive drug delivery system. Journal of Materials Chemistry B, 2015. 3(5): p. 728-732.

261. Yallapu, M.M., et al., Anti-cancer activity of curcumin loaded nanoparticles in prostate cancer. Biomaterials, 2014. 35(30): p. 8635-8648.

262. Rajput, S., et al., Overcoming Akt induced therapeutic resistance in breast cancer through siRNA and thymoquinone encapsulated multilamellar gold niosomes. Molecular pharmaceutics, 2015. 12(12): p. 4214-4225.

263. Yallapu, M.M., M. Jaggi, and S.C. Chauhan, $\beta$-Cyclodextrin-curcumin self-assembly enhances curcumin delivery in prostate cancer cells. Colloids and surfaces B:

Biointerfaces, 2010. 79(1): p. 113-125.

264. Yallapu, M.M., et al., Fabrication of curcumin encapsulated PLGA nanoparticles for improved therapeutic effects in metastatic cancer cells. Journal of colloid and interface science, 2010. 351(1): p. 19-29.

265. Puvvada, N., et al., Exploring the fluorescence switching phenomenon of curcumin encapsulated niosomes: in vitro real time monitoring of curcumin release to cancer cells. RSC Advances, 2013. 3(8): p. 2553-2557.

266. Jordan, M.A. and L. Wilson, Microtubules and actin filaments: dynamic targets for cancer chemotherapy. Curr Opin Cell Biol, 1998. 10(1): p. 123-30.

267. Kwon, G., et al., Enhanced tumor accumulation and prolonged circulation times of micelle-forming poly (ethylene oxide-aspartate) block copolymer-adriamycin conjugates. Journal of Controlled Release, 1994. 29(1-2): p. 17-23.

268. Klok, H.A. and S. Lecommandoux, Supramolecular Materials via Block Copolymer SelfAssembly. Adv Mater, 2001. 13(16): p. 1217-1229.

269. Qin, S.Y., et al., Drug self-delivery systems for cancer therapy. Biomaterials, 2017. 112: p. 234-247.

270. Chowdhury, P., et al., Pluronic Nanotechnology for Overcoming Drug Resistance, in Bioactivity of Engineered Nanoparticles. 2017, Springer. p. 207-237.

271. Cho, H.-J., et al., Polyethylene glycol-conjugated hyaluronic acid-ceramide selfassembled nanoparticles for targeted delivery of doxorubicin. Biomaterials, 2012. 33(4): p. 1190-1200. 
272. Yadav, A.K., et al., Development and characterization of hyaluronic acid decorated PLGA nanoparticles for delivery of 5-fluorouracil. Drug delivery, 2010. 17(8): p. 561572.

273. Cui, W., J. Li, and G. Decher, Self-Assembled Smart Nanocarriers for Targeted Drug Delivery. Adv Mater, 2016. 28(6): p. 1302-11.

274. Shi, J., et al., Self-assembled targeted nanoparticles: evolution of technologies and bench to bedside translation. Acc Chem Res, 2011. 44(10): p. 1123-34.

275. Huang, J., et al., Biodegradable self-assembled nanoparticles of poly (d, l-lactide-coglycolide)/hyaluronic acid block copolymers for target delivery of docetaxel to breast cancer. Biomaterials, 2014. 35(1): p. 550-566.

276. Farokhzad, O.C. and R. Langer, Impact of nanotechnology on drug delivery. ACS nano, 2009. 3(1): p. 16-20.

277. Sharma, D., et al., Novel Taxol formulation: polyvinylpyrrolidone nanoparticleencapsulated Taxol for drug delivery in cancer therapy. Oncol Res, 1996. 8(7-8): p. 2816.

278. Gaucher, G., et al., Poly(N-vinyl-pyrrolidone)-block-poly(D,L-lactide) as polymeric emulsifier for the preparation of biodegradable nanoparticles. J Pharm Sci, 2007. 96(7): p. $1763-75$.

279. Xu, H., et al., An efficient Trojan delivery of tetrandrine by poly(N-vinylpyrrolidone)block-poly(epsilon-caprolactone) (PVP-b-PCL) nanoparticles shows enhanced apoptotic induction of lung cancer cells and inhibition of its migration and invasion. Int J Nanomedicine, 2014. 9: p. 231-42.

280. Zhu, Z., et al., Paclitaxel-loaded poly(N-vinylpyrrolidone)-b-poly(epsilon-caprolactone) nanoparticles: preparation and antitumor activity in vivo. J Control Release, 2010. 142(3): p. 438-46.

281. Liu, X., et al., Pharmacokinetics, tissue distribution and anti-tumour efficacy of paclitaxel delivered by polyvinylpyrrolidone solid dispersion. J Pharm Pharmacol, 2012. 64(6): p. 775-82.

282. Sawicki, E., et al., Pharmaceutical development of an oral tablet formulation containing a spray dried amorphous solid dispersion of docetaxel or paclitaxel. Int J Pharm, 2016. 511(2): p. 765-73.

283. Rowinsky, E.K., L.A. Cazenave, and R.C. Donehower, Taxol: a novel investigational antimicrotubule agent. J Natl Cancer Inst, 1990. 82(15): p. 1247-59.

284. Jordan, M.A. and L. Wilson, Microtubules as a target for anticancer drugs. Nature Reviews Cancer, 2004. 4(4): p. 253-265.

285. Antonsson, B. and J.-C. Martinou, The Bcl-2 protein family. Experimental cell research, 2000. 256(1): p. 50-57.

286. Guo, D.-D., et al., Enhanced anticancer effect of conjugated linoleic acid by conjugation with Pluronic F127 on MCF-7 breast cancer cells. Cancer letters, 2007. 254(2): p. 244254.

287. Kamada, H., et al., Antitumor activity of tumor necrosis factor- $\alpha$ conjugated with polyvinylpyrrolidone on solid tumors in mice. Cancer research, 2000. 60(22): p. 64166420.

288. McGarrigle, D., et al., Role of tyrosine kinase Csk in G protein-coupled receptor-and receptor tyrosine kinase-induced fibroblast cell migration. Journal of Biological Chemistry, 2006. 281(15): p. 10583-10588. 
289. Wu, J., et al., Response and prognosis of taxanes and anthracyclines neoadjuvant chemotherapy in patients with triple-negative breast cancer. Journal of cancer research and clinical oncology, 2011. 137(10): p. 1505.

290. Sakuma, K., et al., Pathological tumor response to neoadjuvant chemotherapy using anthracycline and taxanes in patients with triple-negative breast cancer. Experimental and therapeutic medicine, 2011. 2(2): p. 257-264.

291. Nguyen, H.N., et al., Curcumin as fluorescent probe for directly monitoring in vitro uptake of curcumin combined paclitaxel loaded PLA-TPGS nanoparticles. Advances in Natural Sciences: Nanoscience and Nanotechnology, 2016. 7(2): p. 025001.

292. Kris-Etherton, P.M., et al., Bioactive compounds in foods: their role in the prevention of cardiovascular disease and cancer. The American journal of medicine, 2002. 113(9): p. 71-88.

293. Parzel, C., Tannic Acid Crosslinked Collagens and Potential for Breast Tissue Engineering. 2006.

294. Jackson, J.K. and K. Letchford, The Effective Solubilization of Hydrophobic Drugs Using Epigallocatechin Gallate or Tannic Acid-Based Formulations. J Pharm Sci, 2016. 105(10): p. 3143-3152.

295. O'Shaughnessy, J., Extending survival with chemotherapy in metastatic breast cancer. The oncologist, 2005. 10(Supplement 3): p. 20-29.

296. Hennenfent, K. and R. Govindan, Novel formulations of taxanes: a review. Old wine in a new bottle? Annals of oncology, 2005. 17(5): p. 735-749.

297. Heldin, C.-H., et al., High interstitial fluid pressure-an obstacle in cancer therapy. Nature Reviews Cancer, 2004. 4(10): p. 806.

298. Yallapu, M.M., et al., Implications of protein corona on physico-chemical and biological properties of magnetic nanoparticles. Biomaterials, 2015. 46: p. 1-12.

299. Kumar, B.P., et al., Sequential release of drugs from hollow manganese ferrite nanocarriers for breast cancer therapy. Journal of Materials Chemistry B, 2015. 3(1): p. 90-101.

300. Chowdhury, P., et al., Development of polyvinylpyrrolidone/paclitaxel self-assemblies for breast cancer. Acta pharmaceutica sinica B, 2018. 8(4): p. 602-614. https://doi.org/10.1016/j.apsb.2017.10.004

301. MacCallum, D.K., et al., Bovine corneal endothelium in vitro: elaboration and organization of a basement membrane. Experimental cell research, 1982. 139(1): p. 1-13.

302. Hafeez, B.B., et al., Ormeloxifene Suppresses Prostate Tumor Growth and Metastatic Phenotypes via Inhibition of Oncogenic beta-catenin Signaling and EMT Progression. Mol Cancer Ther, 2017. 16(10): p. 2267-2280.

303. Nadakavukaren, K.K., J.J. Nadakavukaren, and L.B. Chen, Increased rhodamine 123 uptake by carcinoma cells. Cancer research, 1985. 45(12 Part 1): p. 6093-6099.

304. Jaggi, M., et al., E-cadherin phosphorylation by protein kinase D1/protein kinase C $\mu$ is associated with altered cellular aggregation and motility in prostate cancer. Cancer research, 2005. 65(2): p. 483-492.

305. Bharti, R., et al., Somatostatin receptor targeted liposomes with Diacerein inhibit IL-6 for breast cancer therapy. Cancer letters, 2017. 388: p. 292-302.

306. Nagesh, P.K., et al., Tannic Acid Induces Endoplasmic Reticulum Stress-Mediated Apoptosis in Prostate Cancer. Cancers, 2018. 10(3): p. 68. 
307. Khan, S., et al., Nanoparticle formulation of ormeloxifene for pancreatic cancer. Biomaterials, 2015. 53: p. 731-743.

308. Kitagawa, S., et al., Inhibition of P-glycoprotein function by tannic acid and pentagalloylglucose. Journal of pharmacy and pharmacology, 2007. 59(7): p. 965-969.

309. Litman, T., et al., Competitive, non-competitive and cooperative interactions between substrates of P-glycoprotein as measured by its ATPase activity. Biochimica et Biophysica Acta (BBA)-Molecular Basis of Disease, 1997. 1361(2): p. 169-176.

310. Shen, M., T. Hang Chan, and Q. Ping Dou, Targeting tumor ubiquitin-proteasome pathway with polyphenols for chemosensitization. Anti-Cancer Agents in Medicinal Chemistry (Formerly Current Medicinal Chemistry-Anti-Cancer Agents), 2012. 12(8): p. 891-901.

311. Bollag, D.M., et al., Epothilones, a new class of microtubule-stabilizing agents with a taxol-like mechanism of action. Cancer research, 1995. 55(11): p. 2325-2333.

312. Su, W.-P., et al., PLGA nanoparticles codeliver paclitaxel and Stat3 siRNA to overcome cellular resistance in lung cancer cells. International journal of nanomedicine, 2012. 7: p. 4269.

313. Geldenhuys, W., et al., Brain-targeted delivery of paclitaxel using glutathione-coated nanoparticles for brain cancers. Journal of drug targeting, 2011. 19(9): p. 837-845.

314. Galati, G. and P.J. O'brien, Potential toxicity of flavonoids and other dietary phenolics: significance for their chemopreventive and anticancer properties. Free Radical Biology and Medicine, 2004. 37(3): p. 287-303.

315. Tikoo, K., M.S. Sane, and C. Gupta, Tannic acid ameliorates doxorubicin-induced cardiotoxicity and potentiates its anti-cancer activity: potential role of tannins in cancer chemotherapy. Toxicology and applied pharmacology, 2011. 251(3): p. 191-200.

316. Huo, S., et al., Superior penetration and retention behavior of $50 \mathrm{~nm}$ gold nanoparticles in tumors. Cancer research, 2012.

317. Fröhlich, E., The role of surface charge in cellular uptake and cytotoxicity of medical nanoparticles. International journal of nanomedicine, 2012. 7: p. 5577.

318. Varanka, Z., et al., Biochemical and morphological changes in carp (Cyprinus carpio L.) liver following exposure to copper sulfate and tannic acid. Comparative Biochemistry and Physiology Part C: Toxicology \& Pharmacology, 2001. 128(3): p. 467-477.

319. Zhao, X., et al., Antioxidant properties of two gallotannins isolated from the leaves of Pistacia weinmannifolia. Biochimica et Biophysica Acta (BBA)-General Subjects, 2005. 1725(1): p. 103-110.

320. Formentini, L., et al., Mono-galloyl glucose derivatives are potent poly (ADP-ribose) glycohydrolase (PARG) inhibitors and partially reduce PARP-1-dependent cell death. British journal of pharmacology, 2008. 155(8): p. 1235-1249.

321. Zasadil, L.M., et al., Cytotoxicity of paclitaxel in breast cancer is due to chromosome missegregation on multipolar spindles. Science translational medicine, 2014. 6(229): p. 229ra43-229ra43.

322. Sankala, H.M., et al., Involvement of sphingosine kinase 2 in p53-independent induction of 21 by the chemotherapeutic drug doxorubicin. Cancer research, 2007. 67(21): p. 10466-10474.

323. Holohan, C., et al., Cancer drug resistance: an evolving paradigm. Nature Reviews Cancer, 2013. 13(10): p. 714. 
324. Chavanpatil, M.D., Y. Patil, and J. Panyam, Susceptibility of nanoparticle-encapsulated paclitaxel to P-glycoprotein-mediated drug efflux. International journal of pharmaceutics, 2006. 320(1-2): p. 150-156.

325. Siegel, R.L., K.D. Miller, and A.J.C.a.c.j.f.c. Jemal, Cancer statistics, 2015. 2015. 65(1): p. 5-29.

326. DeSantis, C., et al., Breast cancer statistics, 2013. CA: a cancer journal for clinicians, 2014. 64(1): p. 52-62.

327. Bernard-Marty, C., F. Cardoso, and M.J. Piccart, Facts and controversies in systemic treatment of metastatic breast cancer. The Oncologist, 2004. 9(6): p. 617-632.

328. Zhao, M., et al., Use of liposomal doxorubicin for adjuvant chemotherapy of breast cancer in clinical practice. 2017. 18(1): p. 15-26.

329. Lobbezoo, D., et al., Prognosis of metastatic breast cancer: are there differences between patients with de novo and recurrent metastatic breast cancer? 2015. 112(9): p. 1445.

330. Rowinsky, E.K., et al., Neurotoxicity of taxol. 1993(15): p. 107-115.

331. Wolf, S., et al., Chemotherapy-induced peripheral neuropathy: prevention and treatment strategies. 2008. 44(11): p. 1507-1515.

332. Kunitoh, H., et al., Neuromuscular toxicities of paclitaxel $210 \mathrm{mg} \mathrm{m}$-2 by 3-hour infusion. 1998. 77(10): p. 1686.

333. Matsumura, Y. and H.J.C.r. Maeda, A new concept for macromolecular therapeutics in cancer chemotherapy: mechanism of tumoritropic accumulation of proteins and the antitumor agent smancs. 1986. 46(12 Part 1): p. 6387-6392.

334. Safra, T., et al., Pegylated liposomal doxorubicin (doxil): reduced clinical cardiotoxicity in patients reaching or exceeding cumulative doses of $500 \mathrm{mg} / \mathrm{m} 2$. Annals of Oncology, 2000. 11(8): p. 1029-1033.

335. García, I., et al., Glycans as biofunctional ligands for gold nanorods: stability and targeting in protein-rich media. Journal of the American Chemical Society, 2015. 137(10): p. 3686-3692.

336. Yoo, J.-W., E. Chambers, and S. Mitragotri, Factors that control the circulation time of nanoparticles in blood: challenges, solutions and future prospects. Current pharmaceutical design, 2010. 16(21): p. 2298-2307.

337. Moghimi, S.M., A.C. Hunter, and J.C. Murray, Nanomedicine: current status and future prospects. The FASEB journal, 2005. 19(3): p. 311-330.

338. Pirollo, K.F. and E.H. Chang, Does a targeting ligand influence nanoparticle tumor localization or uptake? Trends in biotechnology, 2008. 26(10): p. 552-558.

339. F. Danhier, O.F.a.V.P., To exploit the tumor microenvironment: passive and active tumor targeting of nanocarriers for anti-cancer drug delivery. Journal of controlled release, 2010. 148: p. 135-146.

340. Torchilin, V.P., [PDF] umass.edu Recent advances with liposomes as pharmaceutical carriers. Nat. Rev. Drug Discovery 2005. 4.

341. Yoo, J.-W., et al., Bio-inspired, bioengineered and biomimetic drug delivery carriers. Nature reviews Drug discovery, 2011. 10(7): p. 521.

342. Gao, J., S. Wang, and Z. Wang, High yield, scalable and remotely drug-loaded neutrophil-derived extracellular vesicles (EVs) for anti-inflammation therapy. Biomaterials, 2017. 135: p. 62-73. 
343. Naus, P.J., et al., Tannic acid synergizes the cytotoxicity of chemotherapeutic drugs in human cholangiocarcinoma by modulating drug efflux pathways. Journal of hepatology, 2007. 46(2): p. 222-229.

344. Chowdhury, P., et al., Tannic acid-inspired paclitaxel nanoparticles for enhanced anticancer effects in breast cancer cells. Journal of colloid and interface science, 2019. 535: p. 133-148. https://doi.org/10.1016/j.jcis.2018.09.072

345. McCloy, R.A., et al., Partial inhibition of Cdk1 in G2 phase overrides the SAC and decouples mitotic events. Cell cycle, 2014. 13(9): p. 1400-1412.

346. Chauhan, S.C., et al., Expression and functions of transmembrane mucin MUC13 in ovarian cancer. Cancer research, 2009. 69(3): p. 765-774.

347. Gbassi, G., et al., Whey proteins analysis in aqueous medium and in artificial gastric and intestinal fluids. International Journal of Biological and Chemical Sciences, 2012. 6(4): p. 1828-1837.

348. Ha, P.T., et al., Targeted drug delivery nanosystems based on copolymer poly (lactide)tocopheryl polyethylene glycol succinate for cancer treatment. Advances in Natural Sciences: Nanoscience and Nanotechnology, 2016. 7(1): p. 015001.

349. Rajar, K., et al., Tannic acid assisted copper oxide nanoglobules for sensitive electrochemical detection of bisphenol A. International Journal of Food Properties, 2017. 20(6): p. 1359-1367.

350. Dhumale, V.A., et al., Reversible aggregation control of polyvinylpyrrolidone capped gold nanoparticles as a function of pH. Materials Express, 2012. 2(4): p. 311-318.

351. Denizli, M., et al., Chitosan nanoparticles for miRNA delivery, in RNA Nanostructures. 2017, Springer. p. 219-230.

352. Ye, J., et al., Improved safety and efficacy of a lipid emulsion loaded with a paclitaxelcholesterol complex for the treatment of breast tumors. Oncology reports, 2016. 36(1): p. 399-409.

353. Venook, A.P., et al., Phase I and pharmacokinetic trial of paclitaxel in patients with hepatic dysfunction: Cancer and Leukemia Group B 9264. Journal of clinical oncology, 1998. 16(5): p. 1811-1819.

354. Grigorian, A. and C.B. O'Brien, Hepatotoxicity secondary to chemotherapy. Journal of clinical and translational hepatology, 2014. 2(2): p. 95.

355. Nascimento, T.G.d., et al., Neutropenia: occurrence and management in women with breast cancer receiving chemotherapy. Revista latino-americana de enfermagem, 2014. 22(2): p. 301-308.

356. Blanco, E., H. Shen, and M.J.N.b. Ferrari, Principles of nanoparticle design for overcoming biological barriers to drug delivery. 2015. 33(9): p. 941.

357. Li, R., He, Y., Zhang, S., Qin, J., \& Wang, J., Cell membrane-based nanoparticles: a new biomimetic platform for tumor diagnosis and treatment. Acta Pharmaceutica Sinica B, 2018. 8(1): p. 14-22.

358. Zhu, D.-M., et al., Erythrocyte membrane-coated gold nanocages for targeted photothermal and chemical cancer therapy. Nanotechnology, 2018. 29(8): p. 084002.

359. Pitchaimani, A., et al., Biomimetic Natural Killer Membrane Camouflaged Polymeric Nanoparticle for Targeted Bioimaging. Advanced Functional Materials, 2019. 29(4): p. 1806817.

360. Gao, C., et al., Stem cell membrane-coated nanogels for highly efficient in vivo tumor targeted drug delivery. Small, 2016. 12(30): p. 4056-4062. 
361. Dinauer, N., et al., Selective targeting of antibody-conjugated nanoparticles to leukemic cells and primary T-lymphocytes. Biomaterials, 2005. 26(29): p. 5898-5906.

362. Parodi, A., et al., Synthetic nanoparticles functionalized with biomimetic leukocyte membranes possess cell-like functions. Nature nanotechnology, 2013. 8(1): p. 61.

363. Le, Z., et al., Hydrogen-bonded tannic acid-based anticancer nanoparticle for enhancement of oral chemotherapy. ACS applied materials \& interfaces, 2018. 10(49): p. $42186-42197$.

364. Szakács, G., et al., Targeting multidrug resistance in cancer. Nature reviews Drug discovery, 2006. 5(3): p. 219.

365. Bao, L., et al., Increased expression of P-glycoprotein and doxorubicin chemoresistance of metastatic breast cancer is regulated by miR-298. The American journal of pathology, 2012. 180(6): p. 2490-2503.

366. Lhommé, C., et al., Phase III study of valspodar (PSC 833) combined with paclitaxel and carboplatin compared with paclitaxel and carboplatin alone in patients with stage IV or suboptimally debulked stage III epithelial ovarian cancer or primary peritoneal cancer. Journal of Clinical Oncology, 2008. 26(16): p. 2674-2682.

367. Chu, D., et al., Neutrophil-Based Drug Delivery Systems. Advanced Materials, 2018. 30(22): p. 1706245.

368. Cao, X., et al., Neutrophil-mimicking therapeutic nanoparticles for targeted chemotherapy of pancreatic carcinoma. Acta Pharmaceutica Sinica B, 2019. 9(3): p. 575589.

369. Gearing, A.J., et al., Soluble forms of vascular adhesion molecules, E-selectin, ICAM-1, and VCAM-1: pathological significance. Annals of the New York Academy of Sciences, 1992. 667(1): p. 324-331.

370. Gearing, A.J. and W. Newman, Circulating adhesion molecules in disease. Immunology today, 1993. 14(10): p. 506-512.

371. El Maksoud, N.A., et al., Potential Value of ICAM-1 as a Biomarker for Detection of Progression and Prognosis in Breast Carcinoma. American Journal of Biochemistry and Molecular Biology, 7: 91, 2017. 95.

372. Costello, R.T., et al., Defective expression and function of natural killer cell-triggering receptors in patients with acute myeloid leukemia. Blood, 2002. 99(10): p. 3661-3667.

373. Mamessier, E., et al., Human breast cancer cells enhance self tolerance by promoting evasion from NK cell antitumor immunity. The Journal of clinical investigation, 2011. 121(9): p. 3609-3622.

374. Pasero, C., et al., Highly effective NK cells are associated with good prognosis in patients with metastatic prostate cancer. Oncotarget, 2015. 6(16): p. 14360.

375. Gillgrass, A. and A. Ashkar, Stimulating natural killer cells to protect against cancer: recent developments. Expert review of clinical immunology, 2011. 7(3): p. 367-382.

376. Barsoum, I.B., et al., Hypoxia induces escape from innate immunity in cancer cells via increased expression of ADAM10: role of nitric oxide. Cancer research, 2011. 71(24): $\mathrm{p}$. 7433-7441.

377. Shenouda, M.M., et al., Ex vivo expanded natural killer cells from breast cancer patients and healthy donors are highly cytotoxic against breast cancer cell lines and patientderived tumours. Breast Cancer Research, 2017. 19(1): p. 76. 
378. Alshareeda, A.T., et al., The effect of human placental chorionic villi derived mesenchymal stem cell on triple-negative breast cancer hallmarks. PloS one, 2018. 13(11): p. e0207593.

379. Palomba, R., et al., Biomimetic carriers mimicking leukocyte plasma membrane to increase tumor vasculature permeability. Scientific reports, 2016. 6: p. 34422.

380. Cao, H., et al., Liposomes coated with isolated macrophage membrane can target lung metastasis of breast cancer. ACS nano, 2016. 10(8): p. 7738-7748.

381. Luk, B.T., et al., Interfacial interactions between natural RBC membranes and synthetic polymeric nanoparticles. Nanoscale, 2014. 6(5): p. 2730-2737.

382. Möller, M. and A. Denicola, Protein tryptophan accessibility studied by fluorescence quenching. Biochemistry and Molecular Biology Education, 2002. 30(3): p. 175-178.

383. Hilbig, A., et al., Preliminary results of a phase I/II study in patients with pancreatic carcinoma, malignant melanoma, or colorectal carcinoma using systemic iv administration of AP 12009. Journal of Clinical Oncology, 2008. 26(15_suppl): p. 46214621.

384. Peiris, P.M., et al., Imaging metastasis using an integrin-targeting chain-shaped nanoparticle. ACS nano, 2012. 6(10): p. 8783-8795.

385. Duan, X., et al., Smart $p H$-sensitive and temporal-controlled polymeric micelles for effective combination therapy of doxorubicin and disulfiram. ACS nano, 2013. 7(7): p. 5858-5869.

386. Hoffman, R.M., Orthotopic metastatic mouse models for anticancer drug discovery and evaluation: a bridge to the clinic. Investigational new drugs, 1999. 17(4): p. 343-360.

387. Borges, S., et al., Effective Targeting of Estrogen Receptor-Negative Breast Cancers with the Protein Kinase D Inhibitor CRT0066101. Molecular cancer therapeutics, 2015. 14(6): p. 1306-1316. 


\section{VITA}

Pallabita Chowdhury was born in Kolkata, India in the year 1990. She obtained Bachelor of Pharmacy degree on December 2012 from Jadavpur University, Kolkata, India and Master of Science in Industrial Pharmacy on August 2015 from University of Toledo, Ohio, US. She was matriculated in Pharmaceutics in Pharmaceutical Sciences for doctoral studies at University of Tennessee Health Science Center, Memphis, TN, USA in August 2015. She was awarded the Alma and Hall Reagan Endowment Fellowship in September 2019 from UTHSC. She anticipates completion of the requirements for doctorate degree by May 2020. 